\title{
On the Mechanisms of Hydrogen Implantation Induced Silicon Surface Layer Cleavage
}

\author{
Dissertation \\ zur \\ Erlangung des Doktorgrades der Naturwissenschaften \\ (Dr. rer. nat.) \\ dem \\ Fachbereich Chemie \\ der Philipps-Universität Marburg \\ vorgelegt von
}

\section{Tobias Höchbauer \\ aus Mallersdorf}

Marburg/Lahn, November 2001 



\section{Vom Fachbereich Chemie \\ der Philipps-Universität Marburg als Dissertation am 17.12.2001 angenommen. \\ Erstgutachter: Prof. Dr. W. Ensinger \\ Zweitgutachter: Prof. Dr. G.K. Wolf (Universität Heidelberg)}

Tag der mündlichen Prüfung: 18.12.2001 



\section{The English version of this summary appears on page 185}

\section{Zusammenfassung}

Der sogenannte "Ion-Cut", ein Schichtabspaltungsprozess mittels WasserstoffIonenimplantation und darauffolgender Probenerwärmung ist eine effektive, vielseitige und ökonomische Methode, um Siliziumschichten mit einer Dicke von nur einigen Nanometern von einem Siliziumsubstrat auf andere Materialien zu transferieren. Somit ermöglicht der Ion-Cut die Produktion von Siliziumschichen auf isolierenden Materalien ("silicon-on-insulator" (SOI)). Die Abspaltung ist durch das Zusammenwachsen von vergrabenen hochkomprimierten Wasserstoffgasblasen verursacht, die während der Probeerhitzung entstehen. Obwohl das Phänomen des Ion-Cuts weit verbreitete Anwendung findet, sind die fundamentalen Mechanismen dieses Abspaltungsprozesses nach wie vor nicht verstanden, was eine weitere Optimierung des Ion-Cut Prozesses verhindert.

Gegenstand dieser Arbeit war die Untersuchung und Aufdeckung der physikalischen Mechanismen des Ion-Cut-Prozesses in Wasserstoff implantiertem Silizium. Dies erfolgte, indem die Entwicklung der Siliziumkristallschäden, verursacht durch die Wasserstoffimplantation, untersucht wurde und die erzielten Ergebnisse in Korrelation gebracht wurden zu den experimentell gewonnenen Daten über die Wasserstoff-Tiefenverteilung, der Mikrostruktur der Implantationszone und der Position des Bruchs in der Implantationszone. Die Untersuchung des Ion-Cuts zeigte auf, dass der Abspaltungsprozess nicht von der Wasserstoff-Tiefenverteilung kontrolliert wird, sondern von den durch den Ionenbeschuss erzeugten Kristallgitterschäden und deren Einfluss auf das lokale Spannungsfeld und die Bruchfestigkeit in der Implantationszone.

Detaillierte Studien an mit moderaten Wasserstoffdosen implantierten Proben zeigten auf, dass sich der Abspaltungsprozess in einer Tiefe ereignet, in der die Strahlenschäden ihr Maximum erreichen. Diese Erkenntnis steht im Widerspruch zur allgemeinen Annahme, dass sich die Abspaltung in der Tiefe der maximalen Wasserstoffkonzentration ereignet. Die Ergebnisse wurden durch eine Kombination von Ionenstrahlanalyse-Methoden, Sekundär-Ionen-Massenspektroskopie, ex-situ Messungen des internen Spannungsaufbaus in der Implantationszone und Elektronenmikroskopie gewonnen. Diese erzielten Ergebnisse wurden damit begründet, dass die durch den 
Protonenbeschuss entstandenen Kristallschäden und deren Einfluss auf das lokale Spannungsfeld die Dehnung des Kristallgitters senkrecht zur Siliziumoberfläche zur Folge hat, was sich wiederum auf die Tiefe des Ion-Cuts auswirkt: Es stellte sich heraus, dass die Dehnung des Kristallgitters die Einbettung von Wasserstoffatomen in bindungszentrierte Zwischengitterplätze zwischen je zwei Siliziumgitteratomen beschleunigt, was die Ausbildung von (100)-orientierten Wasserstoffplättchen parallel zur Probenoberfläche bewirkt. Diese Wasserstoffplättchen sind die Nukleonen der vergrabenen Wasserstoffgasblasen, die für die Abspaltung verantwortlich sind.

Die oben erwähnten Hypothesen wurden durch Untersuchungen des Ion-Cuts an Proben mit grösseren Wasserstoffimplantationsdosen bestätigt. Die Erforschung des Abspaltungsprozesses an Proben mit verschiedenen Implantationsdosen zeigte auf, dass sich die Abspaltung in zwei verschiedenen Tiefen in der Implantationszone ereignet: entweder in einer Tiefe in der die Kristallschäden ihr Maximum erreichen, oder im hinteren Bereich der etwas tiefer gelegenen Wasserstoffverteilung. Diese Arbeit deckt zum ersten Mal auf, welche Auswirkung die durch die Wasserstoffimplantation erzeugten Strahlenschäden auf die Bruchfestigkeit des Silizium haben, und, wie die sich ändernde Bruchfestigkeit die Abspaltungstiefe beeinflusst. Die in Korrelation zueinander gebrachten Daten führen zu dem Schluss, dass die Verlagerung des Ion-Cuts hinzu zu grösseren Tiefen mit ansteigender Implantationsdosis sich aufgrund der verändernden Bruchfestigkeit des Materials ereignet. Dieses grundlegende Verständnis davon, wie sich die Abspaltungsmechanismen mit steigender Wasserstoffimplantationsdosis verändern, zeigte die entscheidenden Beziehungen zwischen Bruchfestigkeit, Spannung, Punktfehlern und ausgedehnten Gitterfehlern auf, die sich als verantwortlich für den IonCut-Prozess in Silizium ausmachen lassen.

Genauere Informationen über die Kinetik der Wasserstoffkomplexbildung in Silizium und deren Einfluss auf die Kristallschäden und fortlaufend die Wasserstoffplättchenbildung und -orientierung wurden von protonenimplantierten Siliziumproben gewonnen, die mit verschiedenen Temperaturen zwischen $200{ }^{\circ} \mathrm{C}$ and $530{ }^{\circ} \mathrm{C}$ erhitzt wurden. Die Analyse zeigte auf, dass die Probenerhitzung die Bildung von $\mathrm{H}_{2}$-Molekülen im Siliziumgitter hervorruft, und diese zu einem erheblichen Anstieg von Siliziumatomen führen, die von ihren Gitterplätzen verdrängt wurden. Diese Wasserstoffmoleküle befinden sich nicht in den $\mathrm{H}_{2}$-Gasblasen, jedoch beeinflusst die von ihnen erzeugte Kristallschädigung und das daraus resultierende Spannungs- und Dehnungsfeld im Material die Bildung von Wasserstoffplättchen, sowie deren Dichteund Tiefenverteilung. Diese Ergebnisse wurden anhand der Untersuchungen von Veränderungen der Kristallschäden- und Wasserstofftiefenverteilung erzielt, bewirkt 
durch die Probenerhitzung bei verschiedenen Temperaturen. Die Experimente sind an zwei Siliziumsubstraten mit unterschiedlichen Leitungstypen ausgeführt worden. Pleitende Siliziumproben zeigten etwas unterschiedliche Kinetik bei der Wasserstoffkomplexbildung verglichen mit n-leitenden Siliziumproben. Die beobachteten Unterschiede führen $\mathrm{zu}$ verschiedenen Tiefenverteilungen der Wasserstoffplättchen in den beiden Siliziumsubstraten.

Mit der Raster-Channeling-Ionen-Transmissions Analyse wurde der Einfluss untersucht, den der Bruch im Ion-Cut Prozess auf die Kristallschäden hat. Diese Studie wurde am Abspaltungsprozess in Form von aufgebrochenen Oberflächenkratern durchgeführt. Es zeigte sich, dass der eigentliche Abspaltungsprozess auf mechanische Weise erhebliche Kristallschäden erzeugt.

Schliesslich wurde der Einfluss untersucht, den Boratome auf den Ion-Cut haben, die vor der Wasserstoffimplantation in das Silzium implantiert worden sind. Die Experimente wurden an n-leitenden Siliziumproben mit unterschiedlichen Dotierungskonzentrationen ausgeführt. Die Dotierungsniveaus wurden in einer Weise gewählt, dass die Auswirkung auf den Abspaltungsprozess untersucht werden konnte, die der Wechsel des Leitungstyps, die atomaren Wechselwirkungen zwischen the Bor- und Wasserstoffatomen, und die Variation des Fermi-Niveaus haben. Die Experimente zeigten auf, dass sowohl die atomaren Wechselwirkungen zwischen Wasserstoff und Bor als auch die Variation des Fermi-Niveaus einen erheblich beschleunigenden Effekt auf die Ansammlung von Wasserstoffmolekülen in Gasblasen haben, welche letztendlich die Abspaltung verursachen.

Diese Arbeit trägt zu einem besseren Verständnis des Ion-Cut-Phänomens bei und ermöglicht dadurch eine weitere Optimierung dieses Schichtabspaltungsprozesses. 



\section{Abstract}

The "Ion-Cut", a layer splitting process by hydrogen ion implantation and subsequent annealing is a versatile and efficient technique of transferring thin silicon surface layers from bulk substrates onto other substrates, thus enabling the production of silicon-oninsulator (SOI) materials. Cleavage is induced by the coalescence of the highly pressurized sub-surface $\mathrm{H}_{2}$-gas bubbles, which form upon thermal annealing. A fundamental understanding of the basic mechanisms on how the cutting process occurs is still unclear, inhibiting further optimization of the Ion-Cut process. This work elucidates the physical mechanisms behind the Ion-Cut process in hydrogen-implanted silicon. The investigation of the cleavage process reveals the cut to be largely controlled by the lattice damage, generated by the hydrogen ion irradiation process, and its effects on the local stress field and the fracture toughness within the implantation zone rather than by the depth of maximum H-concentration. Furthermore, this work elucidates the different kinetics in the H-complex formations in silicon crystals with different conductivity types, and examines the mechanically induced damage accumulation caused by the crack propagation through the silicon sample in the splitting step of the Ion-Cut process. Additionally, the influence of boron pre-implantation on the Ion-Cut in hydrogen implanted silicon is investigated. These studies reveal, that both, the atomic interaction between the boron implant and the hydrogen implant and the shift of the Fermi level due to the electrical activation of the implanted boron have a tremendous enhancing effect on the Ion-Cut process. 



\section{Contents}

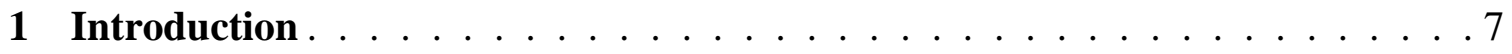

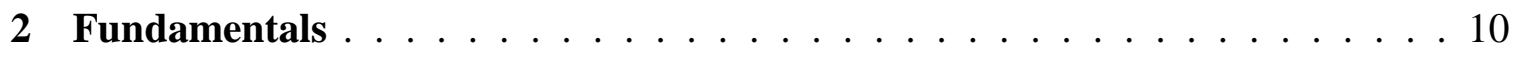

2.1 Formation of Silicon on Insulator by the Ion-Cut Process . . . . . . . . 10

2.2 The Silicon-Hydrogen System: A Literature Review . . . . . . . . . . . 12

2.3 Interactions of Ions with Condensed Matter . . . . . . . . . . . . . 17

3 Experimental: Ion Implantation . . . . . . . . . . . . . . . . . . 25

4 Experimental: Analyses Techniques . . . . . . . . . . . . . . . . 28

4.1 Composition Analysis . . . . . . . . . . . . . . . . . 28

4.1.1 Rutherford Backscattering Spectrometry (RBS) . . . . . . . . . 28

4.1.2 Elastic Recoil Detection (ERD) Analysis . . . . . . . . . . . . . 34

4.1.3 Secondary Ion Mass Spectroscopy (SIMS) . . . . . . . . . . . 42

4.2 Ion Beams for Crystal Damage Analysis . . . . . . . . . . . . . 45

4.2.1 RBS Channeling . . . . . . . . . . . . . . . . 45

4.2.2 Channeling Scanning Transmission Ion Microscopy (CSTIM) . . . . 51 
4.3 Surface Topography . . . . . . . . . . . . . . . . . . 53

4.3.1 Scanning Electron Microscopy . . . . . . . . . . . . . . 53

4.3.2 Atomic Force Microscopy . . . . . . . . . . . . . . . . 56

4.4 Microstructure: Transmission Electron Microscopy . . . . . . . . . . . . . . 58

4.5 Stress Measurements . . . . . . . . . . . . . . . . 61

5 The Mechanisms Behind the Ion-Cut Process . . . . . . . . . . . . . . . . 64

5.1 Low Dose Hydrogen Implantation . . . . . . . . . . . . . . 65

5.1.1 Implantation Damage Depth Profile before and after the Ion-Cut . . . 66

5.1.2 Depth Measurement of the Ion-Cut . . . . . . . . . . . 70

5.1.3 Microstructure of the Implantation Zone . . . . . . . . . . . . 73

5.1.4 H-depth distribution before and after the Ion-Cut . . . . . . . . . 79

5.1.5 Influence of Stress and Strain Field on H-Platelet Formation and Ion-Cut Location . . . . . . . . . . . . . . . . . . 85

5.2 Influence of the Hydrogen Implantation Dose on the Ion-Cut . . . . . . . . 92

5.2.1 Implantation Damage and Hydrogen Depth Profiles in the As-Implanted State . . . . . . . . . . . . . . . 93

5.2.2 Microstructure after the Heat Treatment . . . . . . . . . . . . . . 97

5.2.3 Influence of the Silicon Fracture Toughness on the Ion-Cut . . . . 106

5.3 Influence of the Sample Treatment on the Ion-Cut Location . . . . . . . 118

\section{Formation Kinetics of Hydrogen Complexes in Silicon and their Influence} on the Crystal Damage . . . . . . . . . . . . . . . . . . . . . . . . 132

6.1 Evolution of the Hydrogen and Implantation Damage Distribution upon Thermal Annealing . . . . . . . . . . . . . . . . 133

6.2 Growth Kinetics of Hydrogen Platelets . . . . . . . . . . . . . . . . . . 147

6.3 Mechanically Induced Damage during the Exfoliation of Individual Surface Blisters . . . . . . . . . . . . . . . . . 154 
7 Boron + Hydrogen Co-Implantation . . . . . . . . . . . . . . . . 160

7.1 Implantation Damage Depth Profiles . . . . . . . . . . . 163

7.2 Hydrogen and Boron Implantation Depth Profiles . . . . . . . . . . 167

7.3 SEM Analysis of the Surface Blistering . . . . . . . . . . . . . 173

7.4 The Influence of the Pre-Implanted Boron on the Surface

Blister Formation . . . . . . . . . . . . . . . . . 177

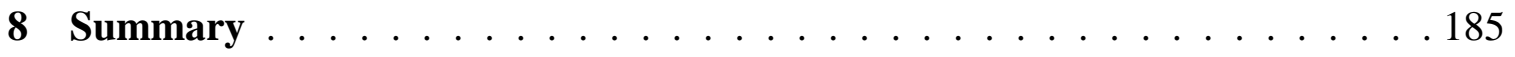

Bibliography . . . . . . . . . . . . . . . . . 188 



\section{Introduction}

The development of a new technology for the integration of dissimilar materials will be needed to meet many of the advanced technological needs of the future. Current technologies are limited by the ability to integrate materials systems together on a single platform (e.g., a $\mathrm{Si}$ wafer for applications in microelectronic, optoelectronic, and microelectromechanical systems - MEMS). If materials processes were developed to overcome this barrier, then new microelectronics, three-dimensional electronics, and the integration of small mechanical systems made from dissimilar materials would become standard.

One possible method to achieve this multi-material integration may be through the use of Ion-Cut processing. This layer splitting process by ion-implantation and subsequent annealing is an innovative approach for engineering materials that provides an effective, versatile, and economical method to transfer thin surface layers from bulk substrates onto a host of other substrates. The essence of the Ion-Cut process is to preimplant the device wafer with high dose gas atoms at a well defined depth before joining to a handle wafer. Then, on subsequent annealing of the joined pair complete shearing (or 'exfoliation') of the device wafer occurs in the gas ions implanted region. The IonCut process can be applied to a variety of materials, including germanium [1], siliconcarbide [1,2], diamond thin films [1], or the production of silicon on glass [3].

One of the most promising applications of the Ion-Cut is the production of silicon-on-insulator (SOI) materials by proton implantation into crystalline silicon. Silicon-on-insulator (SOI) technology [4] is a powerful method for fabricating deep submicrometer complementary metal-oxide silicon devices (CMOS). Recent advances in SOI technology has generated increased interest in their application to high speed CMOS circuits. The speed advantage is a direct consequence of the insulating layer, which

provides isolation from the substrate thereby decreasing capacitances for both devices and electrical interconnections. 
Exfoliation of silicon layers by hydrogen ion implantation and subsequent heat treatment is an effect that has been known for more than two decades and has been used to experimentally determine ion ranges. [5,6] Fifteen years later Bruel et al. discovered the potential significance of this effect in terms of a promising application of the Ion-Cut process. He and his co-workers developed a new technology for the production of silicon on insulator (SOI), called Smart-Cut ${ }^{\mathrm{TM}}$. [7-9] In this work I will refer to the Smart-Cut ${ }^{\mathrm{TM}}$ process by the more generic term 'Ion-Cut' process, which is used in the literature. The Ion-Cut process consists of four steps: (i) proton implantation into a silicon wafer, (ii) RCA clean of the bond surfaces, (iii) hydrophilic bonding of the implanted wafer to another substrate, a $\mathrm{SiO}_{2}$ capped silicon wafer, and (iii) annealing of the bonded wafers. During the heat treatment the $\mathrm{H}$-implant evolves into highly pressurized $\mathrm{H}_{2}$ gas bubbles which grow in size and ultimately lead to a crack propagation throughout the whole hydrogen-implanted silicon wafer. [10,11]

The development of the Ion-Cut provides for the first time a methodology for producing ultra thin silicon layers required for state-of-the-art CMOS devices. Over the last two decades, SOI technology has relied on two methods known as "wafer-bonding and etch-back SOI" (BESOI) and "separation by implantation of oxygen" (SIMOX). The Ion-Cut offers many benefits over BESOI and SIMOX: Because in Ion-Cut the oxide layer is thermally produced, the buried oxide layer is of high quality and uniform thickness. The SOI structure is produced in one step without the need of costly grinding and polishing or etchback. Furthermore, the hydrogen-induced fracturing of the silicon wafer leaves an extremely uniform, nearly defect free silicon layer bonded to the handle wafer, requiring only a moderate polish to achieve a 1-2 Angstrom rms (root mean square) roughness necessary for further processing. The thickness of the exfoliated silicon layer is very uniform. [12] In addition, the silicon wafer, from which the hydrogen implanted layer is exfoliated, can be reused.

The Ion-Cut process was developed by an engineering approach, with poor understanding of the fundamental mechanisms that drive the exfoliation, impeding further optimization. Although, an immense amount of research has been conducted to achieve knowledge about how hydrogen interacts with silicon at the atomic level [13], a fundamental understanding of the basic mechanism on how the cutting process occurs is still unclear. Hence, further studies are needed to develop the Ion-Cut method into a viable industrial technique.

The aim of this work was to examine the mechanisms behind the exfoliation process in hydrogen implanted silicon. Chapter 2 presents a short overview of the fundamentals of the silicon-hydrogen system, describes the Ion-Cut process in more 
detail, and gives a short review of the fundamentals of ion-solid interactions. The following two chapters introduce the experimental methods used in this work. Chapter 3 describes the experimental setup for the sample preparation (i.e. ion-implantation), whereas chapter 4 presents the experimental analysis techniques applied for the analysis of the samples.

The results of this work are listed in chapter 5 to 7 . Chapter 5 present the results deduced from Ion-Cut samples produced under various implantation parameters. From these results the influence of the implantation damage and the $\mathrm{H}$-ion implantation dose on the Ion-Cut could be revealed and the physical mechanisms behind the Ion-Cut process were elucidated. Chapter 6 discusses the influence of the silicon dopant type (p- or ntype) and annealing temperature on the behavior of the H-implant in terms of implantation damage evolution and hydrogen platelet formation. The enhancement of the Ion-Cut by boron pre-implantation of proton implanted silicon is subject of chapter 7 . Finally, chapter 8 summarizes the gained knowledge about the mechanisms behind the hydrogen implantation induced silicon surface layer cleavage. 


\section{Fundamentals}

This chapter provides a literature review and a brief description of the fundamental technological and physical background related to this work. First, the Ion-Cut based production of SOI is explained. Then, a short overview of the knowledge about the silicon-hydrogen system is portrayed. The last part of this chapter presents the physics associated with the penetration of energetic ions into solids.

\subsection{Formation of Silicon on Insulator by the Ion-Cut Process}

The Ion-Cut process uses ion implanted gas atoms, such as $\mathrm{H}$, to promote cleavage of thin layers surface layers which are transferred from bulk substrates onto a host of other substrates. The application of this phenomenon on hydrogen implanted silicon led to the development of the Smart-Cut ${ }^{\mathrm{TM}}$ [7-9], a new technology for the production of silicon on insulator (SOI). The process is schematically illustrated in figure 2-1.

Hydrogen is introduced into a single crystal silicon wafer by $\mathrm{H}$-ion implantation to a well-defined depth. After the ion-implantation, the surfaces of the implanted silicon wafer and another silicon wafer capped with a silicon dioxide layer (the so-called "stiffener" or "handle wafer") undergo a modified RCA clean, a surface cleaning process, developed by the Radio Corporation of America. This step is necessary to remove surface contaminations introduced by the $\mathrm{H}$ ion-implantation process. The cleaning of the wafer surfaces prior to the bonding must be carried out very carefully to leave both surfaces free of particle or organic contaminations. The cleaning procedure results in hydrophilic clean oxide surfaces on both substrates. [14] The substrate surfaces are then bonded together. When the two substrates are pressed together, interactions between water adsorbed on both surfaces lead to weak bond between the wafers via hydrogen 
bonds. [15] Next, the joined pair is heated to about $200{ }^{\circ} \mathrm{C}$ for a few hours to establish a strong chemical bond between the two wafers.

1) H ion-implantation

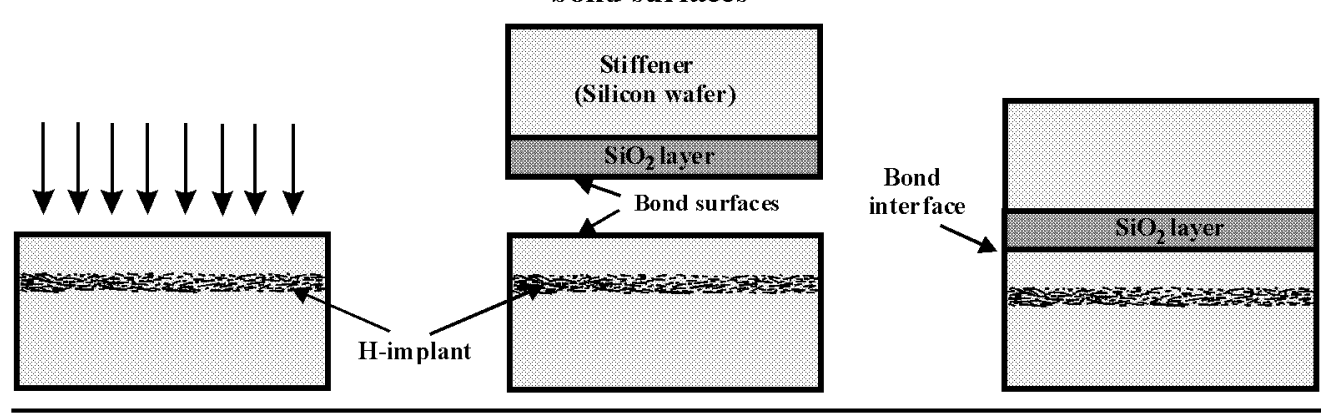

4) Annealing

$\rightarrow$ Exfoliation

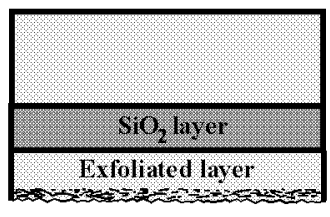

Donor wafer
2) RCA clean of bond surfaces

5) Slight Polish
3) Wafer bonding
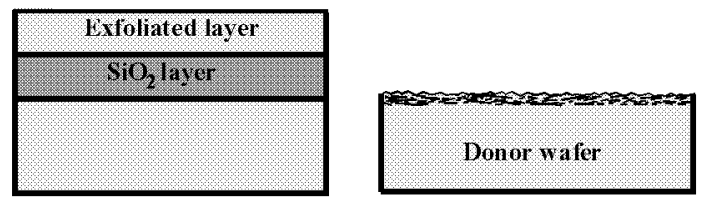

Figure 2-1: Schematic illustration of the Ion-Cut process for the production of SOI.

After a reasonable bond strength between the wafers is achieved, the joined pair undergoes an anneal at elevated temperatures of typically $400{ }^{\circ} \mathrm{C}$. During this heat treatment, the implanted hydrogen atoms rearrange within the implantation zone, forming $\mathrm{H}_{2}$-gas bubbles of high internal pressure. Raman spectroscopy experiments on samples used in this work measured a $\mathrm{H}_{2}$-gas pressure of up to $50 \mathrm{kbar}$. The larger and energetically more favorable gas bubbles grow in size at the expense of smaller gas bubbles via Ostwald ripening. [16] Finally, at a critical anneal duration or anneal temperature the $\mathrm{H}_{2}$-gas bubbles coalesce and lead to the propagation of a crack through the whole silicon crystal. [9] The last step comprises a slight polish of the Ion-Cut surface to remove the damage layer, which results from the $\mathrm{H}$ ion-irradiation process.

This work focuses on the examination of the cleavage step in the Ion-Cut process for hydrogen-implanted silicon. 


\subsection{The Silicon-Hydrogen System: A Literature Review}

The physics and chemistry of hydrogen in silicon has been the subject of considerable scientific and technological interest for over three decades. This interest has been driven by the omnipresent appearance of hydrogen in silicon processing which always leads to hydrogen incorporation into the substrate, either intentionally or unintentionally. Importantly, this hydrogen may strongly alter the electrical characteristics of the resultant device by diffusion into active region and passivation of the dopant. Attention has been focused primarily, although not exclusively, on the diffusion of atomic hydrogen, its molecule formation, and its complex formation with and passivation of dopant impurities. This chapter provides a brief literature review of the studies performed on the siliconhydrogen system.

The diffusion of $\mathrm{H}$ in $\mathrm{Si}$ is complex because of the presence of several charge states and the fact that hydrogen in often present in different forms, namely atomic, molecular (or larger clusters), or bound to a defect or impurity. The probability for the formation of these different forms is dependent on the defect and impurity concentration in the material and the hydrogen concentration itself. Thus, the apparent diffusivity is dependent on the method of hydrogen insertion. Hydrogen can be introduced into silicon through various processes like reactive ion-etching, glow discharge, plasma hydrogenation, or H-ion implantation and consequently each method with its distinct influence on defect or impurity generation in the silicon crystal leads to a different behavior of hydrogen in the material. For example, hydrogen appears to diffuse more rapidly under conditions of low hydrogen concentration than it does under conditions of high concentration, such as plasma exposure. Furthermore, H-diffusion turned out to be a function of the silicon conductivity and type.

The earliest work on the behavior of hydrogen in crystalline silicon is that of Van Wiering and Warmholz (1956) who studied the diffusion of hydrogen through the walls of cylinders made of single crystal silicon at high temperatures between 900 and $1200{ }^{\circ} \mathrm{C}$. [17] Their early high temperature permeation experiments found that hydrogen is very mobile in c-Si and has a diffusion coefficient of

$$
D_{H}=9.4 \cdot 10^{-3} \cdot \exp \left(\frac{-0.48 \mathrm{eV}}{k T}\right) \cdot \mathrm{cm}^{2} \cdot \mathrm{s}^{-1}
$$


where $k$ is the Boltzmann constant. About ten years later, Ichimiya and Furuichi found, that in the temperature range of 400 to $550{ }^{\circ} \mathrm{C}$ tritium diffusion in crystalline silicon could be described as [18]

$$
D_{T}=4.2 \cdot 10^{-5} \cdot \exp \left(\frac{-0.56 \mathrm{eV}}{k T}\right) \cdot \mathrm{cm}^{2} \cdot \mathrm{s}^{-1}
$$

Experimental and theoretical studies on H-diffusion in silicon at lower temperatures, where trapping of hydrogen at defects and impurities and $\mathrm{H}_{2}$-molecule formation are significant, discovered much lower effective diffusivities [19-30]. Values for the Hdiffusion coefficient in silicon expected from an extrapolation of the diffusion coefficient of equation (2-1) to lower temperatures are several orders of magnitude higher than experimentally obtained diffusivities. This is illustrated in figure 2-2, which shows as an example a comparison of theoretical predicted diffusion coefficients of $\mathrm{H}^{0}$ and $\mathrm{H}^{+}$in $\mathrm{p}$ type silicon at low temperatures [22] to the extrapolation of the H-diffusion coefficients from equation (2-1) and (2-2) to low temperatures.

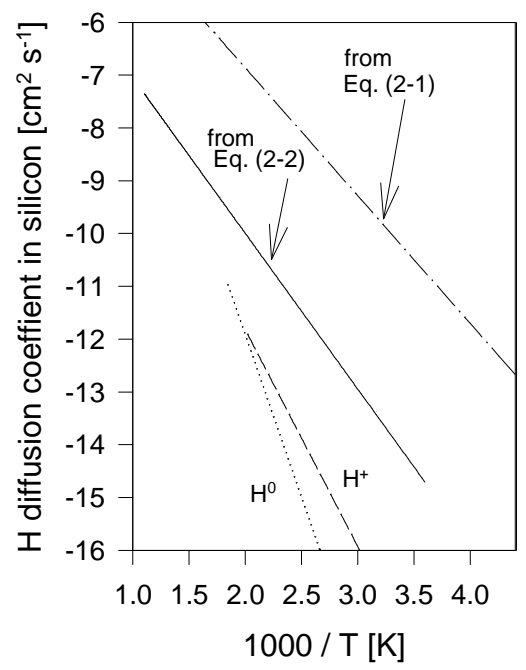

Figure 2-2: Arrhenius plot of expected H-diffusivity from Van Wiering and Warmhotz [17] (Eq. 2-1), Ichimiya and Furuichi [18] (Eq. 2-2) by extrapolation to low temperatures, and the diffusivities of neutral and positive charged $\mathrm{H}$ atoms predicted by Capizzi and Mittiga [22].

Several processes have been invoked in order to explain the details of observed hydrogen profiles in silicon. These processes include reactions of hydrogen at dopant sites and with lattice defects, reactions between hydrogen atoms, and dissociation of trapped hydrogen. Pseudopotential density functional calculations showed that atomic hydrogen in silicon can appear in all three charge states $\mathrm{H}^{+}, \mathrm{H}^{0}$, and $\mathrm{H}^{-}$. [31] The charge state is dependent on the position of the Fermi level. The positive charge state is more stable in p-type silicon, the negative charge state more stable in highly n-type doped silicon, and hence, also charge state dependent diffusion coefficients have been incorporated into many kinetic studies. 
Ion-Implantation into crystalline silicon breaks chemical bonds and creates point defects in the implantation zone. The fundamental mechanisms of how this occurs will be discussed in chapter 2.3. This effect is crucial in the Ion-Cut process: Since the diffusivity of $\mathrm{H}$ in crystalline silicon is very high, one would expect intense out diffusion of the H-implant during the heat treatment of the silicon sample. However, the hydrogen gets trapped at the proton implantation induced defects by passivating the dangling bonds in the implantation zone, where the number of lattice defects is very high.

As an example, figure 2-3 illustrates possible local $\mathrm{Si}-\mathrm{H}$ defects, formed at a silicon monovacancy. A vacancy in the silicon lattice leaves four dangling bonds behind, which can be passivated with $\mathrm{H}$-atoms. Also shown is the so-called $\mathrm{H}_{2}{ }^{*}$ defect, a commonly observed $\mathrm{H}_{2}$ complex in hydrogen implanted silicon.

Silicon

○ Hydrogen
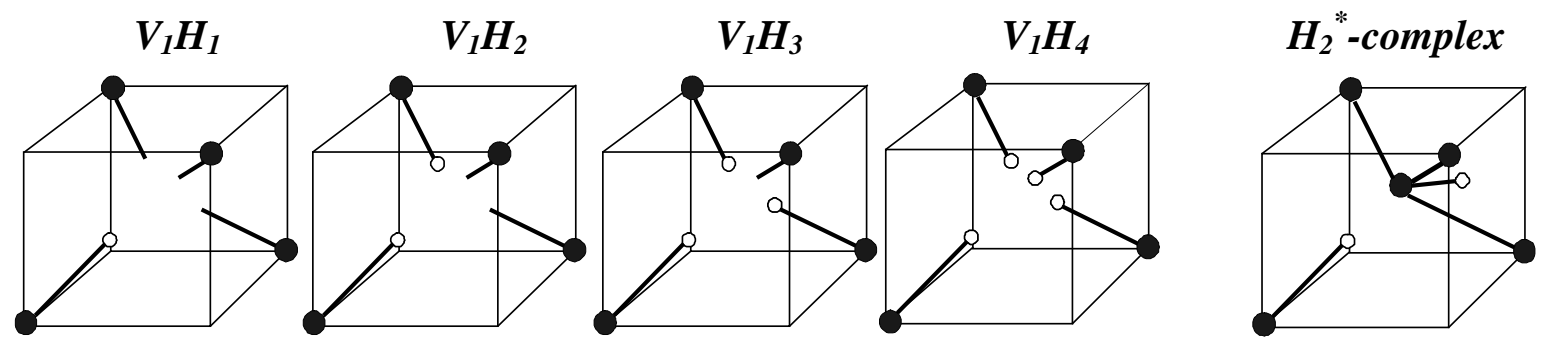

Figure 2-3: Illustration of $\mathrm{Si}-\mathrm{H}$ defects observed in $\mathrm{H}$-ion implanted silicon. $\mathrm{V}$ denotes a vacancy and $\mathrm{H}$ denotes a H-atom.

Hydrogen in crystalline silicon has been of great theoretical and experimental interest in recent years. A lot of advances in the theoretical and experimental research of the hydrogen silicon system in terms of hydrogen complex formation with silicon and impurity atoms and hydrogen molecule formation were achieved (see for example $[32,33])$. Many insights about hydrogen complex formation in $\mathrm{H}$-ion-implanted silicon have been gained from IR vibrational spectroscopy. A multitude of vibrational bands in silicon that contain $\mathrm{H}$ were found, far more than the modes attributed to $\mathrm{Si}-\mathrm{H}, \mathrm{Si}-\mathrm{H}_{2}$, and $\mathrm{Si}-\mathrm{H}_{3}$. Stein at al. showed, that $\mathrm{Si}-\mathrm{H}$ bonds associated with ion implantation induced vacancy and interstitial-related displacement damage are less stable than surface traps of hydrogen at internal voids. [34] This is in accordance with the evolution of $\mathrm{Si}-\mathrm{H}$ complexes into $\mathrm{H}_{2}$-gas bubbles upon annealing.

Detailed information about the evolution of $\mathrm{Si}-\mathrm{H}$ complexes in $\mathrm{H}$ ion-implanted silicon were gained by infrared vibrational studies by Weldon et al. and Chabal et al. $[35,36]$. Implanted $\mathrm{H}$ atoms form complexes of the form $V_{x} H_{y}$ or $I_{x} H_{y}$ where $V$ denotes a 
silicon vacancy and $I$ denotes a silicon interstitial, and the subscript have values of $\mathrm{x}=1$ and 2 and $\mathrm{y}=1-4$. Also observed was the so-called $\mathrm{H}_{2}{ }^{*}$ complex, a hydrogen molecule formation, where one $\mathrm{H}$ atom is located at the bond centered site and the other at the antibond site with a silicon lattice atom residing in between the $\mathrm{H}_{2}$ bond (see right image in figure 2-3). (Nielsen et al. and Holbech et al. performed detailed studies on this species. [37-39]) Upon annealing of the $\mathrm{H}$ ion-implanted samples, the IR studies uncover a net loss of bound hydrogen and agglomeration of hydrogen at existing vacancies. A comparison of the infrared vibrational studies with elastic recoil detection analysis, an analysis method which monitors not only atomic hydrogen but also molecular hydrogen, revealed that a net conversion from $\mathrm{Si}-\mathrm{H}$ complexes into $\mathrm{H}_{2}$ takes place at elevated temperatures. This is in agreement with the observations of $\mathrm{H}_{2}$ filled gas bubbles in the implantation zone.

Earlier investigations led to discover the astonishing result that $\mathrm{H}$ can insert itself between $\mathrm{Si}-\mathrm{Si}$ bonds at the bond-centered site to form extended structural defects that are described as hydrogen stabilized platelets. [40] These H-platelets turn out to be the nuclei of the $\mathrm{H}_{2}$-gas bubbles, which lead to ion-cutting in $\mathrm{H}$-implanted material. This will be discussed in detail in chapter 5. Recent experimental studies suggest, that the nucleation of H-platelets is solely controlled by the Fermi energy. [41]

Cerofolini et al. hypothesized, that the $\mathrm{H}_{2}$ molecules that formed within these bubbles do so with a bond length almost equal the $\mathrm{H}_{2}$ bond length in vacuum, which leads to an energy gain that counterbalance the strain build up around the bubble in the silicon crystal. [42] Recent Raman spectroscopy measurements verified this assumption. The vibrational frequency of $\mathrm{H}_{2}$ molecules is practically the same as the well-known value for gaseous hydrogen. [43]

The interaction of hydrogen in silicon with acceptor or donor dopants turned out to have a considerable influence on the kinetics in the formation of $\mathrm{H}$-platelets and $\mathrm{H}_{2}$-gas bubbles, which induce the Ion-Cut.

Studies of neutralization of shallow acceptors in silicon evolved from the perspective that there is a $\mathrm{Si}$ dangling bond near each acceptor, and therefore its passivation should eliminate a hole and result in an increased resistivity. First studies of the interaction of hydrogen with acceptors in silicon showed the neutralization of boron by monatomic hydrogen to be maximized near $100{ }^{\circ} \mathrm{C}$, being limited by diffusion at lower temperatures and by the dissociation of $\mathrm{Si}-\mathrm{H}-\mathrm{B}$ complex at higher temperatures. [44-47] 
Hydrogen in-diffusion experiments into crystalline silicon with a buried layer of high boron concentration showed significant trapping of hydrogen within the boron doped layer. [48] The amount of H-atoms trapped exceeds by far the amount of boron atoms present in the layer. Theoretical studies showed, that the change of the Fermi level, induced by the boron concentration profile has an influence on the charge state. Also, kinetic models for hydrogen passivation of the acceptors indicated that the hydrogen boron capture radius is large, and that molecular formation of $\mathrm{H}$ at the boron trap site lead to the trapping of 8 to 12 hydrogen atoms by one boron atom in the silicon crystal. [49] Theoretical studies confirmed the multiple trapping of hydrogen atoms at boron in silicon. [50] However, details about the trap mechanisms are still unclear.

For the first time Johnson and co-workers reported that shallow donor dopants in n-type c-Si could also be neutralized by hydrogenation, although not as effectively as with boron. [51] Since the first report, several experimental studies have confirmed the existence of donor-hydrogen complexes. [52] Studies on the kinetics of the thermal dissociation of hydrogen-donor complexes established the existence of a negative charge state for diffusing hydrogen in silicon. [53]

In summary, the research on the hydrogen silicon system showed the apparent diffusivity of atomic hydrogen in the temperature range up to 300 to $400{ }^{\circ} \mathrm{C}$ approaches the values from Van Wiering and Warmholz relation only under conditions of low hydrogen concentration and low impurity contents. This is due to the lower probability of self trapping of hydrogen by molecular or H-platelet formation, and the lower probability of hydrogen to get trapped by lattice defects, and acceptor or donor dopant ions. Particularly, the research conducted on hydrogen complex formation in silicon in the last few years was essential in interpreting the phenomena discovered in this work. 


\subsection{Interactions of Ions with Condensed Matter}

Ion Implantation is a process by which virtually any element can be injected into the near-surface region of any solid by causing a beam of high-velocity ions to strike a target. All samples in this work were prepared by $\mathrm{H}$-ion or B-ion implantation into crystalline silicon. Knowledge about the interaction of low energetic ions with condensed matter is needed to understand the defect generation in crystalline silicon by the ion-implantation process. Knowledge about the interaction of high energetic ions with condensed matter is needed to understand how the depth scales are established in the ion-beam analyses of the silicon samples. This chapter reviews the physics associated with the penetration of energetic ions into solids. It provides a brief description of the quantitative evaluation of how the ions lose energy to the solid and of the resulting depth concentration profile of implanted ions after they stop within the solid. Defect generation by the ion-irradiation process and the resulting implantation damage depth distribution are explained as well.

An energetic ion penetrating a target interacts with the target atoms by two major processes. It transfers energy to the target by elastic nuclear collisions and inelastic electronic energy loss processes. The energy loss rates per length unit resulting from the two different mechanisms are called nuclear and electronic stopping, respectively. The relative importance of the various interaction processes between the ion and the target medium depends mostly on the ion velocity and on the charges of the ion and target atoms. The total energy loss is obtained as a sum of the nuclear and electronic contributions.

The theoretical treatment of the stopping of ions in matter is due greatly to the work of Bohr [54-56], Bethe [57-59], Bloch [60,61] Firsov [62,63] and Lindhard et al. [64-67]. For the advanced study of ion-solid interaction, the reader is referred to literature. [68-70]

\section{Nuclear stopping}

The nuclear stopping results from the average energy loss by elastic collisions of the incoming ion with target atoms. Assuming the incoming ion and the target atom with which the ion interacts to be isolated simplifies the interactions to a two-body collision event. Figure 2-4 shows the kinematics of such a two-body collision in the center of mass frame. 


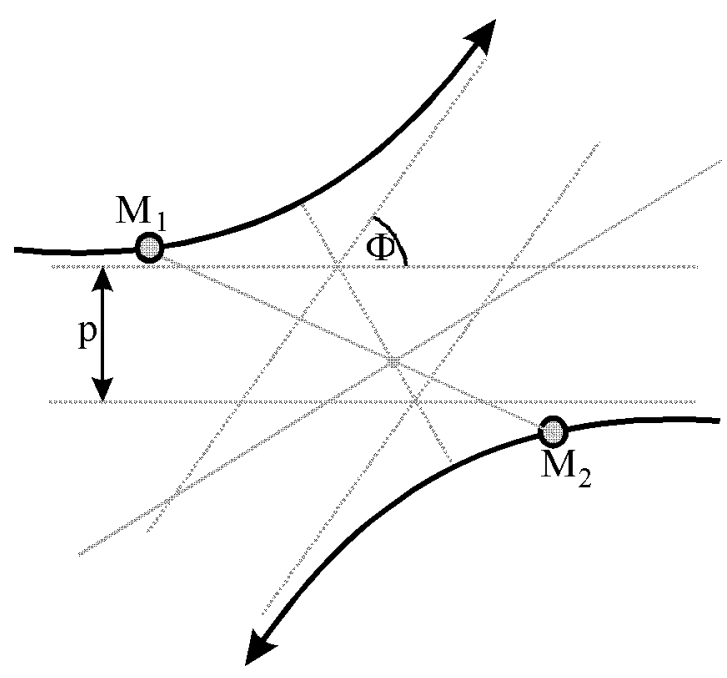

Figure 2-4: The collision path for a nuclear collision of the incoming ion with the nucleus of a target atom in center of mass coordinates.

In the laboratory frame, where the kinetic energy of the incoming ion with mass $M_{1}$ and velocity $v_{1}$ is $E_{1}=1 / 2 M_{1} v_{1}^{2}$, whereas the target atoms stands still before the collision, the kinetic energy transferred to the target atom can be easily calculated to be:

$$
E_{\text {Transfer }}=\frac{1}{2} M_{2} v_{2}^{2}=\frac{4 M_{1} M_{2}}{\left(M_{1}+M_{2}\right)} \cdot E_{1} \sin ^{2}(\Phi / 2)
$$

where $M_{2}$ is the mass of the target atom, $v_{2}$ the velocity of the target atom after the collision event and $\Phi$ is the collision angle. The distance $b$ of closest approach (see fig. $2-3$ ) is the important kinematic parameter and is given by

$$
b=\frac{2 Z_{1} Z_{2} e^{2}}{v_{1}^{2}} \cdot \frac{M_{1} M_{2}}{M_{1}+M_{2}}
$$

with $Z_{1} e$ and $Z_{2} e$ as the charges of the incoming ion and the target nucleus, respectively. The impact parameter $p$ relates to $b$ as:

$$
p=b \cos (\Phi / 2)
$$

The relationship between the energy transfer $E_{\text {Transfer }}$ and the distance $b$ depends on the interaction potential. The integration over the differential energy transfer over all impact parameters gives the nuclear stopping power, i.e. the energy lost by the ion per unit length, $S_{n}=-(d E / d x)_{n}$ which is

$$
S_{n}\left(E_{0}\right)=\int_{0}^{\infty} E_{\text {Transfer }}\left(E_{0}, p\right) 2 \pi p d p=2 \pi \frac{4 M_{1} M_{2}}{\left(M_{1}+M_{2}\right)^{2}} \int_{0}^{p_{\max }}\left(\sin \frac{\Phi}{2}\right) p d p
$$

where $E_{0}$ is the initial kinetic ion energy. The scattering angle $\Phi$ is a function of the interaction potential V(r). To quantify the energy transfer by nuclear interaction of the ion with the target atoms, it is necessary to specify an interaction potential. In earlier years 
analytical functions for $\mathrm{V}(\mathrm{r})$ were chosen, but with increasing computing power more complicated and non-analytical functions were introduced which provide closer agreement between theory and experiment. For instance screened Coulomb potentials of the form:

$$
V(r)=\frac{Z_{1} Z_{2}}{r} \exp (\Phi(r / a))
$$

with various functions $\Phi(a)$ were chosen from Brinkman, Firsov, Lenz-Jensen and Lindhard (see [71-73]). The screening parameter $a$ is related to the Bohr radius $a_{0}$ of 0.529 Angstrom by function like:

$$
a=\frac{0.8854 a_{0}}{Z_{1}^{0.23}+Z_{2}^{0.23}}
$$

where the exponent is semi-empirical. The first unified approach to nuclear stopping was made by Lindhard, Scharff and Schiott [64] and is commonly called the LSS theory. They found the so-called reduced nuclear stopping power to be:

$$
\begin{array}{ll}
S_{n}(\varepsilon)=\frac{\ln (1+1.1383 \varepsilon)}{2\left[\varepsilon+0.01321 \varepsilon^{0.21226}+0.19593 \varepsilon^{0.5}\right]} & (\varepsilon \leq 30) \\
S_{n}(\varepsilon)=\frac{\ln (\varepsilon)}{2 \varepsilon} & (\varepsilon>30)
\end{array}
$$

In modern semi-empirical formulations the potential has been adjusted so that the nuclear stopping power provides accurate fits with experimental data.

\section{Electronic stopping}

The electronic collisions involve much smaller energy losses per collision, negligible deflection of the ion trajectory, and negligible lattice disorder. [70] The origins of the electronic energy losses are kinetic energy transfer to target electrons by electron-electron interactions, excitations of band electrons and conduction electrons, ionization of target atoms, and excitation, ionization or electron-capture of the incoming ion itself.

The calculation of the electronic energy loss of incoming ions can be divided into two categories, i. e. one for light and one for heavy ions. For this work, only ion- 
implantations with light ions were performed: $\mathrm{H}$ and $\mathrm{B}$ ion implantation into silicon in the $\mathrm{keV}$ energy range for sample preparation and $\mathrm{He}$ ion implantation in the $\mathrm{MeV}$ energy range for ion beam analysis (see chapter 4.1 and 4.2). Therefore, a brief description of electronic stopping for light ions given:

At ion energies less than $2 \mathrm{MeV} / \mathrm{Z}_{1}$ the electronic stopping power can be calculated by the Lindhard-Scharff approach [74], which suggested that the electronic stopping power is proportional to the ion velocity and can be expressed in terms of the reduced electronic stopping power $S_{e}$ as

$$
S_{e}=K_{L} \varepsilon^{1 / 2}
$$

with $\varepsilon$ defined in equation (2-11) and

$$
K_{L}=\frac{0.0793 \cdot Z_{1}^{2 / 3} Z_{2}^{1 / 2}\left(M_{1}+M_{2}\right)^{2 / 3}}{\left(Z_{1}^{2 / 3}+Z_{2}^{1 / 2}\right)^{3 / 4} M_{1}^{3 / 2} M_{2}^{1 / 2}}
$$

The electronic stopping power will decrease, when the ion velocity exceeds the orbital velocity of the target electrons. It is then treated by the Bethe Bloch theory and can be described as

$$
S_{e}(\varepsilon)=\frac{4 \pi e^{4} Z_{1}^{2} Z_{2}}{m_{e} v^{2}} L(v)
$$

with Bethe's stopping number:

$$
L(v)=\ln \left(\frac{2 m_{e} v^{2}}{I}\right)-\ln \left(1-\frac{v^{2}}{c^{2}}\right)-\frac{v^{2}}{c^{2}}-\frac{c}{Z_{2}}-\frac{\delta}{2}
$$

where $I$ is the target mean excitation potential, $c / Z_{2}$ a shell correction and $\delta$ an ultra relativistic density correction. The mean excitation potential is defined as

$$
\ln I=\sum_{n} f_{n} E_{n}
$$

where $E_{n}$ are all possible energy transitions of the target atom and $f_{n}$ the corresponding dipole oscillator strengths.

Closely correlated to the electronic stopping power is the charge state of the incoming ion. As long as the orbital velocity exceeds the ion velocity, the electrons will stick to the incoming ion. Slower electrons, belonging to the outer shells, will be stripped. Bohr estimated the average ion charge to increase with the ion velocity. However, the incoming ion will reach its equilibrium charge distribution after passing a very short distance, independent on its initial charge state. 
The relative importance of the two energy-loss mechanisms changes considerable with the energy and atomic $\mathrm{Z}$ number of the incoming ion. Electronic stopping predominates at high kinetic ion energies and low $\mathrm{Z}$ numbers, while nuclear stopping takes over at low kinetic energies and high $\mathrm{Z}$ numbers. Figure 2-5 shows as an example the rates of energy transfer for electronic and nuclear stopping for protons penetrating silicon, the element system, used mostly in this work.

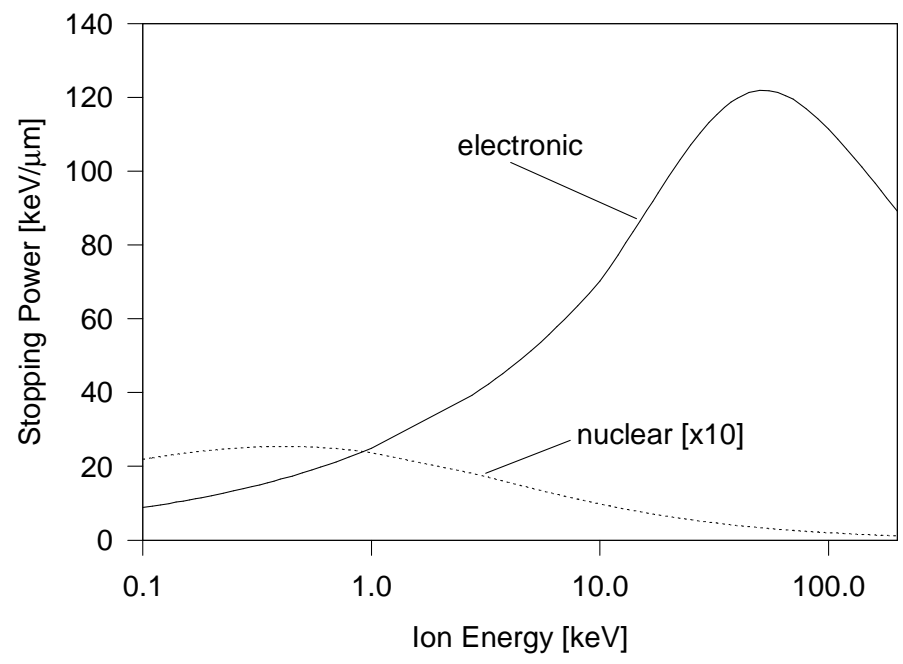

Figure 2-5: A comparison of the rates of energy transfer for the electronic stopping (solid line) and the nuclear interaction (dashed line) of a $\mathrm{H}$-ion entering a silicon target. The simulation was performed by the TRIM computer code [75]. Note that the nuclear stopping is multiplied by a factor of 10 and the energy axis is logarithmic.

At low kinetic ion energies, the nuclear and electronic stopping powers are competitive. The nuclear stopping power reaches its maximum in the keV energy range and follows the classical Rutherford distribution at higher energies. At velocities $\geq Z_{1}^{2 / 3} e^{2} \hbar$, the electronic stopping power dominates according to equation (2-12). In this energy regime the incoming ion is mostly neutral and the electronic stopping is proportional to the ion velocity. Beyond its maximum the ion is practically stripped from its electrons and the electronic stopping power follows the Bethe regime (equation 2-12) and decreases with increasing ion energy.

\section{Ion range distribution:}

Knowing the nuclear and electronic stopping powers for an ion traversing a target, the mean penetrated path length $R\left(E_{0}\right)$ of an ion before coming to rest can be calculated as

$$
R\left(E_{0}\right)=\int_{E_{0}}^{0} \frac{d E}{S_{n}(E)+S_{e}(E)}
$$

Because the stopping of an ion is a stochastic process, the collision sequence and the subsequent ion deflection, and the ion's total path length in coming to rest vary randomly 
between the individual ions. As a results, ions with the same energy, incident with the same angle onto the sample surface, and into the same material, do not necessarily have the same range. Instead, the depths to which the ions penetrate are statistically broad. The distribution in projected ranges is referred to as the range distribution or range straggling, with the most probable projected range referred to as the mean projected range. Because of ion scattering, the ion range normal to the target surface, denoted as the mean projected range $R_{p}\left(E_{0}\right)$, is smaller than the mean penetrated path length by a factor which depends on the scattering angles, and hence on the specific path of an individual ion. This is illustrated in figure 2-6.

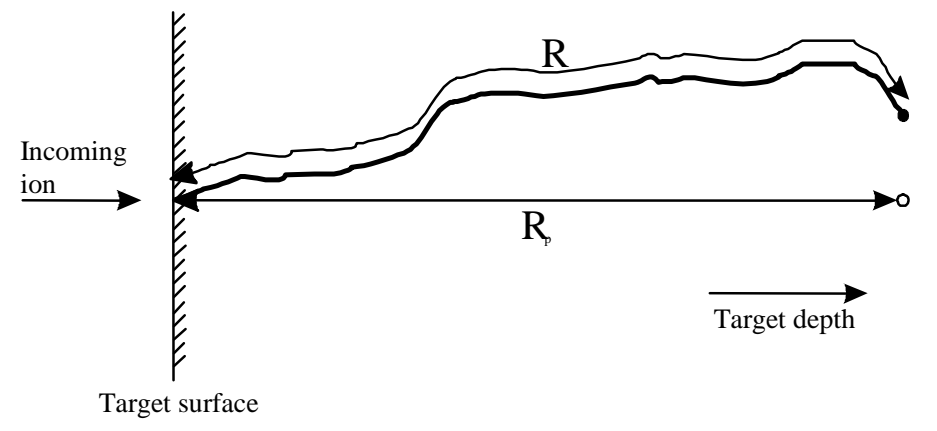

Figure 2-6: Schematics of the total path length and the projected range of an incident ion. (after [68])

For a single element target the ratio $R_{p} / R$ is $<<1$ for $M_{1} \ll<M_{2}$ and close to 1 for $M_{1} \gg M_{2}$. The statistical fluctuations in the stopping mechanisms will lead to a spread of the range $\mathrm{R}$ and thus will add to the spread $\Delta R_{p}$. The degree of $\Delta R_{p}$ increases with increasing penetration depth of the ions. The straggling results in a final ion depth distribution which can be approximated in the simplest case by a Gauss-distribution.

An approximation of the projected range can be found using the theory of Lindhard, Scharff and Schiott, which gives

$$
\frac{R}{R_{p}} \cong 1+B \frac{M_{2}}{M_{1}}
$$

where $B$ is a slowly varying function of the kinetic ion energy and the mean path length $R$. At higher ion energies, the increased electronic stopping power leads to smaller values for $B$.

The mean projected range can also be found by implementing computer simulations of the ion implantation process by the Monte Carlo approach. 


\section{Implantation damage generation}

During its path through the crystal the ions undergo a number of collisions with target atoms before they come to rest. If the energy transferred to the target atom is higher than the energy, required to move the target atom out of the potential well, which represents its stable lattice site, the lattice atom will be displaced. In the simplest case, a Frenkel pair is created, i.e. the atom left a vacancy behind and occupies an interstitial lattice site. Such a target atom displaced by an incident ion is called "primary knock-on atom" or PKA. The PKAs can in turn displace other target atoms by secondary knock-ons, tertiary knock-ons etc. Thus, if the implantation energy is high, each implanted ion causes displacement cascades within the crystal lattice. Such events will result in many collisions and displacements events occurring in near proximity of each other. Figure 2-7 illustrates the formation of such a collision cascade.

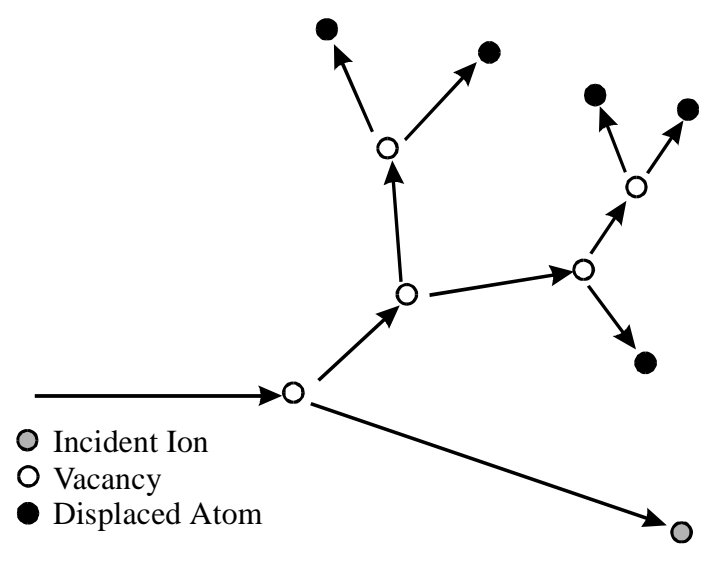

Figure 2-7: Schematic of the formation of a collision cascade by a PKA atom.

As the ion dose increases, the individual damage cascades will overlap and form a damaged layer in the crystal.

To calculate the damage created by the ion-implantation process, the partition of energy loss between electronic and nuclear processes must be determined. Only nuclear interaction of energetic incoming ions with target atoms result in PKAs, which lead to the radiation damage effects. Since the nuclear interaction of the incoming ions with target atoms predominates only at low energies, the creation of PKAs becomes significant shortly before the ions come to rest in the material. The partition of energy loss between electronic and nuclear processes was used in determining the range distribution of implanted atoms. The difference between ion range and crystal damage calculation is that in the latter case the energy partition of the displaced atoms must be considered also. Further, before the implanted ions come to rest in the material they do not have the 
required energy for the displacement of target atoms. Therefore, the implantation species concentration peaks at a slightly larger depth than the ion-implantation induced damage.

It is apparent that the above considerations only refer to the initial damage situation during the passage of the ion into the material. However, in principle there could be complete recovery after the passage of each ion. The components in a collision cascade will include vacancies and interstitials, together with more complex arrangements of defects. It appears from computer simulations that the core of the cascade will be vacancy rich whereas interstitials will predominantly appear at the outer parts of the track. Nevertheless, this separation of vacancies and interstitials is insufficient to stabilize them and a very large percentage of the damage recovers on the timescale of nanoseconds. An accurate calculation of the final implantation damage depth distribution is further complicated by the change in the displacement energy during the ion implantation process. In a crystalline target, this displacement energy will decrease with increasing lattice disorder, i.e. it will decrease with lower atomic packing densities.

Figure 2-8 shows as an example the result of a Monte Carlo computer simulation of a $40 \mathrm{keV}$ proton implantation into silicon. The simulation shows an average of 12 target atom displacements by one incident ion.

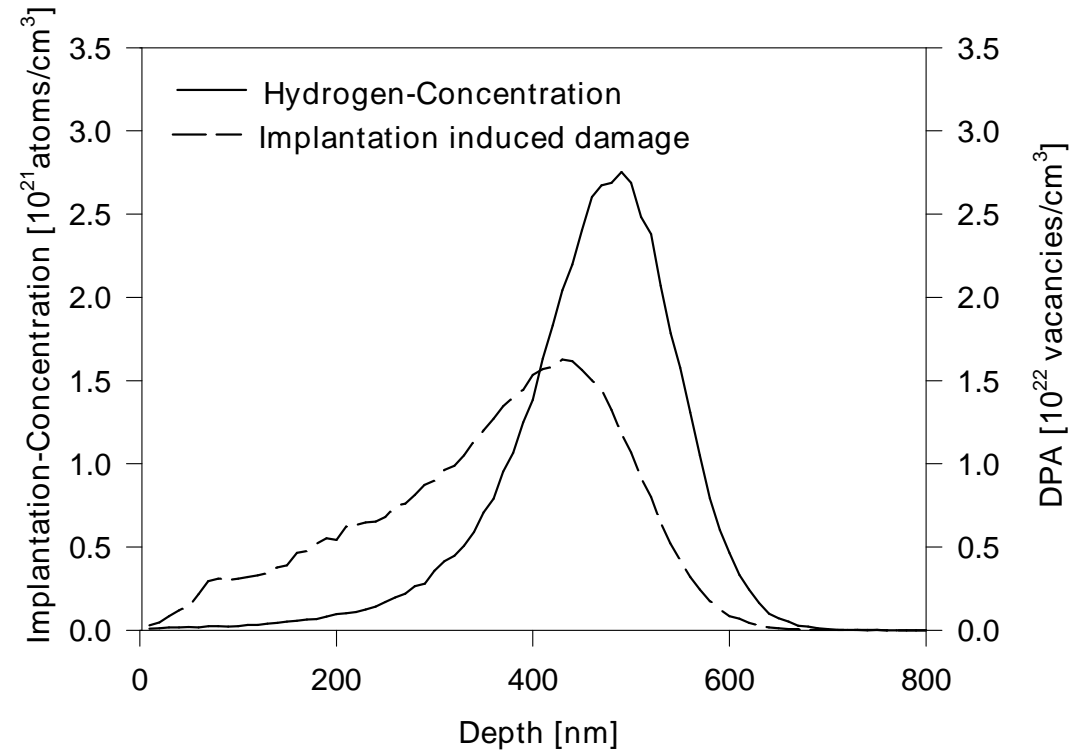

Figure 2-8: Monte Carlo computer code TRIM (Transport of Ions in Matter) [75] calculation of the hydrogen concentration and damage depth distribution for a $40 \mathrm{keV}$ proton implantation into silicon to a proton dose of $5 \times 10^{16} \mathrm{~cm}^{-2}$. 


\section{Experimental: Ion Implantation}

The sample preparation for all specimens used in this work included implantation of hydrogen, boron, and/or silicon ions. The energies of the implantation species ranged from 40 to $380 \mathrm{keV}$.

The ion implantations were carried out on a Varion/Extrion Division ion implanter model 200-DF4. The atomic implantation species are ionized, extracted under vacuum from the source, analyzed, accelerated to high energies, focused, electrically scanned and deflected towards the target. Figure 3-1 shows the systems diagram of the ion implanter.

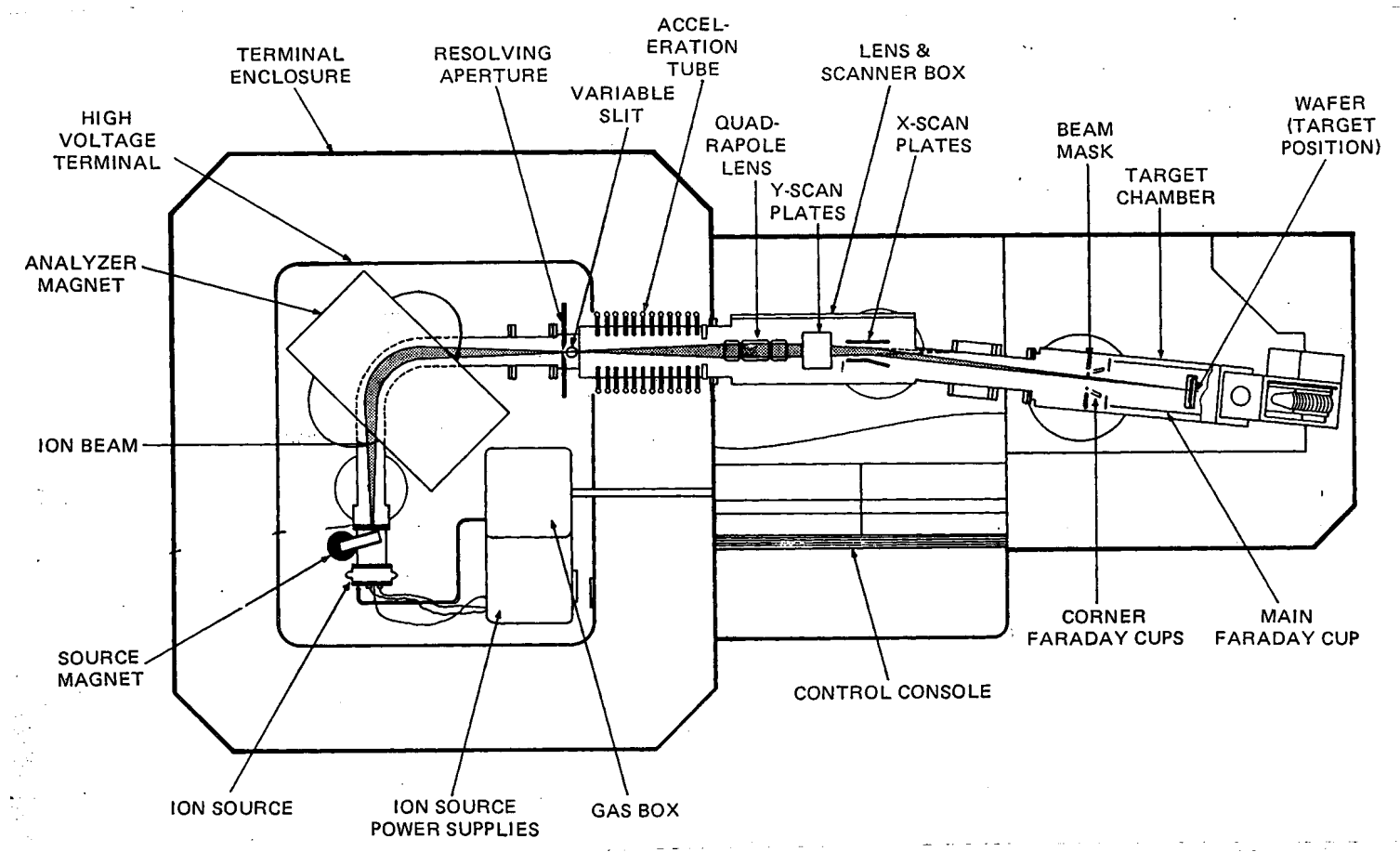

Figure 3-1: Systems diagram of the 200-DF4 ion implanter (image from [76]).

To produce a plasma, gas atoms are injected into an arc chamber of the discharge ion source. A negatively biased filament in the ion source is heated to emission temperature emitting electrons to the surrounding anode, the arc chamber wall. As the electrons 
collide with the gas molecules, a plasma of positive ions forms. The electron path is increased due to the weak axial magnetic field created by the source magnet. This causes more collisions between electrons and neutral gas molecules, creating a dense plasma of positive ions. To obtain a stable plasma, a gas mixture of argon and hydrogen was used for proton implantations. When boron or silicon ions were implanted, a boron or silicon powder was placed in the ion source, respectively. The gas mixture consisted of argon and carbon tetrachloride. Argon ions provided a stable plasma, whereas the carbon tetrachloride was required to obtain boron or silicon gas atoms for ionization.

The positive charged ions are extracted through the extraction electrode assembly. The extraction voltage is $32 \mathrm{kV}$ and hence the ion energy of single charged ions after the extraction is $32 \mathrm{keV}$. A mass analyzer magnet separates the ions according to their atomic masses to gain adequate ion purity. After the ions pass through the resolving aperture and the variable slits, the selected ion beam traverses through the acceleration tube, where it is accelerated to the implantation energy of up to $200 \mathrm{keV}$ for single charged ions. The quadrupol triplet lens, located in the beam line and past the grounded end of the acceleration tube, focuses the ion beam on the target (see figure 3-2): The electrostatic field, created by the lenses increases with increasing distance from the center of the ion beam and thus deviates ions more the further their distance is from the center of the beam. Consequently, in the horizontal direction, the ion beam gets less defocused when it traverses the first lens than it gets focused during its pass through the second lens. The horizontal defocusing effect of the third lens on the ion beam is negligible. Similarly, the quadrupol triplet lens system focuses the ion beam in vertical direction.
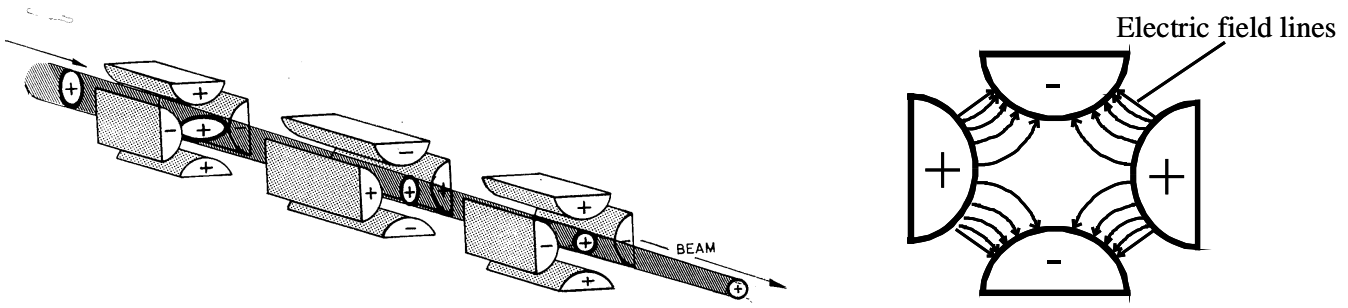

Figure 3-2: Drawing of the quadrupol triplet lens system (left image from [76]).

The focused ion beam passes through the beam scan system, consisting of two horizontal and vertical oriented plates, the so-called X and Y-plates. An electrostatic field created by the $\mathrm{X}$ and $\mathrm{Y}$ plates and the scan signal on those plates move the beam across the target surface area: An AC signal on the Y-plates scan the beam vertically at a frequency of $117 \mathrm{~Hz}$ over the target surface area. A DC offset voltage on the X-plates bends the beam $7^{\circ}$. Neutral particles formed in the acceleration tube and the beam line beyond the 
analyzer magnet are not affected by the CD offset and thus, are removed from the beam. Overlaid to the DC level on the X-plates is a scanning signal, which scans in the horizontal direction at a frequency of $102 \mathrm{~Hz}$. The triangular signal on the scanner plates scans the beam randomly across the wafer for uniform coverage (see figure 3-3).

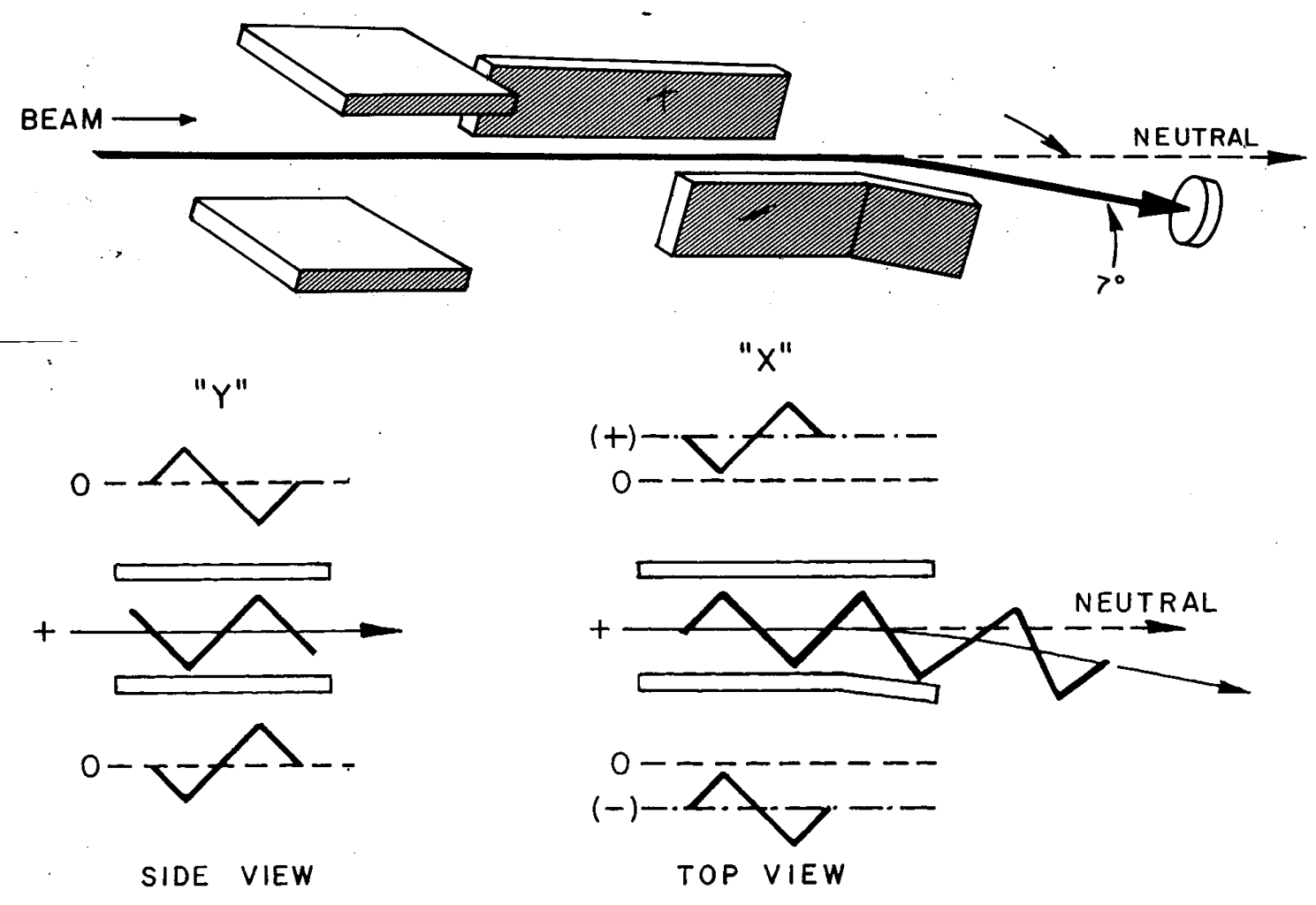

Figure 3-3: Schematic illustration of the scanning method (images from [76]).

The implantation dose is measured by the use of a Faraday system. The error in the implantation dose is $\leq 0.5 \%$. Four smaller Faraday cups measure the beam current at each corner of the scan pattern and are used to control the areal implantation dose uniformity. The areal implantation dose uniformity was typically $\leq 0.75 \%$.

The silicon wafers were attached to the sample stage by gluing them on with silver paint. The silver paint provided good thermal contact to the sample stage so that the heat generated in the implantation zone by the ion irradiation could be well dissipated. The samples were either implanted at room temperature or cooled down to a temperature of $77 \mathrm{~K}$ by pouring liquid nitrogen into the hollow sample stage. The implantation current was kept under $5 \mu \mathrm{A} / \mathrm{cm}^{2}$ to avoid uncontrolled ion beam heating of the silicon sample. 


\section{Experimental: Analyses Techniques}

For the present work, ion beam analyses such as Rutherford Backscattering Spectrometry (RBS), RBS in channeling mode, Transmission Channeling, and Elastic Recoil Detection (ERD) analysis were employed to obtain accurate information about the depth distribution of the implantation species and the ion irradiation induced crystal damage. To gain more precise knowledge of the depth profile of the implantation species, Secondary Ion Mass Spectroscopy (SIMS) was applied in addition to ERD.

The surface topography was quantitative determined by Atomic Force Microscopy (AFM) and scanning electron microscopy (SEM). Transmission Electron Microscopy (TEM) provided access to information about the microstructure of the ionimplanted specimen down to molecular dimensions. The ion-implantation induced compressive stress in the subsurface region of the silicon wafers was deduced from wafer curvature measurement with a laser scanning setup. The following sections give a detailed description of all analysis methods mentioned above.

\subsection{Composition Analysis}

\subsubsection{Rutherford Backscattering Spectrometry (RBS)}

Rutherford Backscattering Spectrometry (RBS) was used to determine the surface layer thickness of the ion-implanted and ion-cut samples as well as their homogeneity and stochiometry. RBS is based on the coulombic electrostatic repulsion of the nucleus, which causes the analyzing beam particles to backscatter after elastic collisions with target atoms.

The application of the technique to thin film analysis is illustrated in figure 4-1 for the case of a silicon sample, capped with a $\mathrm{SiO}_{2}$ layer and a very thin $\mathrm{Pd} \mathrm{Au}$ layer on top. The ions from the analyzing beam are directed towards a target with the energy $E_{0}$ and 
scatter elastically from target atoms with energies characteristic for the target atom masses, from which the ions are scattered. The analyzing ions also loose energy passing into and out of the target material. Since the energy $E_{0}$ of the analyzing beam is in the $\mathrm{MeV}$ regime, this energy loss is largely due to electronic interaction of the ion beam with the target (see also figure 2.3). Energy analysis of the backscattered ions by a solid state detector generates the backscattering spectrum displayed in the lower part of figure 4-1. The channel number is linearly related to the backscattered ion energy, $E_{l}$. Appearing in the spectrum are signals for each element present in the sample. The height of each signal reflects the concentration of the element in the target. The signal width is due to the energy loss, the analyzing ions undergo when they traverse the target. Therefore, the peak width represents the thickness of the layer in which the element is present.
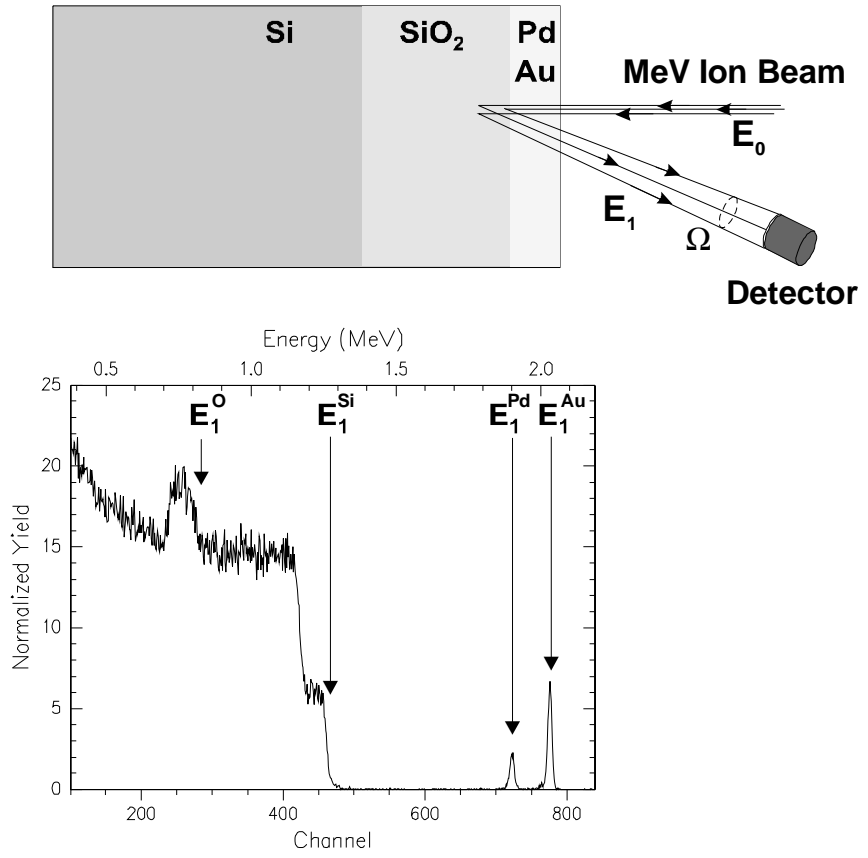

Figure 4-1: Rutherford backscattering spectroscopy: Experimental geometry (upper part). Rutherford backscattering spectrum (lower part)

The mass resolution depends on the kinetic energy transfer from the elastically scattered particles. The final energy $E_{l}$ of the analyzing particle of mass $M_{l}$ elastically scattered at an angle $\theta$ from a nucleus of mass $M_{2}$ at the sample surface is simply calculated using the conservation of momentum and energy:

$$
E_{1}=K\left(M_{2}, \theta\right) \cdot E_{0}=\left[\frac{\left(M_{2}^{2}-M_{1}^{2} \sin ^{2} \theta\right)^{1 / 2}+M_{2} \cos \theta}{M_{1}+M_{2}}\right] \cdot E_{0}
$$

$E_{0}$ is the initial energy and the scattering angle in the laboratory system. $K$ denotes the kinematic factor for the elastic scattering process. For any combination of projectile and 
target mass, the kinematic factor has its lowest value at $\theta=180^{\circ}$. Hence, in the experimental setup used here, the detector was placed at the largest possible angle $\theta$ of $165^{\circ}$ the setup geometry allowed for. For a fixed angle $\theta$, the energy separation, $\Delta E_{1}$, of beam particles scattered by target particles of mass difference $\Delta M_{2}$ is

$$
\Delta E_{1}=E_{0} \cdot\left(\frac{d K}{d M_{2}}\right) \cdot \Delta M_{2}
$$

If $\Delta E_{1}$ is set equal to $\delta E$, the minimum energy separation that can be experimentally resolved, and the mass resolution $\delta M_{2}$ is determined to be

$$
\delta M_{2}=\frac{\delta E}{E_{0}\left(\frac{d K}{d M_{2}}\right)}
$$

The mass resolution at the sample surface is usually determined primarily by the detector resolution, while deeper in the layer the straggling dominates. In the detector used in this work the energy width per channel and hence the energy resolution was about $2.5 \mathrm{keV}$.

The yield of backscattered particles, i.e. the probability for backscattering, is determined by the cross-section $\sigma_{i}(E, \theta)$ of the scattering process. With the knowledge of the measured or calculated cross section $\sigma_{i}(E, \theta)$ the areal density, $(N t)_{i}$, in atoms per unit area for the $i$ th element, can be determined from the values of the detector angle $\Omega$, the integrated peak count $A_{i}$, for $\mathrm{Q}$ incident ions:

$$
(N t)_{i}=\frac{A_{i} \cos \theta_{1}}{Q \Omega \sigma_{i}(E, \theta)}
$$

$N$ is the atomic density (atoms per unit volume) and $t$ the thickness of the analyzed film. If the scattering is Rutherford, i.e. pure Coulomb scattering, $\sigma_{i}(E, \theta)$ in the laboratory system can be calculated from

$$
\sigma(E, \theta)=\left(\frac{Z_{1} Z_{2} e^{2}}{4 E}\right)^{2} \cdot \frac{4\left[\left(M_{2}^{2}-M_{1}^{2} \sin ^{2} \theta\right)^{1 / 2}+M_{2} \cos \theta\right]^{2}}{M_{2} \sin ^{4} \theta\left(M_{2}^{2}-M_{1}^{2} \sin ^{2} \theta\right)^{1 / 2}}
$$

The cross-section is proportional to $Z_{2}^{2}$ which means that for any given projectile, heavy atoms are more efficiently scattered than light ones. The proportionality to $E^{-2}$ leads to an increased scattering yield with decreasing ion energy. 
The real cross sections deviate from the Rutherford cross section at both high and low energies for all projectile-target atom pairs. [77] At low energy the deviation from $\sigma_{R}$ is due to the partial screening of the nuclear charges by the electron shells surrounding both nuclei. At high energies the deviation from $\sigma_{R}$ is caused by the presence of shortrange nuclear forces.

The analyzing ions, which are backscattered from atoms located in deeper parts of the target, undergo inelastic scattering with the electron cloud of the target atoms and hence lose energy continuously on their way into and out of the material. The radii of atomic nuclei are so small compared with atomic dimensions that nuclear scattering is rare compared to interactions with electrons. The final energy of the emerging particle becomes

$$
E_{1}=K\left(E_{0}-\Delta E_{\text {in }}\right)-\Delta E_{\text {out }}
$$

The stopping power of a material for a particular ion is defined as the energy loss per distance traveled in the material, denoted as $d E / d x$. The stopping powers depend on the target material, the ion species and also the ion energy. Another quantity, the stopping cross section $\varepsilon$ is defined as the energy loss / areal density of the target material (energy loss $/$ atoms $/ \mathrm{cm}^{2}$ ). The relation between the stopping cross section and the stopping power is given as

$$
\frac{d E}{d x}=N \varepsilon
$$

where $\mathrm{N}$ is the atomic density (atoms/volume unit). To convert the energy axis of the RBS spectrum into a depth scale requires the solving of the integral

$$
x=\int_{E_{1}}^{E_{0}}(d E / d x)^{-1} d E
$$

where $\mathrm{x}$ is the target depth of the backscattering event. The Bragg rule is used to calculate stopping powers of compound targets. The Bragg rule assumes that the interaction between the incident ions and the target atoms do not depend on the environment. Phase effects and chemical bonds between the target atoms are disregarded and the assumption is made that each target atom acts independently in the energy loss process. These assumptions lead to the approximation

$$
\frac{d E}{d x}=N \sum c_{i} \varepsilon_{i}
$$

where $c_{i}$ and $\varepsilon_{i}$ are the concentration and stopping cross section for element $i$, respectively. 
A quantitative knowledge of the stopping power or the stopping cross section is a key element in determining the thickness of the target layers. For this work, accuracy in the layer depth measurements turned out to be crucial for the exploration of the mechanisms behind the Ion-Cut process. Therefore, a large emphasis was placed on the accuracy of the stopping power data used. A considerable number of experimental studies on the ion energy loss have been conducted. These data on ion stopping ranges are collected in a number of compilations [78-80]. A range table from Ziegler et al. is commonly used and also implemented in the Monte-Carlo simulation program TRIM [75]. For the ion-target system in this work, i. e. $\alpha$-particles in silicon, an extensive literature search was conducted. The recently published experimentally determined stopping cross-section by Konac et al. [81] was found, to the best knowledge of the author, to be the most accurate (see figure 4-2). The inaccuracy of these stopping power values is cited to be $1-2 \%$.

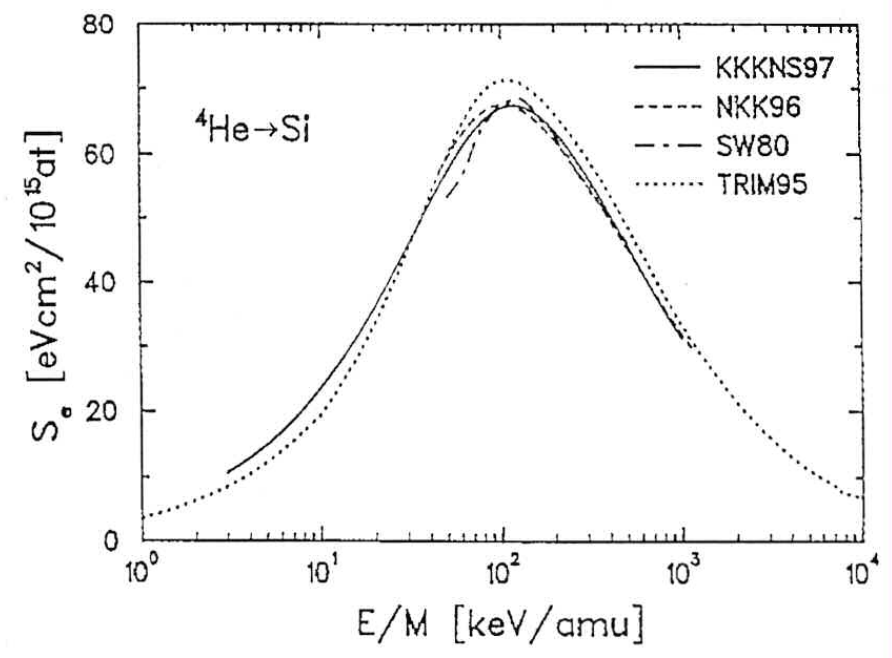

Figure 4-2: Electronic stopping cross-sections for helium ions in silicon vs. energy/atomic mass. (KKKNS97 $=$ Konac et al. [81], (the stopping cross-section used for this work), NKK96 $=$ Niemann et al. [82], SW80 = Santry and Werner [83], TRIM95 = Ziegler et al. [75])

The accelerator used for RBS measurements was a National Electrostatics Corporation $3 \mathrm{MV}$ tandem accelerator located at the Ion beam Materials Laboratory at Los Alamos National Laboratory, New Mexico, USA. The RBS spectra were obtained using a ${ }^{4} \mathrm{He}^{+}$ beam with energies ranging from $2.0 \mathrm{MeV}$ to $3.036 \mathrm{MeV}$. The dimensions of the analyzing beam cross-section were approximately $1 \mathrm{~mm} \times 2 \mathrm{~mm}$. The beam current ranged from 10 to $45 \mathrm{nA}$. The backscattered $\alpha$-particles were registered by means of a silicon surface detector, positioned at an angle of $165^{\circ}$ relative to the analyzing beam 
subtending a solid angle $\Omega$ of 2.34 mstr. The spectra were analyzed employing the computer code RUMP [84]. For the conversion of the energy axis into a depth scale, the stopping power data of ${ }^{4} \mathrm{He}^{2+}$ ions in Si from Konac et al. [81] were implemented.

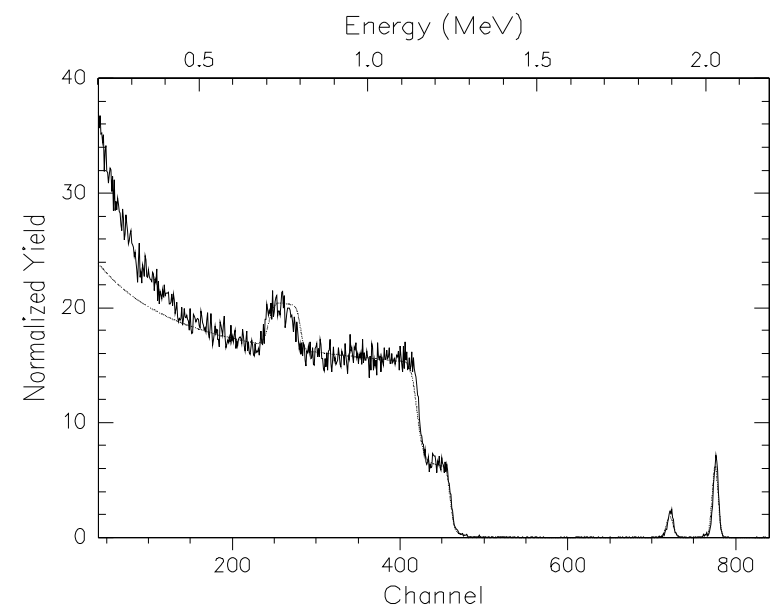

Figure 4-3: RBS spectrum of the sample shown in figure 4-1. The simulation of the spectrum, calculated with the computer code RUMP [84] is overlaid.

Figure 4-3 shows as an example the RBS spectrum of sample shown in figure 4-1, overlaid with the computer simulation. The simulation reveals that the sample consists of $1 \mathrm{~nm}$ thick $\mathrm{Au} / \mathrm{Pd}$ layer on top, followed by a $\mathrm{SiO}_{2}$ layer with a thickness of $310 \mathrm{~nm}$. The deviation of the simulation from the RBS spectrum at lower channel number is due to multiple scattering, a phenomenon mainly occurring at lower energies and not accounted for in the RUMP calculations. 


\subsubsection{Elastic Recoil Detection (ERD) Analysis}

Elastic recoil detection (ERD) analysis is an ion beam technique whereby quantitative information about sample composition can be obtained by detecting light target nuclei recoiling from heavier bombarding ions. In this work ERD was employed to obtain implanted hydrogen depth distributions using a ${ }^{4} \mathrm{He}^{+}$beam of several $\mathrm{MeV}$ energy. The ERD experiments were carried out in reflection geometry, in which the incident ion beam strikes the target at a grazing angle, and recoiled and scattered particles emerge from the target's front surface. Figure 4-4 shows schematically the setup of the experiment.

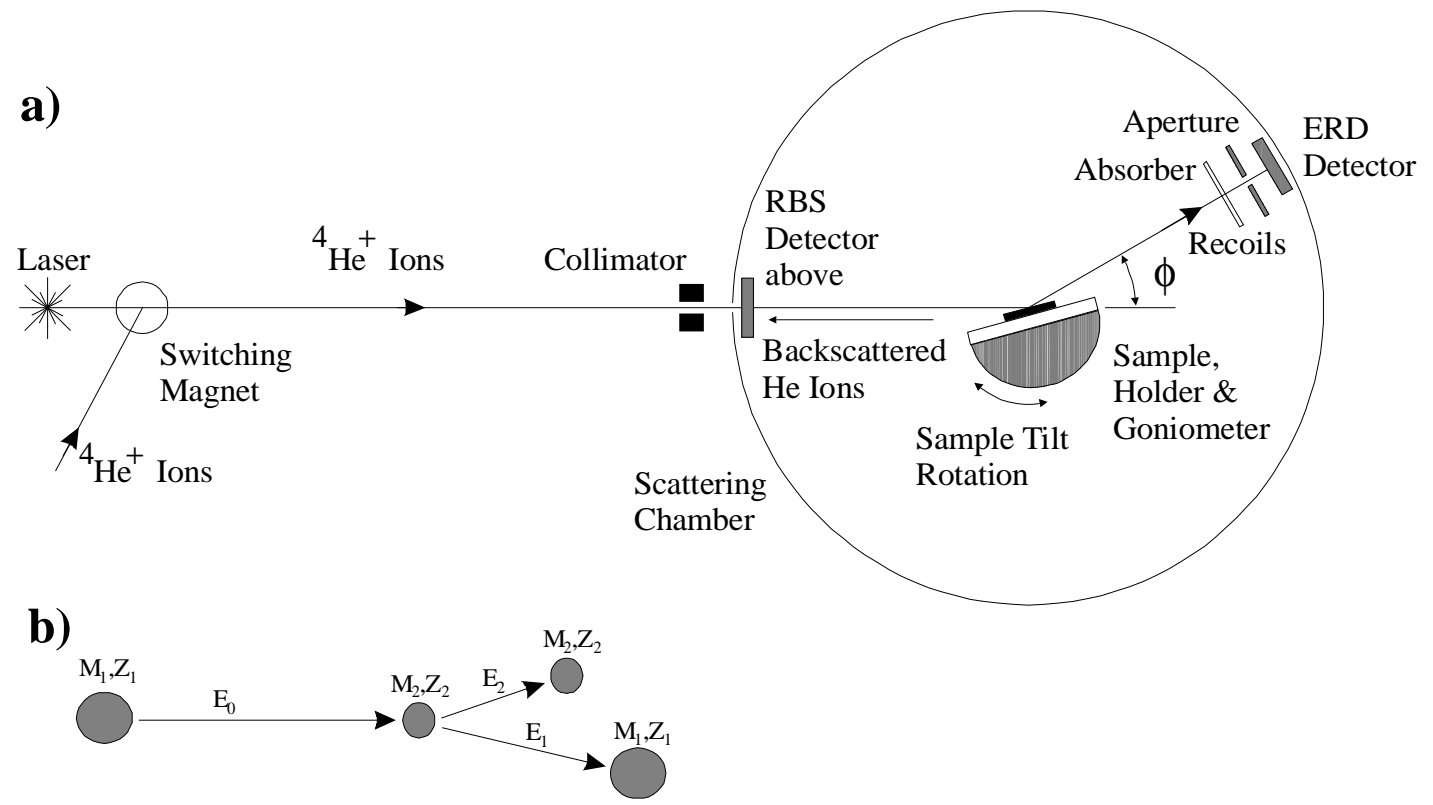

Figure 4-4: Schematics of (a) the experimental arrangement and (b) the elastic scattering between the target $\mathrm{M}_{2}$ and the beam particle $\mathrm{M}_{1}$ in the laboratory frame.

The recoil angle $\phi$ lies between the path of the recoiled particle to the detector and the continuation of the path of the incident beam. In the present arrangement $\phi$ was $29.5^{\circ}$. Calibrating the tilt angle was performed with a $\mathrm{HeNe}$ laser beam coincident with the path taken by the incident particle beam and directed into the scattering chamber. With the laser alignment of the sample tilt angle a reproducible accuracy of $0.04^{\circ}$ was achieved. To avoid detection of forward-scattered $\alpha$-particles, a Mylar foil, 12 to $24 \mu \mathrm{m}$ in thickness (dependent on the ion beam energy), was positioned between the sample and the hydrogen detector. 
The conservation of energy and momentum give the energy of the recoiled atom

$$
E_{2}=E_{0} \frac{4 M_{1} M_{2}}{\left(M_{1}+M_{2}\right)^{2}} \cos ^{2} \phi
$$

where $E_{0}$ is the energy of the ion beam and $M_{1}$ and $M_{2}$ are the atomic masses of the incident ions and the target atoms, respectively. $\phi$ is the angle between the incident beam and the direction of the recoiled atoms. The energy of the incident ion after the elastic collision is

$$
E_{1}=E_{0}\left(\frac{\sqrt{M_{2}^{2}-M_{1}^{2} \sin ^{2} \phi}+M_{1} \cos \phi}{M_{1}+M_{2}}\right)^{2}
$$

\section{Depth profiling}

The incoming ions from the analyzing beam and the forward recoiled hydrogen atoms loose energy as they traverse the sample. As in RBS, from this energy loss, the depth distribution of the hydrogen atoms can be deduced. Correct depth profiling of hydrogen in crystalline silicon from elastic recoil detection analysis by conventional ERD simulation inhibits possible errors like inaccuracies in the geometric arrangement (scattering angle, eucentric position, etc. [85]), and errors in the stopping power values for the forward scattered hydrogen in the target.

However, accurate depth profiling of the implantation hydrogen was essential for the investigation of the Ion-Cut process. Therefore, a technique was developed, which converts the channel difference between surface and bulk signals directly to depth. This channel-to-depth conversion method uses fitting data from ion beam analysis: RBS and ERD analysis were performed simultaneously on specially prepared calibration standards; channel to depth conversion was determined from the forward scattering data and computer simulation of the backscattering data [86].

Ten depth calibration standards were prepared on c-Si substrates by sputter depositing a zirconium + hydrogen $(\mathrm{Zr}+\mathrm{H})$ layer followed by an overlayer of amorphous silicon (a-Si) of different thickness for each standard (see figure 4-5). The zirconium acts as a getter for the hydrogen. 


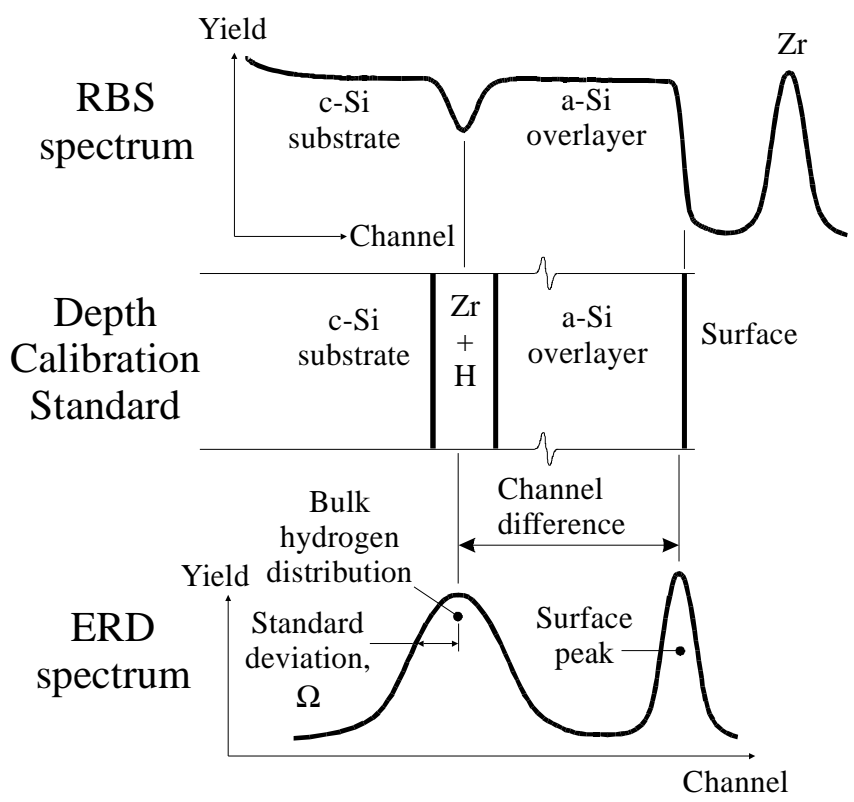

Figure 4-5: Schematic cross section of a typical depth calibration standard, the corresponding ERD spectrum (lower part) and RBS spectrum (upper part).

Secondary ion mass spectroscopy (SIMS) (see chapter 4.1.3) analysis revealed that the centroid of the hydrogen distribution was coincident with that of the zirconium marker at the midpoint of the $\mathrm{Zr}+\mathrm{H}$ layer, and that the signal from the hydrogen contained in the zirconium layer was an order of magnitude greater than the nearly constant background signal in the a- and c-Si regions

ERD and RBS measurements on these standards were performed simultaneously. To ensure that the experimental observations were independent of energy variations, all ERD/RBS experiments were performed at the ${ }^{16} \mathrm{O}(\alpha, \alpha){ }^{16} \mathrm{O}$ resonance energy of $3.036 \mathrm{MeV}$, which has a full width at half maximum of $8 \mathrm{keV}$ [87].

By simulating the RBS spectra of the depth calibration standards, the areal densities of the a-Si overlayer (different for each standard) and $\mathrm{Zr}+\mathrm{H}$ layer were determined, thus locating the centroid of the buried zirconium marker relative to the surface. Locating the centroid of the buried zirconium distribution also located the centroid of the bulk hydrogen distribution, as the two were shown to be coincident by the SIMS analysis. Figure 4-6 plots a RBS and ERD spectrum obtained simultaneously from one of the depth calibration standards. 


\section{a)}

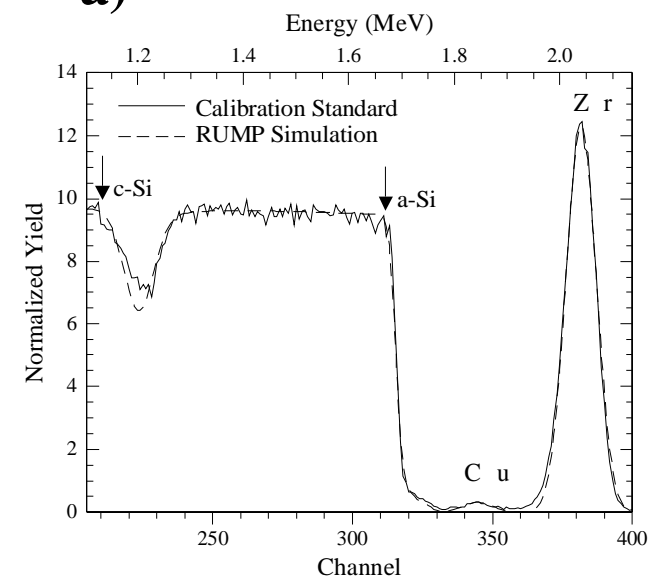

b)

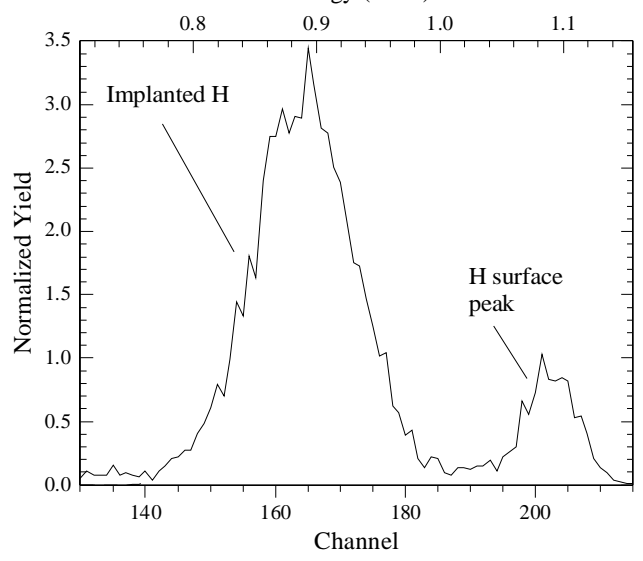

Figure 4-6: Representative RBS (a) and ERD (b) spectrum and RBS simulation of the depth calibration standards at a nominal tilt angle of $75.25^{\circ}$. The two spectra were obtained simultaneously.

The channel difference between the hydrogen surface and bulk centroids (ERD spectrum) was compared to the effective overlayer areal density (simulation of RBS spectrum) for each depth calibration standard. The data from two runs are plotted in figure 4-7 along with the power law fit $f(x)$, a non-linear least squares fit of the average data values given by

$$
f(x)=a \cdot x^{b}
$$

where $x$ is the channel difference between the hydrogen surface and bulk centroids and the values of $a$ and $b$ were determined by the fit. The lengths of the error bars on the channel difference data are less than the size of the symbols.

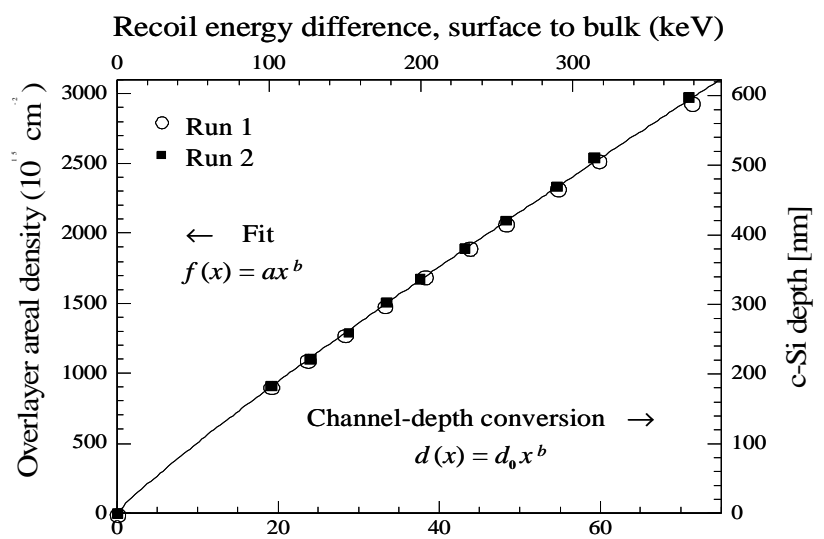

Channel difference, surface to bulk centroids
Figure 4-7: Data of the effective overlayer areal density plotted against the difference between surface and bulk centroids for 2 runs of 10 depth calibration standards. The length of the error bars for the channel difference data is less than the size of the symbols. The power law fit of the average of the data is shown. The detected recoil energy difference and c-Si depth axes are also included. (The graph is from [86])

The major advantage of the described depth profiling method is the use of the $\mathrm{Zr}$ signal in the RBS spectrum for the depth calibration of ERD spectra, eliminating possible errors in conventional ERD simulations due to inaccuracies of the stopping power values for the 
forward scattered hydrogen in the target. Furthermore, in simulating ERD spectra it appears that there is no unambiguous way of determining the surface hydrogen distribution. ERD spectra do not provide the information whether the hydrogen surface peak is due to hydrogen residing on top of the wafer surface or hydrogen absorbed into the subsurface region of the sample. The two different methods of simulating the surface peak lead to simulated hydrogen depth distributions, which slightly differ from each other. However, the depth profiling method described here offers a distinct advantage in that it is independent of actual or simulated surface hydrogen distributions.

If the a-silicon overlayer exceeded the thickness of $600 \mathrm{~nm}$, an overlap in the RBS spectrum of the $\mathrm{Zr}$ signal with the Si-signal occurs, preventing an accurate analysis of the RBS spectrum. Therefore, the channel-to-depth conversion was made for depths up to $600 \mathrm{~nm}$ only.

When the hydrogen implantation range was larger than the depths covered in the channel-to-depth conversion, or different analyzing beam energies were chosen the ERD data were analyzed using the computer code RUMP [84]. For the incoming He ion beam, as in the RBS analysis, the stopping cross sections from Konac et al. [81] were implemented in the computer program. The stopping power of the forward recoiled hydrogen atoms was also taken from the work of Konac et al. (see figure 4-8) and implemented in the simulations, since their accuracy was shown to be 1-2\% [88]. For the evaluation of the total hydrogen content literature values of energy dependent cross sections for helium-hydrogen collisions, which apply to our experimental setup (scattering angle) were implemented in the computer program. [89]

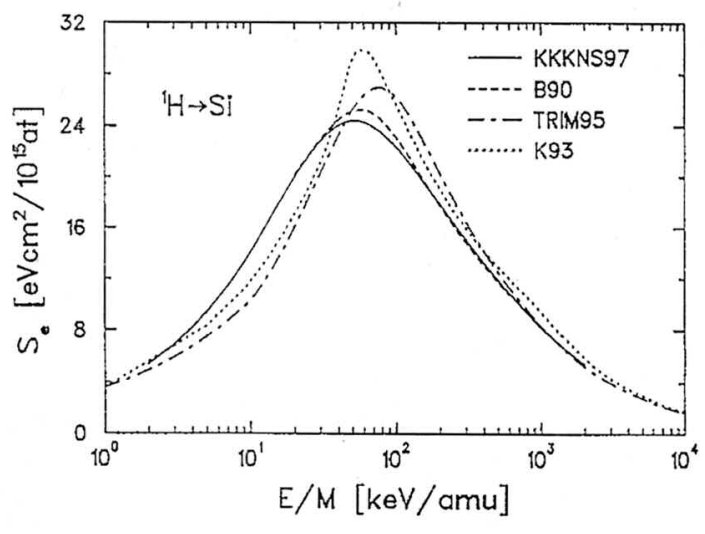

Figure 4-8: Electronic stopping cross-sections for protons in silicon vs. energy/atomic mass. (KKKNS97 = [81], (the stopping cross-section used for this work), B90= Bauer.[90], TRIM95 = Ziegler et al.[75]), K93 = Kaneko [91]. 


\section{Energy Spread}

Energy spread is inherent in ERD analysis. The energy spread broadens the measured energy distributions and impairs the depth resolution of the measured hydrogen depth distribution. Similar to the categorization by Tirira et al. [92] and Szilagyi [93] groups the components of energy spread in ion beam analysis into two categories: First are the extrinsic components, pertinent to the experimental setup, of 1) energy spread of the incident beam, 2) angular spread of the incident beam, 3) geometric spread due to the finite extents of the incident beam and detector aperture, 4) detector resolution, and 5) energy spread from absorber foils, if any, required to block scattered incident ions from flooding the ERDA detector. The second category is comprised of the intrinsic components, pertinent to the sample, of 6) energy straggle due to the statistical fluctuation in the number of collision processes, and 7) multiple scattering attributed to the collisions between the incident particles and the host nuclei.

The channel-depth conversion described above does not correct for energy spread, the unwanted broadening in the energy of the spectra, which can lead to errors in depth profiling. Therefore, a technique was developed, that corrects for energy spread in elastic recoil detection analysis spectra, the energy spread correction. [94] The depth profiling method described here corrects for both extrinsic and intrinsic energy spread components.

Following standard nomenclature, the variance of a distribution, $\Omega^{2}$, is defined to be the square of the standard deviation, $\Omega$, which is shown in the ERD schematic in figure 4-5. In constructing the model for the energy spread correction the assumption was made that the total variance of an ERD spectrum, $\Omega^{2}{ }_{T O T A L}$, can be expressed as the sum of the individual components [95]. Then

$$
\Omega_{\text {TOTAL }}^{2}=\Omega_{E S}^{2}+\Omega_{\text {ACTUAL }}^{2}
$$

expresses the total variance as the sum of the energy spread components, $\Omega^{2}$ ES and the actual variance, $\Omega^{2}{ }_{\text {ACTUAL }}$, corresponding to the distribution of target hydrogen atoms in the sample. Because the components of the energy spread can be determined experimentally, equation (4-13) gives a means for calculating the actual variance:

$$
\Omega_{A C T U A L}^{2}=\Omega_{\text {TOTAL }}^{2}-\Omega_{E S}^{2}
$$

The energy spread is determined by ERD analysis of the depth calibration standards shown above (see figure 4-5). The $\mathrm{Zr}+\mathrm{H}$ layer in the standards are very thin, so that their thickness can be used to compute the (ideal) actual energy difference between hydrogen recoils from the front and back of the $\mathrm{Zr}+\mathrm{H}$ layer, $\sim 6 \mathrm{keV}$ (about half the ERDA system resolution). The half of this value, $3 \mathrm{keV}$, can be taken as the actual standard deviation of 
the depth calibration standards, which is much smaller than the standard deviation of their ERD spectra. Squaring the standard deviations to obtain the variances will only magnify the disparity, so the value of the actual variance will be negligible compared to the total variance of a spectrum. Then, from equation 4-13 the standard deviation of the energy spread, $\Omega^{(i)}$ ES, can be approximated at a depth corresponding to the overlayer areal density of the $i^{\text {th }}$ depth calibration standard as

$$
\Omega_{E S}^{(i)} \approx \Omega_{S T A N D A R D}^{(i)}
$$

where $\Omega^{(\mathrm{i})}$ STANDARD is the standard deviation of the spectrum of the $i^{\text {th }}$ depth calibration standard. By equation 4-15, the $\mathrm{Zr}+\mathrm{H}$ layer is approximated to be infinitesimally thin, which implies that the energy spread being measured consists of both the extrinsic component pertinent to the experimental setup, and the intrinsic component pertinent to the silicon host.

The energy spread in equation 4-15 is a function of the areal density of the a-Si overlayers; the energy spread increases with increasing overlay areal density. Equivalently, the energy spread can be expressed as the spread in channels vs. the channel difference between the surface peak and bulk centroids of the ERD spectra. Figure 4-9 shows this relationship in terms of channels for a Mylar foil thickness of 14.5 $\mu \mathrm{m}$. Expressing the energy spread in this way allows the analysis for the energy spread correction to be performed in terms of channels.

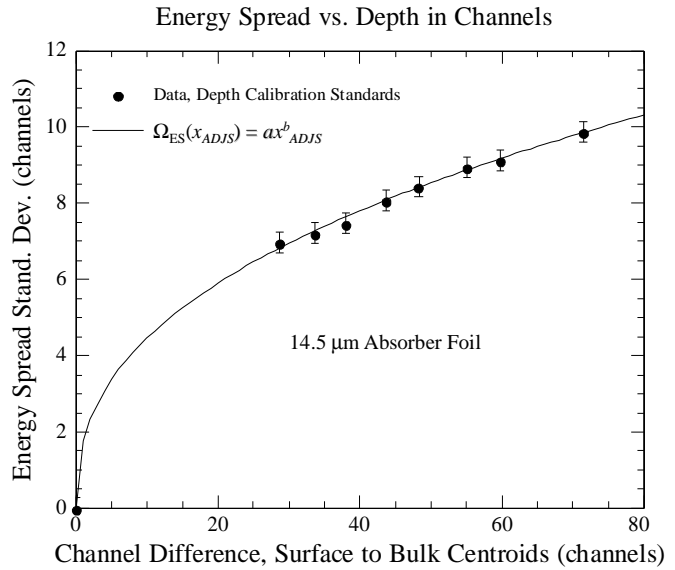

Figure 4-9: Data of the energy spread standard deviation vs. the channel difference from surface contamination peak to bulk distribution centroids of the ERDA spectra of the depth calibration standards. Also plotted is the fit to the data. (Figure from [94])

The fit line through the data is given by

$$
\Omega_{\mathrm{ES}}\left(x_{\text {Adjusted }}\right)=a x_{\text {Adjusted }}^{b}
$$

where $x_{\text {Adjusted }}$ is the channel difference from the surface peak centroid to a point in the bulk, and $a$ and $b$ are parameters determined by a least squares regression analysis. 
Equation 4-16 is an analytical expression for the energy spread as a function of $x_{\text {Adjusted }}$. Substituting equation 4-16 into equation 4-14 gives an analytical expression for the actual variance:

$$
\Omega_{A C T U A L}^{2}\left(x_{A D J S}\right)=\Omega_{T O T A L}^{2}-\Omega_{E S}^{2}\left(x_{A D J S}\right)
$$

Finally, the energy spread correction to the ERD spectrum is performed by scaling the channel axis of the adjusted spectrum using

$$
x_{\text {CORRECTED }}=\left(x_{A D J S}-c\right) \frac{\Omega_{A C T U A L}\left(x_{A D J S}\right)}{\Omega_{\text {TOTAL }}}+c
$$

where $x_{\text {CORRECTED }}$ is the scaled ("corrected") channel point, $c$ is the centroid of the adjusted spectrum, and the ratio $\Omega_{A C T U A L}\left(x_{A D J S}\right) / \Omega_{\text {TOTAL }}$ is defined as the correction factor.

Figure 4-10 shows the steps in the energy spread correction for a silicon sample, implanted with $40 \mathrm{keV}$ protons to a dose of $5 \times 10^{16} \mathrm{~cm}^{-2}$.
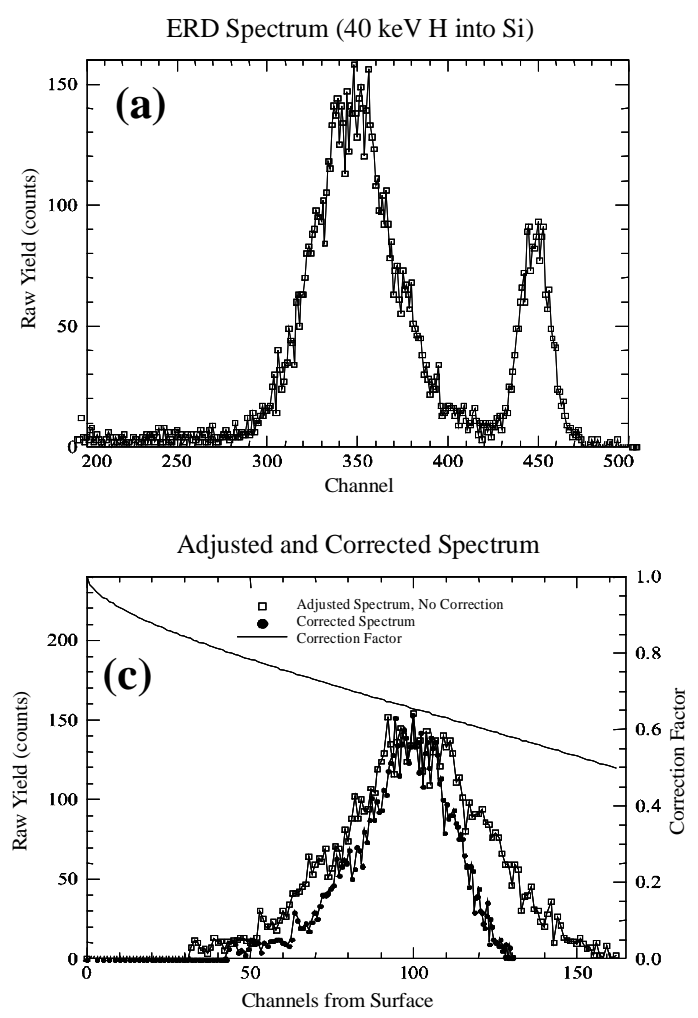
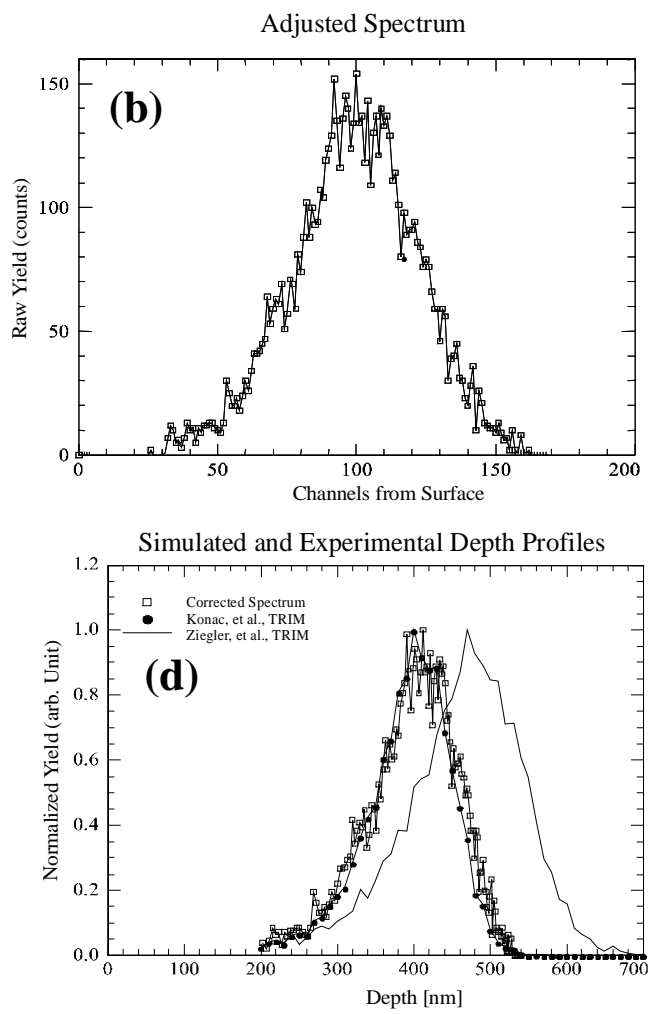

Figure 4-10: Analysis of a ERD spectrum obtained from a silicon wafer, implanted with $40 \mathrm{keV}$ protons to a dose of $5 \times 10^{16} \mathrm{~cm}^{-2}$. The ${ }^{4} \mathrm{He}^{+}$ion beam had an energy of $3.036 \mathrm{MeV}$. The raw spectrum (a) is converted to the adjusted spectrum (b), which plots the counts as a function of channel difference to the surface peak centroid. The adjusted spectrum is then corrected for the energy spread (c) and finally the xaxis is translated into the depth scale (d). To illustrate the accuracy of the chosen stopping powers for He and $\mathrm{H}$ in silicon, a TRIM simulation with the stopping powers from Konac et al. [81] (used in this work) and from Ziegler et al. [75] are overlaid. 
Fig. 4-10 d compares the depth profile obtained by the ERD analysis to TRIM simulations performed with the TRIM computer code [75] using either the stopping power data from Konac et al. [81] or from Ziegler et al. [75]. The results suggest that the stopping data used in this work are very accurate: The analysis of the ERD spectrum uses He stopping power in the $\mathrm{MeV}$ range, whereas the TRIM simulation uses $\mathrm{H}$ stopping power in the $\mathrm{keV}$ regime. However, the hydrogen depth obtained by the ERD analysis overlap perfectly with the one obtained by TRIM simulation.

\subsubsection{Secondary Ion Mass Spectroscopy (SIMS)}

Secondary Ion Mass Spectroscopy (SIMS) is an analysis method, which provides information about the depth distribution of implantation species of very low concentration. This chapter only gives a short introduction about the analysis method. For further information about SIMS, the reader is referred to literature. [96-98] In SIMS a primary ion beam with typical energies between 0.5 and $20 \mathrm{keV}$ and a constant current bombards the sample. The primary ion beam usually consists of $\mathrm{O}^{2+}, \mathrm{Cs}^{+}, \mathrm{Ar}^{+}$, or $\mathrm{Ga}^{+}$ ions. The bombardment with energetic primary ions leads to sputtering of neutral and ionized target atoms. These so-called "secondary ions" are separated in mass by a quadrupol mass spectrograph and subsequently collected by a electron multiplier detector. Figure 4-11 shows schematically the SIMS measurement setup.

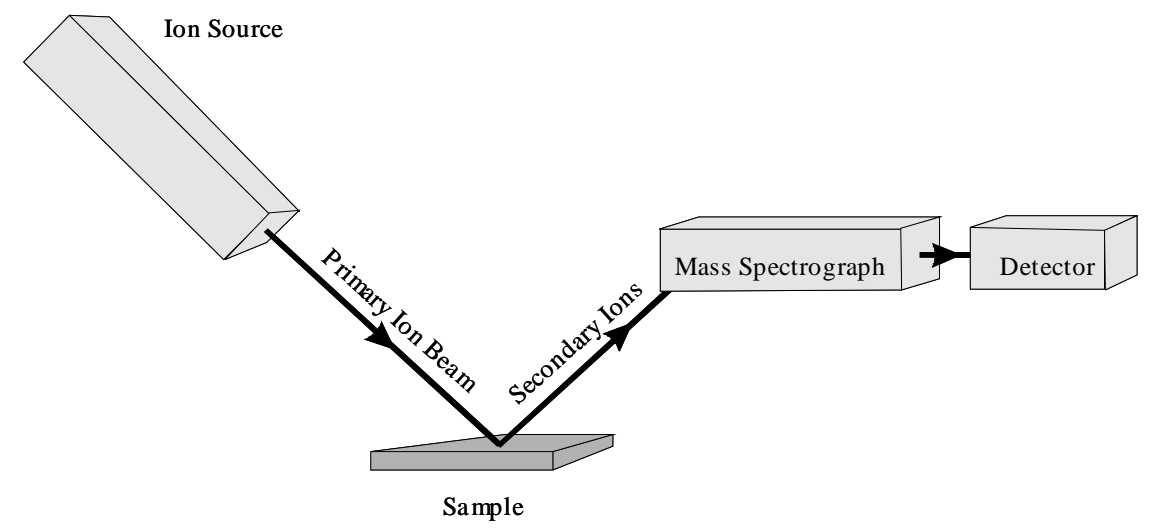

Figure 4-11: Schematic illustration of the SIMS measurement setup. 
The sputtering due to the bombardment of the target by the primary ion beam leads to the development of a crater in the target. The mass spectrograph monitors the amount of secondary ions detected per second as a function of time for each element of interest. Knowing the primary ion beam current and the sputter rate the conversion of the time scale into a depth scale can be calculated. In this work, the conversion of the time scale into a depth scale was accomplished by measuring the crater depth after the SIMS measurement with a profilometer.

For the interpretation of the obtained depth profiles it has to be taken into account, that the sputter rate is dependent on the amount of dopant in the material and the crystal quality of the semiconductor (here silicon). Since ion irradiation of the sample prior to the SIMS measurement led to a implant species concentration of up to $5 \%$ and induced implantation damage in the sample, the linear conversion from the time axis into a depth scales causes some artifacts in the depth distribution. Furthermore, it has to be considered, that secondary ions from the crater rim could condense on the sample surface. Therefore, the primary ion beam was scanned over a large area of $250 \mu \mathrm{m} \times 250 \mu \mathrm{m}$, but only the SIMS signal of the atoms emitted from the center of the crater (circle with diameter of $60 \mu \mathrm{m}$ ), were detected.

Despite the inaccuracies of SIMS measurements in terms of the linear conversion from the time scale into a depth scale, this analysis method provides precise data of the shape of the implantation profile. For the analysis of the hydrogen implantation depth distribution, ERD was used to determine the actual depth and the amount of the implanted hydrogen, whereas SIMS provided precise information about the shape of the H-depth distribution. SIMS was also applied to monitor the hydrogen depth distribution after the Ion-Cut. After ion-cutting the implanted hydrogen resides in the subsurface region and hence the energy straggling in the ERD spectra made it impossible to distinguish between the H-implant signal, and the signal representing surface contaminations. However, SIMS was able to distinguish between the signal from the hydrogen surface contamination and the signal from the hydrogen implant.

SIMS also allows the measurement of the low dose boron implantation profile in the $\mathrm{B}+\mathrm{H}$ co-implanted samples. The boron depth distributions could not be monitored by RBS because of the low atomic mass and the low implantation dose: The low atomic mass of boron compared to silicon leads to an overlap of the boron signal with the silicon signal and therefore, the signal of the low amount of the implanted boron could not be distinguished from the silicon signal.

The SIMS measurements were performed using a CAMECA IMS 3F spectrometer operated with a primary $6.2 \mathrm{keV} \mathrm{O}_{2}{ }^{+}$sputter beam. The primary beam 
current was $0.4 \mu \mathrm{A}$. To calibrate the depths scale of the obtained SIMS data the crater depths were measured with a "Alpha-Step 500" surface profiler from Tencor Instruments with a profiler tip of $2 \mu \mathrm{m}$ in diameter. The depth resolution was $1 \mathrm{~nm}$. Within the area from which the SIMS signal of the emitted secondary ions was collected (circle with 60 $\mu \mathrm{m}$ in diameter) the crater depth varied only over about $5 \mathrm{~nm}$. When $\mathrm{B}+\mathrm{H}$ co-implanted silicon samples were analyzed, the hydrogen and boron profiles were recorded simultaneously. Figure 4-12 shows the SIMS spectra of a $\mathrm{B}+\mathrm{H}$ co-implanted silicon wafer.

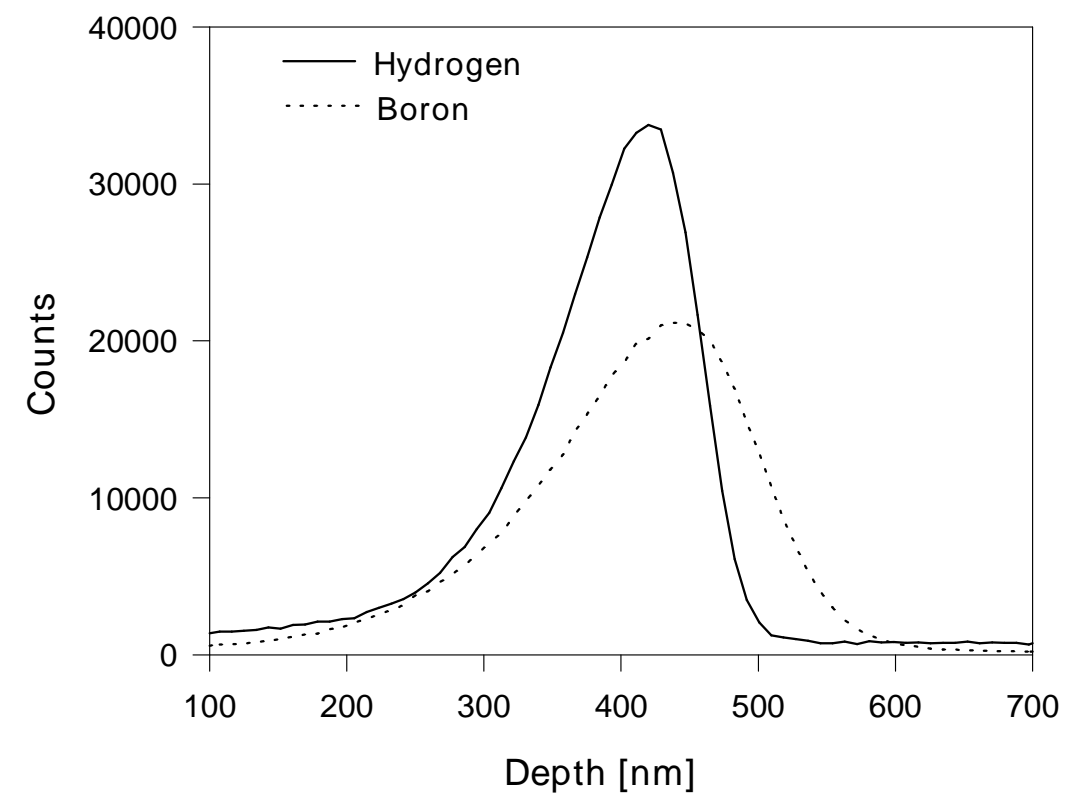

Figure 4-12: SIMS spectra of a silicon sample, implanted with $147 \mathrm{keV}^{11} \mathrm{~B}$ to a dose of $1 \times 10^{15} \mathrm{~cm}^{-2}$ and $40 \mathrm{keV}$ protons to a dose of $2.5 \times 10^{16} \mathrm{~cm}^{-2}$. The boron count rate was higher than the hydrogen count rate, therefore the boron signal appears to be quite large compared to the hydrogen signal. 


\subsection{Ion Beams for Crystal Damage Analysis}

\subsubsection{RBS Channeling}

RBS in channeling mode was employed to gain accurate information of the depth and amount of damage, induced by the ion-irradiation of silicon. This method also allowed the examination of the evolution of the implantation damage profile during heat treatments of the samples. Furthermore, RBS Channeling provided knowledge of the damage depth distribution after ion-cutting.

When high energetic ions are directed towards a target, the mass and depth distribution of the atoms in the target can be deduced from the energy distribution of the backscattered ions (RBS, see chapter 4.1.2). For single-crystalline materials, the incoming beam direction can be aligned with one of the major crystallographic axes of the substrate. At such conditions, called RBS channeling, the ion beam is steered into the open spaces (channels) between close packed rows or planes of atoms in the crystal (see figure 4-13).
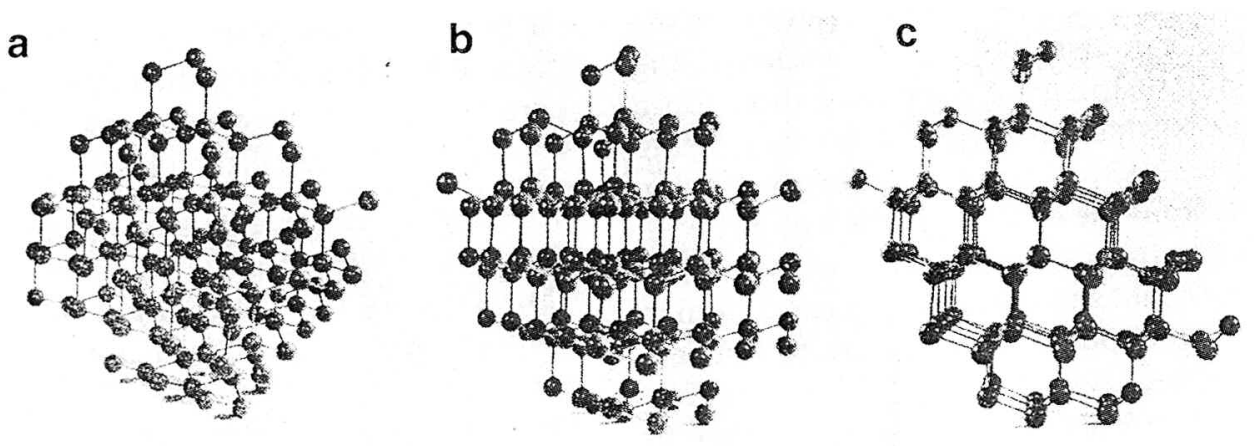

Figure 4-13: Model of lattice atoms, showing the atomic configuration in the diamond cubic structure, viewed along (a) random, (b) $\{110\}$ planar, and (c) <110>axial direction (image from [99]). 
The steering is a result of a correlated series of small-angle screened Coulomb collisions between an ion and the atoms bordering the channel. Thus, channeled ions do not come closer to the vibrating target atom nuclei than their screening distance, and the probability of large angle Rutherford collisions (backscattering) is greatly reduced compared with the probability of such interactions from a non-channeled (random) beam of ions. [100] Consequently, for ions incident at small angles $\psi$ to a close packed direction, a large reduction in the yield from such interactions with host atoms is observed. The normalized yield $\chi_{\mathrm{h}}(\psi)$ from such interactions is defined by the ratio of the yield for ions incident at an angle $\psi$ to the axial channel direction, to the yield for a randomly directed ion beam. Figure 4-14 (a) plots the normalized backscattered yield as a function of the angle $\psi$ between the ion beam and a axial channel direction. Figure 4-14 (b) shows a RBS spectrum obtained from an <100> silicon crystal in random conditions and in channeling mode. Thermal vibrations basically cause $\chi_{\mathrm{h}}$. The normalized yield $\chi_{\mathrm{h}}$ is only $4 \%$ in the surface near region. A low percentile of the ions are backscattered from the exposed surface atoms of the sample, which leads to the appearance of a small surface peak in the channeling spectrum (see figure 4-14 (b)).

a)

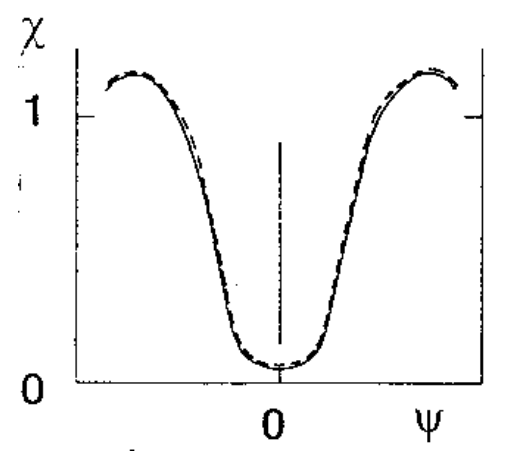

b)

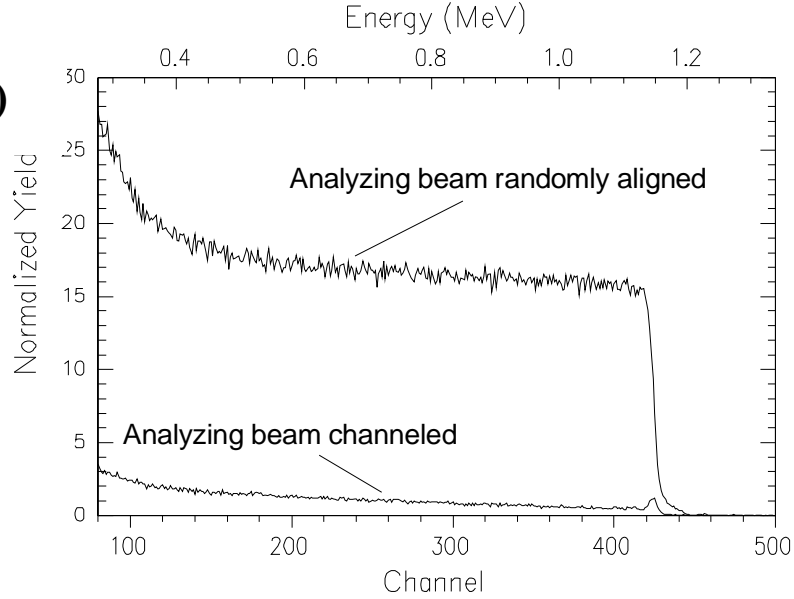

Figure 4-14: (a) Illustration of an angular scan path for an axial angular scan; (b) $2.0 \mathrm{MeV}^{4} \mathrm{He}^{+} \mathrm{RBS}$ spectrum of a untreated $\langle 100\rangle$ silicon wafer in random conditions and in channeling mode channeled along the $\langle 100\rangle$ axes. The measurements were carried out at room temperature.

The maximum angle $\psi$ between the analyzing beam and the channel direction to get ion beam steering into the channels is largely dependent on the ion energy, but also on the lattice potential and consequently on the atomic number and the distance between the target atoms. An estimate is provided by the so-called Lindhard's critical angle $\psi_{c}$ for axial channeling 


$$
\psi_{c}=\sqrt{\frac{2 Z_{1} Z_{2} e^{2}}{E \cdot d}}
$$

where $Z_{l} e$ and $E$ are the nuclear charge and kinetic energy of the incident ions, respectively. $Z_{2} e$ is the nuclear charge of the target atoms and $d$ is the distance between the atoms along the channeled axis. In the experiments for this work, a $2.0 \mathrm{MeV}{ }^{4} \mathrm{He}^{+}$ beam was used, which results in a critical angle of about $0.2^{\circ}$.

For the alignment of the silicon sample parallel to the $\langle 100\rangle$ channel direction, the <100> silicon samples are mounted on a goniometer in the accelerator target chamber. The goniometer is tilted to a small angle of $2^{\circ}$, so that the analyzing beam is incident on the target at approximately $2^{\circ}$ to the $\langle 100\rangle$ axial channel direction. Using a single channel analyzer, an energy window is set just below the maximum energy of the He ions, backscattered from the silicon crystal. The RBS counts are then collected for a given ion dose as the angle between the beam direction and the sample surface change in $0.2^{\circ}$ steps either in the $\mathrm{x}$ or the $\mathrm{y}$ direction parallel to the sample surface. The RBS counts are recorded as a function of sample tilt. The tilt positions at which mayor dips in the yield occur correspond the alignment with planar channels. Adjusting the tilt in $\mathrm{x}$ direction and in y-direction, so that they are aligned parallel to the corresponding planes, the He beam is aligned parallel to the $\langle 100>$ axial channels.

Damage to a crystal involves displaced lattice atoms, and broadly includes strain, point defects, dislocations, stacking faults, twins, defect clusters, small precipitates, and amorphous regions. If the single crystal was treated by ion-implantation the produced damage consists of Frenkel pairs (a vacant lattice site and the self-interstitial atoms ejected from that lattice site), and clusters of vacancies and self-interstitials, some of which may condense to form voids or dislocation loops. High implantation doses can even produce amorphous regions.

Displaced atoms can affect channeling in two qualitative ways (see figure 4-15). One is the dechanneling, a gradual defection of the channeled ions out of the channel. The dechanneled fraction is backscattered from all atoms in the same way as a randomly directed beam. The other influence of lattice defects on the channeled ions is direct backscattering of channeled ions by self-interstitials, which are located in the channel. Hence the channeling spectrum consists of two fractions, the dechanneled and the direct backscattered portion. [101] Figure 4-15 illustrates the different types of ion scattering from lattice defects. 

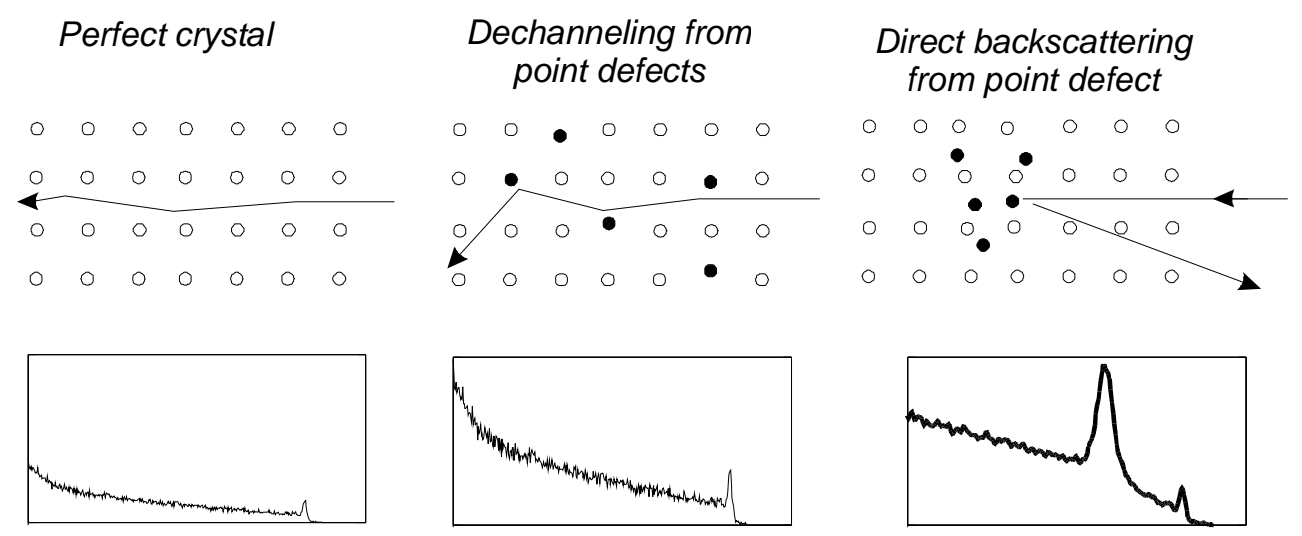

Figure 4-15: Schematic illustration of the ion scattering from defects: steering as a result of a correlated series of small-angle screened Coulomb collisions in a perfect crystal; dechanneling by multiple scattering from point defects; direct backscattering from "obstruction"-type point defects.

For alignment of the analyzing beam along a channel $\langle u v w\rangle$, the normalized yield from host atoms $\chi_{h}^{<u v w>}$ can be written as

$$
\chi_{h}^{<u v w>}=\chi_{h D}^{<u v w>}+\left(1-\chi_{h D}^{<u v w>}\right) \cdot\left(C_{h}^{<u v w} F^{<u v w>}\right)
$$

where $\chi_{h D}^{<u v w>}$ is the dechanneled fraction of the ions and $\left(1-\chi_{h D}^{<u v w>}\right)$ the channeled fractions of the ions. $C_{h}$ is the fraction of displaced atoms and $F^{<u v w>}$ the ion flux normalized with respect only to the channeled portion of the ion beam.

For the analysis of the ion-implanted species, it was necessary to quantify the effects, dechanneling and direct backscattering had on the backscattered signal. Ionimplantation induced buried damage layers can be examined by RBS channeling using an analysis based on above equation, if the crystal has not been amorphized. It is necessary to determine the dechanneled component $\chi_{h D}^{<u v w>}$ as a function of energy. The baseline of a RBS channeling spectrum indicates the dechanneled potion of the channeling yield. If the damage is located near the surface and there is a well defined peak in the aligned spectrum, then

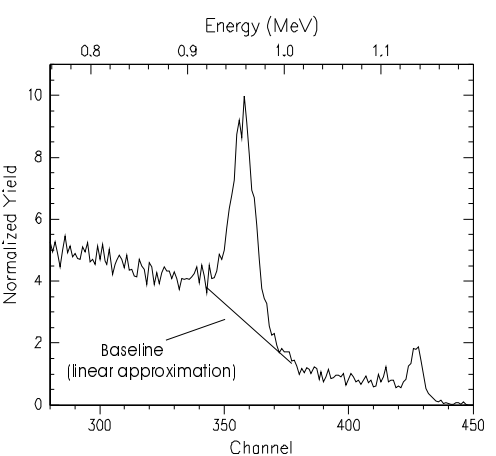

Figure 4-16: Linear approximation of the baseline for a buried damage layer. the baseline can be approximated by drawing a straight line from a point of the aligned curve near the beginning of the damaged region, to a point on the spectrum for the aligned damaged crystal just below the damaged region (linear approximation) (see figure 4-16). 
In the channeling analysis for the Ion-Cut samples, a higher accuracy for the damage profile was required. Therefore, I applied a recently developed method for the calculation of the baseline [102]. The calculation involves using an iterative procedure starting at the surface and moving in succeeding increments of depths (equal to the corresponding energy width of one channel) to determine the dechanneled fraction in the next layer, which then enables the direct backscattering contribution from displaced atoms and thus the defect density to be determined at that layer.

The examination of the mechanisms behind the Ion-Cut required accurate information not only about the damage profile, but also about the depth of the implantation damage. Therefore, the influence of the beam alignment parallel to the channel direction on the stopping power had to be considered. For this work, all channeling spectra were obtained using a $2.0 \mathrm{MeV}{ }^{4} \mathrm{He}^{2+}$ beam with a beam alignment parallel to the $\langle 100\rangle$ direction of the $\langle 100\rangle$ silicon wafers.

The depth conversion of the energy axis was performed, using recent data for the stopping power of the ${ }^{4} \mathrm{He}^{2+}$ ions in Si for random beam alignment [81] (see also chapter 4.1.1). The incoming ${ }^{4} \mathrm{He}^{2+}$ ions in the channeling direction undergo less close collisions with the target atoms and hence the stopping power for the incoming ion beam is lower compared to random beam alignment. Therefore, the stopping power $S$ was taken as $S_{\text {channeling }}=\alpha \times S_{\text {random }}$ for the incoming beam and $S_{\text {random }}$ for the outgoing beam. The factor $\alpha(\alpha<1)$ is a function of the normalized backscattering yield $\chi_{h D}$ of the dechanneled fraction of the channeling yield (i.e. the calculated baseline), which in turn was obtained from the channeling spectrum as a function of the energy of the backscattered ${ }^{4} \mathrm{He}^{2+}$ ions:

$$
\alpha=\alpha\left(\chi_{h D}(E)\right)
$$

The dependence of $\alpha$ on $\chi_{h D}$ was deduced from literature: Jin and Gibson [103] examined the amount of the channeling yield as a function of the misalignment for a 2.0 $\mathrm{MeV} \alpha$-particle beam channeled axial in the $\langle 100\rangle$ direction of a silicon crystal (the same beam-target arrangement present in this work). Their results are shown in figure 4-17a. 
(a)

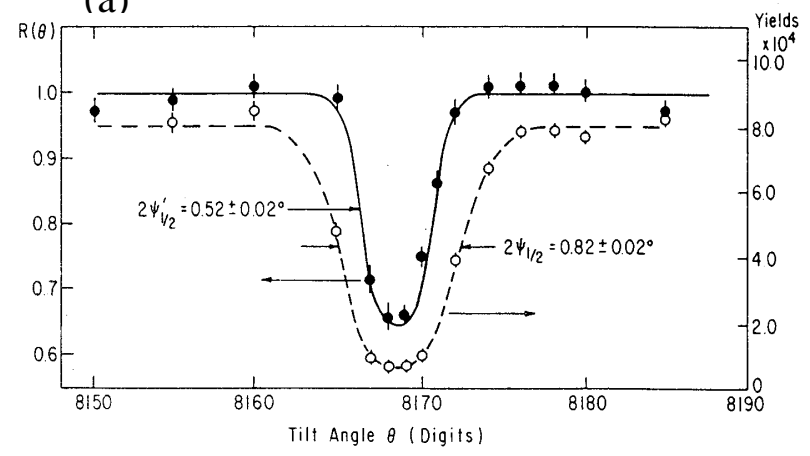

(b)

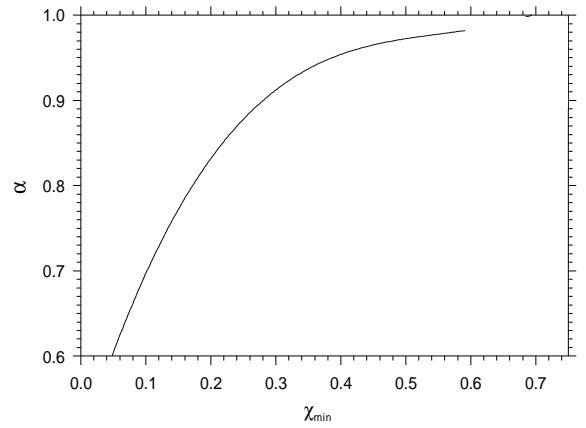

Figure 4-17: (a) The ratio $R(\theta)$ between the energy loss of $2 \mathrm{MeV}$ channeled $\mathrm{He}^{+}$ions and the random value as a function of tilt angle $\theta$ as well as the measured $\mathrm{Si}\langle 100\rangle$ axial channeling dip. (Image from [103]). (b) The factor $\alpha$ as a function of the channeling yield, deduced from (a).

The value of $\alpha$ for perfect beam alignment with the $<100>$ channel was compared to other research on stopping power of $\alpha$-particles channeled axial in the $\langle 100\rangle$ direction in silicon [104], and good agreement in the factor $\alpha$ between this work and figure 4-17b was observed.

The RBS channeling analysis was performed using the computer program Mathcad TM. Figure 4-18 shows as an example the analysis of a channeling spectrum obtained from proton implanted silicon.
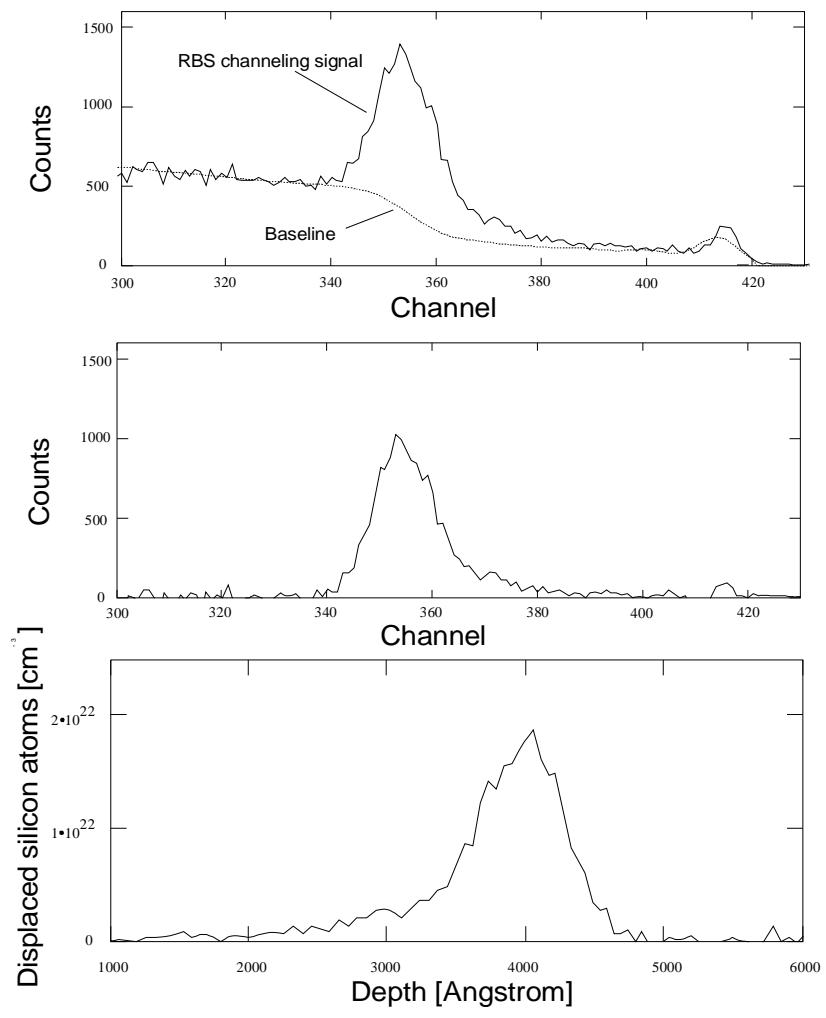

Figure 4-18: RBS channeling analysis of a silicon implanted with $40 \mathrm{keV}$ protons to a dose of $2 \times 10^{16} \mathrm{~cm}^{-2}$ and subsequently annealed at $230{ }^{\circ} \mathrm{C}$ for 30 minutes. First the baseline is calculated (a), then the baseline is subtracted from the total channeling yield (b). Finally, the energy axis is converted into a depth scale and the direct backscattering yield is normalized to the RBS spectrum taken in random condition and multiplied by the atom density of the silicon crystal. 


\subsubsection{Channeling Scanning Transmission Ion Microscopy (CSTIM)}

The technique of channeling scanning transmission ion microscopy (CSTIM) can be used to produce images of crystal defects using a focused and scanned ion beam from a nuclear microprobe $[105,106]$.

Images of micrometer-sized defects can be generated by detection of $\mathrm{MeV}$ ions from a highly focused ion beam transmitted through a thinned crystal. The CSTIM technique exploits the reduced energy loss rate which channeled ion undergo as they penetrate the crystal. A defect in the crystal locally disrupts the regular atomic arrangement and thus will affect the channeling process, producing dechanneling of initially channeled ions (see also chapter 4.2.1). The stopping power of the dechanneled fraction of the ion beam transmitting through the sample is larger than that of the fraction of the ion beam, which transmits the sample in channeling direction. Dividing the transmitted proton energy spectrum into energy intervals for each beam position on the sample produced images of the samples. Images were generated for each energy interval by showing the number of protons detected in the given energy interval at each pixel. The images obtained from all energy intervals were combined together to form the final CSTIM image showing the mean transmitted proton energy loss for each pixel. Figure 4-19 shows schematically the setup of a CSTIM.

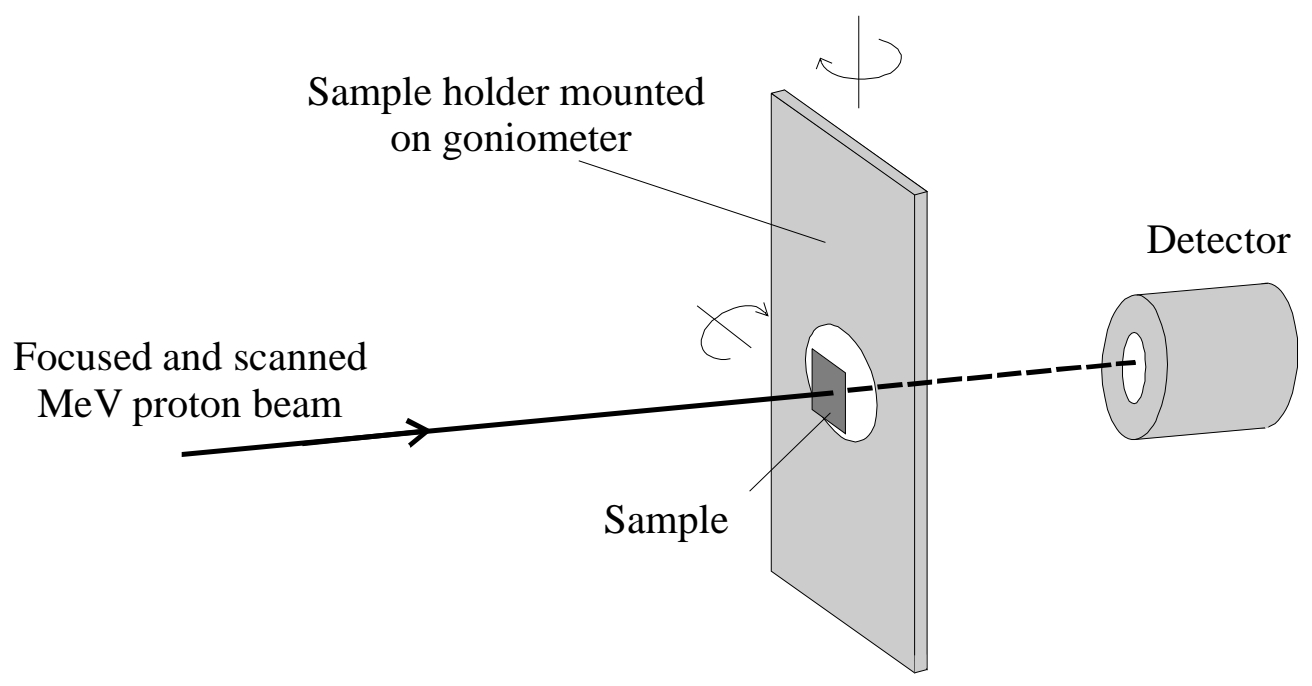

Figure 4-19: Schematic illustration of the CSTIM setup.

The incident analyzing beam particles are focused by a double focusing analyzer magnet before interacting with the sample [105]. 
CSTIM was used in this work to locally examine the damage in proton implanted and subsequently annealed samples. After the ion implantation and heat treatment, the silicon samples were prepared for CSTIM analysis by thinning from their unpolished back side using wet and dry paper, followed by diamond lapping compound to remove large scratches. The final thickness of the samples was about $20 \mu \mathrm{m}$. Afterwards, the CSTIM samples were mounted in the target chamber on a goniometer allowing rotation through a limited range of angles about two perpendicular axes in the sample plane. A semiconductor charged-particle detector is placed behind the sample in the beam axis to measure the energy of the transmitted protons. As in RBS channeling an angular scan of the sample orientation in respect to the analyzing beam was performed to align the analyzing beam parallel to the vertical [110] plane.

Figure 4-20 shows as an example the CSTIM image of a proton implanted and subsequently annealed $\langle 100\rangle$ silicon wafer. The image shows only counts of a certain energy interval. Dark areas correspond to a low number of counts, lighter areas to a higher number of counts. The three dark areas are regions of higher transmitted proton energy than the surrounding regions and are interpreted as regions, where the "popping off' of the lid of subsurface $\mathrm{H}_{2}$-gas bubbles occurred, which led to a smaller sample thickness and consequently to higher transmitted proton energies.

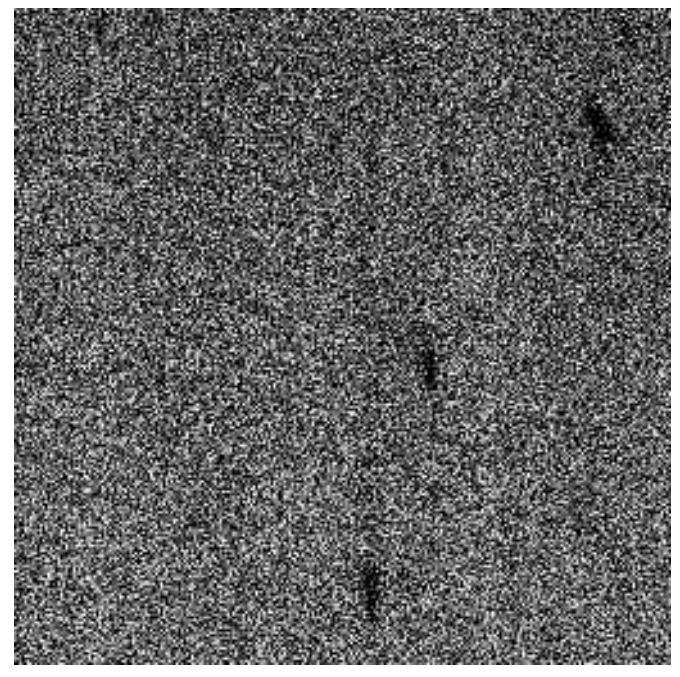

Figure 4-20: $200 \times 200 \mu \mathrm{m}$ CSTIM image of a silicon sample, planar channeled in the [110] plane. The sample was implanted with $40 \mathrm{keV}$ protons to a dose of $2 \times 10^{16} \mathrm{~cm}^{-2}$ and subsequently annealed at $410^{\circ}$ for 30 minutes. 


\subsection{Surface Topography}

\subsubsection{Scanning Electron Microscopy}

The scanning electron microscope (SEM) scans a focused electron beam across the sample surface. Scintillator-photomultiplier detectors collect backscattered electrons and/or electrons emitted from the sample. The output from the detectors is scanned synchronously with the scan of the electron beam on the sample, producing twodimensional images of the sample surface. The magnification of the SEM image equals the ratio between the distance on the sample surface scanned by the electron beam and the portrayed distance on the SEM image. Figure 4-21 shows schematic the setup of a SEM. In the SEM used for this work (JEOL model 6300 FXV) the electron beam is produced by a field emission gun. An electromagnetic condenser lens system reduces the electron beam to a highly focused electron probe. The maximum resolution is determined by the focus of the electron beam, which is specified by the manufacturer to be $1.5 \mathrm{~nm}$. The upper and lower deflection coil deflects the electron beam in a manner that the beam scans the whole specimen surface to be imaged. An aperture is placed in the electron optical column to decrease the divergence angle of the electron beam. The base pressure in the sample chamber is $10^{-6} \mathrm{mbar}$.

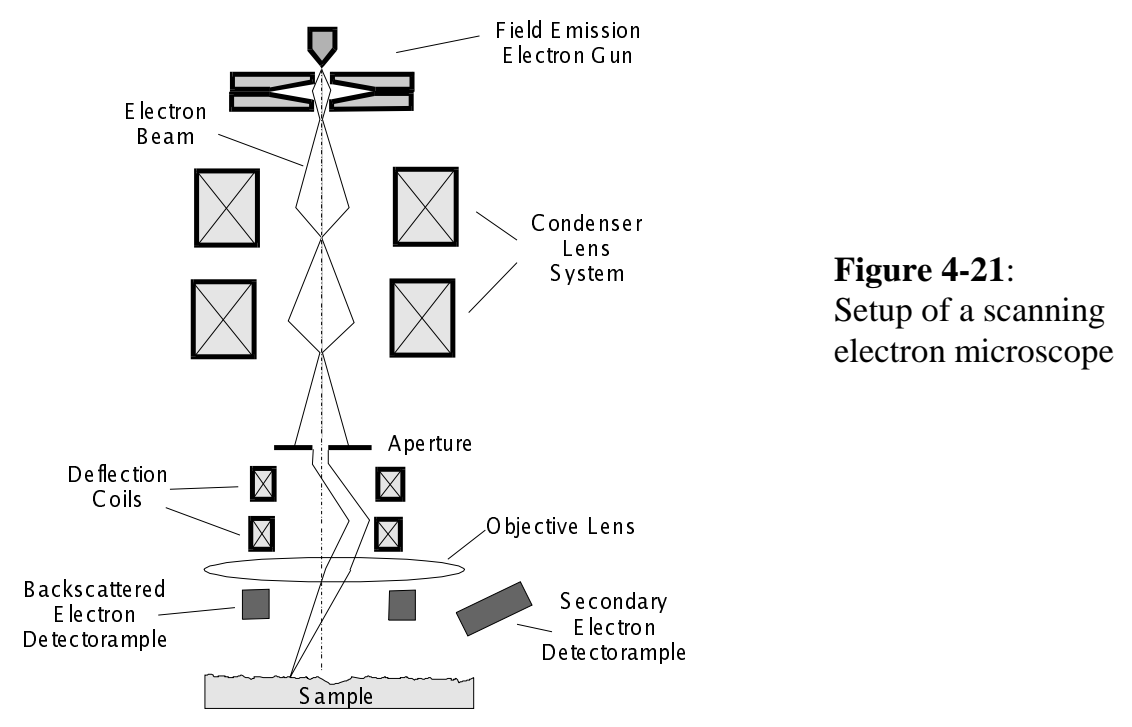

The secondary electrons with energies $\leq 50 \mathrm{eV}$ are collected to provide topographic information, whereas backscattered electrons have far higher energies and are used to gather atomic number or orientation information.

The SEM measurements in this work were carried out to gain topographic 
information. Plane view SEM was employed for the investigation of $\mathrm{H}$-implantation induced surface blistering. Cross-section SEM (XSEM) was applied to measure the thickness of exfoliated layers after ion-cutting, the depths of $\mathrm{H}$-implantation induced surface blisters and to investigate proton implantation induced silicon lattice damage by the differences in secondary electron emission characteristics of crystal phases.

For topographic measurements, only secondary electrons are collected. To ensure efficient accumulation of secondary electrons a positive biased grid is placed in front of the secondary electron detector, a scintillator-photomultiplier. The contrast arises from changes in surface topography: when the surface is locally nearly parallel with the electron beam a large number of incident electrons will be elastically scattered, generating a high secondary electron signal. Because the parts of the sample surface, which have the most direct path to the detector, appear brightest, the image of a rough specimen appears as if it is viewed from above, when being illuminated form the detector. [107] Figure 4-22 shows how XSEM depth measurements of a surface blister were obtained.
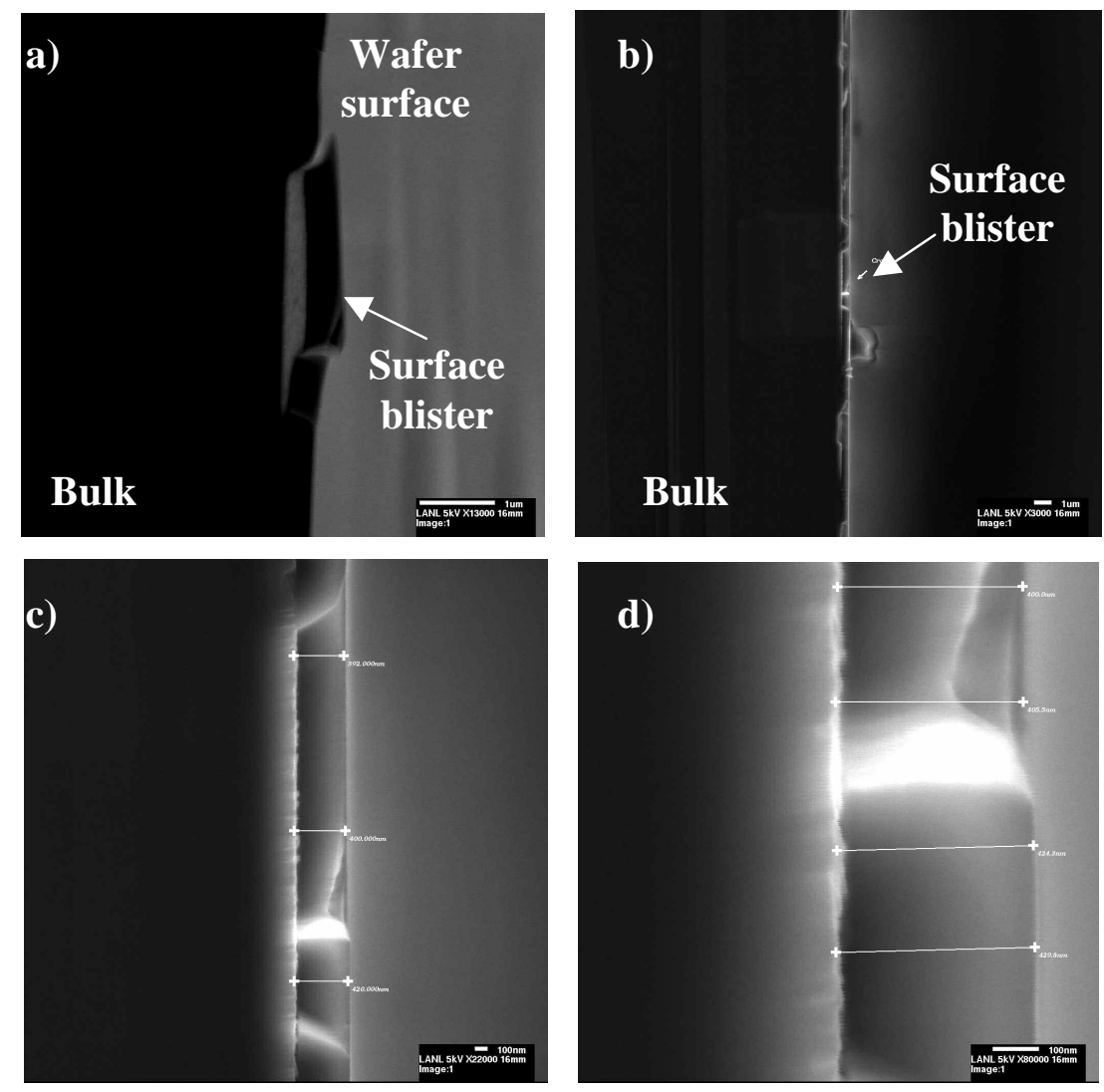

Figure 4-22: XSEM imaging of a surface blister. The silicon wafer is cut in half. The sample is oriented $45^{\circ}$ to the electron beam. The edge of the wafer is scanned until a surface blister is found, which is located exactly on the split line (a). The magnification is decreased and the sample tilted until it is oriented exactly $90^{\circ}$ to the electron beam (b). For the depth measurements the magnification is increased up to X 80000 $(\mathrm{c}, \mathrm{d})$. 
The strength of the SEM in the topographical mode lies in the very large depth of field, i. e. the distance $D$ the sample surface can be displaced from the point of focus without the sample surface going out of electron optical focus (see figure 4-23). The convergence angle $\alpha$ of the electron probe determines the depth of field of an SEM. Thus, the large depth of field $D$, which is commonly associated with SEM images, is due to the convergence angle $\alpha$ at the specimen, which is much smaller that the corresponding angles in optical microscopes.

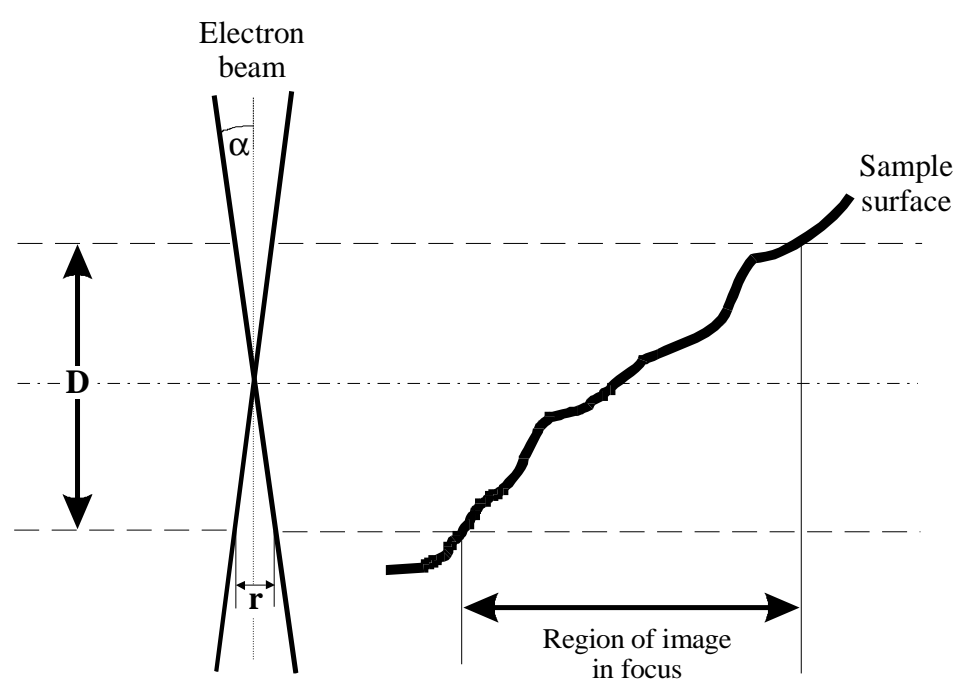

Figure 4-23: Schematic illustration of the depth of field $D$.

To a first approximation, the vertical distance $D / 2$ required to broaden the electron beam of a minimum radius $(=1.5 \mathrm{~nm}$ as specified by the manufacturer $)$ to a radius $r$ is [108]:

$$
D / 2 \cong r / \alpha
$$

The electron beam divergence $\alpha$ depends on the aperture radius $R$ and the working distance $W D$, i.e. the distance between the bottom pole piece of the objective lens and the sample surface $(\alpha=R / W D)$. For the SEM imaging in this work, an aperture with a radius of $25 \mu \mathrm{m}$ was chosen and the working distance was kept at $18 \mathrm{~mm}$ for all measurements. For a SEM image resolution of $0.1 \mathrm{~mm}$ at a magnification of 80000 , the highest magnification used in this work, the value for a sufficient small radius $r$ of the broadened electron beam is $3.75 \mathrm{~nm}$. Hence, the depth of field is calculated to be: 


$$
D=2 r / \alpha=2 r /(R / W D)=5.4 \mu \mathrm{m}
$$

This very large depth of field for such a high resolution image provides a high accuracy in the surface blister depth measurements (see figure 4-22). Viewed in cross-section, the vertical distance between the blister bottom and the back wall of the surface blister do not exceed the depth of field and consequently are both in focus. To verify the accuracy of the SEM, the microscope was calibrated before each measurement with NIST certified SEM magnification standard [109]. The error in the depth measurement was found to be less than $+/-1 \%$.

\subsubsection{Atomic Force Microscopy}

The atomic force microscope (AFM) offers the possibility to obtain the surface topography quantitatively under atmospheric conditions. In this work, atomic force microscopy was applied to measure the thickness of exfoliated layers after ion-cutting and the depths of H-implantation induced surface blisters. Furthermore, threedimensional surface topography of the silicon samples was obtained to gain quantitative information of the roughness of surfaces created by the Ion-Cut. The roughness values can be gained from the individual height positions $\mathrm{Z}(\mathrm{X}, \mathrm{Y})$ of the sample surface by various averaging methods. In this work the AFM results were quantified by the root mean square (rms) value of the surface roughness:

$$
R_{r m s}=\sqrt{\frac{\sum_{n=1}^{N}\left(z_{n}-\bar{z}\right)^{2}}{N-1}}
$$

where $\mathrm{N}$ is the number of data points and $\bar{z}$ is the mean height.

The functionality of the AFM is based on the interaction of a very thin tip (radius $<50 \mathrm{~nm}$ ) with the potential of the sample surface. The setup of an AFM is presented in figure 4-24. The tip is mounted on a cantilever spring and scans the sample surface. The electron cloud of the tip pushes against the clouds of individual atoms in the sample, generating a repulsive force that varies with the surface topography. The force deflects the tip. These tip movements are monitored by a laser beam deflected from the top of the cantilever to a position sensitive photo detector. The AFM was used in the socalled "contact mode", where the tip and sample remain in close contact during the scan. 


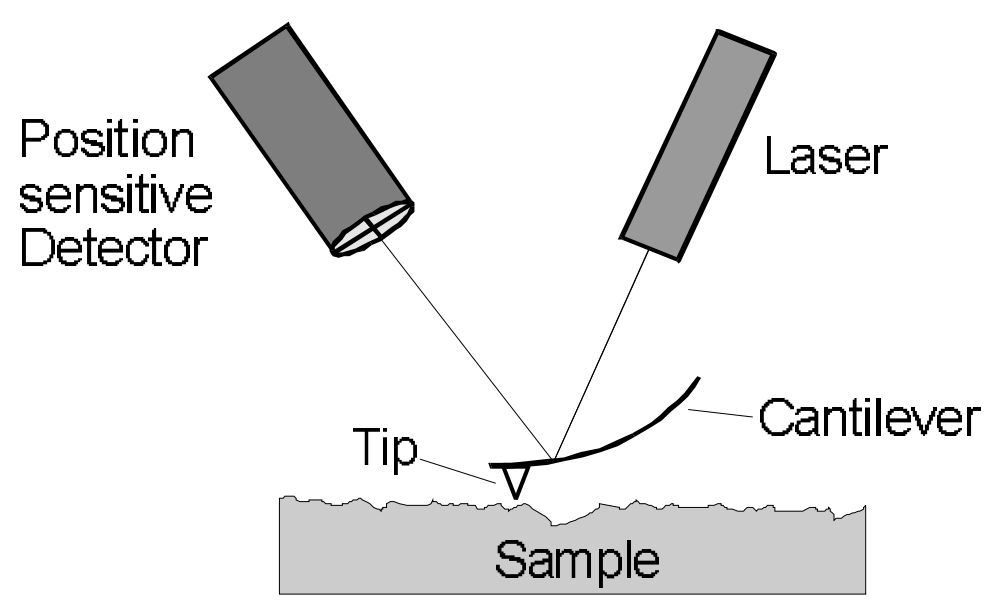

Figure 4-24: Schematic illustration of the AFM measurement setup

The AFM can be operated in two principal operation modes, with or without feedback control. Here, the AFM operated with feedback control, the so-called "constant-force" mode. The piezoelectric control, which is moving the sample or tip (here the tip) up and down, responds to any changes in the position where the laser beam hits the position sensitive photo detector. It adjusts the tip's height, so that the position of the laser beam on the detector remains constant, keeping the bending of the cantilever and consequently the force between the tip and the sample to a pre-determined value. The tip's movements are converted into a three-dimensional surface profile.

A Park Scientific Instruments AUTOPROBE M5 ${ }^{\mathrm{TM}}$ AFM was used. The tips were made of silicon nitride and had a diameter of only 20 to 50 Angstrom, which allowed for a high resolution of the AFM images. The line scan frequency was set to $0.5 \mathrm{~Hz}$. As an example figure 4-25 shows a typical AFM image, obtained from an IonCut generated silicon surface.

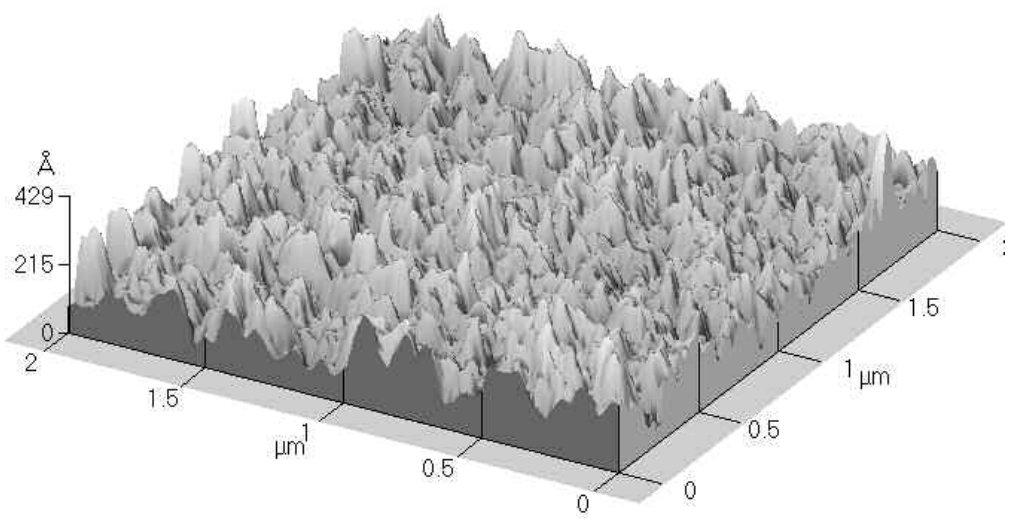

Figure 4-25: AFM image of the silicon surface created by the Ion-Cut. The sample was implanted with 50 $\mathrm{keV}$ protons, subsequently bonded to a stiffener and finally annealed for 75 seconds at $600{ }^{\circ} \mathrm{C}$. 


\subsection{Microstructure: Transmission Electron Microscopy}

Transmission electron microscopy (TEM) is used to obtain information from samples, which are thin enough to transmit electrons. Because of its excellent resolution $(\approx 0.2$ $\mathrm{nm}$ ), the TEM provides access to the microstructure down to molecular dimensions. Figure 4-26 shows a schematic setup of a TEM. A beam of highly focused electrons is directed towards a thinned sample. The transmitted electrons penetrate an objective lens and subsequent imaging lenses before they hit the photo plate. An objective aperture is inserted in the back focal plane of the objective lens. This aperture is used to select the electron beam area from which to form the image. A variety of different signals can be used for image formation, among these transmitted and diffracted electrons as well as characteristic X-rays, allowing for a unique information combination concerning shape, distribution, crystallography and chemistry of microstructural detail.

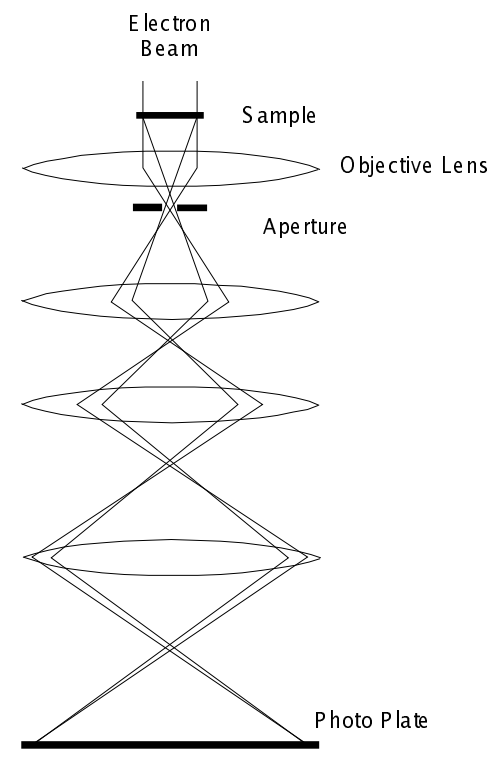

Figure 4-26:

Setup of a transmission electron microscope. (Here, the aperture is positioned to provide a brightfield image.)

Depending on the positioning of the aperture slits the TEM imagining is divided in two categories. In the bright-field image the aperture blocks the diffracted electron beam and only the incident beam is let through on the photo plate. On the contrary, when the incident beam is deflected so that only the diffracted electrons hit the photo plate, an electron diffraction pattern is obtained. In both cases a purely amplitude contrast image is obtained. A major advantage of microstructure analysis by TEM compared to microstructure analysis by X-ray diffraction (XRD) is the ability to analyze very small 
crystal areas down to a diameter of $50-200 \mathrm{~nm}$. [107] The strong interaction of electrons with the nuclei increases the intensity of the reflections.

In this work TEM was carried out only in the cross-section mode (XTEM). Bright field images were obtained to determine the depth of the implantation zone and to gain detailed information about the crystal defects within the implantation zone (dislocations, $\mathrm{H}$-decorated platelets, etc.). Electron diffraction patterns were obtained to gain knowledge about the crystalline quality of the silicon within the implantation zone and also in the surface near region above the implantation zone. The TEM analyses were carried out employing the TEM, type Philips CM30 T, operated with an acceleration voltage of $300 \mathrm{kV}$.

The penetration depth of the electrons in the sample is limited and therefore only very thin samples can be analyzed. Hence a requirement for TEM analysis is the thinning of the sample to a thickness of only about 50-100 nm. Figure 4-27 shows schematically the 4 steps of the sample preparation for XTEM analysis.

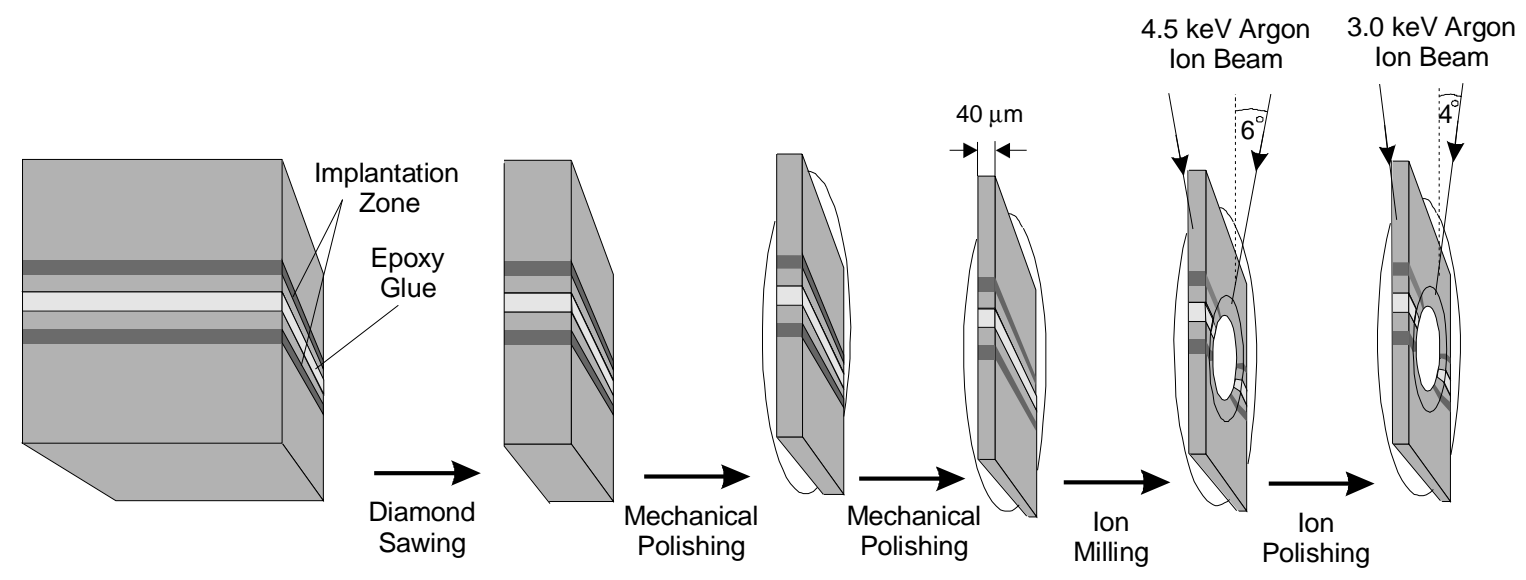

Figure 4-27: Sample preparation process for XTEM imaging.

First, the silicon sample is cut in half and the two pieces are glued together with an epoxy glue. Then, a little piece is cut out with a diamond saw and subsequently mechanically polished on one side. Then, the TEM sample is glued to a washer with the polished side facing the washer and is thinned mechanically to a thickness of about $40 \mu \mathrm{m}$ by polishing the other side of the TEM sample. Afterwards, the sample is placed in an ion-miller on a rotation stage and bombarded with a $4.5 \mathrm{keV}$ Argon beam on both side. The angle between the upper and lower sample surface and the two ion beams are $6^{\circ}$. The argon bombardment creates a crater in the TEM sample, whose edge runs across the cross-section of the sample. In the vicinity of this crater the sample is thin enough for high energetic electrons to penetrate and consequently makes XTEM analysis possible. 
Finally, the TEM sample is further polished with a $3 \mathrm{keV}$ Argon ion beam impinging on the TEM sample surface at a glancing angle of $4^{\circ}$. As an example figure 4-28 presents a bright-field XTEM image of a proton implanted silicon wafer.

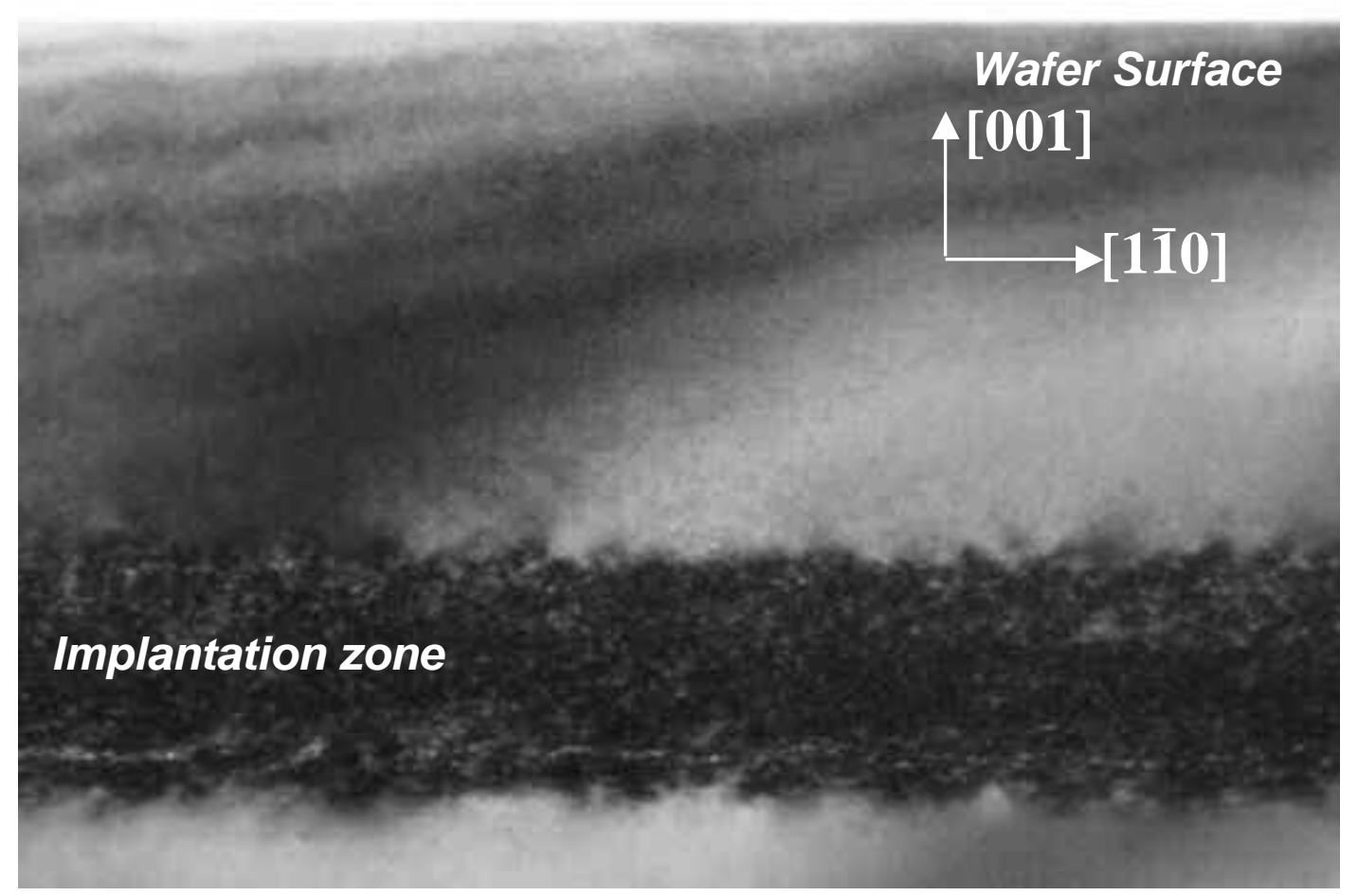

Figure 4-28: Low magnification bright field image of a $<100>$ silicon wafer, implanted at 77 Kelvin with $40 \mathrm{keV}$ proton ions to a dose of $1 \times 10^{17} \mathrm{~cm}^{-2}$. The sample was viewed edge-on in the [110] Si projection 


\subsection{Stress Measurements}

During the proton implantation compressive biaxial stresses develop due to both displacement damage and the incorporation of the implantation species. These ionimplantation induced compressive stresses in the subsurface region of the silicon wafers can be deduced by measuring the wafer curvature change due to the ion implantation process with a laser scanning setup.

Fig. 4-29 illustrates the cause of the wafer curvature for a one-dimensional case of a thin layer of thickness $t_{l}$ on a substrate of thickness $t_{s}$. The unconstrained length of the film would be $L_{l}$, but it must contract by an amount $\Delta L$ to match the length $L_{s}$ of the substrate. In the case of proton implantation, this length difference $\Delta L$ is due to the compressive biaxial stresses. The deformed layer attached to an undeformed substrate does not represent the condition of minimum energy. [110] The energy of the entire system can be lowered by deforming the substrate slightly so as to reduce the deformation of the layer. The distance $R$ from the center of curvature to the material surface quantifies this deformation.

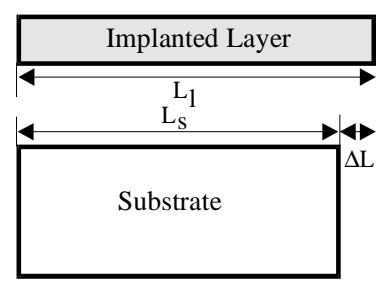

Unconstrained lengths of implanted layer and substrate

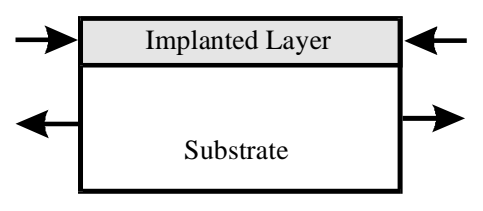

Constrained lengths of implanted layer and substrate lead to compressive stress in the implanted layer and tensile strain in the substrate

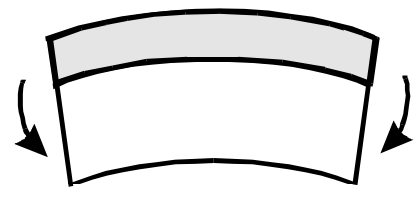

Compressive stress and tensile strain lead to bending of the sample

Figure 4-29: Silicon wafer curvature under the influence of compressive biaxial stresses in the implantation zone

The magnitude of the stress in the surface layer (here the implantation zone) can be determined using the Stoney equation [111]:

$$
\sigma=\left(\frac{E_{s}}{1-v_{s}}\right) \cdot \frac{t_{s}^{2}}{6 t_{l}} \cdot \frac{1}{R}=Y_{s} \cdot \frac{t_{s}^{2}}{6 t_{l}} \cdot \frac{1}{R}
$$

Here, $\sigma$ is the stress in the surface layer, $E_{s}$ is the biaxial modulus of the substrate, $v_{s}$ is the Poisson ratio of the substrate, $t_{s}$ is the thickness of the substrate and $t_{l}$ the thickness of the surface layer, $R$ is the radius of the curvature, and $Y_{s}$ is the biaxial stress state elastic modulus of the substrate $\left(Y=1.805 \times 10^{11} \mathrm{~N} / \mathrm{m}^{2}\right.$ for $(100)$ silicon). However, because the 
substrate, here a silicon wafer, has some finite amount of curvature prior to the proton implantation, the Stoney equation must be modified so as to account for the differences in curvature. Therefore, the stress in the implanted layer is actually calculated using the difference between the curvature parameters of implanted and untreated silicon substrate:

$$
\sigma==Y_{s} \cdot \frac{t_{s}^{2}}{6 t_{l}} \cdot\left(\frac{1}{R_{\text {implanted }}}-\frac{1}{R_{\text {untreated }}}\right)
$$

where $R_{\text {untreated }}$ and $R_{\text {implanted }}$ are the curvature radii of the silicon wafer before and after ion implantation, respectively. The silicon samples used for stress measurements were cut parallel to the $\{110\}$ planes into a rectangular shaped stress bar $(5 \mathrm{~mm} \times 38 \mathrm{~mm} \times 0.4 \mathrm{~mm})$.

Figure 4-30 shows a schematic diagram of the component layout and the beam path from the laser head to the specimen and back to the detector. The HeNe laser has an output of $2 \mathrm{~mW}$ and the wavelength of the exit radiation is $632.8 \mathrm{~nm}$. The output is attenuated through a variable attenuator. The attenuated beam strikes the scanning mirror, which is driven by a scanning motor and provides the scan angle and the scan distance on the silicon sample. The scanned beam is directed towards the first mirror, passes through a beam splitter, and is scanned across a 1 meter focal point lens. This lens is exactly one meter away from the scanning point, and exactly one meter away from the linear position-sensitive detector. The light beams exciting from the lens and towards the silicon sample are all parallel to each other and the optical axis.

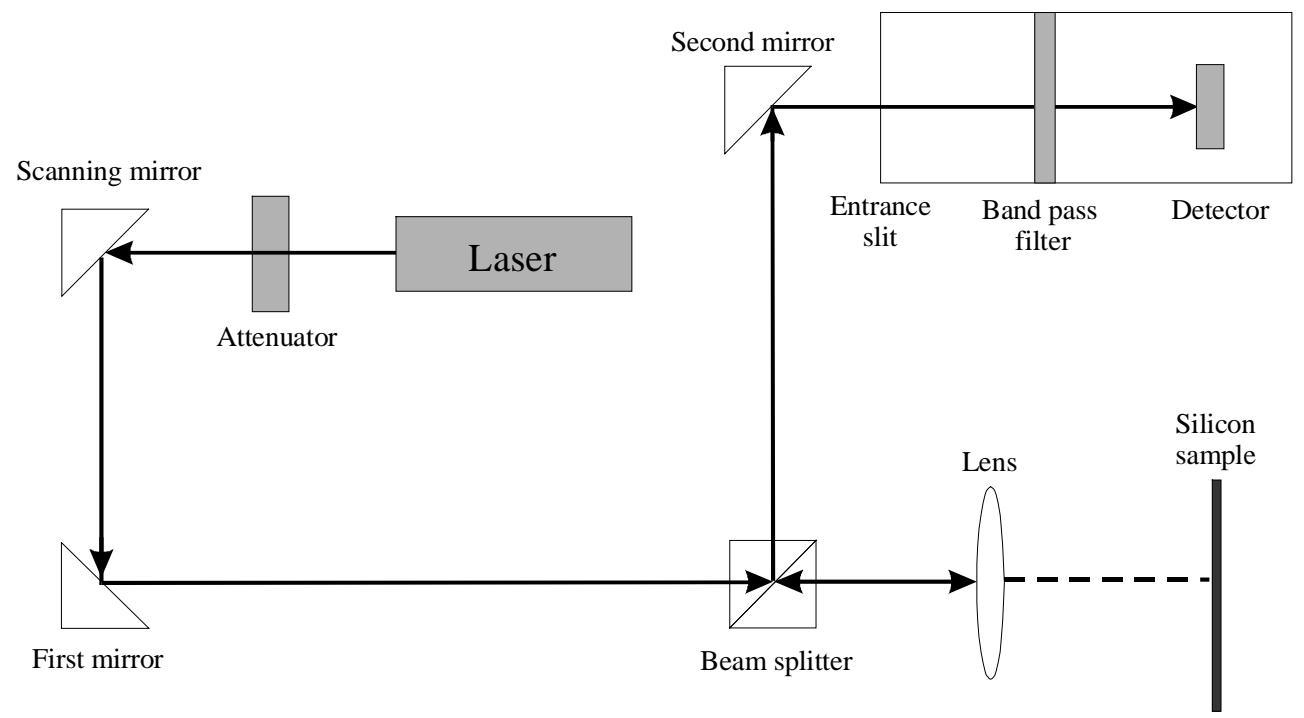

Figure 4-30: Schematic illustration of the laser scanning setup 
The discrete scan positions on the silicon sample are reflected back through the lens, towards the beam splitter and onto the second stationary mirror. The second mirror directs the reflected beam towards the detector assembly. The detector assembly consists of an entrance slit and a narrow band pass filter. The slit cuts down the amount of stray light that reaches the filter and the filter allows only light of a wavelength of between 632.8 and $633.3 \mathrm{~nm}$ to the position-sensitive detector. The position-sensitive detector is capable of resolving displacements in the laser position as small as $0.3 \mu \mathrm{m}$.

The relationship between the change in the position of the laser spot $\Delta d$, the scan length on the silicon sample $\Delta s$, the focal length $f$ and the radius of curvature $R$ is given by [112]:

$$
\Delta d=\frac{2 f}{R} \Delta s=m \Delta s
$$

where $\mathrm{m}$ represents the slope of the line given by $\Delta d$ versus $\Delta s$. 


\section{The Mechanisms behind the Ion-Cut Process}

As outlined in chapter 2.1 the process of hydrogen induced silicon surface layer exfoliation has been investigated by a variety of spectroscopic methods, to achieve knowledge about how hydrogen interacts with silicon at the atomic level. However, at a very fundamental level the basic mechanisms behind the Ion-Cut process are still unclear. The general belief has been that the silicon cleavage takes place at the peak in the $\mathrm{H}$ implantation concentration profile. The aim of the present study is to explore the influence of the hydrogen-concentration depth distribution and implantation-damage depth distributions on the Ion-Cut location in hydrogen implanted silicon. This information turns out to be crucial in understanding the development of H-platelet formation, $\mathrm{H}_{2}$-gas bubble growth and cleavage of the silicon wafer.

The standard $\mathrm{H}$-ion implantation dose in the Ion-Cut application to SOI is $5.5 \times 10^{16} \mathrm{~cm}^{-2}$. At this implantation dose cleavage of the $\mathrm{H}$-implanted silicon wafer can be accomplished easily by annealing at around $400{ }^{\circ} \mathrm{C}$ for several minutes [9], depending on the conductivity type of the silicon and the dopant concentration. However, additional information about the cleavage mechanisms can be learned by performing Ion-Cut experiments on samples exposed to lower and higher $\mathrm{H}$-ion implantation doses. Such studies have shown that a minor decrease of the H-implantation dose from the standard dose requires considerable higher anneal temperatures and anneal durations to achieve silicon cleavage. Therefore, a H-implantation dose of $5.0 \times 10^{16} \mathrm{~cm}^{-2}$ was chosen for the low dose implant. When examining the influence of the H-implantation doses on the silicon cleavage (chapter 5.2), the silicon wafers were implanted with 5, 7, 10 and $20 \times 10^{16} \mathrm{H} / \mathrm{cm}^{2}$, respectively. In chapter 5.3 the influence of implantation parameters like implantation energy and implantation temperature and the influence of the method, the Ion-Cut is induced, were investigated. 


\subsection{Low Dose Hydrogen Implantation}

The substrate used in this experiment was a $<100>$ oriented p-type silicon wafer (boron doped) with a resistivity of 5-50 $\Omega \mathrm{cm}$. This corresponds to a boron dopant level of $2.7 \times 10^{14} \mathrm{~cm}^{-3}$ to $2.5 \times 10^{15} \mathrm{~cm}^{-3}$. The sample was implanted with $175 \mathrm{keV}$ protons to a dose of $5 \times 10^{16} \mathrm{~cm}^{-2}$. The ion implantation was performed at room temperature. To minimize channeling effects, the incident ion beam was oriented at 7 degrees from the surface normal. Following the ion implantation the implanted wafer was bonded to the stiffener, a <100> oriented silicon wafer with a $75 \mathrm{~nm}$ thick top layer, a hydrogen silsesquioxane-based flowable oxide ('FOx') [113]. Prior to the hydrophilic bonding the $\mathrm{H}$-implanted silicon wafer underwent a standard RCA clean. To achieve strong chemical bonds between the two wafers the bonded pair was further annealed at a temperature of $70{ }^{\circ} \mathrm{C}$ for 2 hours $+200{ }^{\circ} \mathrm{C}$ for 3 hours. Finally, the two bonded wafers were annealed at $600{ }^{\circ} \mathrm{C}$ for 5 minutes inducing the complete delamination of the hydrogen-implanted wafer. Figure 5-1 shows schematically the four steps of the sample treatment.

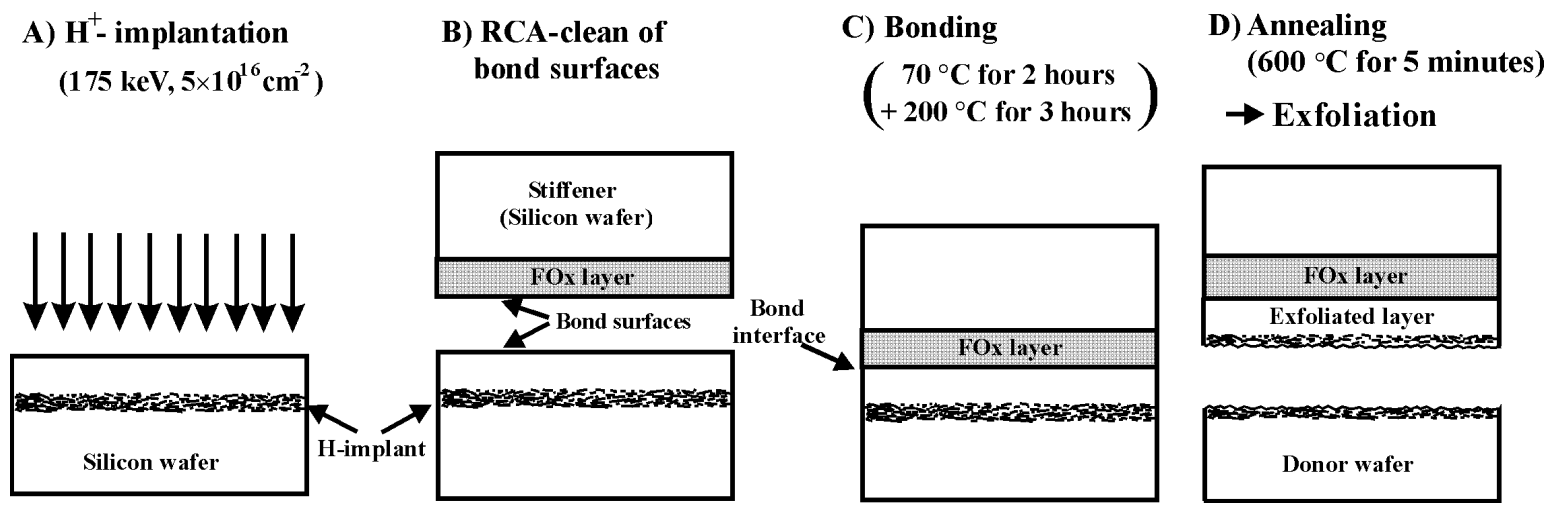

Figure 5-1: Schematic illustration of the sample preparation.

The sample was characterized in detail in the as-implanted state and also after the bonding to the handle wafer and ion-cutting was accomplished. For the sample analysis a combination of RBS channeling, ERD, XSEM, XTEM, AFM and laser scanning wafer curvature measurements was employed. 


\subsubsection{Implantation Damage Depth Profile before and after the Ion-Cut}

Rutherford backscattering spectroscopy (RBS) in the channeling mode was used to analyze the radiation-induced damage accumulation. These measurements were obtained with a $2.0 \mathrm{MeV}^{4} \mathrm{He}^{+}$analyzing beam and a detector located 13 degrees from the incident beam.

Figure 5-2 shows the ion channeling backscattering spectra obtained from the asimplanted wafer (upper part), the exfoliated layer, and the donor wafer (lower part) (see also figure 5-1). For comparison a spectrum in channeling condition and a random spectrum of a virgin silicon is also shown in the upper part of figure 5-2.

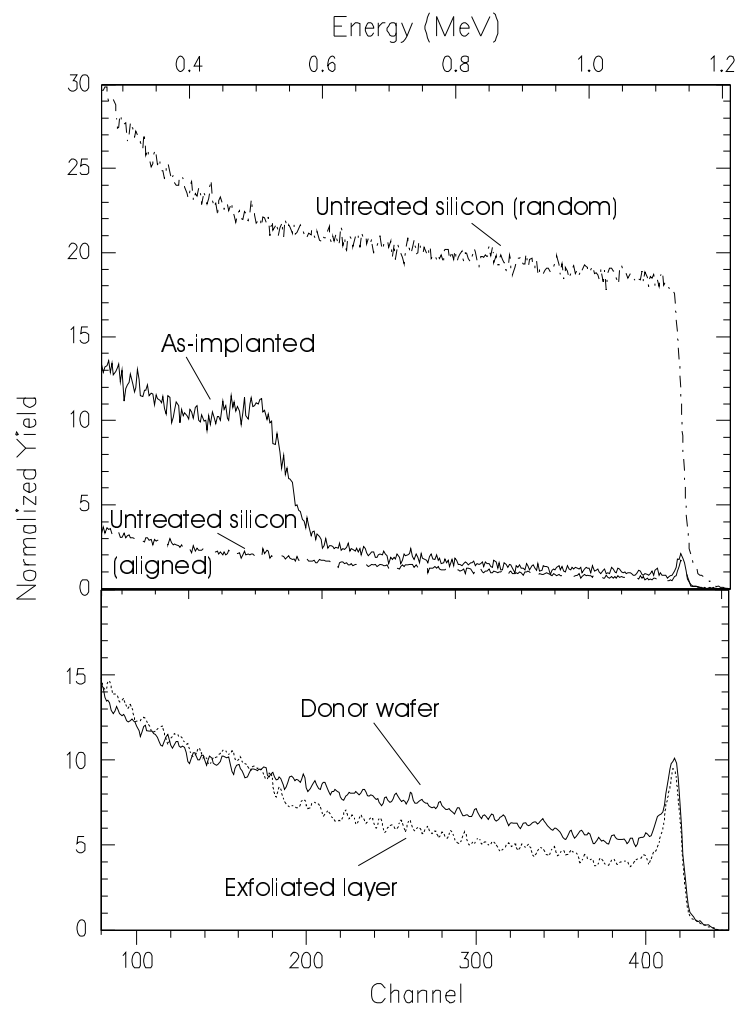

Figure 5-2: $2.0 \mathrm{MeV} \mathrm{He}$ RBSchanneling spectra of the $\mathrm{H}$-implanted $<100\rangle \mathrm{Si}$ in the as-implanted state (a) and after bonding and exfoliation (b). Also shown for comparison are a random spectrum and the channeling spectrum of the virgin silicon.

The peak in the spectrum of the as-implanted sample located between channel 140 and 210 indicates lattice damage induced by the hydrogen implantation. The backscattering yield $\chi_{\min }$ of the as-implanted sample at higher channel numbers (220-415) is quite low $\left(4.0 \%<\chi_{\min }<9.5 \%\right.$ of the random signal), revealing the high crystal quality of the silicon layer above the implantation damage region. The low amount of implantation damage in this region is due to the stopping of the $\mathrm{H}$-ions during the implantation process, which is predominated by the electronic interaction of the incoming ions with the target atoms at higher energies (see figure 2-4). This is essential for the production of 
SOI, since a high crystalline quality of the silicon layer must be provided for the production of electronic devices.

An examination of the backscattering spectra in the lower part of figure 5-2 from the exfoliated layer and the donor wafer (see also figure 5-1 (d)) shows that following the Ion-Cut process the original implantation damage in the as-implanted sample now resides at the near surface region, around channel 415. The damage peaks in the near surface area of the exfoliated layer and the donor wafer are almost equal in height. The donor wafer shows a slightly larger surface damage peak compared to the exfoliated layer. The spectrum from the exfoliated layer shows a step increase in the backscattering yield at channel 175. This step increase indicates the bond interface: Although, the silicon content in the FOx layer is low, it's amorphous structure leads to a small increase in the backscattering yield. The higher yield is maintained at lower channel numbers. This is a result of the crystallographic misalignment between the exfoliated layer and the stiffener. The channeling condition was obtained for the orientation of the $\langle 100\rangle$ channels in the exfoliated layer, not in the stiffener. Angle dependent channeling measurements showed that the misalignment between the bonded exfoliated layer and the stiffener is 0.3 degrees.

The depth distribution of the implantation damage in the as-implanted sample and the depth of the bond interface after ion-cutting (see figure 5-1 (a) and 5-1(d)) were determined by analyzing the obtained channeling data (figure 5-2) as described in detail in chapter 4.2.1.

For the calculation of the implantation damage depth recent data for the stopping power of the ${ }^{4} \mathrm{He}^{2+}$ ions in $\mathrm{Si}$ at random conditions (outgoing analyzing beam) were used. [81] The stopping power for the incoming ${ }^{4} \mathrm{He}^{2+}$ ions in the channeling direction

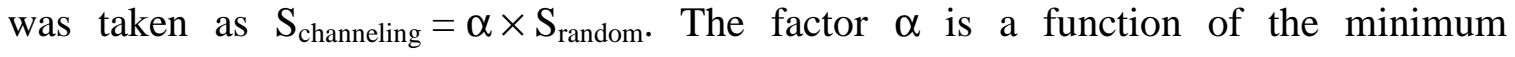
backscattering yield $\chi_{\min }$, which in turn was obtained from the channeling spectrum as a function of the energy of the backscattered ${ }^{4} \mathrm{He}^{2+}$ ions $\alpha=\alpha\left(\chi_{\min }(\mathrm{E})\right.$ ) (see also chapter 4.2.1). The value of $\alpha$ was 0.62 at the surface (channel 415) and increased up to 0.72 at channel 210.

Figure 5-3 shows the result of the analysis. The upper part includes the channeling spectra and the baseline for the as-implanted spectrum. Note that the $\mathrm{x}$-axis is already converted into a depth scale. The lower part of figure 5-3 shows the displacedatoms depth distribution. The channeling calculations reveal that the implantation damage peak is located at a depth of $1.41 \mu \mathrm{m}$. The half height position of the step in the spectrum taken at the exfoliated layer, figure 5-2, is indicated by the dotted line in the lower part of figure 5-3. This step represents the bond interface and conclusively the 
calculated depth of this step is equal to the thickness of the exfoliated layer. It is measured to be $1.39 \mu \mathrm{m}$ deep.

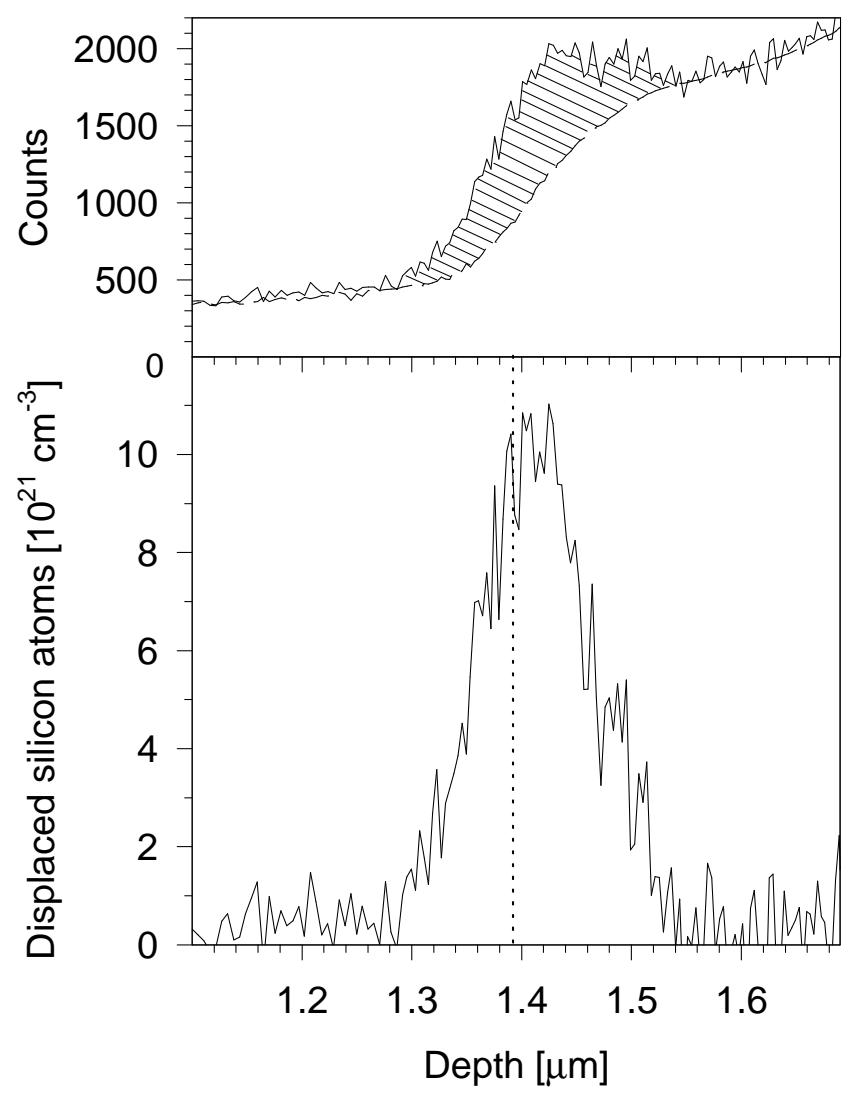

Figure 5-3: Result of the RBS channelling analysis of the asimplanted sample. The upper part shows the obtained baseline; the lower part presents the calculated damage concentration as a function of sample depth. The vertical dotted line represents the calculated thickness of the exfoliated layer.

Comparing the displaced atoms depth distribution in the as-implanted sample with the calculated depth of the bond interface after ion-cutting, the RBS channeling depth analysis reveals that the Ion-Cut takes place at a depth region slightly shallower than the implantation damage peak in the as-implanted sample.

The lower part of figure 5-2 shows the RBS channeling spectra obtained at the exfoliated layer and the donor wafer. Interestingly, the direct backscattering yield is slightly larger in the donor wafer than in the exfoliated layer. The width of the damaged region was deduced by the same calculation method discussed above and is measured to be $180 \mathrm{~nm}$ for the donor wafer and $140 \mathrm{~nm}$ for the exfoliated layer. This also indicates that the delamination of the $\mathrm{H}$-implanted silicon wafer occurred at a depth slightly shallower than the damage peak corroborating the interpretation of the data presented in figure 5-3. 
The depth distribution of the implantation damage before cleavage was only analyzed in the as-implanted sample. However, it has to be clarified whether the damage depth distribution changes due to the heat treatment during the bonding and ion-cutting. To achieve this, an as-implanted silicon wafer was exposed to the same heat treatment the bonded sample underwent during bonding and ion-cutting, but without bonding the wafer to the stiffener.

When the H-implanted silicon wafer is not bonded to a stiffener, cleavage of the whole wafer does not occur. Instead individual $\mathrm{H}$-bubbles will force the discrete "popping off" of localized regions resulting in a blistered surface appearance. [114] To avoid $\mathrm{H}_{2}$ gas bubble growth during the anneal sequence hydrogen ions were implanted into the silicon wafer with the same implantation parameters used for the bonded sample, but with a slightly reduced H-implantation dose of $4.5 \times 10^{16} \mathrm{~cm}^{-2}$. This H-implanted sample was then exposed to the same heat treatment the Ion-Cut sample underwent, but without bonding to a stiffener. The analysis of the obtained RBS channeling data was performed in the same way as described above.

The damage distribution from the non-bonded silicon, both before and after annealing are presented in figure 5-4. These data show that the damage peak shifts $10 \mathrm{~nm}$ towards larger depth during the anneal, from $1.41 \mu \mathrm{m}$ to $1.42 \mu \mathrm{m}$. The damage peak in the annealed sample is larger compared to the as-implanted sample.

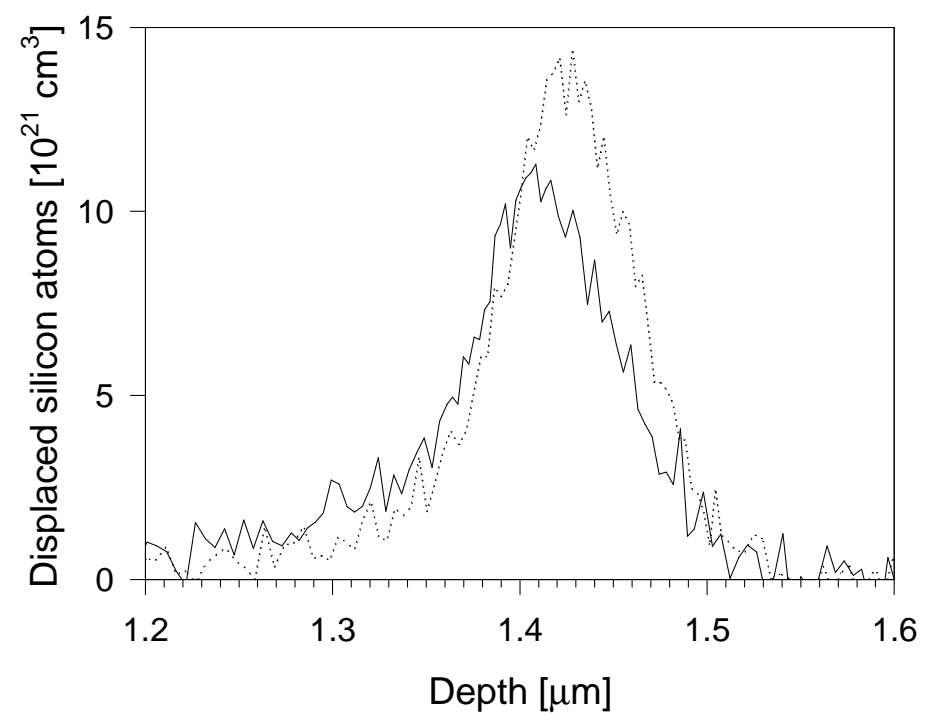

Figure 5-4: In-depth distribution of the displaced atoms in the as-implanted sample ( $\longrightarrow$ ) and after annealing for 3 hours at $200^{\circ} \mathrm{C}+5$ minutes at $600^{\circ} \mathrm{C}(---)$. The hydrogen implantation dose was $4.5 \times 10^{16} \mathrm{~cm}^{-2}$. 
The increased number of the displacements at the peak of the damage depth distribution is caused by a rearrangement of the implanted hydrogen atoms. [42] Hydrogen complexes that formed during the heat treatment have been shown to be responsible for a substantial amount of the detected damage. [42] These hydrogen complexes include $\mathrm{H}_{2}$ molecules, residing in the silicon lattice and accumulated in $\mathrm{H}_{2}$-gas bubbles.

Experimental studies have shown, that light ion implantation damage in crystalline silicon anneals out at the temperatures applied in the present heat treatment. [115] It is my belief however, that the defect-decorating hydrogen atoms prevent the full recombination of Frenkel pairs. Hence, the recombination of Frenkel pairs would mainly take place in the near surface region of the implantation damage zone where the hydrogen concentration is low. I assume that the recovery of implantation damage in the shallow part of the damage zone combined with the formation of hydrogen defect complexes causes the damage peak to shift towards a larger depth.

Considering the observed shift of the damage peak of $10 \mathrm{~nm}$ towards the bulk, the data suggest, that at the moment of the Ion-Cut, the cleavage occurs in the region of maximal damage or even slightly shallower.

\subsubsection{Depth Measurement of the Ion-Cut}

To assess the mechanisms behind the Ion-Cut, it is necessary to gain knowledge about the location within the implantation zone, where ion-cutting took place. Since theory predicts that the depth of the implantation damage peak and the hydrogen implantation peak differ only slightly $[68,116]$, is it essential to achieve accuracy in the measurement of the Ion-Cut depth. Therefore, in addition to the measurement of the Ion-Cut depth by RBS channeling (chapter 5.1.1), also electron microscopy and atomic force microscopy were applied to verify the obtained thickness of the exfoliated layer.

To determine the thickness of the exfoliated layer by SEM the stiffener with the silicon layer on top was imaged in cross-section at the edge of the sample surface. Figure 5-5 presents a high resolution SEM cross-section image of the exfoliated layer. The image shows the roughness of the surface, where the Ion-Cut occurred, to be quite small. Also visible is the $75 \mathrm{~nm}$ thick FOx layer beneath the bond interface. The large 


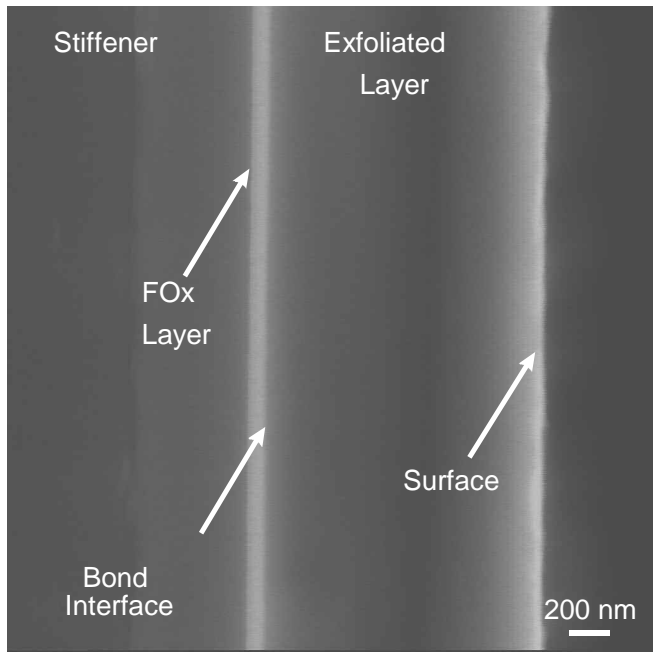

Figure 5-5: Cross-section SEM image of the exfoliated silicon surface layer after bonding and ion-cutting.

area analysis possible with SEM reveals a very uniform thickness of the exfoliated layer and consequently a very smooth cut of the H-implanted silicon crystal. The thickness of the exfoliated layer is measured to be $1.416 \mu \mathrm{m}$. Due to the low error in the depth measurements by SEM $(< \pm 1 \%)$ I assume that the present approach provides a quite exact value for the depth of the exfoliated layer. The thickness of the exfoliated layer, obtained by SEM, is in good agreement with the thickness deduced from the RBS channeling spectrum of the exfoliated layer $(1.39 \mu \mathrm{m})$.

XTEM imaging was also carried out for additional thickness measurements of the Ion-Cut silicon layer. Figure 5-6 shows a cross-section image of the FOx layer capped handle wafer with the bonded exfoliated layer. The thickness of the exfoliated layer measured $1.418 \mu \mathrm{m}$.

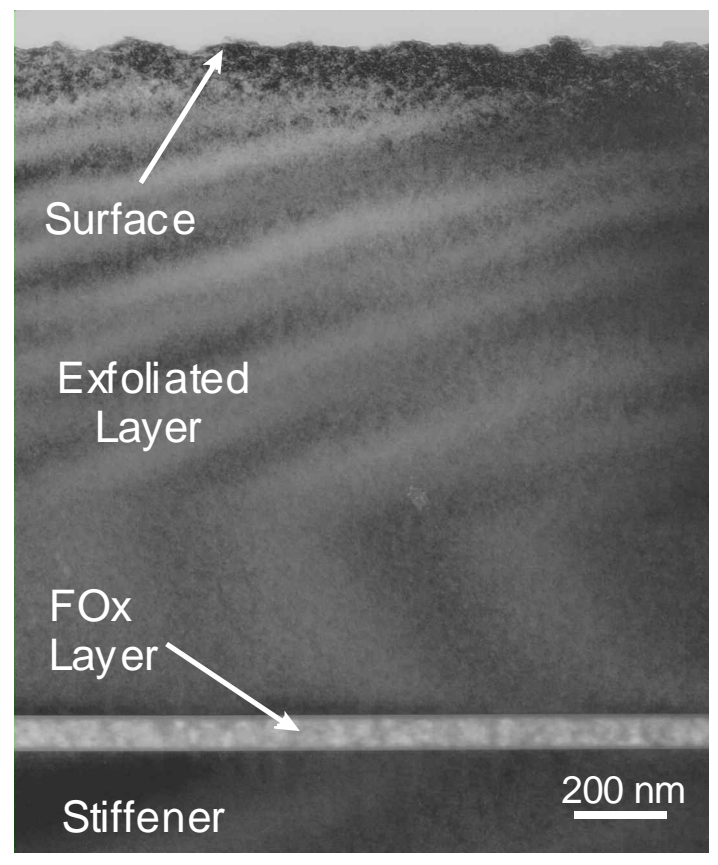

Figure 5-6: Low magnification cross-section TEM image of the hydrogen implanted silicon wafer after bonding and ion-cutting. 
Knowledge about the thickness of the exfoliated layer was also gained by AFM. Since the donor wafer was smaller in area size than the stiffener, AFM imaging could be carried out at the edge of the exfoliated layer and thus determining the height of the exfoliated layer was possible with this analysis method. Figure 5-7 shows the obtained AFM image of the edge of the exfoliated layer. The thickness of the exfoliated layer was evaluated by subtracting the surface height of the exfoliated layer, averaged over the whole imaged area from the height of the stiffener, also averaged over the scanned area. The average thickness of the exfoliated layer was measured to be $1.398 \mu \mathrm{m}$. This result is in very good agreement with the electron microscopy measurements.

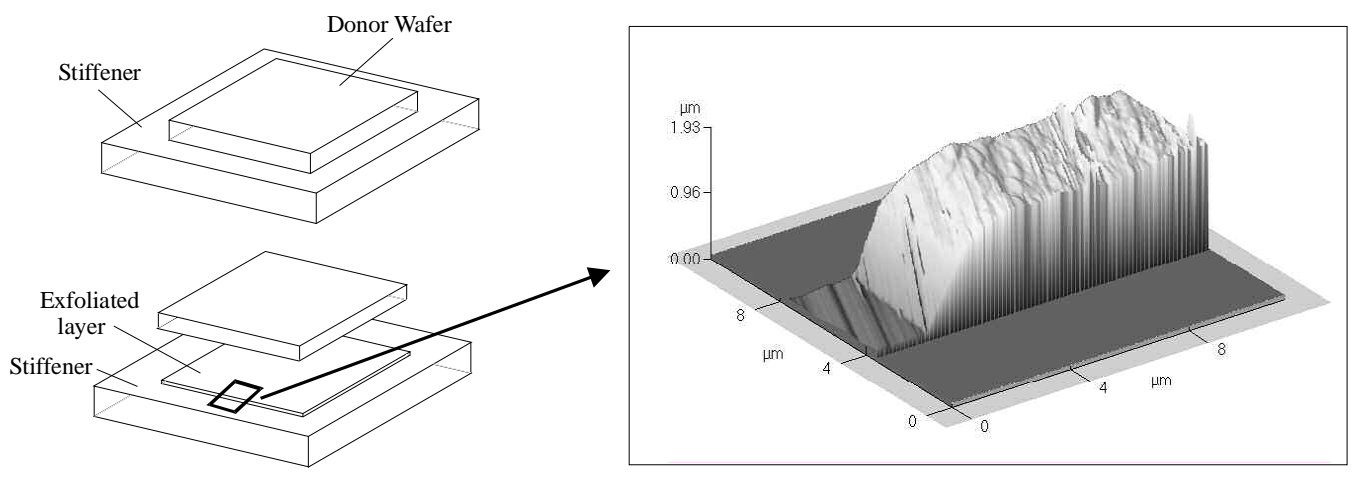

Figure 5-7: AFM image of the edge of the exfoliated layer

Summarizing the Ion-Cut depth measurements, different methods led to the following values for the average thickness of the exfoliated layer:

$\begin{array}{ll}\text { RBS channeling: } & 1.39 \mu \mathrm{m} \\ \text { XSEM: } & 1.416 \mu \mathrm{m} \\ \text { XTEM: } & 1.418 \mu \mathrm{m} \\ \text { AFM : } & 1.398 \mu \mathrm{m}\end{array}$

The values determined with the different analysis techniques vary only by $2.0 \%$. The good agreement in the Ion-Cut depth measurements strengthens the postulation that the Ion-Cut takes place in the region of maximum damage. 


\subsubsection{Microstructure of the Implantation Zone}

To achieve more information about the hydrogen implantation damage, XTEM imaging of the sample in the as-implanted state and also after ion-cutting was performed. Figure 5-8 presents a XTEM image of the implanted region in the as-implanted sample, obtained in an off Bragg condition. The error in the magnification and consequently in the depth measurements is only about $1 \%$, as determined by TEM standards. Analysis of the image reveals a $0.29 \mu \mathrm{m}$ broad densely damaged region starting at a depth of $1.32 \mu \mathrm{m}$, followed by a broad tail region of less dense damage. The tail region of the implanted zone is composed of some dislocation loops $(12-20 \mathrm{~nm}$ diameter) or rounded strain centres showing Ashby-Brown contrast [117], indicating that defects are strain centers, likely to result from the growth of small defects clusters into dislocation loops. The detailed nature of these loops (vacancy or interstitial) was not determined.

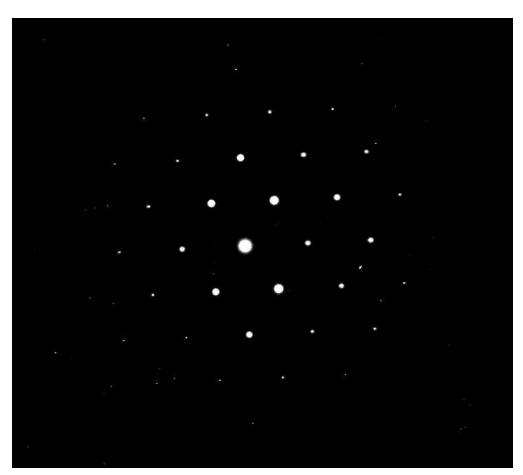

Figure 5-8: Low magnification cross section TEM bright field image and electron diffraction pattern of the hydrogen implanted silicon wafer in the as-implanted state. The electron diffraction pattern was obtained from the region above the densely damaged layer.

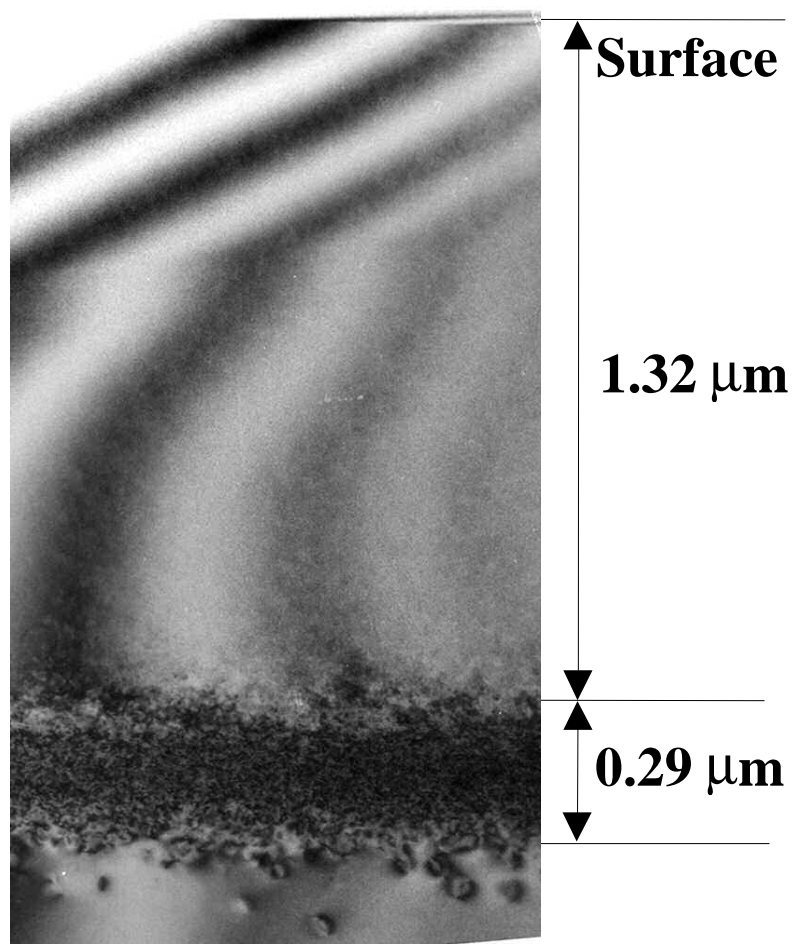

The electron diffraction pattern, taken from the low damaged region of the silicon over layer, reveals the high crystalline quality in this region. This is in accordance with the RBS channelling results.

To achieve more information from the implanted zone, XTEM images at higher magnification were obtained. Figure 5-9 (a) presents an image of the damaged region in the as-implanted material, obtained in an off Bragg condition. 


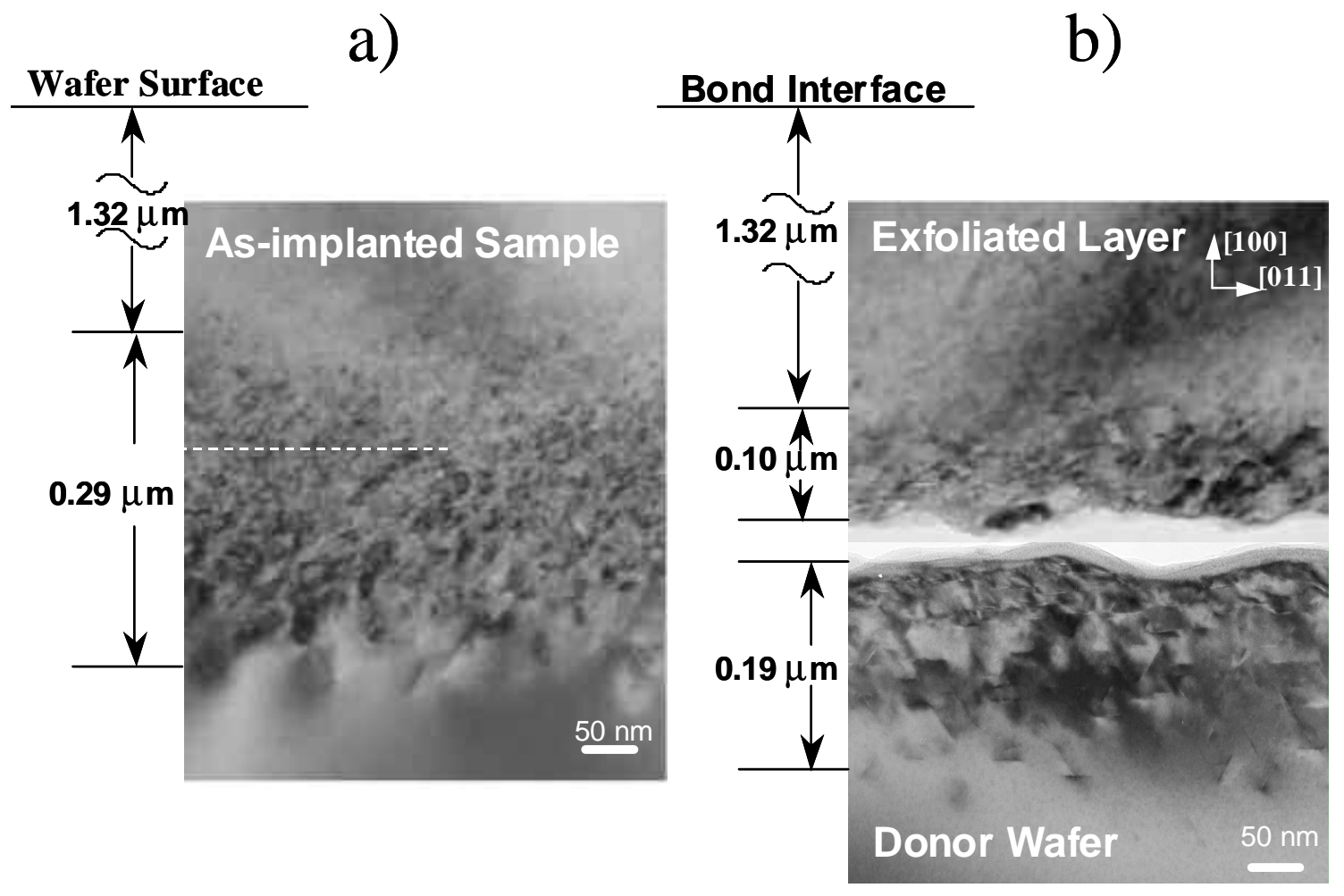

Figure 5-9: Bright field cross-section TEM images of the implantation zone in (a) the as-implanted sample and (b) after the Ion-Cut in the exfoliated layer and the donor wafer.

Closer to the surface small point defect clusters are visible, whereas below the implantation zone the silicon crystal appears to be undamaged. An analysis of the defects above the implantation damage zone at even higher magnification (not shown) revealed the point-like nature of these strain defects (vacancies or interstitials clusters). Upon annealing, higher resolution XTEM showed that these defects develop Ashby-Brown contrast [117]. That indicates, that these defects are equal in nature to the defects found in the tail region of the implantation zone (figure 5-8), i. e. dislocation loops, but smaller in size.

The damage region of the implantation zone (figure 5-9a) is composed mainly of planar defects. To illustrate that better, figure 5-10 shows a higher magnification XTEM image of the densely damaged region in the as-implanted sample. An analysis of the whole implantation damage depth region of the as-implanted sample shows that the majority of these cavities are aligned parallel to the surface in the (100) plane. However, about $20 \%$ of the platelets lie in the $\{111\}$ planes and appear throughout the region where the (100) platelets appear. Like others [9] I assume the planar defects visible in figures 5-9 (a) and 5-10 to be hydrogen-decorated platelets or small $\mathrm{H}_{2}$-gas bubbles. 
Figure 5-10: Higher resolution bright field XTEM image of the densely damage region of the as-implanted sample (see figure 5-9 (a))

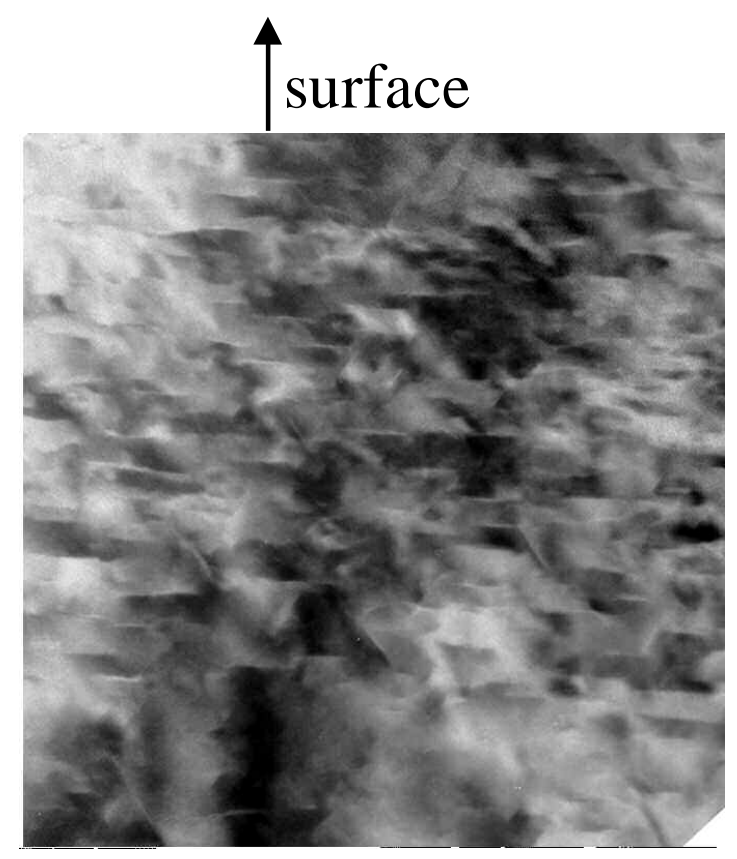

Figure 5-11 shows schematically the development of the $\mathrm{H}$-implant into $\mathrm{H}_{2}$-gas bubbles. Neglecting the chemistry of the hydrogen implant in silicon, the $\{111\}$ planes in the silicon crystal are known to be the energetically more favorable planes for cleavage. [118] But the chemical interaction between the implanted hydrogen and the silicon lowers the energy required to cleave $\{100\}$ planes in the silicon crystal. [119] In silicon, implanted hydrogen is known to be trapped at various implantation induced lattice defects like vacancies or silicon interstitials, passivating the dangling silicon bonds. [35] A common location for hydrogen atoms in p-type Si is the bond-centered

- Silicon

- Hydrogen

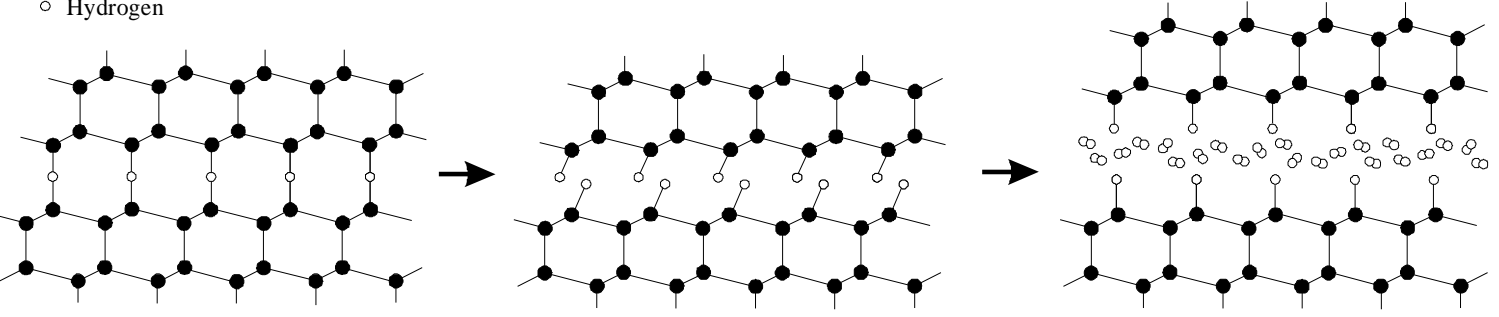

Figure 5-11: Schematic illustration of the $\mathrm{H}$-platelet formation and development into $\mathrm{H}_{2}$-gas bubbles. Hydrogen atoms reside at the bond centers of [111] Si-Si bonds; the Si-H-Si bonds are replaced by two Si$\mathrm{H}$ bonds, forming two $\mathrm{H}$-atom layers, only bonded by Van der Waals interaction. Indiffusing $\mathrm{H}$-atoms split the two $\mathrm{H}$-layers and agglomerate into a $\mathrm{H}_{2}$-gas. 
site. [120] The occupation of the bond centered sites in the silicon crystal by hydrogen results in the formations of $\{100\}$ H-planes. During annealing the single hydrogen atoms at the bond centered sites are replaced with two hydrogen atoms. In this state the atomic arrangement changes from Si-H-Si to Si-H-H-Si. [11]

Further in time or temperature, hydrogen atoms from other $\mathrm{Si}-\mathrm{H}$ defects in the system will diffuse to the $\mathrm{H}-\mathrm{H}$ platelets and agglomerate, forming regions of highpressurized $\mathrm{H}_{2}$-gas bubbles. [11,42] The high pressure in the $\mathrm{H}_{2}$-gas bubbles provide the force needed to generate a crack opening displacement, with cleavage occurring between the weakly bonded $\mathrm{H}-\mathrm{H}$ atoms.

It is believed that the $\mathrm{H}_{2}$ molecules that formed within these bubbles do so with a bond length almost equal the $\mathrm{H}_{2}$ bond length in vacuum, which leads to an energy gain that counterbalance the strain build up around the bubble in the silicon crystal. [42] Recent Raman spectroscopy measurements confirmed this assumption. [43] The vibrational frequency of the $\mathrm{H}_{2}$ molecules in the implantation zone was found to be equal to that of gaseous hydrogen. Due to their low surface energy the $\mathrm{H}-\mathrm{H}$ planes provide a favourable site for the formation and growth of such $\mathrm{H}_{2}$-gas bubbles.

During further heat treatment the $\mathrm{H}_{2}$-gas bubbles will grow in size via Ostwald ripening. [121] Finally, at a critical anneal duration or anneal temperature the H-bubbles coalesce and lead to the propagation of a crack through the whole silicon crystal. Figure 5-9 (b) shows XTEM images of the surface near regions of the newly cleaved surfaces after bonding and ion-cutting (see figure 5-1(d)). A comparison of figure 5-9 (a) and 5-9 (b) reveals that the platelets all have apparently the same thickness of about $1 \mathrm{~nm}$, either before or after annealing. But their length ranges between 5 and 15 $\mathrm{nm}$ in the as-implanted sample and between 15 and $30 \mathrm{~nm}$ after the Ion-Cut. This supports the belief that the platelets grow during annealing. Experimental confirmation of the process was provided by high-resolution XTEM studies. Detailed information before and after ion-cutting was obtained from higher magnification XTEM. Bright field off-Bragg images of the platelets, viewed edge-on in the [011] Si projection, are shown in figure 5-12. 

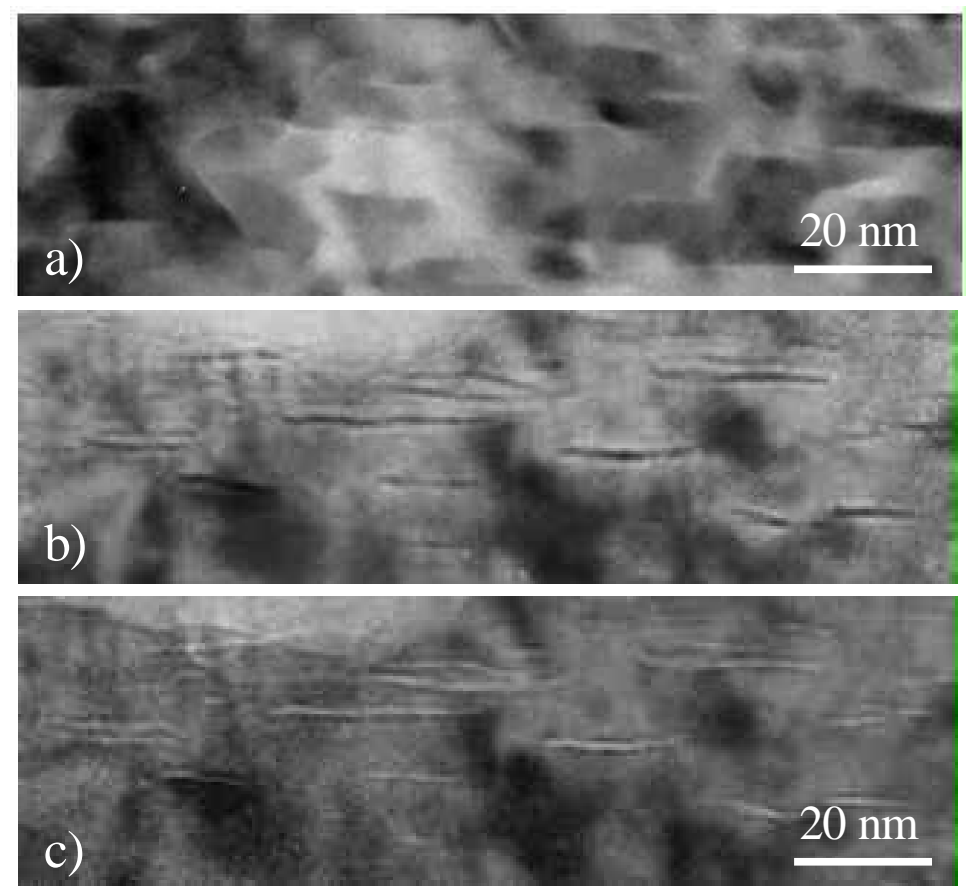

Figure 5-12: High magnification bright field XTEM images of the implantation damage zone in the asimplanted sample (a) and in the surface near region in the exfoliated layer after ion-cutting (b and c). The micro cavities are viewed in focus at the as-implanted sample (a) and in over focus (b) and under focus (c) at the exfoliated layer.

Figure 5-12 (a) presents an in focus image of the platelets in the as-implanted sample, whereas figure 5-12 (b) and (c) show the platelets after the Ion-Cut in the exfoliated layer. Figure 5-12 (b) was obtained from an over focus condition while the image in figure 5-12 (c) was obtained in under focus. The XTEM images confirm that the platelets all have the same thickness of about $1 \mathrm{~nm}$. Through focus imaging of the exfoliated layer reveals Fresnel contrast at the platelets in the cleaved samples: In over focus the micro cavities show dark Fresnel fringes, while in under focus they appear bright. This phase contrast is due to a change of the electronic potential at the platelets. Such a change in the electronic potential appears at planar voids or cavities, filled with a very low atomic number element (i. e. hydrogen). These data show that the planar defects are cavities or $\mathrm{H}$-precipitates rather than self-interstitials of Si. Through focus imaging of the platelets in the as-implanted sample did not show Fresnel contrast, indicating very little change in the electronic potential before annealing. Tilting about the axis normal to the plane of micro cavities revealed the 'platelet' rather than needle like morphology of these defects. The lack of Fresnel contrast at the platelets in the as-implanted sample and their smaller size compared to the platelets after ion-cutting suggests, that in the as-implanted state the platelets are less developed and contain lower amounts of hydrogen than the platelets observed after the Ion-Cut. However, the appearance of platelets or small $\mathrm{H}_{2}$-gas bubbles 
in the as-implanted sample indicates that beam heating occurred during the proton implantation process. The local temperature rise enhances short-range diffusion of hydrogen atoms and therefore could be the cause of platelet formation already taking place in the as-implanted state.

Re-examining figure 5-9 (b), the XTEM images show the cleavage surface to be rough at a microscopic scale. Some local changes from the (100) cleavage plane to $\{111\}$ planes are visible. An analysis of lower magnification XTEM images of the asimplanted sample and the exfoliated layer (not shown) show that the surface-to-platelet distance is the same for both and is measured to be $1.32 \mu \mathrm{m}$. Therefore, figure 5-9 (a) and (b) show the same depth region of the damaged silicon crystal. Thus, the depth in the as-implanted sample, where the cleavage in the Ion-Cut process will occur, could be determined and is marked by a dotted line in figure 5-9 (a). The XTEM images reveal, that the depth region where platelets appear does not change during the heat treatment and that the Ion-Cut occurs in the shallow part of the as-implanted platelet depth distribution. The damage zone is about $100 \mathrm{~nm}$ deep in the exfoliated layer, whereas it is measured to be about $190 \mathrm{~nm}$ deep, almost twice as thick, in the donor wafer. The distance from the bond interface to the cleavage plane (thickness of the exfoliated layer) was measured to be $1.42 \mu \mathrm{m}$.

Finally, it should be noted that previous research has shown, that the platelets visible in the XTEM images do not have a large influence on the direct backscattering yield in the RBS channeling spectra. [42] Hence, the lattice disorder detected by RBS channeling is not equal to the lattice disorder visible in the XTEM images. However, the contribution of the platelets to the direct backscattering yield has been shown to center at the peak region in the RBS channeling detected damage. [42] Therefore, the XTEM observations, which reveal that the Ion-Cut occurs in the shallower part of the platelet depth distribution, is in accordance with the RBS analysis results (chapter 5.1.1), which showed that the Ion-Cut takes place in the region of maximal damage or slightly shallower. 


\subsubsection{H-depth distribution before and after the Ion-Cut}

For a better understanding of the Ion-Cut process, it is also necessary to obtain information about the hydrogen depth distribution relative to damage depth distribution and the Ion-Cut location. Both theory and past experiment have shown that the damage profile resulting from ion implantation has a mean depth that is shorter than the ion projected range $[68,116]$ (see also chapt.2.3). Hence, when hydrogen is implanted into silicon at a constant implantation energy, the radiation damage associated with the proton implantation process is expected to peak somewhat shallower than the peak in the hydrogen concentration profile. However, it has been reported, that implanted hydrogen diffuses towards the damage peak if the implantation was performed at elevated temperatures. [122] More research is required to clarify, whether the implanted hydrogen, originally located deeper in the material than the implantation damage, diffuses towards the implantation damage during the heat treatment or if it remains at the depth it came to rest during the ion-implantation.

For the analysis of the hydrogen implantation distribution before and after ioncutting, ERD and SIMS measurement were performed. As in the RBS channeling analysis, ERD measurements were carried out not only on the as-implanted sample but also on samples with a slightly reduced implantation dose $\left(4.5 \times 10^{16} \mathrm{~cm}^{-2}\right)$, which was heat-treated but not bonded to a stiffener.

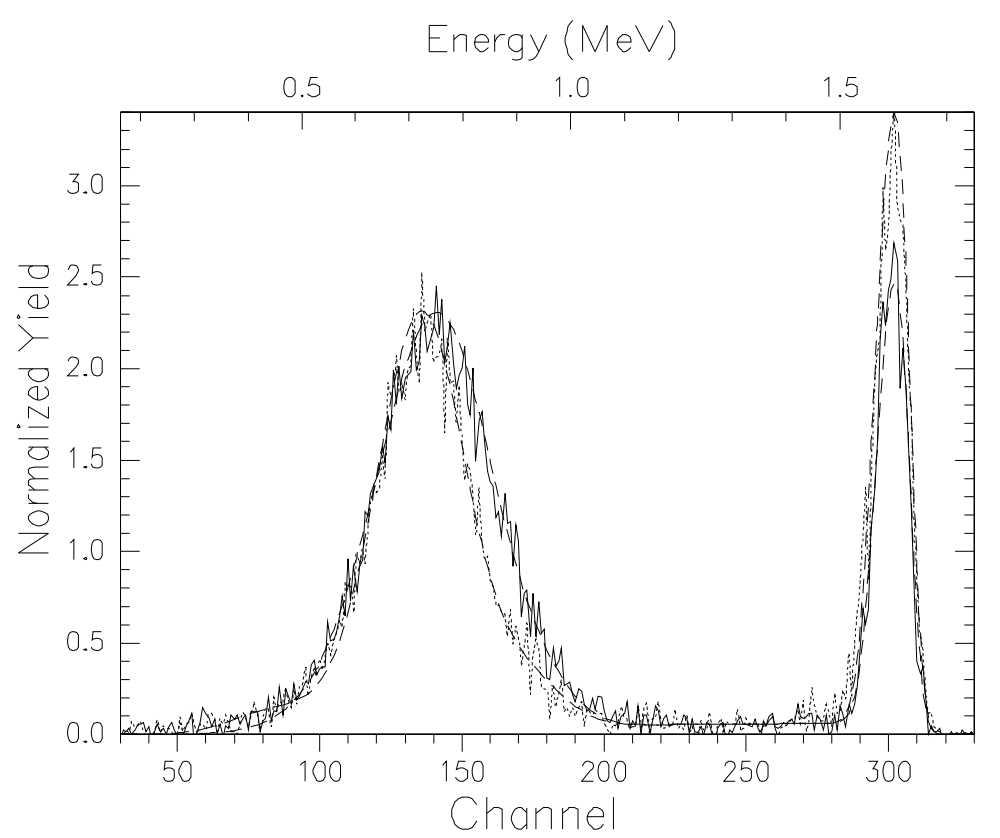

Figure 5-13: $4.3 \mathrm{MeV}$ ERD-spectra obtained from a silicon wafer implanted with $175 \mathrm{keV}$ hydrogen ions at a dose of $4.5 \times 10^{16} \mathrm{~cm}^{-2}$ in the as-implanted state (—) and after annealing for 3 hours at $200^{\circ} \mathrm{C}+5$ minutes at $600^{\circ} \mathrm{C}(\cdots \cdots)$. The simulations of the spectra are overlaid $(-\cdots--)$. 
Figure 5-13 shows the ERD spectra obtained from both samples. The peaks at high energy (channel 300) are caused by the hydrogen adsorbed at the sample surface, whereas the low energy peaks between channel 70 and 200 represent the concentration distribution of the implanted hydrogen, before and after annealing, respectively.

The ERD data were analyzed using the computer code RUMP. [84] As in the RBS channeling analysis, recent data for the stopping power of the ${ }^{4} \mathrm{He}^{2+}$ and also $\mathrm{H}^{+}$ ions in $\mathrm{Si}$ [81] were implemented in the calculation of the hydrogen depth distribution. For the evaluation of the total hydrogen content the ERD spectra were simulated using literature values of energy dependent cross sections for helium-hydrogen collisions, which apply to the experimental setup (scattering angle $=30^{\circ}$ ). [89] The simulations of the ERD spectra are also displayed in figure 5-13.

The results of the ERD analyses are presented in figure 5-14. It shows the hydrogen concentration in the sample as a function of sample depth. For comparison the implantation damage depth distributions, deduced from RBS channeling (figure 5-4) are overlaid. The vertical line represents the Ion-Cut locations determined by RBS

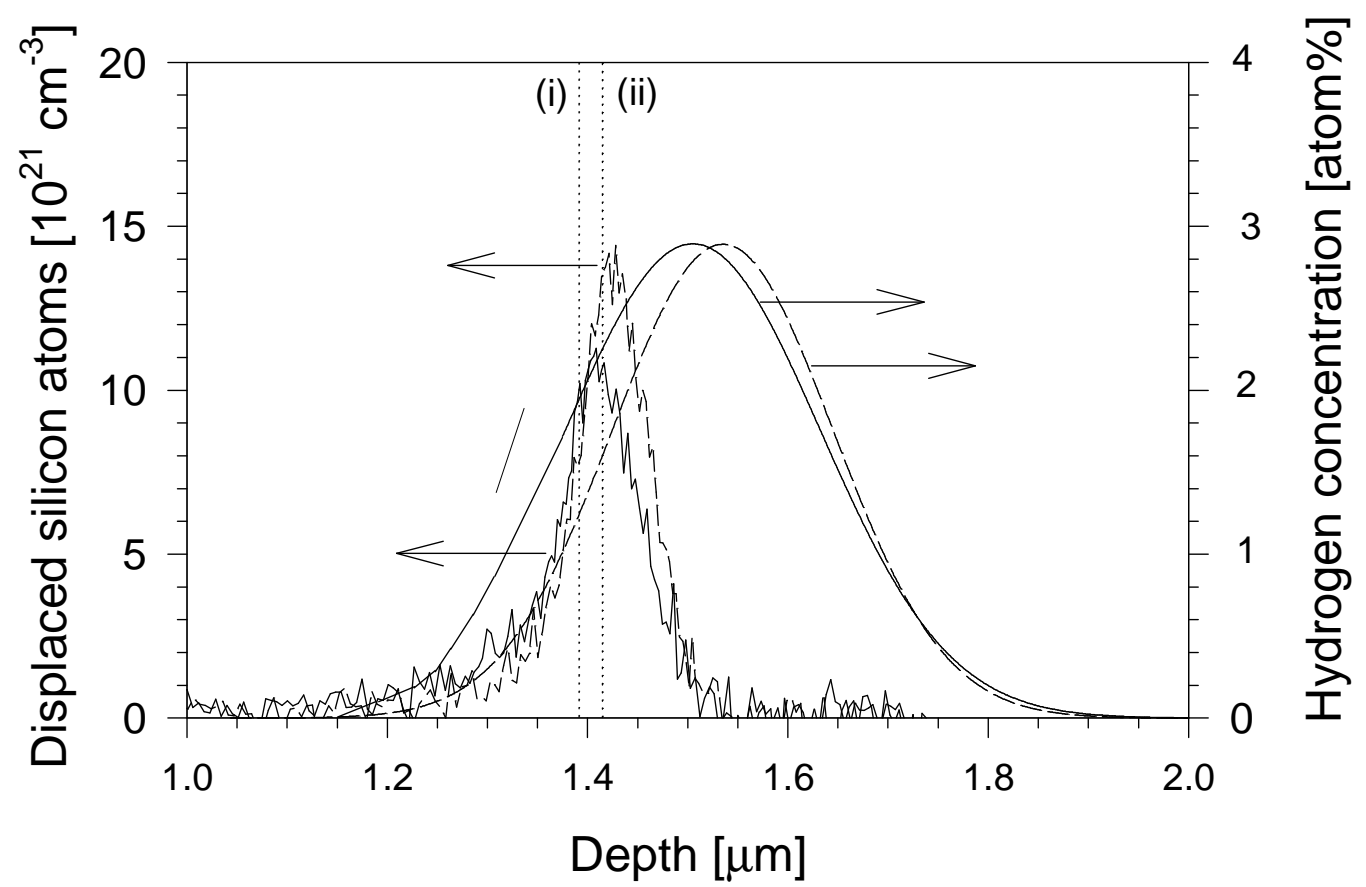

Figure 5-14: In-depth distribution of the implanted hydrogen in the as-implanted sample $(\longrightarrow)$ and after annealing for 3 hours at $200^{\circ} \mathrm{C}+5$ minutes at $600^{\circ} \mathrm{C}(--\longrightarrow)$. The hydrogen implantation dose was $4.5 \times 10^{16} \mathrm{~cm}^{-2}$. For comparison, the depth distribution of the displaced atoms in the as-implanted sample (—) and after annealing (- — - ), deduced from RBS channeling, are overlaid. The vertical dotted lines (i) and (ii) represent the depth interval, within which the Ion-Cut took place, as determined by RBS channeling, XSEM, XTEM, and AFM. 
channeling and SEM. The H-concentration in the as-implanted sample peaks at a depth of $1.505 \mu \mathrm{m}$, about $6 \%$ deeper than the implantation damage peak. The obtained ratio between the depths of the H-concentration peak and the implantation damage peak of 1.06 is consistent Monte Carlo simulations [75] and previous work on $\mathrm{H}$-implanted silicon at lower ion implantation energies [114]. Due to the energy straggling of the analyzing beam and the forward scattered hydrogen during the ERD measurement, the hydrogen distribution appears to be very broad. However, the results show clearly, that during the anneal the hydrogen located in the near surface region of the H-implantation distribution diffused out, whereas the hydrogen depth distribution remains unchanged at larger depths. The loss of hydrogen in the sample during annealing was evaluated to be $19 \%$ of the total hydrogen implantation dose. After the heat treatment the $\mathrm{H}$ concentration peak is located at a depth of $1.535 \mu \mathrm{m}, 30 \mathrm{~nm}$ deeper than in the asimplanted sample.

The diffusion coefficient of hydrogen in undamaged silicon at room temperature is known to be around $10^{-10} \mathrm{~cm}^{2} / \mathrm{sec}$. [123] and hence an appreciable broadening of the $\mathrm{H}$ implant distribution profile would be expected. However, the hydrogen resides at the implantation range and the shape of the hydrogen depth distribution is preserved at room temperature. That indicates implanted hydrogen is trapped in the silicon lattice. This result is consistent with previous research on hydrogen-implanted silicon [124] and earlier infrared spectroscopy studies identified these hydrogen traps to be various implantation induced lattice defects. [35]

The ERD spectrum of the annealed sample shows a partial out diffusion of hydrogen located in the shallower part of the H-depth distribution. The final step in the annealing process was carried out at $600^{\circ} \mathrm{C}$, a temperature high enough to cause the monatomic hydrogen to detrap from the lattice defects. [9] This anneal temperature leads to a high mobility of the free hydrogen atoms. The hydrogen out diffusion and internal platelet formation kinetics have been shown to be similar. [35] Therefore, fast moving atomic hydrogen near the surface can diffuse out.

In contrast to the surface near region of the hydrogen distribution, no macroscopic rearrangement of the H-implant at larger depths was detected. Hence, only short-range diffusion of the hydrogen atoms takes place after their liberation from lattice defects. The trapping of hydrogen at larger depths is consistent with previous research. [124] The data suggest that hydrogen at larger depths gets either trapped at the internal platelets or quickly diffuses into the vicinity of other atomic hydrogen due the large hydrogen concentration, forming slow moving $\mathrm{H}_{2}$ molecules. 
Weldon et al. showed by a combination of infrared spectroscopy and ERD that upon annealing to temperatures above $200^{\circ} \mathrm{C}$ an almost complete transformation of $\mathrm{Si}-\mathrm{H}$ complexes into $\mathrm{H}_{2}$ molecules occurs. [35] Hence, I assume that after annealing, $\mathrm{H}_{2}$ molecules comprise the bulk of the hydrogen signal in the ERD spectrum (figure 5-13). Theoretical investigations of hydrogen in silicon predict $\mathrm{H}_{2}$ molecules to be stable at tetrahedral interstitial sites. [125,126] But the solubility of $\mathrm{H}_{2}$ in silicon at room temperature is extremely small. [127] Therefore, I conclude that after the anneal the remaining hydrogen is trapped in form of $\mathrm{H}_{2}$ molecules in the $\mathrm{H}_{2}$-gas bubbles visible in the XTEM images (figure 5-11b and c) or other not yet identified traps in the silicon lattice.

The presence of the bond-interface in the Ion-Cut sample and its influence on the diffusion behavior of the $\mathrm{H}$-implant must be considered when drawing conclusions concerning the H-depth distribution in the Ion-Cut sample, based on the ERD results obtained from a non-bonded sample. Earlier research has suggested, that the sink strength of the bond interface to diffusing hydrogen is similar to the sink strength of a free surface. [35] Therefore, the change in the hydrogen distribution for the non-bonded sample and the bonded sample are expected to be the same.

Comparing the peak locations of the hydrogen concentration and the implantation damage in the annealed sample, the ion beam analyses reveal, that at the moment of Ion$\mathrm{Cut}$, the $\mathrm{H}$-concentration peak is about $100 \mathrm{~nm}$ deeper than the implantation damage and the Ion-Cut occurs at the region of highest implantation damage rather than at the $\mathrm{H}$ concentration peak. The ERD analysis also shows that the H-concentration at the Ion-Cut location is only about $70 \%$ of the $\mathrm{H}$ peak concentration.

Information about the hydrogen depth distribution was also obtained in the samples that underwent ion-cutting. The energy straggling in the ERD spectra makes it impossible to distinguish between the H-implant signal and the signal representing the surface contamination. Therefore, secondary ion mass spectroscopy (SIMS) was applied to monitor the hydrogen depth distribution after the Ion-Cut. The SIMS measurements allow the distinction between the signal from the hydrogen surface contamination and the signal from the hydrogen implant. The results of these measurements are presented in figure 5-15. It shows the hydrogen distribution in the exfoliated layer and the donor wafer as a function of sample depth. The large hydrogen signal from the silicon surface is removed in the plots and hence the signal shown in the SIMS spectra only represent subsurface hydrogen, i. e. the H-implant. The amount of hydrogen left in the exfoliated layer is $85 \%$ of that left in the donor wafer. This is consistent with earlier observation and conclusion that the Ion-Cut takes place above the depth of the H-concentration peak. 


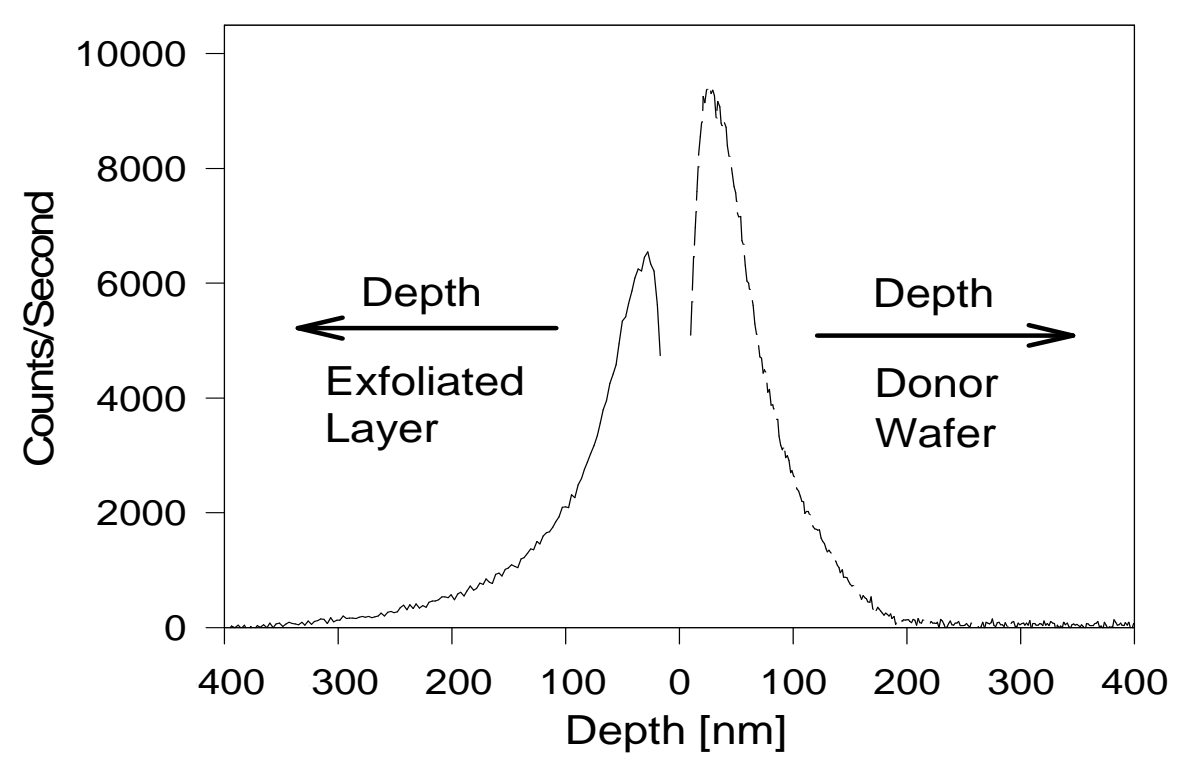

Figure 5-15: SIMS spectra of the hydrogen depth distribution after ion-cutting in the exfoliated layer and the donor wafer.

So far, the analyses of the depth distribution of the crystal damage (RBS channeling, XTEM), the hydrogen implant (ERD + SIMS), either before or after ion-cutting, and the location of the Ion-Cut (RBS channeling, XTEM and XSEM) show conclusively, that the Ion-Cut takes place at the location of highest crystal damage rather than at the depth of maximal hydrogen concentration.

The hydrogen implant was also monitored after the Ion-Cut by ERD, using a 2.2 $\mathrm{MeV}{ }^{4} \mathrm{He}^{2+}$ analyzing beam. The measurements were carried out to obtain quantitative information about the remaining amount of hydrogen in the sample. Figure 5-16 presents the ERD spectra taken from the exfoliated layer and the donor wafer. As mentioned earlier, the energy straggling in the ERD spectra prevents a distinction between the hydrogen from the H-implant and the hydrogen representing surface contamination. Thus, the information deduced from these two spectra provides an upper boundary for the total amount of hydrogen, remaining in the samples after the Ion-Cut $(\mathrm{H}-$ implant + surface contamination). 


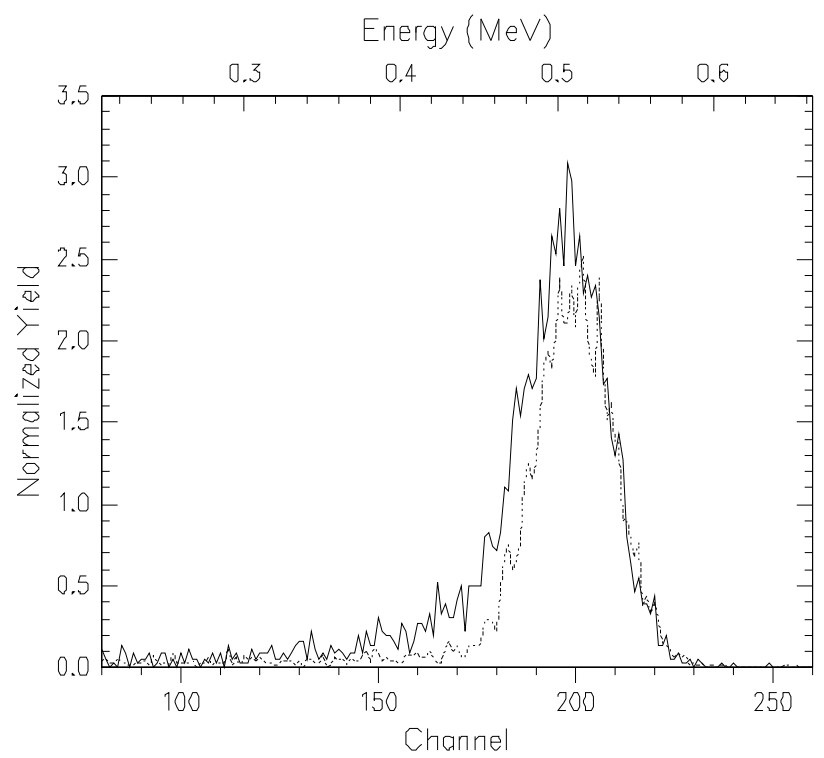

Figure 5-16: $2.2 \mathrm{MeV}$ ERD spectra of the exfoliated layer (dotted line) and the donor wafer (solid line) after ion-cutting.

The calculation gives a total $\mathrm{H}$-amount of $3.2 \times 10^{16}$ atoms $/ \mathrm{cm}^{2}$ in the exfoliated layer and the donor wafer. Although, this amount includes hydrogen from surface contaminations on both cleavage surfaces, it is lower than the implanted H-dose. This indicates that, even though the Ion-Cut didn't take place at the H-concentration peak, a large portion of the H-implant (at least 36\%) leaves the silicon crystal during the anneal and the Ion-Cut. It is unclear whether a significant amount of the vanished hydrogen participated in the cleavage process or left the sample by out diffusion after the Ion-Cut took place. However, the ERD analysis of the annealed, but not cleaved H-implanted silicon sample (see figure 5-13) revealed that about $20 \%$ of the H-implant leaves the implanted zone during annealing. Therefore at least another $16 \%$ of the implanted hydrogen leaves the sample during the Ion-Cut itself or later.

Previous research using in-citu mass spectroscopy experiments showed that about $30 \%$ of the H-implant is liberated in the moment the Ion-Cut takes place. [35] This is in accordance with above results considering that the hydrogen contaminations on both cleavage surfaces are included in the ERD dose measurements. The real loss of the Himplant is higher than measured (16\% of implantation dose), since the hydrogen surface contaminations, which amount is unknown, has to be added to the measured H-loss. 


\subsubsection{Influence of Stress and Strain Field on H-Platelet Formation and Ion-Cut Location}

To understand why Ion-Cut cleavage occurs near the peak in the damage distribution rather than the at the hydrogen concentration peak it is necessary to examine the mechanisms behind the $\mathrm{H}_{2}$-gas bubble nucleation and growth. As mentioned earlier, I assume the planar micro cavities detected in the XTEM images (Figure 5-9, 5-10, and 5-12) to be hydrogen formed platelets or gas bubbles. In p-type silicon, hydrogen atoms are reported to reside among other lattice sites in the bond-centered site [120], thus forming $\mathrm{H}$-planes parallel to $\{100\}$ planes of Si. The presence of such silicon hydride structures weakens the bonding between $\mathrm{Si}$ atoms and also passivates any dangling $\mathrm{Si}$ bonds, thereby reducing the surface energy needed to form two new (100) Si surfaces. Thus, the hydrogen reduces the fracture strength of Si.

However, to produce cleavage of the silicon crystal, the H-atoms must agglomerate to produce highly pressurized $\mathrm{H}_{2}$-gas bubbles, which provide the force for crack propagation. Hydrogen atoms located at the bond-centered site are the nuclei of such $\mathrm{H}_{2}$-gas bubbles (see figure 5-11). The region with the maximum concentration of hydrogen atoms residing on the bond-centered site will be the most probable location for $\mathrm{H}_{2}$-gas bubble formation and ultimate cleavage.

It has been observed in this work that the Ion-Cut takes place in the region of maximum damage. I assume that this occurs because of an out-of-plane tensile strain, which is caused by the proton implantation.

Hydrogen implantation produces defects in the substrate, which causes expansion of the silicon lattice throughout the implantation zone. Previous research has shown that radiation damage associated with ion implantation in silicon generates compressive biaxial stress at damage doses comparable to those found in Ion-Cut experiments. [128] It has been further shown in experiments that the stress scales with the defect concentration generated during ion irradiation. [129] Based on experiments [130] and modeling [131] on defects in $\mathrm{Si}$ the stress generating damage is most likely in the form of extended defects of defect clusters. Consequently, during the proton implantation compressive stress builds up in the silicon and the wafer develops a curvature, consistent with the observation of others (see also chapter 4.5). The change in curvature of the H-implanted sample due to the proton implantation was measured with a laser scanning setup (see chapter 4.5) and the corresponding integrated in-plane stress $S$ was calculated to be 447 $\mathrm{N} / \mathrm{m}$. 
In the presence of biaxial compressive stress there is also a component of tensile strain normal to the biaxial plane [132], i. e. in the present case normal to the silicon surface. This strain will also have a maximum at the location of maximum stress. It is my belief, that the out-of-plane tensile strain facilitates the incorporation of hydrogen atoms on the bond-centered sites between two neighboring silicon atoms by reducing the strain energy needed in forming the $\mathrm{Si}-\mathrm{H}-\mathrm{Si}$ bond in place of the $\mathrm{Si}-\mathrm{Si}$ bond. The $\mathrm{Si}-\mathrm{Si}$ bond length change for adding a $\mathrm{H}$ atom to the bond-centered site is significant. Theory predicts a change from the $\mathrm{Si}-\mathrm{Si}$ bond length of 2.35 Angstrom to a $\mathrm{Si}-\mathrm{H}_{\mathrm{BC}}-\mathrm{Si}$ bond length of 3.18 Angstrom. [31] Pre-straining the lattice with radiation damage results in a smaller length change upon $\mathrm{H}$ incorporation, thereby reducing the strain energy barrier to this process. Indeed, previous research has shown that the presence of this strain reduces the barrier to place $\mathrm{H}$ and $\mathrm{H}-\mathrm{H}$ between neighboring $\mathrm{Si}-\mathrm{Si}$ atoms, which lie along the $<111>$ direction. [133] This is further enhanced by the formation of a stable electronic bond between $\mathrm{H}$ and $\mathrm{Si}$. [120] Thus, the out-of-plane tensile strain enhances the development of H-platelets parallel to the surface, and therefore makes the location of highest out-of-plane tensile strain more favorable for platelets to nucleate.

Because the implantation damage and H-concentration are depth dependent the local stress field $\sigma(\mathrm{x})$ and the out-of-plane strain field are also depth dependent. The local stress field $\sigma(x)$ and the out-of-plane strain field are not only induced by the implantation damage alone, but also by the incorporation of the implanted hydrogen into the silicon lattice. Since the Si-Si bond length change for adding a $\mathrm{H}$ atom to the bondcentered site is significant the main contribution of the implanted $\mathrm{H}$ to the out-of-plane strain field is caused by the fraction of the H-atoms that occupies bond-centered sites between two neighboring $\mathrm{Si}$ atoms.

Infrared absorption spectroscopy showed, that although the bond-centered site is a common location for $\mathrm{H}$ atoms in crystalline $\mathrm{Si}$, the percentile of implanted $\mathrm{H}$-atoms residing at the bond-centered site, is quite low. [35] Exact values are to the best knowledge of the author not available at this time. To get an estimate of the depth of maximal out-of-plane tensile strain, the out-of-plane tensile strain contributions from hydrogen occupying the bond-centered sites and the implantation damage must be considered. Since the H-implant resides deeper in the material than the irradiation induced damage, an increase in the percentile of the $\mathrm{H}$-atoms, occupying the bondcentered sites results in a larger depth of maximum stress (see figure 5-17).

Therefore, the depth of maximum out-of-plane tensile strain was calculated as a function of the fraction of $\mathrm{H}$-atoms, residing on the bond-centered sites. The calculation 
were based on the $\mathrm{Si}-\mathrm{Si}$ bond length change caused by the incorporation of an $\mathrm{H}$ atom to the bond-centered site, predicted by theory to be 0.83 Angstrom. [31]

The calculation were facilitated by TRIM [75] derived damage and hydrogen distributions, which were modified to provide depth scales compatible with the data presented in figure 5-4 and 5-14.
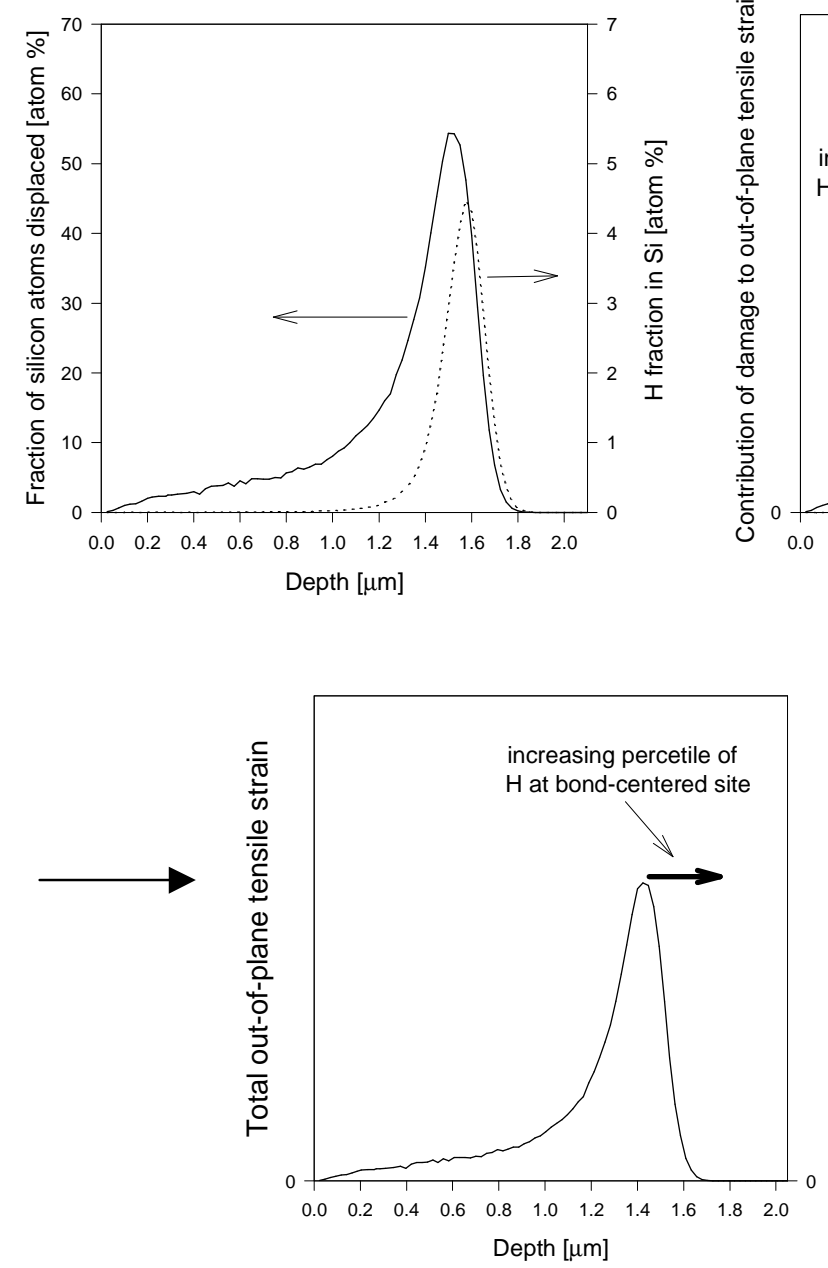

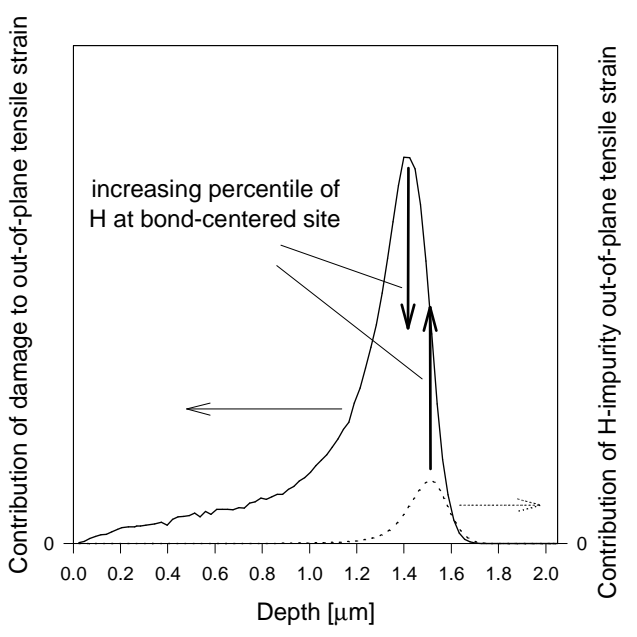

Figure 5-17: Influence of the occupation of the bond-centered site by $\mathrm{H}$ on the depth of maximum damage: Increasing percentile of $\mathrm{H}$ (located deeper than the damage) occupying the bond-centered sites leads to a shift of maximum damage towards larger depths.

The result of the strain calculations as a function of hydrogen occupation of the bondcentered sites is presented in figure 5-18. As can be seen, the depth of maximum out-ofplane tensile strain increases with increasing amount of $\mathrm{H}$ residing at the bond-centered site, as expected considering the increasing influence of $\mathrm{H}$ to the total out-of-plane tensile strain. It is expected that the amount of $\mathrm{H}_{\mathrm{BC}}$ will be less than $10 \%$ of the total $\mathrm{H}$ implantation dose, which is consistent with the $\mathrm{H}_{\mathrm{BC}}$ value by the strain value of $1.42 \mu \mathrm{m}$, 
the Ion-Cut depth determined in this work. This result suggests that radiation damage is the dominant component in determining the Ion-Cut location.

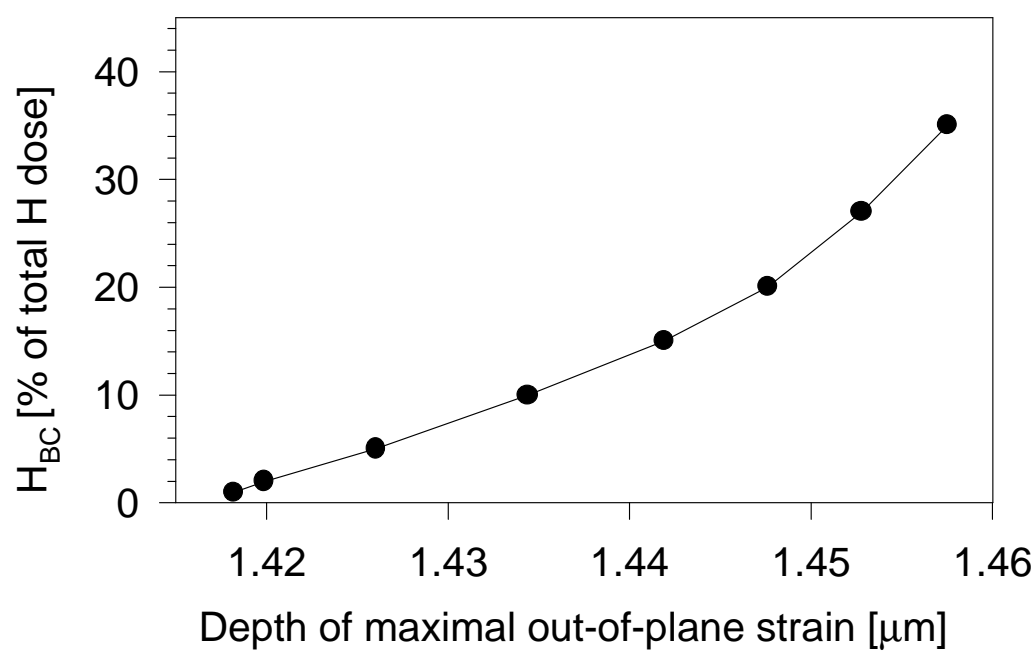

Figure 5-18: Calculated depth of maximum out-of-plane tensile strain as a function of H-implant percentile occupying the bond-centered sites.

This result is also consistent with earlier X-ray diffraction studies, which have shown that the out-of-plane strain in ion-implanted silicon is distributed along the damage distribution, with the maximum strain occurring at the damage peak [134,135].

I measured the depth distribution of the platelet density in the as-implanted sample as well as in the exfoliated layer and the donor wafer after ion-cutting. The data are deduced from TEM images similar to figure 5-9. To obtain better statistics the regions in which the platelets where counted were expanded to be five times as big as the sample areas shown in figure 5-9. The approach is semi quantitative, since in the calculation the assumption was made that the thickness of the TEM sample is constant within the regions in which the platelets were counted. In reality the TEM samples are wedge shaped. However, with the approximate approach the general trend of platelet nucleation as a function of depth is clearly visible.

Figure 5-19 presents the result of the platelet analysis. The platelet density in the as-implanted sample (upper part of figure 5-19) increases gradually from the depth they start to appear, reaching a maximum $1.42 \mu \mathrm{m}$ deep. Then, towards larger depths the platelet density decreases with a smaller rate up to the depth of $1.62 \mu \mathrm{m}$, at which it vanishes. This observation is in accordance with the proposed influence of the out-ofplane tensile strain on the platelet formation. At the depth of maximal out-of plane 
tensile strain, which is equal to the depth of maximum implantation damage (determined by RBS channeling to be $1.41 \mu \mathrm{m}$ ) the platelet density reaches its maximum.

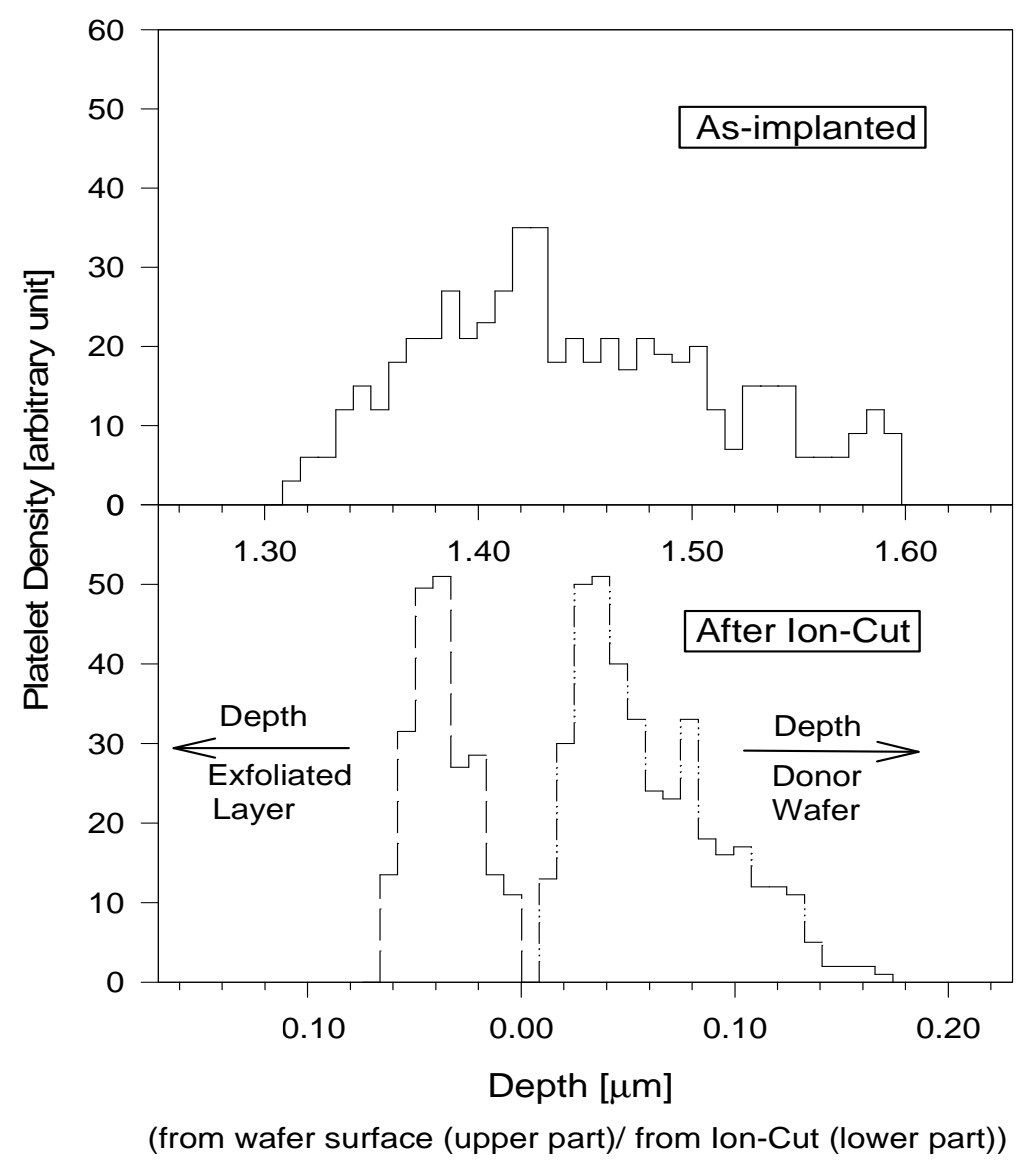

Figure 5-19: Depth distribution of the platelet density in the as-implanted sample ( $\longrightarrow$ ) (upper part) and also after ion-cutting in the exfoliated layer ( $-\longrightarrow$ ) and the donor wafer ( $\left.-{ }_{-}-\right)$(lower part). The data are deduced from XTEM images.

The lower part of figure 5-19 plots the depth distribution of the platelet density in the exfoliated layer and the donor wafer after ion-cutting. Due to the wedge shape of the TEM samples (see figure 4-27), their thickness decreases rapidly with decreasing distance to the cleavage surface. Therefore, also the number of detected platelets very close to the cleavage surfaces diminishes rapidly. In addition, all platelets $/ \mathrm{H}_{2}$-gas bubbles, which participated in the Ion-Cut form the new surfaces and hence cannot be seen anymore. In comparison to the as-implanted sample, the bell shape of the platelet density depth distribution is intensified after annealing. The peaks near the newly formed surfaces are much more pronounced than in the as-implanted sample. The fact that the platelet nucleation and growth are thermally activated suggests an increase of platelet 
density with annealing in the region of high implantation damage/out-of-plane tensile strain. Thus, the observed change in the platelet density distribution agrees with the proposed effect of the out-of-plane tensile strain on the platelet formation.

At the near surface and far surface side of the platelet depth distribution the platelet density decreases considerably. This confirms the Ostwald ripening process, by which the platelets grow and form highly pressurized $\mathrm{H}_{2}$-gas bubbles. The platelets in the region of high implantation damage grow at the cost of platelets located in the periphery of the platelet depth distribution.

Due to the significant importance of the implanted hydrogen in the Ion-Cut process, the general assumption was that the cleavage of the silicon crystal occurs at the hydrogen concentration peak. However, this work shows the nucleation and growth of hydrogen bubbles to take place at the peak in the damage/stress distribution rather than the peak of the hydrogen concentration distribution.

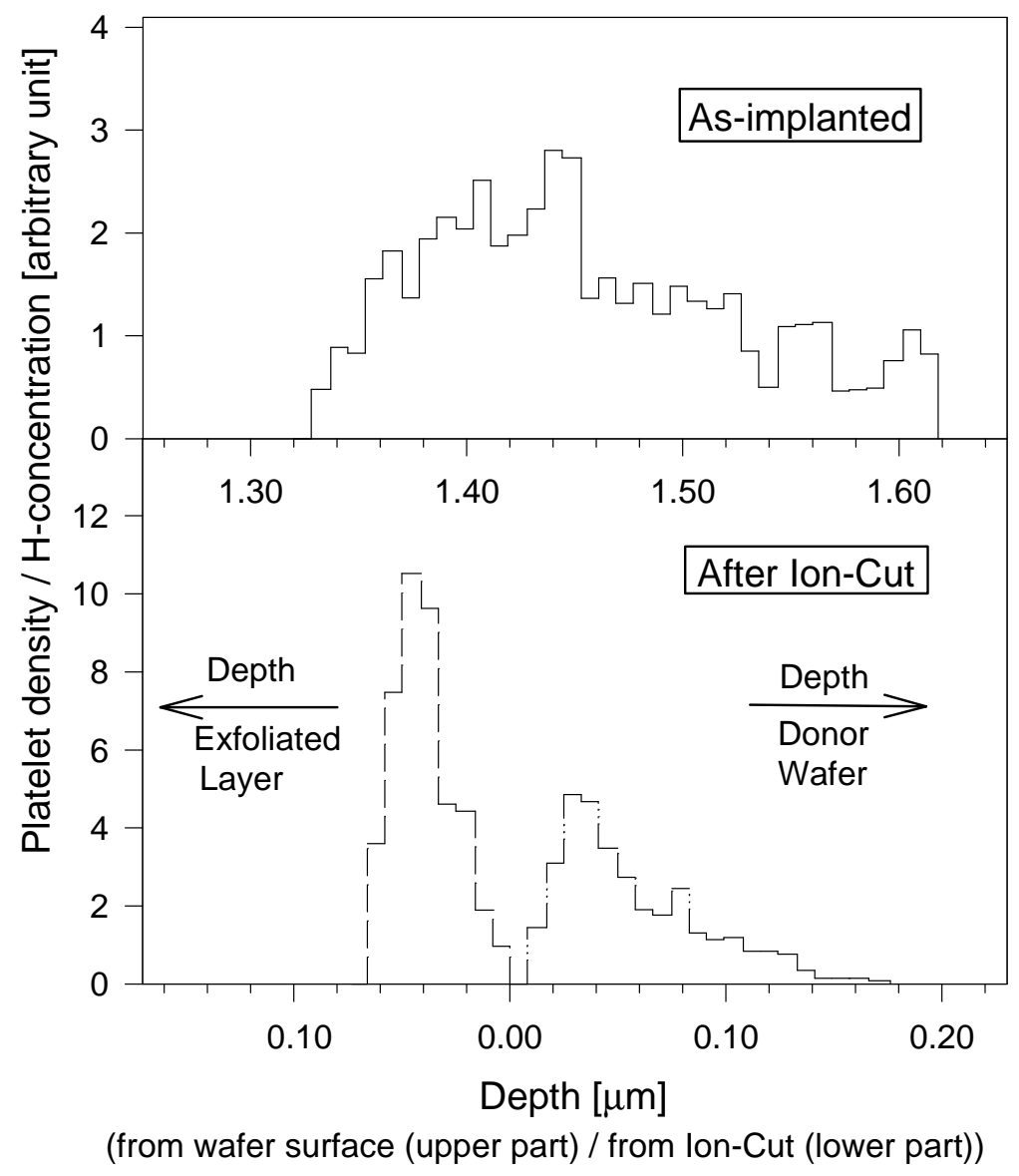

Figure 5-20: Depth distribution of the platelet density normalized to the hydrogen concentration depth distribution in the as-implanted sample (-) (upper part) and also after ion-cutting in the exfoliated layer ( $-\longrightarrow$ ) and the donor wafer (-- - - ) (lower part). 
To illustrate the crucial role of the implantation damage in terms of platelet formation and consequent ion-cutting, figure 5-20 plots the platelet density depth distribution normalized by the hydrogen concentration depth distribution as a function of depth. The normalized platelet distribution after ion-cutting was constructed using the $\mathrm{H}$ depth distribution, determined from the annealed but not cleaved sample (figure 5-14). Figure 5-20 illustrates the importance of the implantation damage in terms of H-platelet nucleation. It is clearly visible, that the efficiency of the $\mathrm{H}$-implant to nucleate $\mathrm{H}$ decorated platelets is considerable higher in the depth region of high implantation damage than it is in the depth region, where the H-concentration reaches its peak, but the implantation damage is low. 


\subsection{Influence of the Hydrogen Implantation Dose on the Ion-Cut}

The research presented in chapter 5.1 showed conclusively, that for H-implantation dose of $5 \times 10^{16} \mathrm{~cm}^{-2}$, the Ion-cut occurs at the depth of maximum damage rather than at the depth of maximum hydrogen concentration. These results were explained by the stress and strain fields that develop in implantation zone and the enhancing effects these fields have on $\mathrm{H}_{2}$-gas bubble nucleation.

When the hydrogen implantation dose increases, the stress and strain fields in the implantation dose will change. In addition, with increasing hydrogen implantation dose, the fracture toughness of the material will also be influenced by the large amount of ion irradiation induced damage accumulation within the implantation zone. Consequently, at higher implantation doses, the physical mechanisms that control the Ion-Cut process and the cleavage location might change, too.

Knowledge about the dependence of the stress field in the samples as a function of implantation dose will be crucial for the interpretation of how the Ion-Cut process changes with dose. Hence, for these experiments the silicon wafers were implanted with various hydrogen implantation doses, covering a wide range from $1 \times 10^{16} \mathrm{~cm}^{-2}$ to $1 \times 10^{17} \mathrm{~cm}^{-2}$. The substrates used in this experiment were $\langle 100\rangle$ oriented p-type silicon wafers (boron doped) with a resistivity of 1-10 $\Omega \mathrm{cm}$. The samples were implanted with $42 \mathrm{keV}$ protons to doses of $1,3,5,7 \times 10^{16} \mathrm{~cm}^{-2}$, and $1 \times 10^{17} \mathrm{~cm}^{-2}$, respectively. All ion implants were performed at liquid nitrogen temperature. To minimize channeling effects, the incident ion beam was oriented at 7 degrees from the surface normal.

Following the ion implantation, the samples were annealed without prior bonding to another substrate. The heat treatment was carried out in vacuum at $600{ }^{\circ} \mathrm{C}$ for 30 minutes. Since the H-implanted samples were not bonded to a stiffener, cleavage of the whole wafer does not occur. Instead, individual subsurface $\mathrm{H}_{2}$-gas bubbles, 3 to $10 \mu \mathrm{m}$ in diameter, form. The high internal pressure of these bubbles provokes the delamination of the silicon over layer in some of the bubbles, resulting in a blistered surface appearance. (see figure 5-21) 
With bonded wafer as stiffener

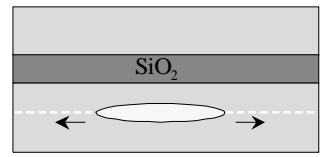

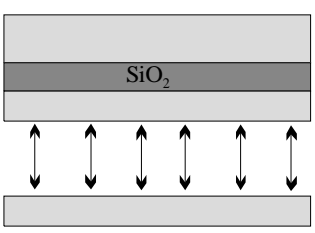

Figure 5-21: Illustration of surface blister formation
Without bonded wafer

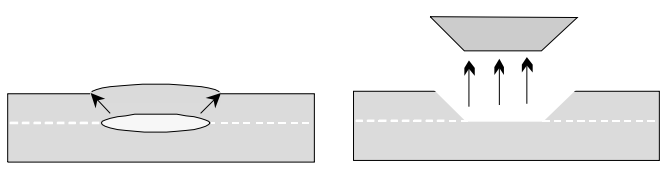

Previous research has shown that the blister depth for non-bonded samples is equal to the Ion-Cut depth for bonded samples. [31] The high anneal temperature and long anneal duration were chosen to ensure intense surface blistering. The base vacuum in the annealing treatment was $5 \times 10^{-8}$ Torr.

The implantation damage and hydrogen depth distribution were evaluated with ion beam techniques (RBS Channeling and ERD). The surface blister depths were measured by XSEM, AFM, and XTEM imaging. Knowledge about the microstructure was obtained by XTEM.

\subsubsection{Implantation Damage and Hydrogen Depth Profiles in the As-Implanted State}

Channeling RBS was applied to achieve knowledge of the crystalline quality as a function of depth of the as-implanted silicon samples. Figure 5-22 presents the ion channeling backscattering spectra obtained from the as-implanted samples. For comparison a spectrum in channeling condition and a random spectrum of a virgin silicon are also shown.

The peaks in the spectra of the as-implanted sample located between channel 325 and 375 indicate lattice damage induced by the hydrogen implantation. The backscattering yields $\chi_{\min }$ of the as-implanted samples at higher channel numbers (375$425)$ are quite low even for the highest implantation dose of $1 \times 10^{17} \mathrm{~cm}^{-2}\left(\chi_{\min }<9 \%\right.$ of the random signal). 


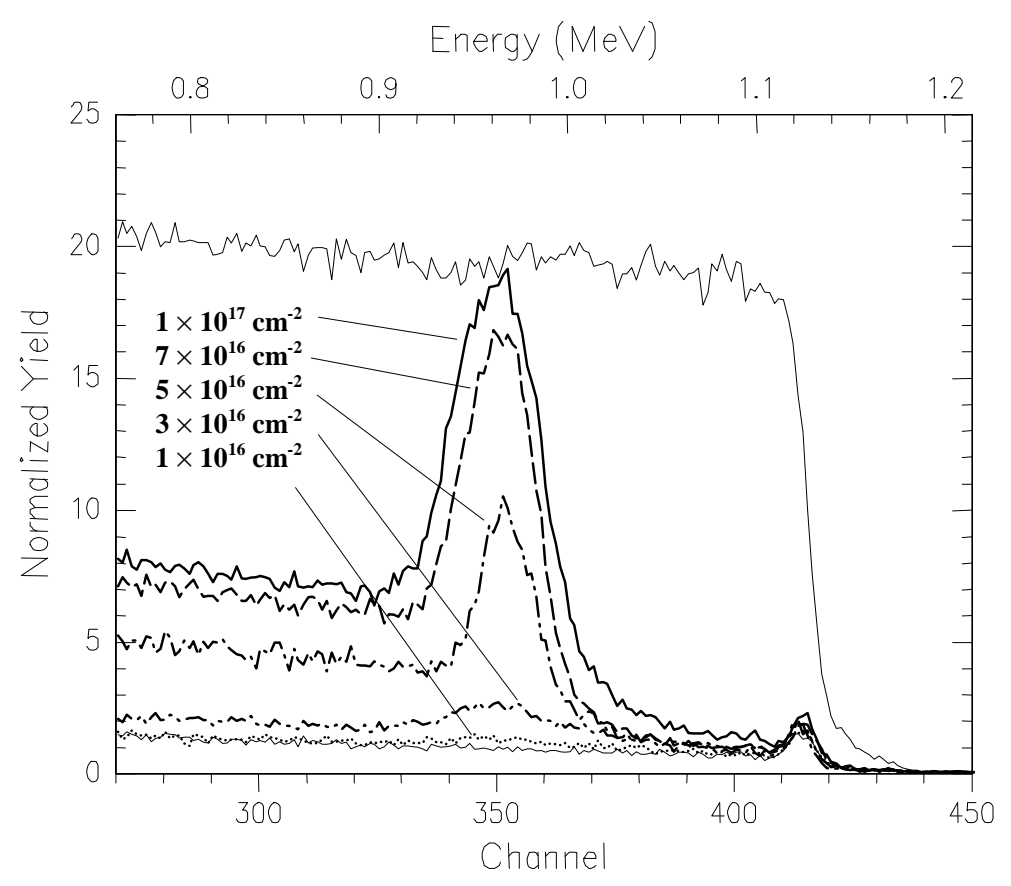

Figure 5-22: $2.0 \mathrm{MeV}$ He RBS-channeling spectra of the $\mathrm{H}$-implanted $\langle 100\rangle \mathrm{Si}$ in the as-implanted state. The H-implantation doses are $1 \times 10^{16} \mathrm{~cm}^{-2}(\ldots \cdots), 3 \times 10^{16} \mathrm{~cm}^{-2}(-\cdots-\cdots-), 5 \times 10^{16} \mathrm{~cm}^{-2}$ $(-\cdot-\cdot-), 7 \times 10^{16} \mathrm{~cm}^{-2}(--\longrightarrow)$, and $1 \times 10^{17} \mathrm{~cm}^{-2}$ ( $\longrightarrow$ ). Also shown for comparison are a random spectrum and the channeling spectrum of untreated silicon (thin solid lines).

For the investigation of the dependence of the ion-cut location on the hydrogen implantation dose, it is essential to obtain high accuracy in the damage depth distribution. Therefore, the depth distribution of the implantation damage in the as-implanted sample were determined from the obtained channeling data by the analyzing method described in chapter 4.2.1. The value of $\alpha\left(S_{\text {channeling }}=\alpha \times S_{\text {random }}\right)$ was 0.62 at the surface (channel 425 ) and increased up to 0.85 at channel 320.

Since ion-cutting was achieved only for the 3 highest implantation doses, the analyses was performed only on the sample implanted to doses of 5, 7, and $10 \times 10^{16} \mathrm{H} / \mathrm{cm}^{2}$. The results of the RBS channeling analysis are shown in figure 5-23.

The damage data determined from channeling measurements reveal that the implantation damage peaks at a depth of $410 \mathrm{~nm}$, independent of the implantation dose. 


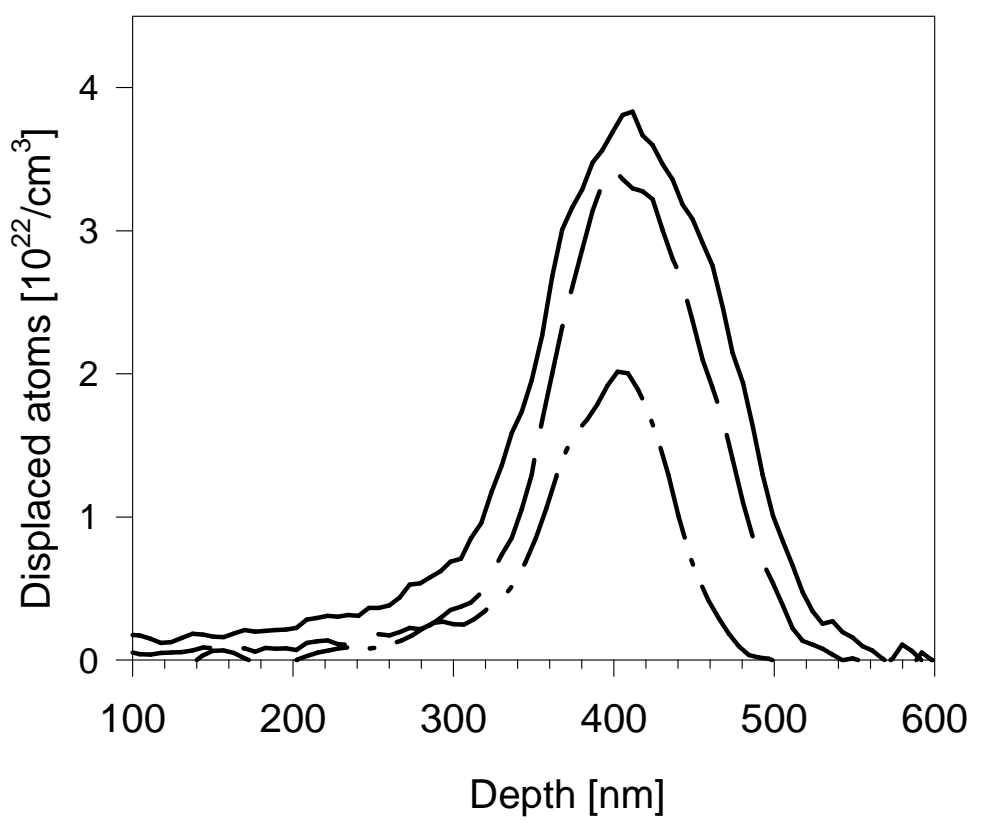

Figure 5-23: In-depth distribution of the implantation damage concentration. The H-implantation doses are $5 \times 10^{16} \mathrm{~cm}^{-2}(-\cdot-\cdot-), 7 \times 10^{16} \mathrm{~cm}^{-2}(--\longrightarrow)$, and $1 \times 10^{17} \mathrm{~cm}^{-2}(-)$.

The defect concentration in the silicon samples varies with varying hydrogen implantation dose. The RBS channeling analysis reveals that the implantation damage does not scale linearly with the implantation dose. The amount of displaced silicon atoms for the sample implanted to a $\mathrm{H}$-dose of $7 \times 10^{16} \mathrm{~cm}^{-2}$ is considerably larger than the one in the sample implanted to a H-dose of $5 \times 10^{16} \mathrm{~cm}^{-2}$. This is due to the interaction of the $\mathrm{H}$-implant with the ion irradiation induced lattice defects. The H-atoms decorate vacancies and interstitials, thus preventing Frenkel pair recombination, leading to a larger amount of residual lattice damage. This effect is indicated also in the raw RBS channeling data (figure 5-22). The samples implanted with $1 \times 10^{16} \mathrm{H} / \mathrm{cm}^{2}$ and $3 \times 10^{16} \mathrm{H} / \mathrm{cm}^{2}$, respectively, show very low direct backscattering yields compared to samples with higher H-implantation doses.

ERD was employed to gain knowledge about the H-concentration depth profiles. Figure 5-24 shows the ERD spectra obtained from the as-implanted samples. Since the Hprofiles of the samples implanted to the two lower doses did not produce an effect of interest, only the spectra for the $5,7 \times 10^{16} \mathrm{H} / \mathrm{cm}^{2}$, and $1 \times 10^{17} \mathrm{H} / \mathrm{cm}^{2}$ samples are plotted. The peak at high energy (channel 205) is caused by the hydrogen surface contamination, whereas the low energy peaks between channel 120 and 180 represent the concentration distributions of the implanted hydrogen. 


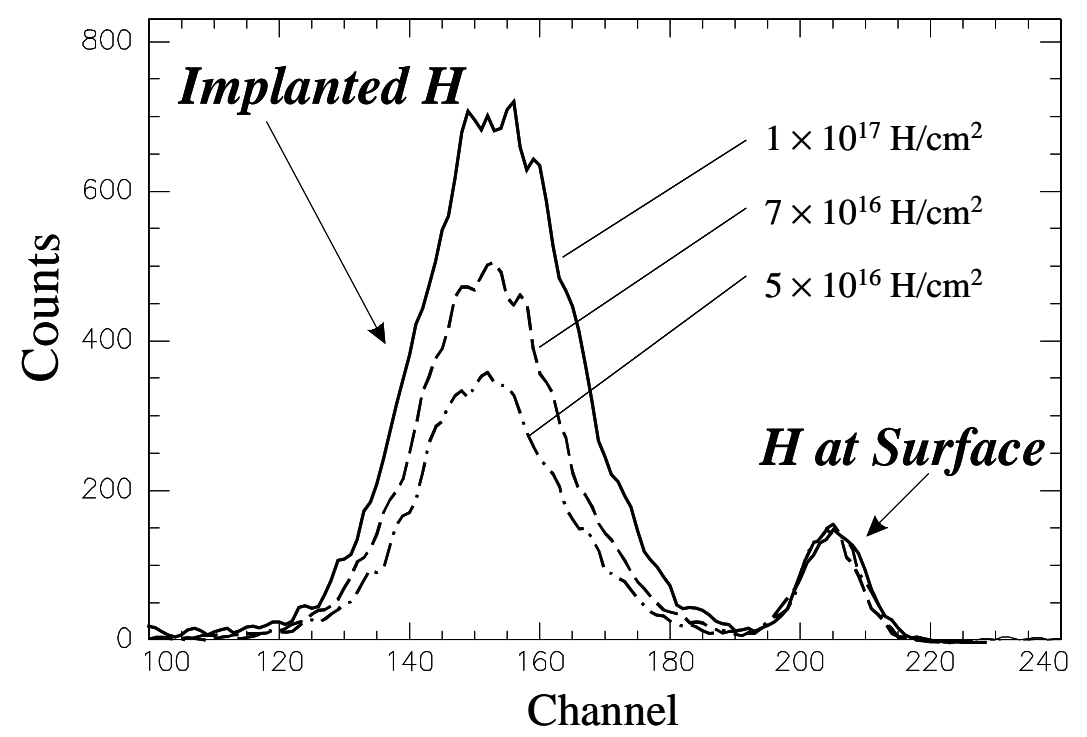

Figure 5-24: $3.0 \mathrm{MeV}$ He ERD spectra of the $\mathrm{H}$-implanted $\langle 100\rangle \mathrm{Si}$ in the as-implanted state. The $\mathrm{H}-$ implantation doses are $5 \times 10^{16} \mathrm{~cm}^{-2}(-\cdot-\cdot-), 7 \times 10^{16} \mathrm{~cm}^{-2}(-\square-)$, and $1 \times 10^{17} \mathrm{~cm}^{-2}(\longrightarrow$ ).

As for the implantation damage, high accuracy of the H-concentration depth profile is crucial for the examination of the Ion-Cut process. Therefore, the ERD data were analyzed using the channel-to-depth conversion and the energy spread correction described in chapter 4.1.2. The results of the ERD data analysis are presented in figure $5-25$.

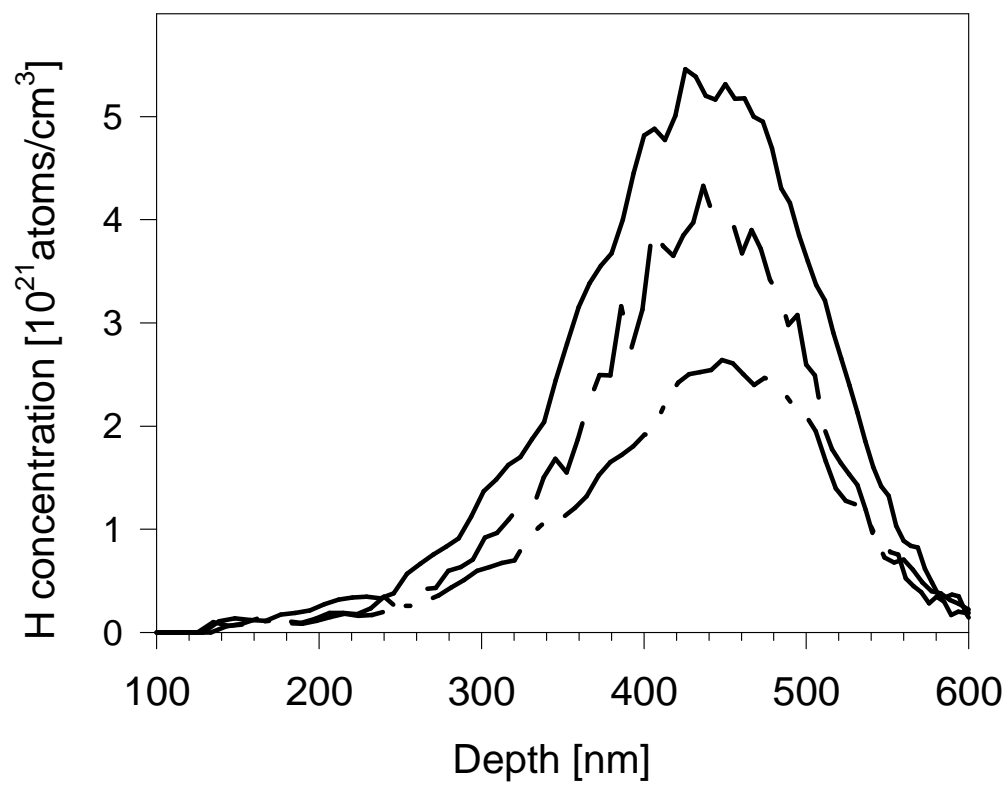

Figure 5-25: In-depth distribution of the as-implanted hydrogen concentration. The H-implantation doses are $5 \times 10^{16} \mathrm{~cm}^{-2}(-\cdot-\cdot-), 7 \times 10^{16} \mathrm{~cm}^{-2}(--\longrightarrow)$, and $1 \times 10^{17} \mathrm{~cm}^{-2}(-)$. 
The shape of the H-concentration depth profiles is the same for all implantation doses. Similar to the implantation damage, the H-concentrations peak at the same depth independent of the H-dose. The H-peak depth was measured to be $445 \mathrm{~nm}$, about $8.5 \%$ deeper than the implantation damage peak. This is consistent with both theory and past experiment, which show that the damage profile resulting from ion implantation has a mean depth that is shorter than the ion projected range. [68,116] Furthermore, TRIM calculations [75] showed a damage depth distribution peaking $8.3 \%$ shallower than the $\mathrm{H}$-concentration depth distribution. This is in very good agreement with the experimentally obtained results.

\subsubsection{Microstructure after the Heat Treatment}

Following ion beam characterization the implanted samples were annealed at $600{ }^{\circ} \mathrm{C}$ for 30 minutes and then further examined by plane view and cross-section SEM, AFM, and XTEM.

As mentioned earlier, the silicon samples, implanted with $\mathrm{H}$-ions to doses of $1 \times 10^{16} \mathrm{~cm}^{-2}$ and $3 \times 10^{16} \mathrm{~cm}^{-2}$ did not develop surface blisters during the anneal. This is consistent with theoretical calculations [10], which predict that a minimum amount of $\mathrm{H}$ dose is required for the pressure buildup within the $\mathrm{H}_{2}$-gas bubbles to induce ion-cutting. If the $\mathrm{H}$-concentration is too low and the anneal temperature high enough to cause detrapping of the $\mathrm{H}$-atoms from the various $\mathrm{H}$-complexes, as in this case, the hydrogen will diffuse out of the implantation zone without causing cleavage of the silicon wafer. Since only about $1 / 3$ of the total H-content in the sample participates in the Ion-Cut [35], the two lowest implantation doses in these experiments turn out to be insufficient for the development of surface blisters.

However, for the three highest implantation doses, very intense surface blistering, i. e. a high densities of surface blisters per unit area, was achieved. Figure 5-26 shows plane view SEM images of these sample surfaces. Clearly visible are the craters, left 

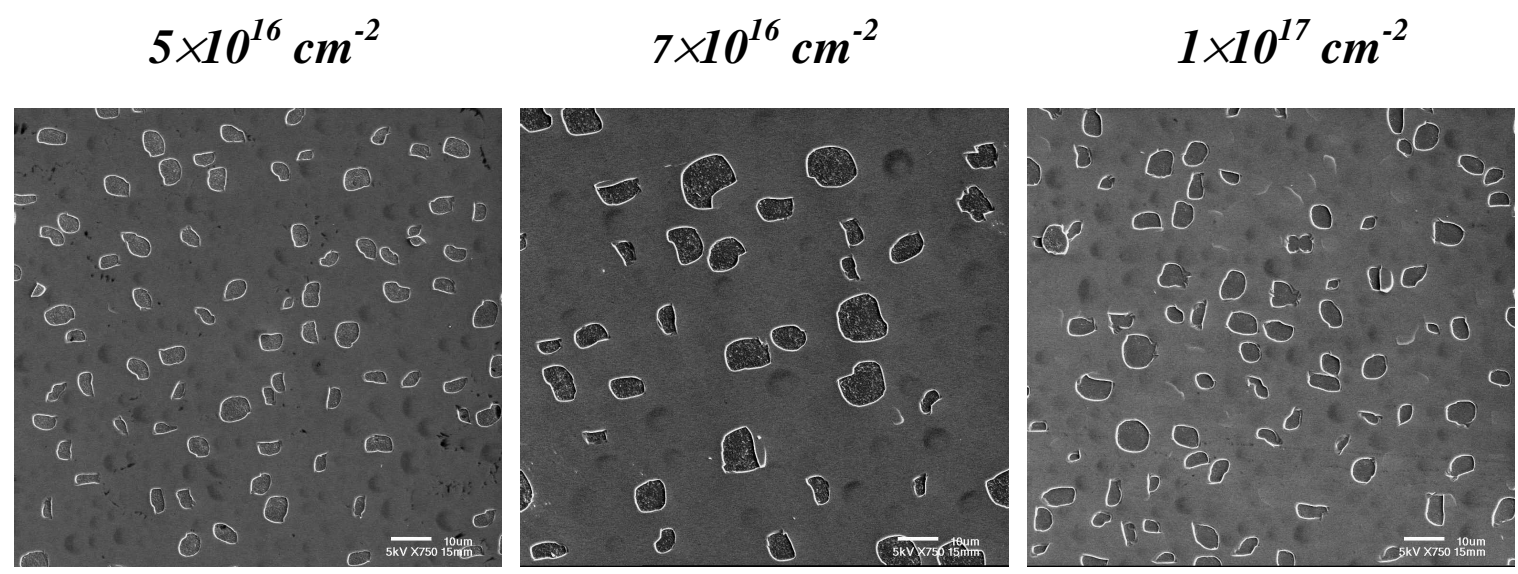

Figure 5-26: SEM plane view images of the blistered sample surfaces after H-implantations to different doses and annealing at $600{ }^{\circ} \mathrm{C}$ for 30 minutes.

behind after the silicon over layer exfoliated. Also observable are extrusions on the sample surface, caused by sub-surface hydrogen bubbles, which do not have the required pressure to facilitate the exfoliation of the silicon over layer.

XSEM imaging of the annealed samples was performed to determine the depth location where surface blistering occurred. Figure 5-27 presents plane view and cross-section SEM images of a surface blister of each sample. For the samples implanted to H-doses of $5 \times 10^{16} \mathrm{~cm}^{-2}$ and $1 \times 10^{17} \mathrm{~cm}^{-2}$, the XSEM imaging reveals a very uniform depth of the surface blister and consequently a very smooth cut of the H-implanted silicon crystal. The XSEM determined average depth location of surface blistering was measured to be $418 \mathrm{~nm}$ for the sample with an implantation dose of $5 \times 10^{16} \mathrm{~cm}^{-2}$, and $457 \mathrm{~nm}$ for the sample, implanted to a dose of $1 \times 10^{17} \mathrm{~cm}^{-2}$. Interestingly, the morphology of the blister bottom that results from the intermediate implantation dose of $7 \times 10^{16} \mathrm{H} / \mathrm{cm}^{2}$ shows that the blister depth oscillates between two distinct depths where ion-cutting occurred. The shallower ion-cut location has an average depth of $418 \mathrm{~nm}$, equal to the blister depth in the sample with an implantation dose of $5 \times 10^{16} \mathrm{~cm}^{-2}$. The deeper ion-cut took place at an average depth of $456 \mathrm{~nm}$, roughly equal to the blister depth in the sample with an implantation dose of $1 \times 10^{17} \mathrm{~cm}^{-2}$. This oscillation in Ion-Cut depth can also be seen in the plane view image of the surface blister.

Examining the plane view SEM images of the surface blister for the samples with an implantation dose of $5 \times 10^{16} \mathrm{~cm}^{-2}$ and $1 \times 10^{17} \mathrm{~cm}^{-2}$, the roughness of the blister bottom and thus the roughness of the Ion-Cut appears to be larger for the lower H-dose implant. 


\section{plane view}

a)

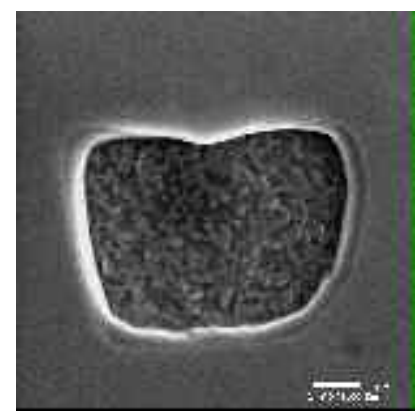

b)

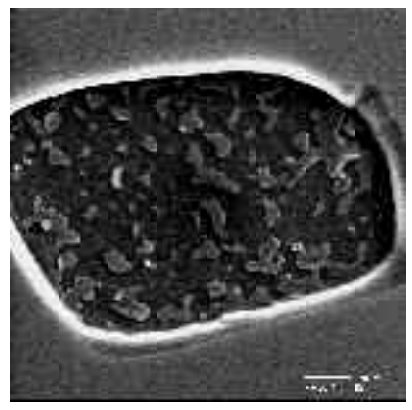

c)

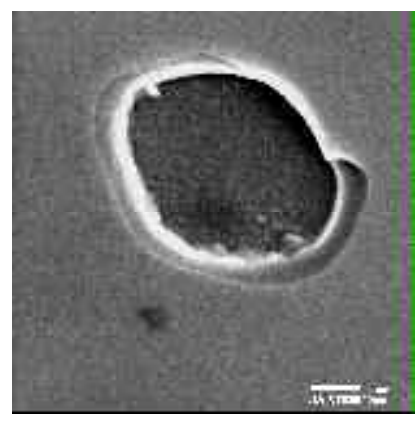

\section{cross-section}
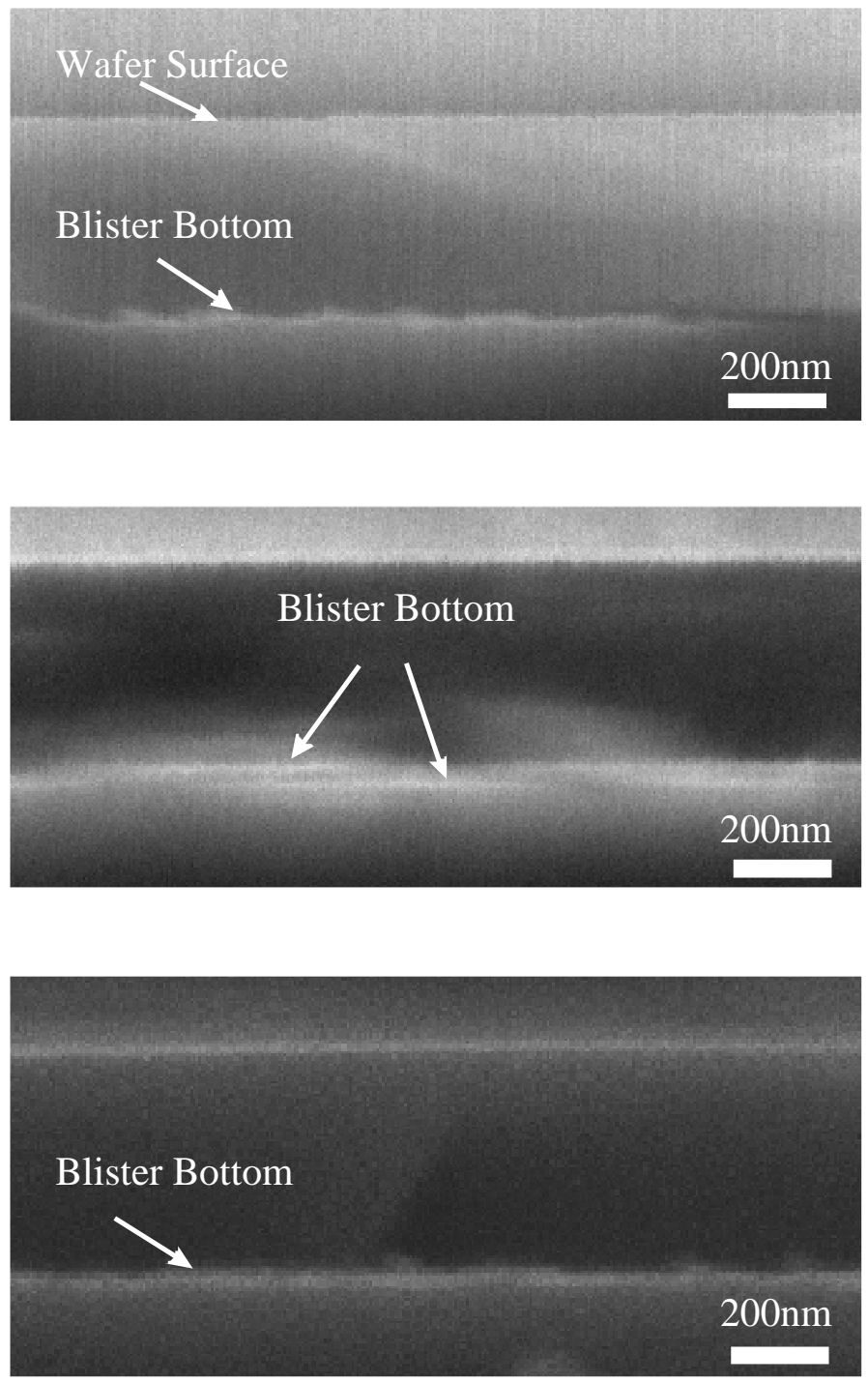

Figure 5-27: SEM plane view and cross-section images of a "popped off" surface blister from the samples implanted to a H-dose of $5 \times 10^{16} \mathrm{~cm}^{-2}(\mathrm{a}), 7 \times 10^{16} \mathrm{~cm}^{-2}(\mathrm{~b})$, and $1 \times 10^{17} \mathrm{~cm}^{-2}$ (c).

Cross-section SEM images were also obtained from sample areas, where no surface blistering occurred. Figure 5-28 shows these images. The bright lines indicate micro cracks, which did not lead to the delamination of the silicon over layer. Measurements of the depth of these micro cracks reveal, that their depth location is equal to the depth of the surface blisters, verifying the assumption, that coalescence of the micro cracks leads to ion-cutting. 


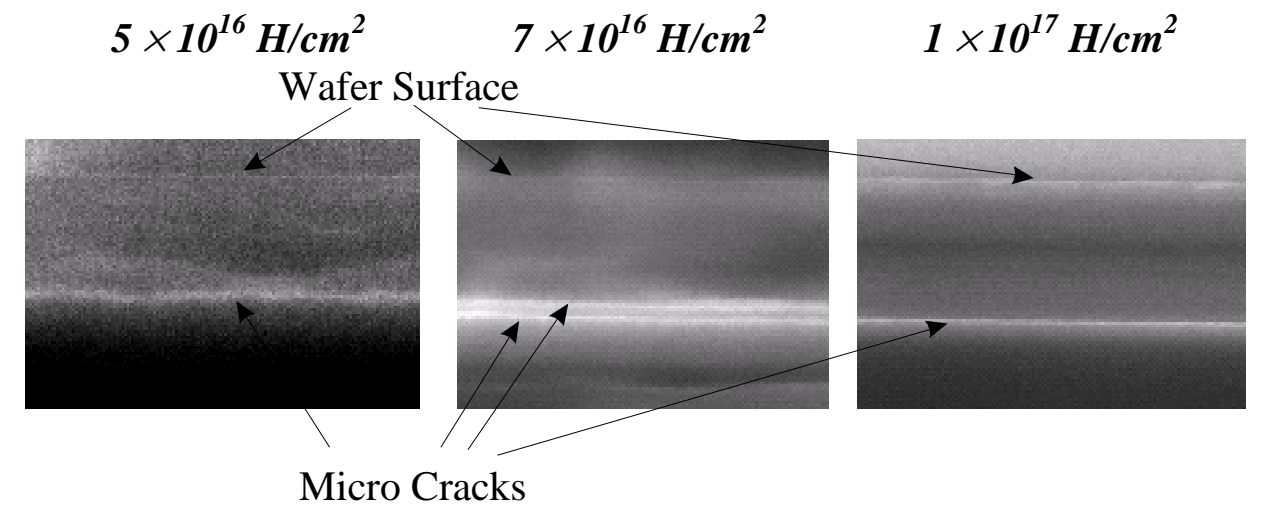

Figure 5-28: SEM cross-section images of non-blistered areas from the samples implanted to a different $\mathrm{H}$-doses and annealed at $600^{\circ} \mathrm{C}$ for 30 minutes.

To confirm the XSEM results and to get quantitative information about the blister bottom roughness as a function of hydrogen ion dose, AFM measurements were performed. Figure 5-29 shows the AFM traces for samples exposed to $5 \times 10^{16} \mathrm{H} / \mathrm{cm}^{2}$, $7 \times 10^{16} \mathrm{H} / \mathrm{cm}^{2}$, and $1 \times 10^{17} \mathrm{H} / \mathrm{cm}^{2}$. These data show that blisters obtained from samples implanted with the extreme doses of $5 \times 10^{16} \mathrm{H} / \mathrm{cm}^{2}$ and $1 \times 10^{17} \mathrm{H} / \mathrm{cm}^{2}$ have nearly flat bottoms and that the blister depth jumps from the shallow cut location at the low hydrogen dose to the deep cut location at the high hydrogen dose. The blister bottom morphology resulting from the intermediate implantation dose of $7 \times 10^{16} \mathrm{H} / \mathrm{cm}^{2}$ shows the blister depth to alternate between the shallow and the deep cut location.

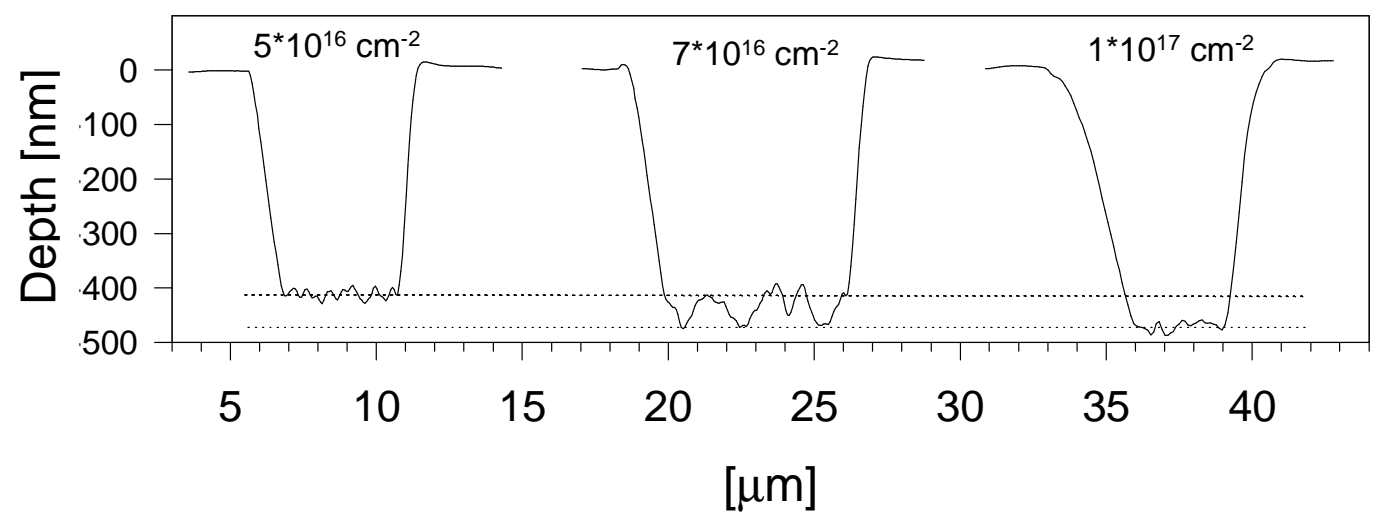

Figure 5-29: AFM cross-section images of a surface blister of each sample after $\mathrm{H}$-implantations to different doses and annealing at $600{ }^{\circ} \mathrm{C}$ for 30 minutes.

Averaging over the entire blister bottom area, the AFM measurements reveal that the shallow cut location is $418 \mathrm{~nm}$ deep and the deep cut location at a depth of $457 \mathrm{~nm}$. The average depths of the upper and lower level of the blister bottom for the sample implanted with the intermediate dose were measured to be the same observed for the 
$5 \times 10^{16} \mathrm{H} / \mathrm{cm}^{2}$ and $1 \times 10^{17} \mathrm{H} / \mathrm{cm}^{2}$ implantations, respectively. Thus, the AFM measurements confirm the blister morphology and cleavage depths obtained by XSEM.
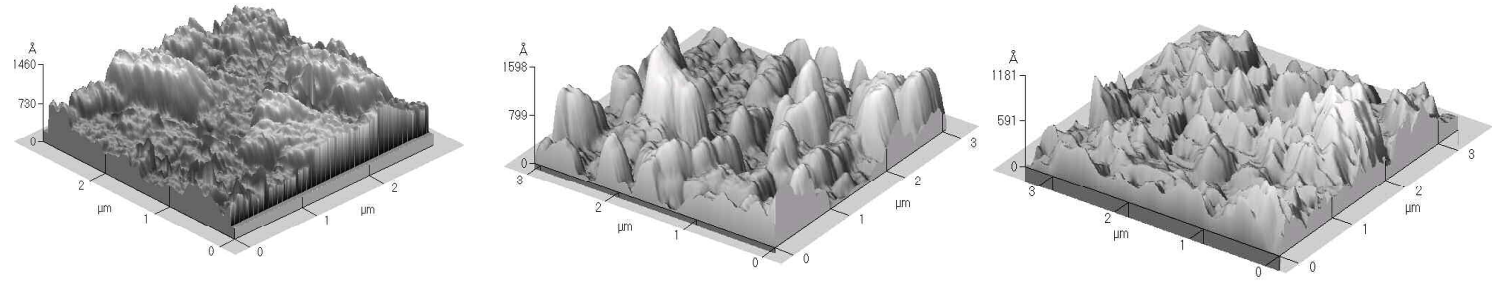

Figure 5-30: AFM 3-dimensional images of a surface blister bottom of each sample after H-implantations to different doses and annealing at $600{ }^{\circ} \mathrm{C}$ for 30 minutes. Note that the y-axes have different scales.

Figure 5-30 shows three-dimensional images of a blister bottom for the samples implanted to $5 \times 10^{16} \mathrm{H} / \mathrm{cm}^{2}, 7 \times 10^{16} \mathrm{H} / \mathrm{cm}^{2}$, and $1 \times 10^{17} \mathrm{H} / \mathrm{cm}^{2}$. The blister bottom of the sample exposed to $7 \times 10^{16} \mathrm{H} / \mathrm{cm}^{2}$ shows clearly the oscillation of the Ion-Cut between the two depths. The rms values of the blister bottom roughness (see equation 4-24) was measured to be $25 \mathrm{~nm}$ for the $5 \times 10^{16} \mathrm{H} / \mathrm{cm}^{2}$ implanted sample and $19 \mathrm{~nm}$ for the sample irradiated to a dose of $1 \times 10^{17} \mathrm{H} / \mathrm{cm}^{2}$. Thus, the AFM measurements of the Ion-Cut roughness verify the optical appearance of the surface blisters in the SEM plane view images (see figure 5-27).

Microstructural information was obtained by XTEM, showing the cleavage morphology from implanted and annealed silicon in non-blistered areas of the samples. Figure 5-31 presents as an example a low magnification XTEM image from a silicon wafer implanted with $7 \times 10^{16} \mathrm{H} / \mathrm{cm}^{2}$ after the anneal. The image shows a $150 \mathrm{~nm}$ broad region of dense damage, induced by the $\mathrm{H}$-ion irradiation. Clearly visible are micro cracks that permeate the implantation zone at two distinct depths. Above the implantation zone, the wafer appears to contain low damage. This is verified by the corresponding electron diffraction pattern (not shown), which indicates good crystalline quality in this region. Since the anneal temperature is high and the anneal duration long, the Ostwald ripening process is very advanced. Large H-platelets grew on the expense of smaller ones, and coalesced into micro cracks. Therefore, no H-platelets like the ones observed in figure 5-9 are visible. Interestingly, the image reveals, that micro cracks never form at both depths, 
simultaneously. This indicates a competing behavior of these two depth locations in the Ostwald ripening process. The H-atoms either diffuse to the center or the end of tail region of the damage zone.

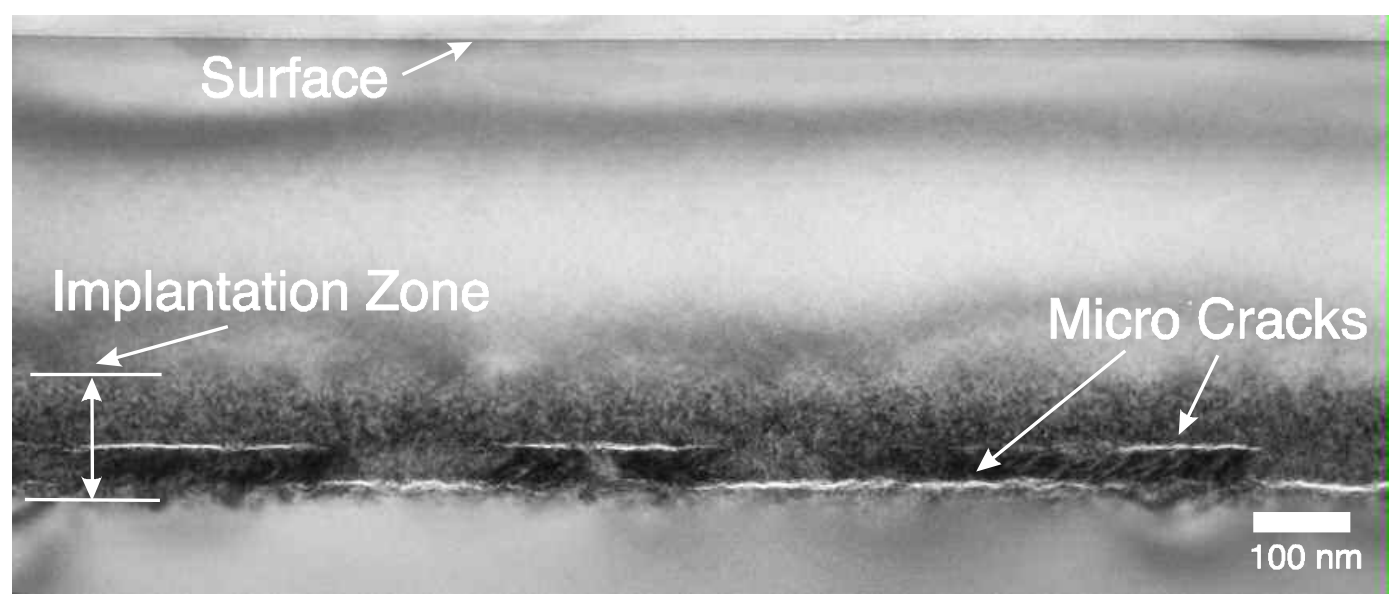

Figure 5-31: Low magnification cross-sectional TEM bright-field images from a single crystal $<100\rangle$ silicon wafer, $\mathrm{H}$ ion-irradiated under cryogenic conditions with $42 \mathrm{keV} \mathrm{H}$-ions to a dose of $7 \times 10^{16} \mathrm{H} / \mathrm{cm}^{2}$ and subsequently annealed at $600{ }^{\circ} \mathrm{C}$ for 30 minutes, viewed edge on in the [110] silicon projection. The image shows the buried densely damaged implantation zone, permeated with micro cracks at two distinct depths.

Figure 5-32 shows higher magnification XTEM images from silicon implanted with hydrogen to doses of $5 \times 10^{16} \mathrm{~cm}^{-2}$ (a), $7 \times 10^{16} \mathrm{~cm}^{-2}$ (b), and $1 \times 10^{17} \mathrm{~cm}^{-2}$ (c). These images show that the micro cracks appear either in the center or at the end of the damaged region. The XTEM determined average depth locations of the micro cracks was measured to be $418 \mathrm{~nm}$ for the sample implanted with $5 \times 10^{16} \mathrm{H} / \mathrm{cm}^{2}$, and $457 \mathrm{~nm}$ for the sample, implanted to a dose of $1 \times 10^{17} \mathrm{H} / \mathrm{cm}^{2}$, in very good agreement with the XSEM measurements (see figure 5-28). The two distinct depths where micro cracks are forming in the sample implanted with $7 \times 10^{16} \mathrm{H} / \mathrm{cm}^{2}$, have average depths of $418 \mathrm{~nm}$ and $456 \mathrm{~nm}$, equal to the blister depth in the sample with a H-implantation dose of $5 \times 10^{16} \mathrm{~cm}^{-2}$ and $1 \times 10^{17} \mathrm{~cm}^{-2}$, respectively. These results are in excellent agreement with the XSEM observations that reveal that there are two distinct depths where micro cracks occur and that these depths are dependent on the hydrogen implantation dose.

The XTEM images are also in accordance with blister bottom roughness measurements by AFM. The micro cracks permeating the sample with a H-implantation dose of $5 \times 10^{16} \mathrm{~cm}^{-2}$ vary more in depth than the ones in the sample implanted to $1 \times 10^{17} \mathrm{H} / \mathrm{cm}^{2}$. 

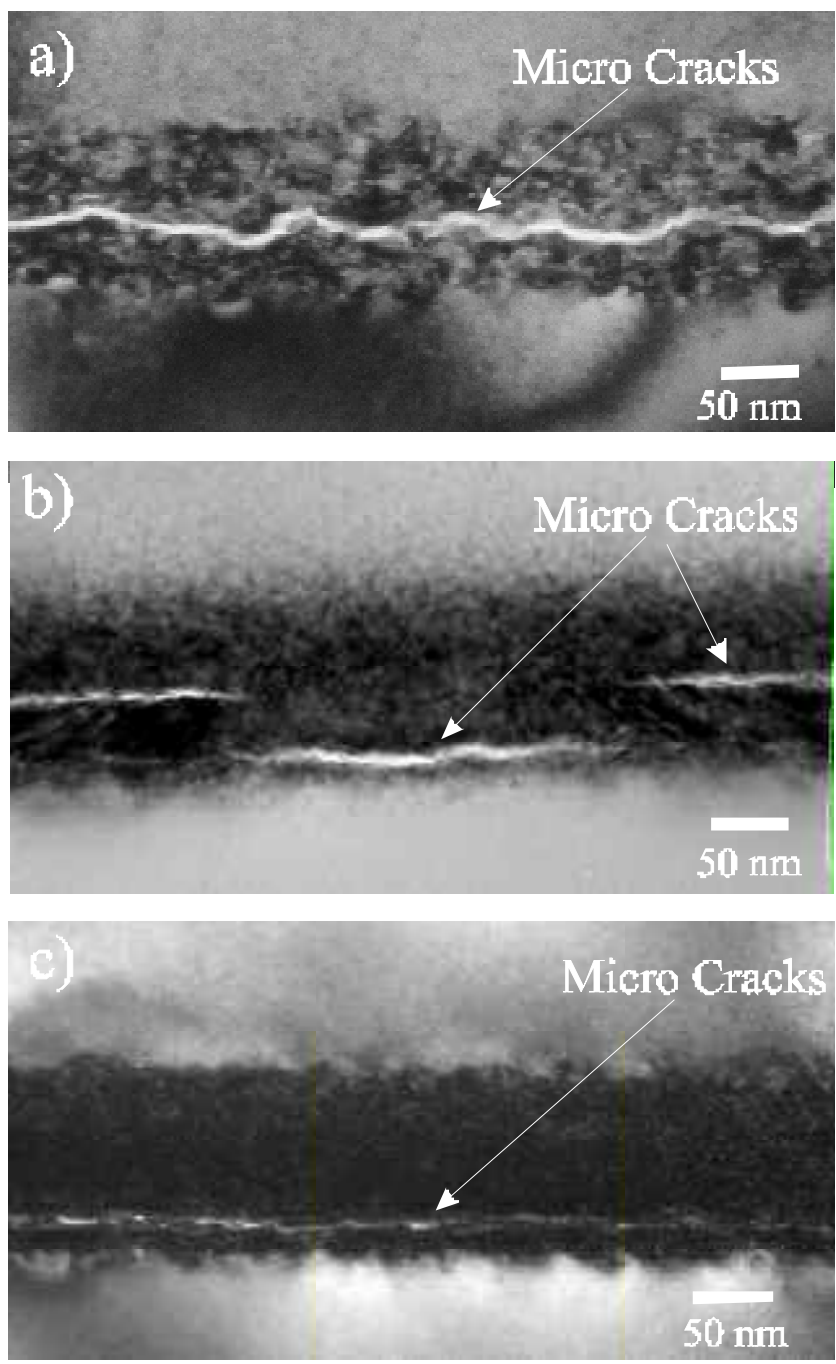

Figure 5-32:

High magnification cross-sectional TEM bright-field images from single crystal $\langle 100\rangle$ silicon wafers, viewed edge on in the [110] silicon projection. The wafers were $\mathrm{H}$-ion-implanted under cryogenic conditions with 42 $\mathrm{keV} \mathrm{H}$-ions to a dose of (a) $5 \times 10^{16} \mathrm{H} / \mathrm{cm}^{2}, \quad$ (b) $7 \times 10^{16} \mathrm{H} / \mathrm{cm}^{2}$, and (c) $1 \times 10^{17} \mathrm{H} / \mathrm{cm}^{2}, \quad$ and subsequently annealed at $600{ }^{\circ} \mathrm{C}$ for 30 minutes. The images show micro crack propagation throughout the implantation zone at different depths, dependent on the implantation dose. (d) The electron diffraction pattern from the implantation zone in the sample implanted to a dose $1 \times 10^{17} \mathrm{H} / \mathrm{cm}^{2}$.

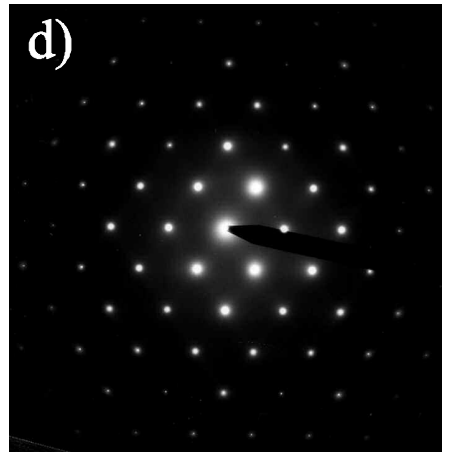

The electron diffraction pattern, taken from the implantation zone in the sample with the highest dose (figure 5-32 (d)) reveals that no amorphous regions formed during the $\mathrm{H}$ implantation process. Reexamining the RBS channeling data, shown in figure 5-22, the electron diffraction pattern confirms the RBS channeling analysis. Although, the direct backscattering yield for the $1 \times 10^{17} \mathrm{H} / \mathrm{cm}^{2}$ implant reaches the yield of the random signal, no amorphization took place. The direct backscattering yield had to be subtracted from the baseline; in other words, the yield in the RBS channeling spectrum is the sum of direct backscattering and dechanneling of the analyzing beam and did not evolve due to direct backscattering events only.

XTEM images, obtained from different region in the sample, implanted to the highest $\mathrm{H}$-dose and annealed, show $\mathrm{H}_{2}$-gas bubbles instead of micro cracks in the end of tail region of the implantation zone (see figure 5-33). This observation verifies the 
proposed evolution of the $\mathrm{H}$-implant into micro cracks. The $\mathrm{H}$-atoms accumulated into $\mathrm{H}_{2}$-gas bubbles, but their coalescence, which would lead to the formation of micro cracks, did not take place, yet.

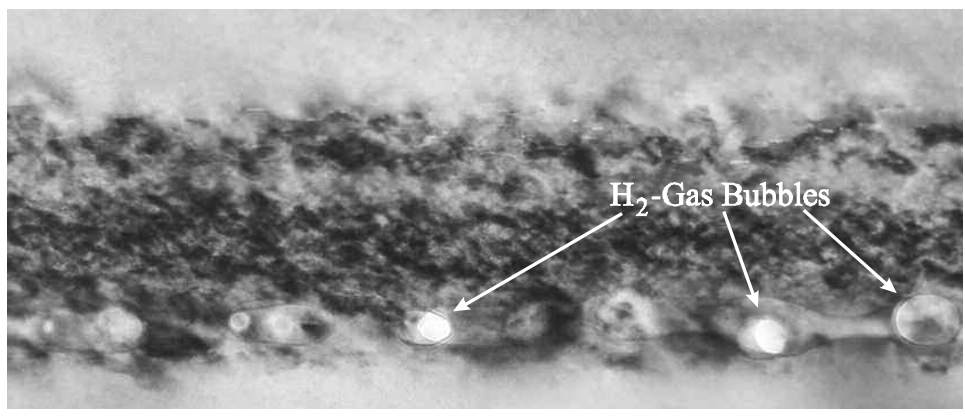

Figure 5-33: High magnification cross-sectional TEM bright-field images from a single crystal $<100>$ silicon wafer, viewed edge on in the [110] silicon projection. The wafer was implanted to a dose of $1 \times 10^{17} \mathrm{H} / \mathrm{cm}^{2}$, and subsequently annealed at $600{ }^{\circ} \mathrm{C}$ for $30 \mathrm{~min}$. The image shows $\mathrm{H}_{2-}$ gas bubbles forming at the end of tail region in the implantation damage zone.

XTEM images could also be acquired from sample regions, where surface blistering occurred. Figure 5-34 shows such areas for the samples, implanted to $5 \times 10^{16} \mathrm{H} / \mathrm{cm}^{2}$ and $1 \times 10^{17} \mathrm{H} / \mathrm{cm}^{2}$. The images confirm the microcracks to be the location where the actual Ion-Cut occurs. Figure 5-34 b shows the blister bottom and the popped off silicon overlayer, indicating that the exfoliation took place during the TEM sample preparation. The TEM samples undergoes a minor anneal to harden the epoxy glue (see chapter 4.4). During this anneal the silicon over layer exfoliated and was held in place by the hardening glue. Comparing the thickness of the damage zone in the donor wafer and the silicon over layer, the measurements reveal, that the Ion-Cut depth locations and the micro cracks depth locations (see figure 5-32) are the same.

a)

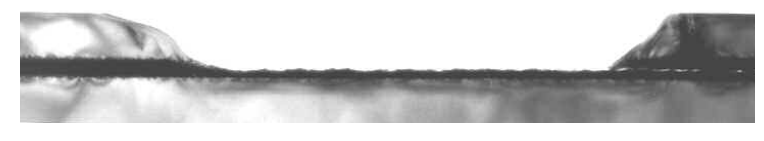

b)

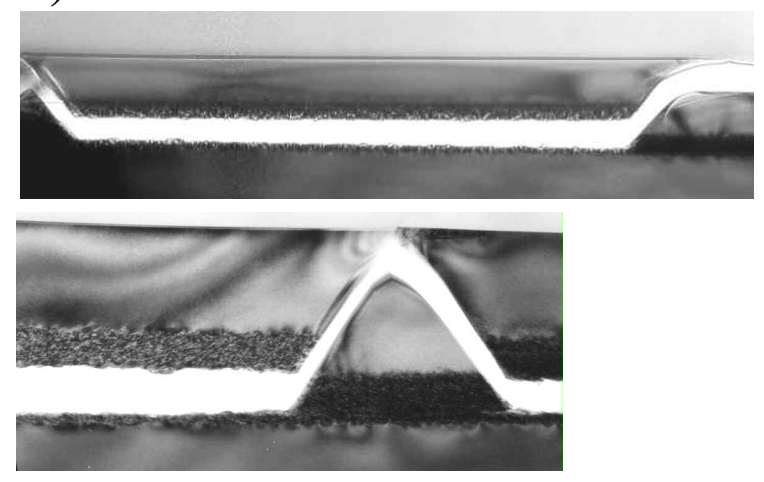

Figure 5-34: XTEM bright-field images from surface blistered areas, viewed edge on in the [110] silicon projection. Low magnification image from the sample implanted to $5 \times 10^{16} \mathrm{H} / \mathrm{cm}^{2}$ (a); low and higher magnification images from the sample implanted to $1 \times 10^{17} \mathrm{H} / \mathrm{cm}^{2}$ (b). 
Summarizing the results of the ion beam analysis and the Ion-Cut depth measurements, figure 5-35 shows the hydrogen concentration depth distribution, the damage depth profile and the Ion-Cut depth for each sample, which developed surface blistering.
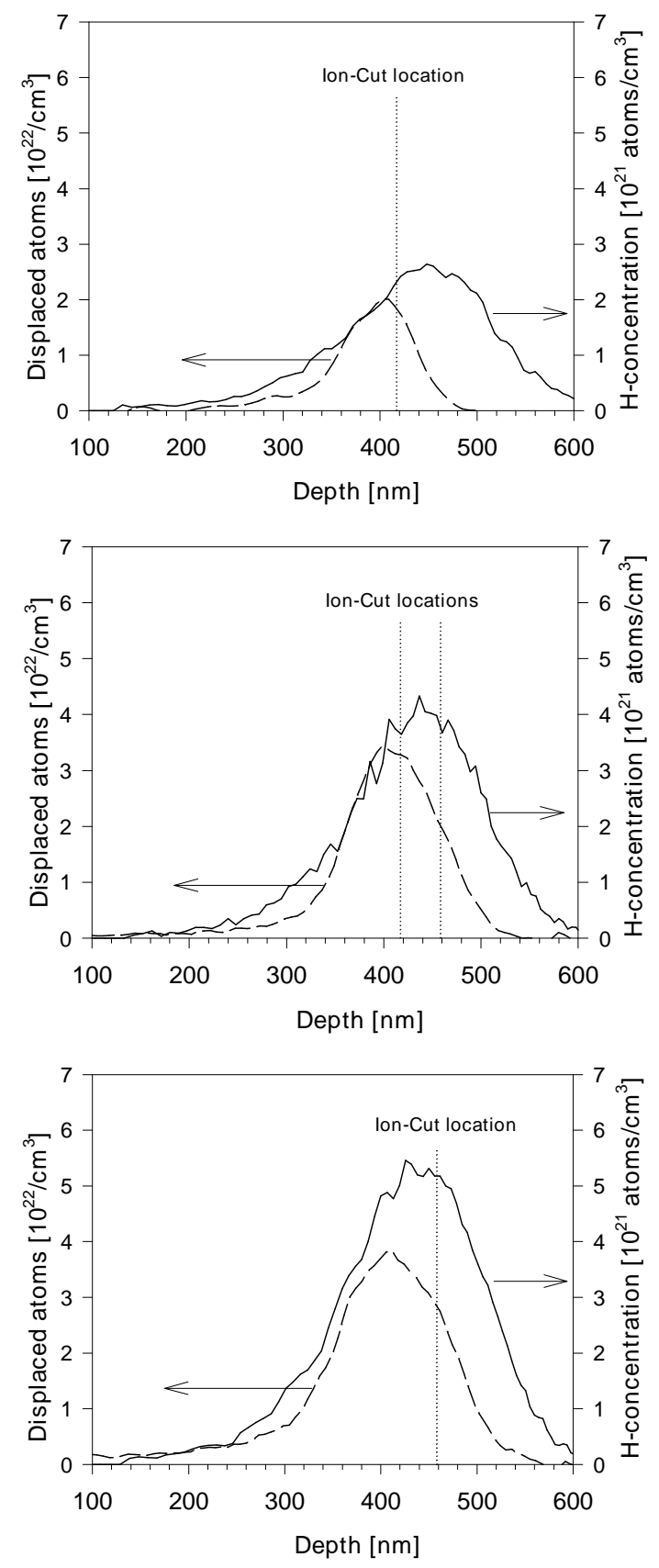

\section{$5 \times 10^{16} \mathrm{H} / \mathrm{cm}^{2}$}

$7 \times 10^{16} \mathrm{H} / \mathrm{cm}^{2}$

\section{$1 \times 10^{17} \mathbf{H} / \mathrm{cm}^{2}$}

Figure 5-35: H-concentration depth profiles, implantation damage depth distributions, and Ion-Cut depth locations for the samples implanted to doses of $5 \times 10^{16} \mathrm{H} / \mathrm{cm}^{2}, 7 \times 10^{16} \mathrm{H} / \mathrm{cm}^{2}$, and $1 \times 10^{17} \mathrm{H} / \mathrm{cm}^{2}$.

At an implantation dose $5 \times 10^{16} \mathrm{~cm}^{-2}$ cleavage occurs short of the peak in the hydrogen concentration and is collocated with the maximum in the damage distribution. At $1 \times 10^{17} \mathrm{~cm}^{-2}$ the cleavage occurs at a depth slightly larger than the hydrogen concentration peak, and at $7 \times 10^{16} \mathrm{~cm}^{-2}$ cleavage occurs at both locations. 


\subsubsection{Influence of the Silicon Fracture Toughness on the Ion-Cut}

A comparison of the depth locations of the damage peak, the maximum hydrogen concentration, and the two distinct Ion-Cut locations (see figure 5-35) show that cleavage of the silicon occurs either at the depth of maximum damage (implantation dose: 5 and $7 \times 10^{16} \mathrm{~cm}^{-2}$ ) or at the deeper part of the H-depth-distribution (implantation dose: 7 and $10 \times 10^{16} \mathrm{~cm}^{-2}$ ), but never at the depth of the hydrogen concentration peak. To understand why cleavage occurs at these two depth locations requires an examination of the mechanisms behind the $\mathrm{H}_{2}$-gas bubble nucleation and growth.

The shallow location of the ion-cut was found to take place in the depth region of high damage rather than at the highest $\mathrm{H}$-concentration. As described in detail in chapter 5.1.5, this phenomenon was attributed to the compressive biaxial stress distribution in the implantation zone and the corresponding large tensile out-of-plane strain field and its influence on the (100) H-platelet formation.

To strengthen the assumption that the damage and the elastic out-of-plane strain depth distributions coincide it is necessary to clarify that the H-implant itself, whose concentration depth profile is centered somewhat deeper in the material, does not contribute to the strain build up in the material. Earlier research has been shown that hydrogen itself does not produce any appreciable displacement field in silicon. $[42,136]$ In addition, the $\mathrm{H}$-induced strain build up is attributed to two particular $\mathrm{H}$-configurations, i.e. the so-called $\mathrm{H}^{*}$ and $\mathrm{H}^{* * *}$ complexes. [136] However, when the H-implantation is carried out at liquid nitrogen temperature like in this study, the $\mathrm{H}^{*}$ complex, an interstitial $\mathrm{H}_{2}$ molecule, is only generated upon annealing at $\mathrm{T} \geq 150{ }^{\circ} \mathrm{C}$. The $\mathrm{H}^{* *}$ configuration, an accumulation of several $\mathrm{H}_{2}$ molecules located in small vacancy clusters, arises only during annealing at $\mathrm{T} \geq 300{ }^{\circ} \mathrm{C}$. [136] Therefore, in the as-implanted sample studied here no $\mathrm{H}^{*}$ and $\mathrm{H}^{* * *}$ configurations are expected to be present and thus the strain contribution by the hydrogen itself is expected to be negligible. Consequently, I assume the elastic out-of-plane tensile strain to be generated by the implantation damage only and to scale with the defect concentration, plotted in figure 5-23 (p. 95).

Unlike for the sample exposed to $5 \times 10^{16} \mathrm{H} / \mathrm{cm}^{2}$, the Ion-Cut location shifts to a larger depth, when implanted with $1 \times 10^{17} \mathrm{H} / \mathrm{cm}^{2}$ and at an intermediate dose of $7 \times 10^{16} \mathrm{H} / \mathrm{cm}^{2}$ ion-cutting occurs at both depths.

The explanation of these observations requires further examination of the evolution of the biaxial compressive stress field and the corresponding out-of-plain tensile strain field with increasing H-implantation dose. 
Therefore, the integrated compressive stress field in the silicon samples after the $\mathrm{H}$-implantations was monitored by measuring the sample curvature changes, developed during the implantation process as described in chapter 4.5. The measurements were performed before the heat treatment of the implanted samples. Figure 5-36 shows the results of the wafer curvature measurements.

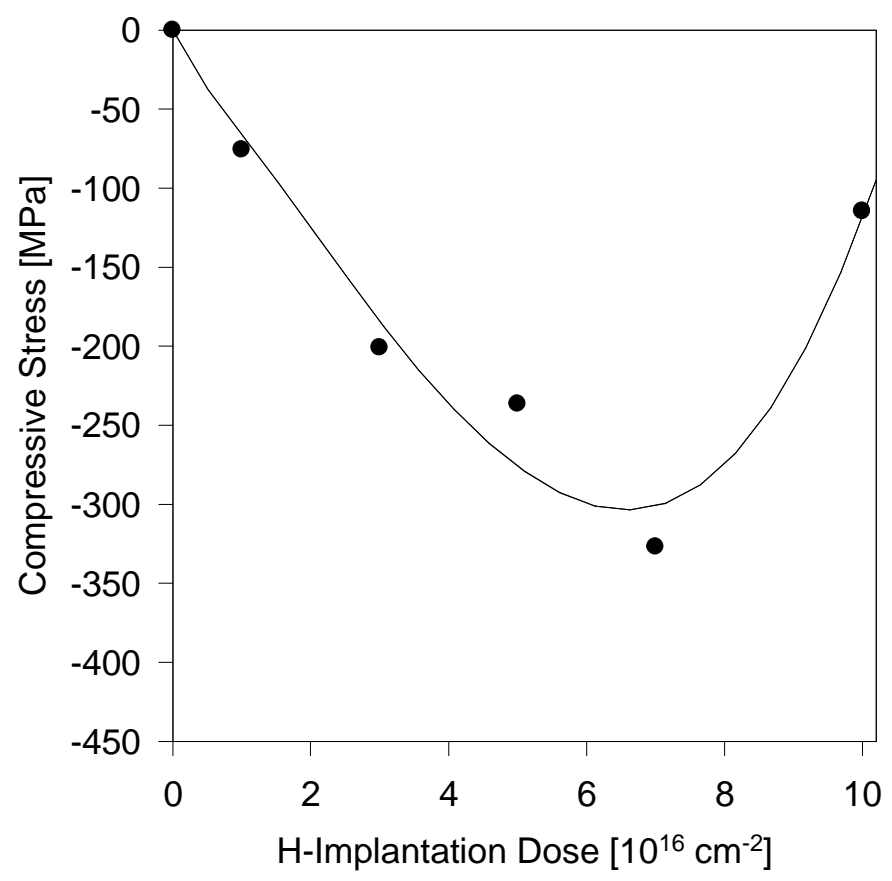

Figure 5-36: Integrated compressive stress in the implantation zone, calculated by the curvature change of the silicon wafers during the $\mathrm{H}$-ion implantations (see equation (4-26)). The line serves only as a guide to the eye.

The measurements were performed for all five different $\mathrm{H}$-implantation doses. As the graph shows, the compressive stress in the implantation zone increases with increasing $\mathrm{H}$-implantation dose up to $7 \times 10^{16} \mathrm{H} / \mathrm{cm}^{2}$. When implanted to even higher doses, i.e. $1 \times 10^{17} \mathrm{H} / \mathrm{cm}^{2}$, the integrated compressive stress in the implantation zone decreases rapidly.

To relate the stress measurements to the sample morphology, it is necessary to reexamine the RBS channeling spectra in figure 5-22 (p. 94). The RBS channeling spectra show that with increasing hydrogen dose the accumulation of radiation damage also increases. Previous research has shown that at low ion-implantation doses, the inplane compressive stress and the corresponding elastic out-of plane tensile strain scales linearly with the ion-implantation dose. [135] This is in accordance with the strain evolutions observed here for implantation doses ranging from 1 to $7 \times 10^{16} \mathrm{H} / \mathrm{cm}^{2}$. 
As established above, H-complexes are not the source of the displacement field in the implantation zone. Therefore, intrinsic lattice defects are responsible for the build up of the compressive in-plane stress in the implantation zone. Earlier research has shown, that the relaxation volumes of single vacancies and Si-self-interstitials have the same absolute value and opposite signs and therefore cancel each other. $[137,138]$ Consequently, Frenkel pairs do not provoke any volume change, which would generate compressive stresses. However, single vacancies in isolated Si self-interstitials are very mobile at room temperature. $[139,140]$ Their high mobility in combination with the high amount of displaced $\mathrm{Si}$ atoms in the implantation damage zone results in an intrinsic defect evolution, which is entirely governed by the recombination of Frenkel pairs and the aggregation of vacancies and self-interstitials inside the damaged region. [140,141] Therefore, I assume that vacancy and Si-self-interstitial clusters are the intrinsic defect species responsible for the build up of the measured in-plane compressive stress.

When implanted to even higher doses, i.e. $1 \times 10^{17} \mathrm{H} / \mathrm{cm}^{2}$, the integrated compressive stress in the implantation zone decreases rapidly (see figure 5-36). Previous research has shown, that plastic flow occurs in amorphous silicon [128,142,143], which leads to a relaxation of the silicon lattice and consequently to a rapid decrease in the biaxial in-plane stress. For the sample implanted to the highest H-dose, the direct backscattering yield reaches the yield of the randomly aligned spectrum. However, XTEM electron diffraction patterns (not shown) revealed that, although a heavily damaged crystalline region had been formed, the $\mathrm{H}$-ion implantation process had not created any amorphous clusters. Therefore, the observed compressive stress relief took place before the ion-irradiation would have caused amorphization and hence plastic flow in amorphous silicon is not the mechanism by which the stress relief was achieved.

In the present case, the rapid stress decrease can be explained by the migration and rearrangement of lattice defects during the ion irradiation process. The presence of a damage distribution indicates that the stress is not uniform but is also distributed. Nonuniform stress leads to a gradient in the chemical potential and thus provides the driving force for the migration of defects such that stress relief is accomplished. [132] Previous research has suggested that ion-irradiation considerably enhances the stress relieving diffusion of defects in crystalline silicon. [128] Therefore, I attribute the rapid decrease of the compressive stress in the sample implanted to $1 \times 10^{17} \mathrm{H} / \mathrm{cm}^{2}$ to a defect migration and rearrangement in the implantation damage zone during the irradiation process itself: As mentioned above, the compressive stress was attributed mainly to vacancy and $\mathrm{Si}$ self-interstitial clusters. Earlier research has shown that defect clusters in metals align in the presence of tensile stress normal to the tensile strain axis. [144] I hypothesize that 
similar phenomena occur here. The compressive in-plane stress causes the Si selfinterstitial clusters to preferentially align normal to the tensile strain axis and the vacancy clusters to preferentially align parallel to the tensile axis. This defect migration results in the formation of inelastic out-of-plane strain and the reduction of the in-plane biaxial compressive stress. The decrease of the in-plane compressive stress in the heavily damaged zone consequently leads to a decrease of the (100) H-platelet formation enhancing elastic out-of-plane strain. This reduces the preference of the region of maximum damage for (100) H-platelet formation.

However, at the periphery of the damage distribution, where the damage density and thus the compressive biaxial in-plane stress are not large enough to induce stress relief by defect rearrangement, the (100) H-platelet formation enhancing elastic out-ofplane tensile strain is maintained, as confirmed experimentally on ion-implanted semiconductor material. [145]

Therefore, as the $\mathrm{H}$ dose increases, the optimum location for cleavage will shift away from the damage peak, and move towards the periphery of the implantation zone where the damage is lower, the tensile out-of-plane strain sufficient, and the hydrogen concentration is high enough to promote (100) H-platelet formation. Examining the damage and hydrogen distribution shown in figure 5-35 (p. 105), it is clear that a large amount of hydrogen is only present at a deeper, rather than a shallower, location.

The transition of the Ion-Cut to larger depths is not caused only by the decrease of the out-of-plane tensile strain in the region of maximum damage: The sample, implanted to the intermediate dose of $7 \times 10^{16} \mathrm{H} / \mathrm{cm}^{2}$ shows only partly cleavage near the damage peak and also produced micro-cracking in the end of tail region of the implantation zone. However, this sample contains the largest in-plane compressive stress (see figure 5-36) and thus the largest out-of-plane tensile strain. Therefore, the evolution of the biaxial inpane stresses with increasing $\mathrm{H}$-implantation dose alone cannot explain the observed results.

The change in the fracture toughness of silicon with increasing H-implantation dose also has to be considered to understand the shift of the Ion-Cut location towards a larger depth. Research has shown that the fracture toughness of brittle materials initially decreases and then gradually increases with increasing radiation damage. [146] In addition, recent Molecular Dynamics calculations revealed, that the fracture toughness of silicon increases in the presence of high ion-irradiation induced crystal damage. [147] 
Therefore, as $\mathrm{H}$ is implanted into silicon, the fracture toughness in the damage peak region will be locally reduced, thereby facilitating fracture at that location. As the implantation dose is increased $\left(>5 \times 10^{16} \mathrm{H} / \mathrm{cm}^{2}\right.$ in these experiments), several phenomena occur that cause the fracture toughness at the damage peak location to increase. First, with increasing hydrogen dose the accumulation of radiation damage at the damage peak also increases, which in turn toughens silicon to fracture [146,147] while simultaneously increasing the hydrogen concentration in the tails of the distribution.

As the $\mathrm{H}$ dose increases, the number and extent of $\mathrm{H}$-platelets and $\mathrm{H}_{2}$-gas bubbles in the region of maximum damage and maximum $\mathrm{H}$-concentration also increases. This increase in platelet and bubble concentration toughens the region even more by shielding any crack nuclei that would have been operable at lower implantation doses. The understanding of this phenomenon requires a brief examination of the criteria at which a crack at the tip of an $\mathrm{H}_{2}$-gas bubble propagates through the material. Figure 5-37 illustrates the stress field in the material surrounding an $\mathrm{H}_{2}$-gas bubble.

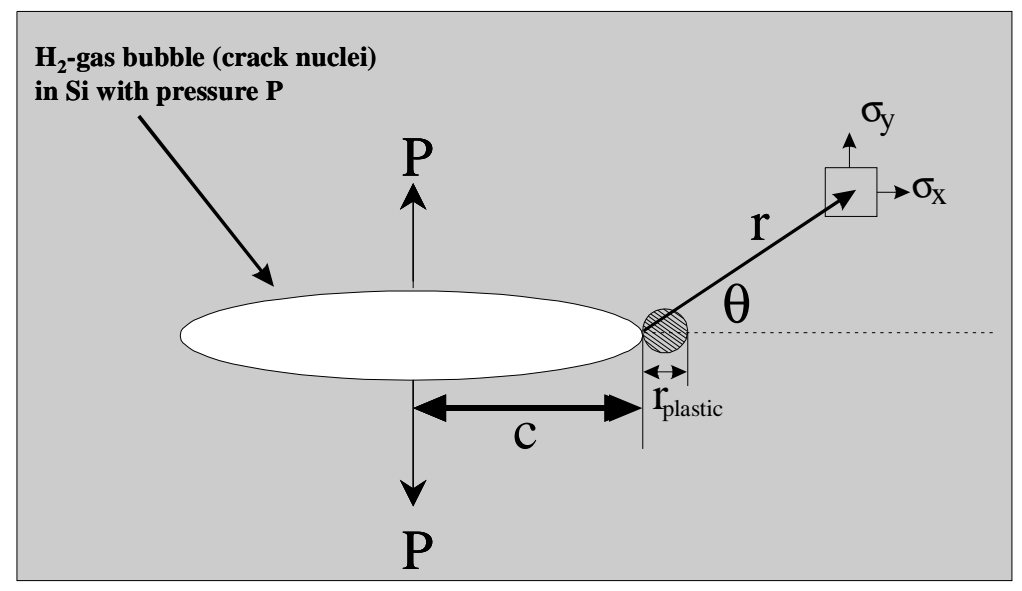

Figure 5-37: Schematic illustration of the crack propagation at the tip of an $\mathrm{H}_{2}$-gas bubble in silicon.

In the case of the $\mathrm{H}_{2}$-gas bubble, the normal stresses give rise to the so-called "opening mode" or mode 1 loading: The displacements of the crack surfaces are perpendicular to the plane of the crack. The elastic stress field in mode 1 can be evaluated as followed: In the coordinate system of figure 5-37 the stress at a distance $r$ from the crack tip is calculated to be [148]:

$$
\sigma_{i j}=\frac{K}{\sqrt{2 \pi r}} \cdot f_{i j}(\theta)
$$


where $\sigma_{i j}$ are the stresses acting on a material element $d x d y$ at a distance $\mathrm{r}$ from the crack tip and at an angle $\theta$ from the crack plane, and $f_{i j}(\theta)$ are known functions of $\theta$. The parameter $K$ is called the stress intensity factor [148]:

$$
K=\frac{2 P}{(c \pi)^{3 / 2}}
$$

where $c$ is half the length of the bubbles. Equation (5-1) is an elastic solution, which does not prohibit the stresses to become infinite at the crack tip. In reality this cannot occur, since plastic deformation takes place at the crack tip, which keeps the crack tip radius $>0$ and thus the stresses at the crack tip finite. An estimate of the size of the crack tip plastic deformation zone can be obtained by determining to which distance $r_{\text {plastic }}$ from the crack tip the elastic stress $\sigma_{y}$ is larger than the yield stress $\sigma_{\text {yield }}$. Fracture, i.e. crack tip propagation through the material, takes place when the elastic stress $\sigma_{y}$ reaches the value of the yield stress $\sigma_{\text {yield }}$ of silicon. Figure 5-38 illustrates the situation, where stress field in the silicon is not due to one H-bubble, but is composed of the sum of stresses induced by a multitude of H-bubbles. The total stress at the crack tip of an H-bubble is reduced by the stress field from surrounding bubbles. Thus, the stress $\sigma_{y}$ at the crack tip decreases and does not reach the value of the yield stress. Therefore, the compressive stresses nearby bubbles induce in the lattice surrounding them exert a crack closing force on a bubble that would have served as the nucleus for fracture at a lower dose.
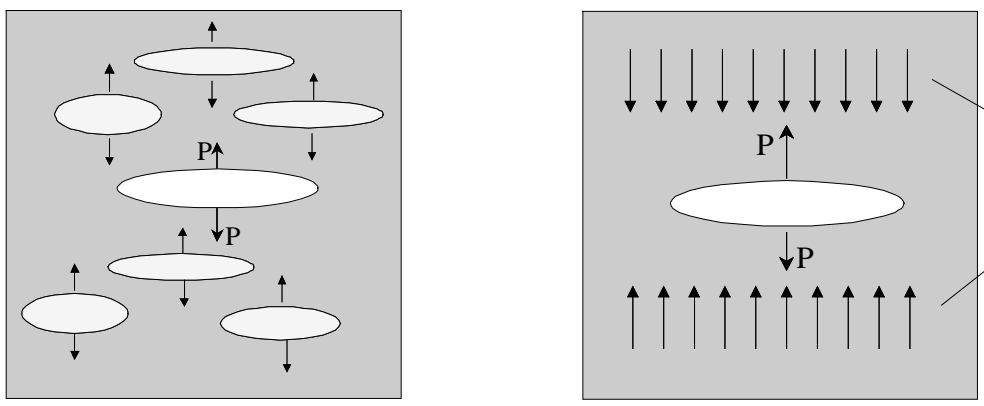

Stress field from surrounding bubbles

Figure 5-38: Schematic illustration of the compressive stress the $\mathrm{H}_{2}$-gas bubbles implement to the nearby silicon lattice and its inhibiting effect on the nucleation and growth on each other.

The overall stress, which is applied to the crack tip, is reduced and hence the fracture toughness increases. This crack tip shielding effect will be maximized for cracks very close to the peak in the damage and hydrogen distribution and will fall off at the extremes of the H-platelet distribution. 
Consequently, the changing fracture mechanics force the Ion-Cut to shift to a region, where the damage is lower and the hydrogen concentration sufficient to promote $\mathrm{H}$-platelet formation on planes parallel to the surface. Examining the damage and hydrogen distribution plotted in Fig. 5-35, it is clear that these criteria can only be met at a deeper, rather than a shallower, location. Consequently, the optimum location for the Ion-Cut moves to a depth beyond the $\mathrm{H}$-concentration peak, where the fracture toughness is low, the implantation damage is high enough for pre-straining the lattice to enhance (100) H-platelet formation, and the hydrogen concentration is high enough for the formation of $\mathrm{H}_{2}$ gas bubbles. At the end of the $\mathrm{H}$-platelet / $\mathrm{H}$-bubble distribution, the $\mathrm{H}$ bubbles shield the crack tips only from one side, making this distinct depth the optimum location of micro crack formation and ion-cutting. The sample with an implantation dose of $7 \times 10^{16} \mathrm{~cm}^{-2}$ represents an intermediate phase where cleavage conditions are equal at both sites.

To prove the hypothesis made in terms of the influence of the changing fracture toughness on the Ion-Cut location, a silicon sample was implanted to a H-dose high enough to cause the formation of an amorphous layer. An implantation dose of $2 \times 10^{17} \mathrm{H} / \mathrm{cm}^{2}$ was chosen. The H-ion implantation was carried out with the same implantation parameters used for the samples studied above. Figure 5-39 (a) plots the ERD spectrum of the as-implanted sample. The spectrum was analyzed as described in chapter 4.1.2. The result of the ERD analysis is shown in figure 5-39 (b). A comparison
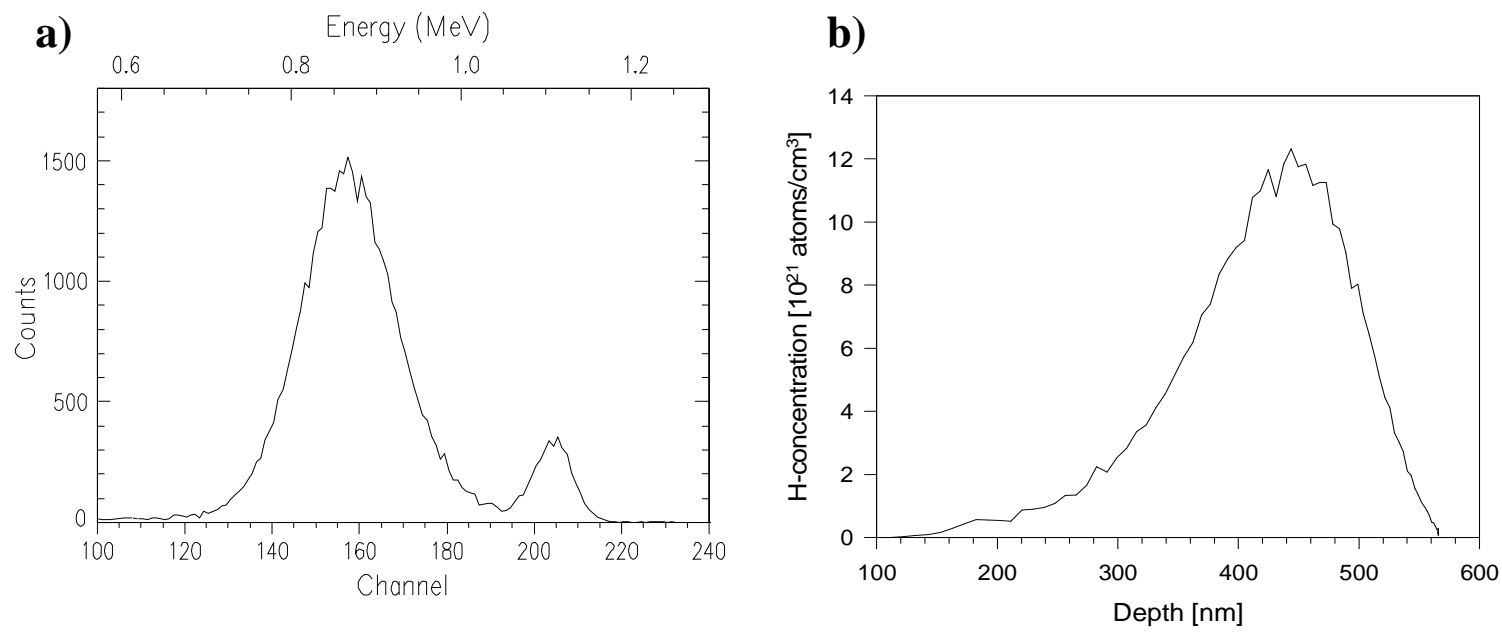

Figure 5-39: (a) $3.0 \mathrm{MeV}$ He ERD spectrum of the $42 \mathrm{keV} \mathrm{H}$-ion implanted $<100>\mathrm{Si}$ in the as-implanted state. The H-implantation dose was $2 \times 10^{17} \mathrm{~cm}^{-2}$. (b) In-depth distribution of the as-implanted hydrogen concentration, deduced from the ERD spectrum. 
of the H-concentration depth distribution with the one from the samples implanted to lower H-doses (figure 5-25) reveals, that the shape of the H-concentration depth profile remains unchanged with increasing $\mathrm{H}$-implantation dose. As in the samples, implanted to lower H-doses, the H-concentration peak was measured to be $445 \mathrm{~nm}$ deep.

Figure 5-40 shows the RBS channeling spectrum of this sample in the asimplanted state. Simulation of the spectrum showed, that the high H-dose implant produces an amorphous silicon layer, extending from a depth of $145 \mathrm{~nm}$ to $465 \mathrm{~nm}$. When the ion irradiation dose is high enough to cause an amorphous silicon crystal layer to form, further ion irradiation leads to the growth of this layer towards the surface. Therefore, the heavily damaged region in the silicon sample extends far more to the surface compared to the silicon sample, implanted to lower H-doses.

Upon annealing at $600{ }^{\circ} \mathrm{C}$ for 30 minutes, intense surface blistering occurs, complicating an accurate RBS channeling analysis of the remaining implantation damage: Unlike in non-blistered surface regions, in areas, where the wafer surface blisters, the blister bottom is exposed and thus becomes the depth, where the analyzing beam starts to penetrate the material. Thus, the implantation damage in blistered areas cause a large backscattering yield at high energies. Consequently, the RBS channeling yield is composed of two overlaying spectra: one resulting from the non-blistered sample surface areas and one resulting from blistered surface areas.
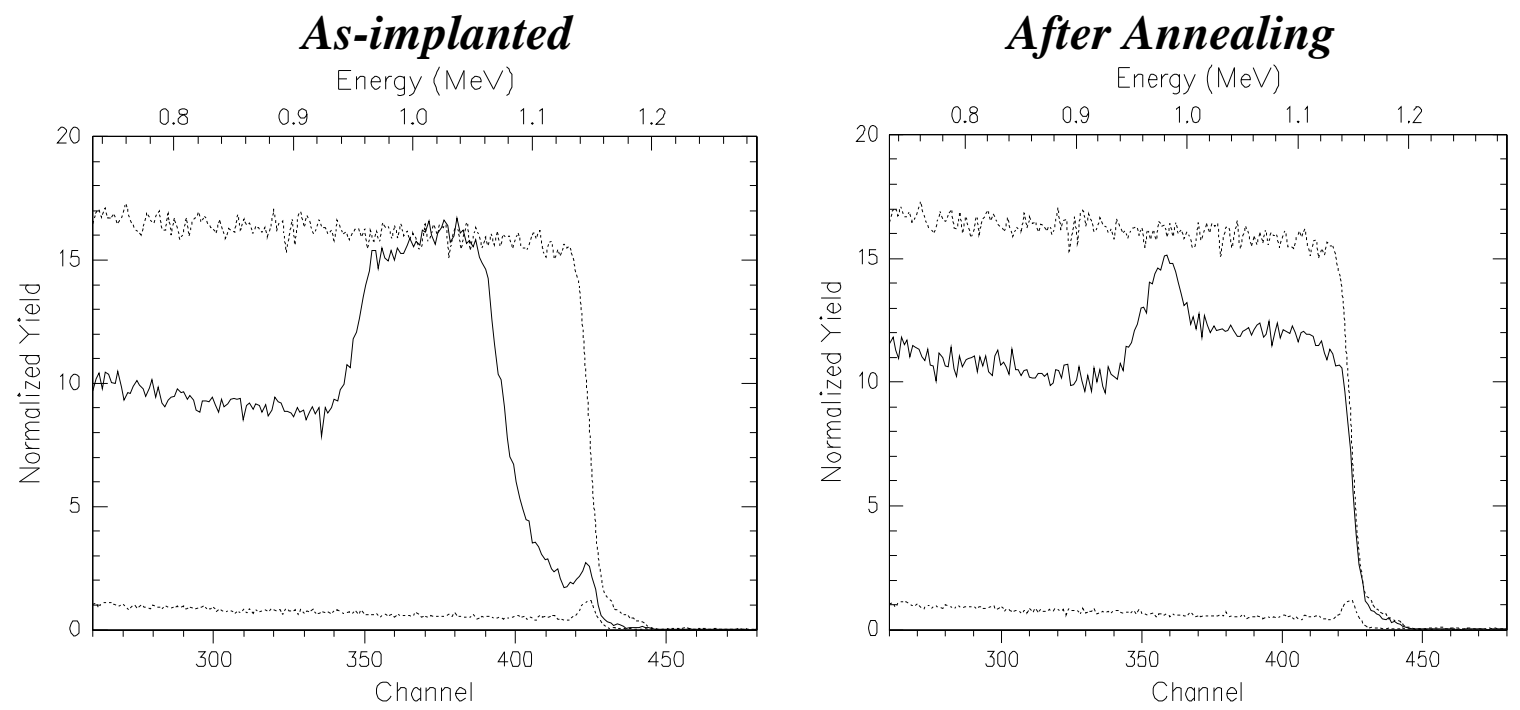

Figure 5-40: $2.0 \mathrm{MeV}$ He RBS-channeling spectrum of the $42 \mathrm{keV} \mathrm{H}$-ion implanted $<100>\mathrm{Si}$ in the asimplanted state (left side) and after annealing at $600{ }^{\circ} \mathrm{C}$ for 30 minutes in vacuum. The $\mathrm{H}$-implantation dose was $2 \times 10^{17} \mathrm{~cm}^{-2}$. Also shown for comparison are the RBS spectra of untreated silicon at random alignment and in channeling condition (dashed lines). 
The large backscattering yield at high channel numbers (375-425) is caused by the implantation damage in the blistered surface region and the deformation of silicon layers above $\mathrm{H}_{2}$-gas bubbles, whose pressure was not sufficient to cause the "popping off" of the silicon over layers. The peak between channel 345 and 375 is due to the implantation damage in the non-blistered sample surface area. Interestingly, the energy slice of the signal originating from the implantation damage zone in the non-blistered sample area is considerable smaller than that generated by the amorphous layer in the as-implanted sample. The amorphous layer in the as-implanted sample extends from a depth of $145 \mathrm{~nm}$ to $465 \mathrm{~nm}$ and thus has a thickness of $320 \mathrm{~nm}$. The thickness of the implantation damage zone after annealing was measured to be $85 \mathrm{~nm}$. Since the crystalline quality of the silicon over layer in non-blistered sample areas is unknown, the stopping power of the incoming analyzing beam penetrating this silicon over layer is also not known. Therefore, the channeling spectrum of the annealed sample allows only determining the thickness of the buried damage layer, but not its depth. However, the RBS channeling data suggest that during annealing damage recovery took place in the surface near side of the damage zone. It should be noted, that although the backscattering peak of the buried damage layer from the annealed sample does not reach the yield of the randomly aligned RBS spectrum, the damage layer could very well be amorphized. The signal backscattered from blistered surface areas does not contribute to the yield of the buried damage layer and consequently, even amorphized, the buried damage layer would not cause a backscattering peak height, which reaches the yield of the randomly aligned RBS spectrum.

Detailed information about the degree of damage recovery in the shallow part of the annealed sample and the degree of crystal disturbance in the remaining damage layer cannot be deduced by RBS channeling. However, XTEM imaging of the annealed sample allows for a more detailed assessment of the damage recovery during the heat treatment. Figure 5-41 shows a XTEM image of the annealed sample. The image reveals, that during the heat treatment the shallow region of the amorphous layer recrystallized. The electron diffraction patters from this region (not shown) indicates a high crystalline quality of the silicon above the damaged zone. The damaged region ranges from a depth of $394 \mathrm{~nm}$ to $465 \mathrm{~nm}$. 


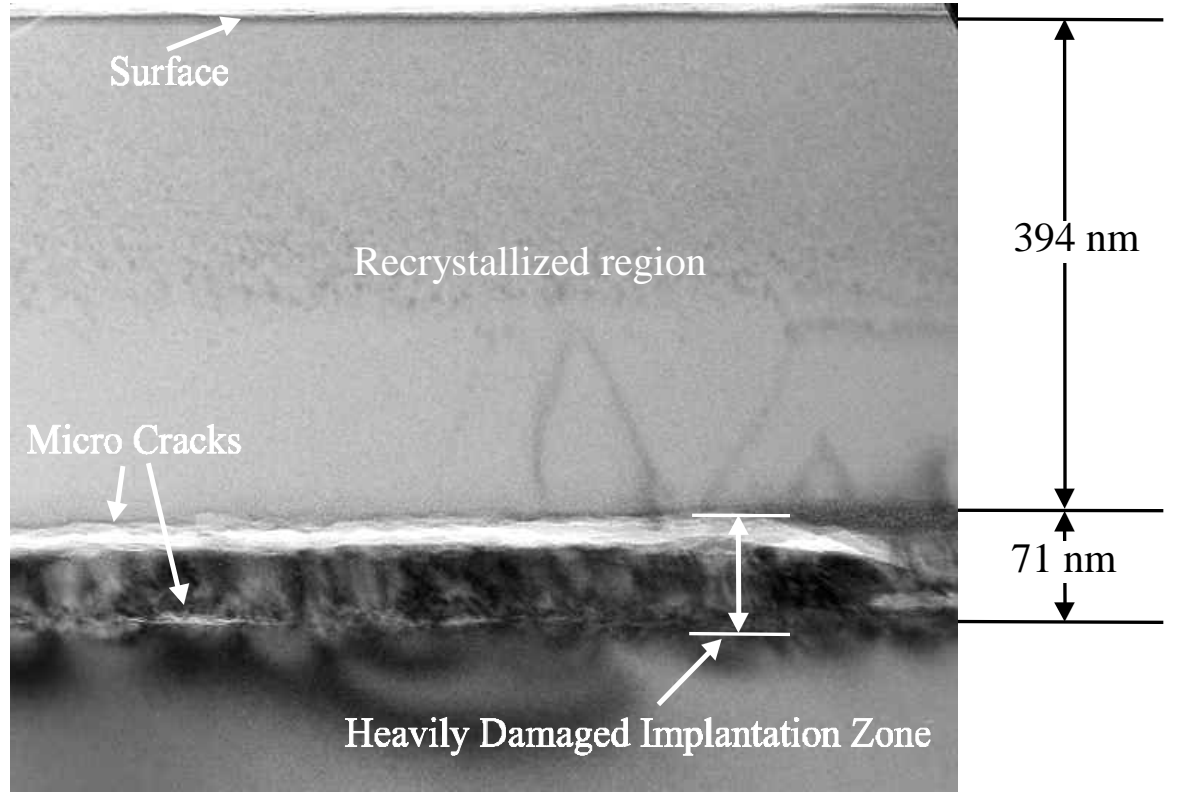

Figure 5-41: Cross-sectional TEM bright-field images from a single crystal $<100\rangle$ silicon wafer, $\mathrm{H}$ ionirradiated under cryogenic conditions with $42 \mathrm{keV} \mathrm{H}$-ions to a dose of $2 \times 10^{17} \mathrm{H} / \mathrm{cm}^{2}$ and subsequently annealed at $600{ }^{\circ} \mathrm{C}$ for 30 minutes, viewed edge on in the [110] silicon projection. The image shows the buried densely damaged implantation zone, permeated with micro cracks at its periphery with two distinct depths of $394 \mathrm{~nm}$ and $465 \mathrm{~nm}$.

Knowing that the shallow part of the damaged zone recrystallized during annealing, it is now possible to determine the depth of the buried damage layer from the RBS channeling spectrum of the annealed sample (figure 5-40 right part). The stopping power for the incoming channeled analyzing beam in non-blistered sample areas can be assumed to be that of channeled He-ions in crystalline Si. Implementation of these stopping power values into the RBS channeling shows that the energy interval, in which the signal from the buried damage layer appears, corresponds to a depth interval between $380 \mathrm{~nm}$ and $465 \mathrm{~nm}$. This is in good agreement with the XTEM observations.

Previous studies have shown, that recrystallization of an amorphous silicon layer by solid phase epitaxy is achievable at the present anneal temperature [149,150]. Due to the high concentration of hydrogen in the implantation zone and its interaction with lattice defects, which prohibits recrystallization of the silicon, only the shallow part of the amorphous layer recrystallized. Further examination of figure 5-41 shows, that micro cracks permeate the silicon wafer at the periphery of the implantation damage zone. Their depths are measured to be $394 \mathrm{~nm}$ for the shallow and $465 \mathrm{~nm}$ for the deep crack location. Comparing these depths with the depth locations of the micro cracks in the 
samples implanted to lower H-doses (418 and $456 \mathrm{~nm}$ ) the XTEM image reveals a shift of the micro crack locations towards a larger and shallower depth, respectively.

Considering the larger thickness of the implantation damage zone compared to the lower H-dose implants and the higher hydrogen concentration at the periphery of the implantation damage zone, the location of the micro cracks are in agreement with the suggested influence of the implantation damage on the Ion-Cut location in terms of the out-of-plane tensile strain and the change of fracture toughness: The implantation zone consists of heavily damaged silicon and therefore the fracture toughness of the material within the implantation zone very high. Furthermore, plastic flow occurring in the heavily damaged silicon [128] reduces the in-plane stress and the corresponding out-ofplane tensile strain, which would facilitate (100) H-platelet formation.

The integrated compressive stress in the implantation zone in the as-implanted sample was calculated by the curvature change of the silicon wafer during the $\mathrm{H}$-ion implantation. The wafer curvature change was measured with the laser scanning setup. The value of the integrated compressive stress in the implantation zone was found to be $-60 \mathrm{MPa}$. This value is quite low and roughly half the value measured for the sample, implanted with $1 \times 10^{17} \mathrm{H} / \mathrm{cm}^{2}$ (see figure 5-36). This confirms the occurrence of strain relieving plastic flow within the damage layer.

On the periphery of the implantation zone however, the amount of crystal damage decreases rapidly, thus making these two depth locations favorable for H-platelet formation: The damage decreases locally to a value, which does not allow for stress relieving plastic flow and thus the in-plane stress reaches high levels at these depths, making them the locations of maximum out-of-plane tensile strain. This is confirmed by earlier measurements of the out-of-plane strain as a function of depth in semiconductor material, which contains an ion-implantation induced amorphous layer. The measurements were performed by means of X-ray double crystal diffractometry. [145] The data revealed sharp peaks in the out-of-plane tensile strain depth distributions at the interfaces between the amorphous layer and the undisturbed crystal. Therefore, I assume that the out-of-plane strain has two maxima at the periphery of the damage layer, two depth locations which coincide with the depths of the observed micro cracks. The high $\mathrm{H}$-implantation dose provides enough hydrogen at the periphery of the damage zone for H-platelet formation.

Finally, it should be noted that although the XTEM image suggests that micro crack formation is more intense at the shallow depth location, AFM measurements of the surface blister depth revealed, that surface blistering takes place at the deep micro crack 
location. Figure 5-42 shows the results of AFM measurements on a surface blister bottom.
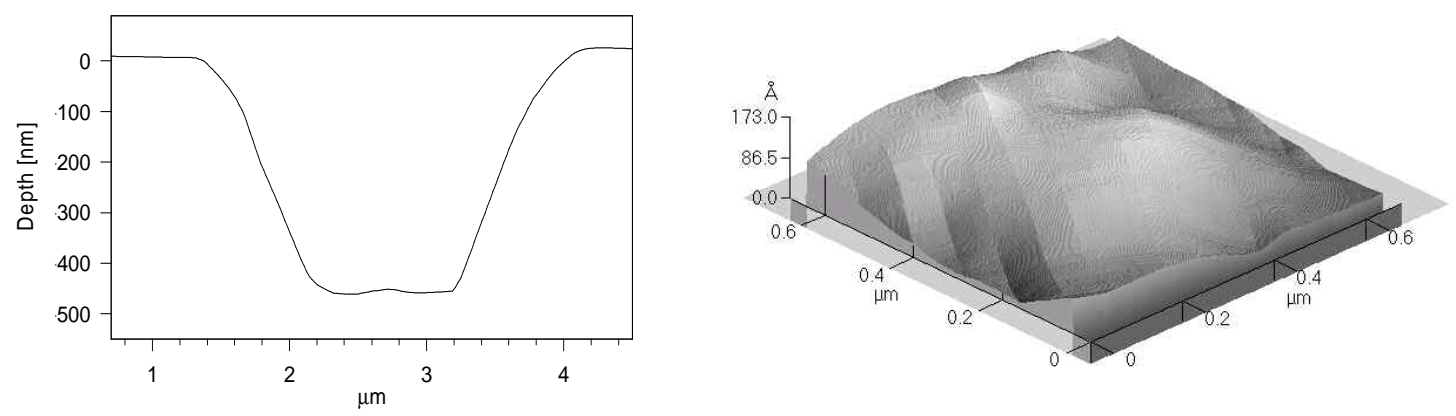

Figure 5-42: AFM trace of a surface blister (left part) and three-dimensional AFM image of the surface blister bottom (right part). The sample was implanted with $40 \mathrm{keV}$ protons to a dose of $2 \times 10^{17} \mathrm{~cm}^{-2}$ and subsequently annealed at $600{ }^{\circ} \mathrm{C}$ for 30 minutes.

The image shows that the crack does not oscillate between the two micro crack depths. AFM measurements of the surface blister depth revealed that the blister depth equals the depth of the deeper micro crack location. It is unclear, whether Ion-Cut occurs only at the deep micro crack location or at both micro crack depths. 


\subsection{Influence of the Sample Treatment on Ion-Cut Location}

The samples studied in chapter 5.2 were all implanted with protons at the liquid nitrogen temperature of $77 \mathrm{~K}$ and an implantation energy of $42 \mathrm{keV}$. To ensure that the conclusion about the mechanisms of the Ion-Cut, drawn from these observations, are correct, the influences of implantation temperature and energy on the Ion-Cut location relative to the $\mathrm{H}$-concentration and the implantation damage depth distribution must be evaluated. It will also be important to assess the role of the annealing temperature or whether the presence of a bonded stiffener can influence the ion-cutting mechanisms.

The influence of the implantation temperature and energy on the Ion-Cut location was examined using low H-dose implantation experiments. Figure 5-43 plots the Ion-Cut depths and $\mathrm{H}$-ion irradiation induced damage distributions for the samples implanted with $42 \mathrm{keV} \mathrm{H}$-ions to a dose of $5 \times 10^{16} \mathrm{~cm}^{-2}$ at cryogenic temperatures and the sample, implanted with $175 \mathrm{keV} \mathrm{H}$-ions to the same dose, but at room temperature.

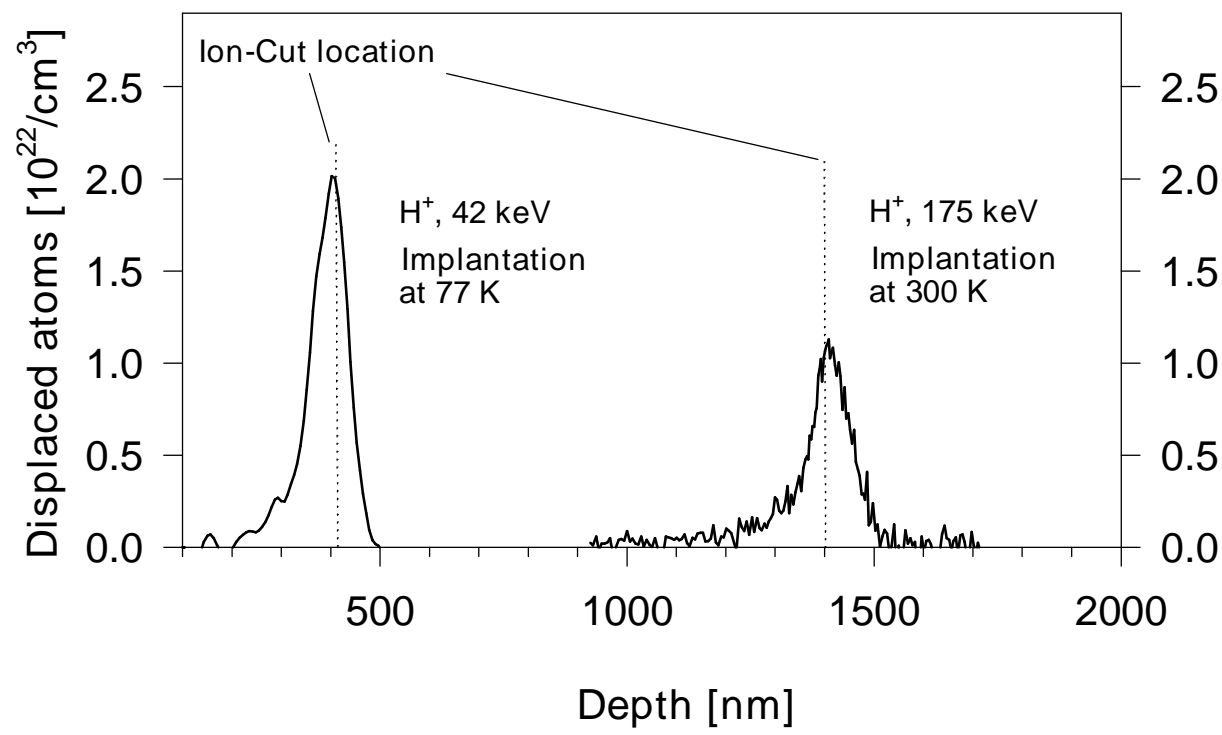

Figure 5-43: As-implanted implantation damage depth distribution and Ion-Cut location for the silicon samples, implanted with $42 \mathrm{keV} \mathrm{H}$-ions at liquid nitrogen temperature and with $175 \mathrm{keV} \mathrm{H}$-ions at room temperature. The implantation dose was $5 \times 10^{16} \mathrm{H} / \mathrm{cm}^{2}$ for both samples. The data are from figure 5-3 and $5-.23$, respectively. 
A comparison of the data from the two samples shows that for both cases the Ion-Cut location is well correlated with the damage peak. This suggests that the correlation between cut depth and damaged location exists for a wide range of ion energies and implantation temperatures. This observation strengthens the hypotheses, made in chapter 5.1.5. As long as the ion-irradiation induced lattice damage is comparable in amount, the out-of-plane tensile strain in the damaged depth region enhances (100) $\mathrm{H}$ platelet formation, independent of the implantation temperature. It should be noted, that for the sample implanted with $175 \mathrm{keV}$ protons, ion-cutting occurred not in the form of surface blistering, but complete delamination of the exfoliated layer after bonding of the $\mathrm{H}$-implanted sample to a stiffener. Thus, the depth where H-platelets and micro cracks form is apparently independent of the presence of a bonded stiffener for silicon samples implanted with $5 \times 10^{16} \mathrm{H} / \mathrm{cm}^{2}$.

To investigate the influence of the implantation temperature, the implantation energy, and the presence of a bonded stiffener on the Ion-Cut location in high $\mathrm{H}$-dose implanted silicon samples, an Ion-Cut sample with an implantation dose of $1 \times 10^{17} \mathrm{H} / \mathrm{cm}^{2}$ was produced. The treatment the sample underwent differed from the $1 \times 10^{17} \mathrm{H} / \mathrm{cm}^{2}$ implant sample, studied in chapter 5.2.

For this experiment a $<100>$ p-type silicon wafer with a resistivity of $1-10 \Omega \mathrm{cm}$ (the same substrate used in chapter 5.2) was implanted with $100 \mathrm{keV} \mathrm{H}_{2}{ }^{+}$ions to a dose of $5 \times 10^{16} \mathrm{H} / \mathrm{cm}^{2}$. Because of the $\mathrm{H}_{2}{ }^{+}$fragmentation at the surface this implantation process is equal to the implantation of atomic $\mathrm{H}$-ions at $50 \mathrm{keV}$ to a dose of $1 \times 10^{17} \mathrm{H} / \mathrm{cm}^{2}$. The implantation was carried out at room temperature. The H-beam current was about $1 \mu \mathrm{A} / \mathrm{cm}^{2}$, keeping the ion beam heating effect in the sample moderate. After the $\mathrm{H}_{2}$-ion implantation, the sample was bonded to a stiffener, a silicon wafer capped with a $0.5 \mu \mathrm{m}$ thick $\mathrm{SiO}_{2}$ layer. Prior to the hydrophilic bonding both wafers underwent a standard RCA clean. To achieve strong chemical bonds between the two wafers the bonded pair was further annealed at a temperature of $100{ }^{\circ} \mathrm{C}$ for 2 hours + $250{ }^{\circ} \mathrm{C}$ for 30 minutes.

The two bonded wafers were cut into three pieces. Ion-cutting of the samples was achieved with two different methods. The wafers were annealed at either $350{ }^{\circ} \mathrm{C}$ or $600{ }^{\circ} \mathrm{C}$, respectively. The complete delamination of the hydrogen implanted wafer was achieved after an anneal duration of 11 minutes for the anneal temperature of $350{ }^{\circ} \mathrm{C}$ and after 75 seconds for the anneal temperature of $600{ }^{\circ} \mathrm{C}$. 
For the third sample, Ion-Cut was induced in a mechanical way by a recently developed method [151]: A razorblade was inserted sidewise into the bonded wafers near the bond interface. Since the H-implantation dose is fairly high and the H-ion implantation was carried out at room temperature instead of cryogenic temperatures, H-platelets formed already in the as-implanted sample. The low bond strength of the $\mathrm{Si}-\mathrm{H}-\mathrm{H}-\mathrm{Si}$ platelets leads to a reduction of the fracture strength in this area. Thus, the high dose H-ion implantation causes the implantation zone to be weaker than the bond interface and therefore the crack that results from the insertion of a razorblade preferentially propagates along the implantation zone instead at the bond interface.

Figure 5-44 plots the RBS channeling spectrum obtained from the H-implanted sample in the as-implanted state and the corresponding damage depth distribution deduced from the spectrum by the method described in chapter 4.2.1.

a)

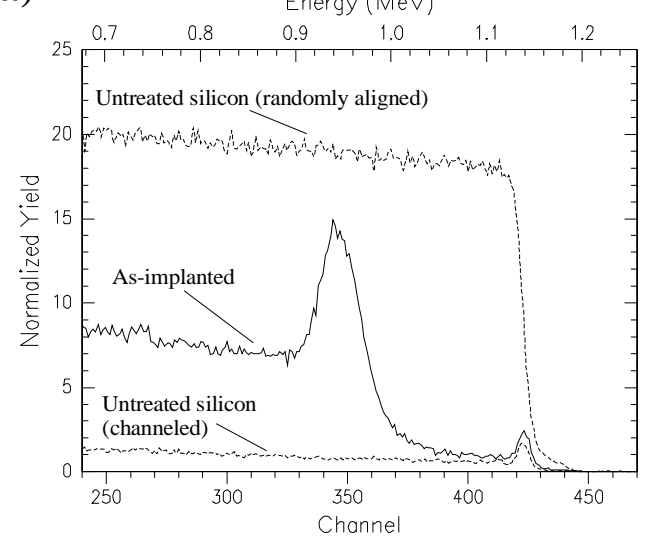

b)

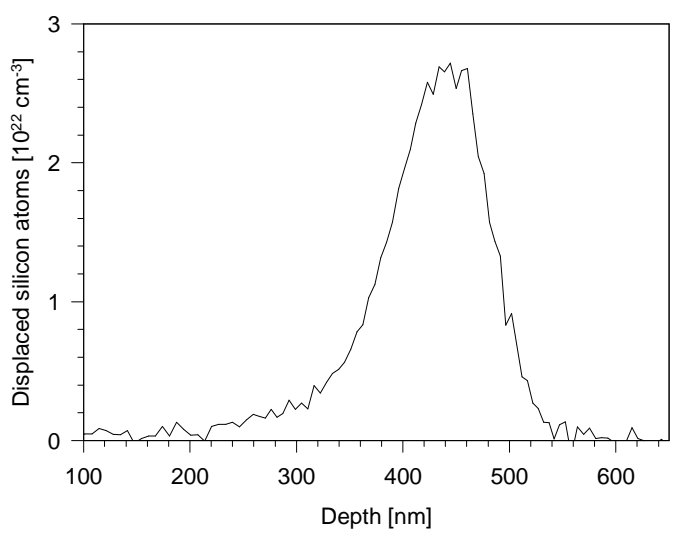

Figure 5-44: (a) $2.0 \mathrm{MeV}$ He RBS-channeling spectrum of the <100> Si wafer, implanted with $100 \mathrm{keV}$ $\mathrm{H}_{2}$-ions to a dose of $5 \times 10^{16} \mathrm{~cm}^{-2}$. Also shown for comparison are a random spectrum and the channeling spectrum of untreated silicon. (b) In-depth distribution of the implantation damage concentration, deduced from the RBS channelling spectrum.

The density of displaced silicon atoms in the damage layer is about $30 \%$ lower than in the sample implanted with $42 \mathrm{keV}$ protons at liquid nitrogen temperature to the same $\mathrm{H}$ dose and even $18 \%$ lower than in the sample implanted with $42 \mathrm{keV}$ protons at liquid nitrogen temperature to a dose of $7 \times 10^{16} \mathrm{H} / \mathrm{cm}^{2}$ (see figure 5-35, p. 105). This is due to the implantation taking place at room temperature, which enables partial damage recovery during the ion-implantation process. The depth distribution of the implantation damage deduced from the RBS channeling spectrum is presented in figure 5-44 (b). The damage peak is measured to be located at a depth of $435 \mathrm{~nm}$.

Figure 5-45 shows the ERD spectrum from the as-implanted sample and the Hconcentration depth distribution deduced from the ERD analysis. The data were analyzed 
as described in chapter 4.1.2. The result of the ERD analysis, presented in figure 5-45 (b), reveals, that diffusion of the H-implant due to beam heating effects is not observed. The shape of the H-depth profile appears to be comparable to the one resulting from $\mathrm{H}$-ion implantations to lower energies at cryogenic temperatures (see fig. 5-25). The depth of maximum H-concentration is measured to be $465 \mathrm{~nm}, 30 \mathrm{~nm}$ deeper than the implantation damage peak.
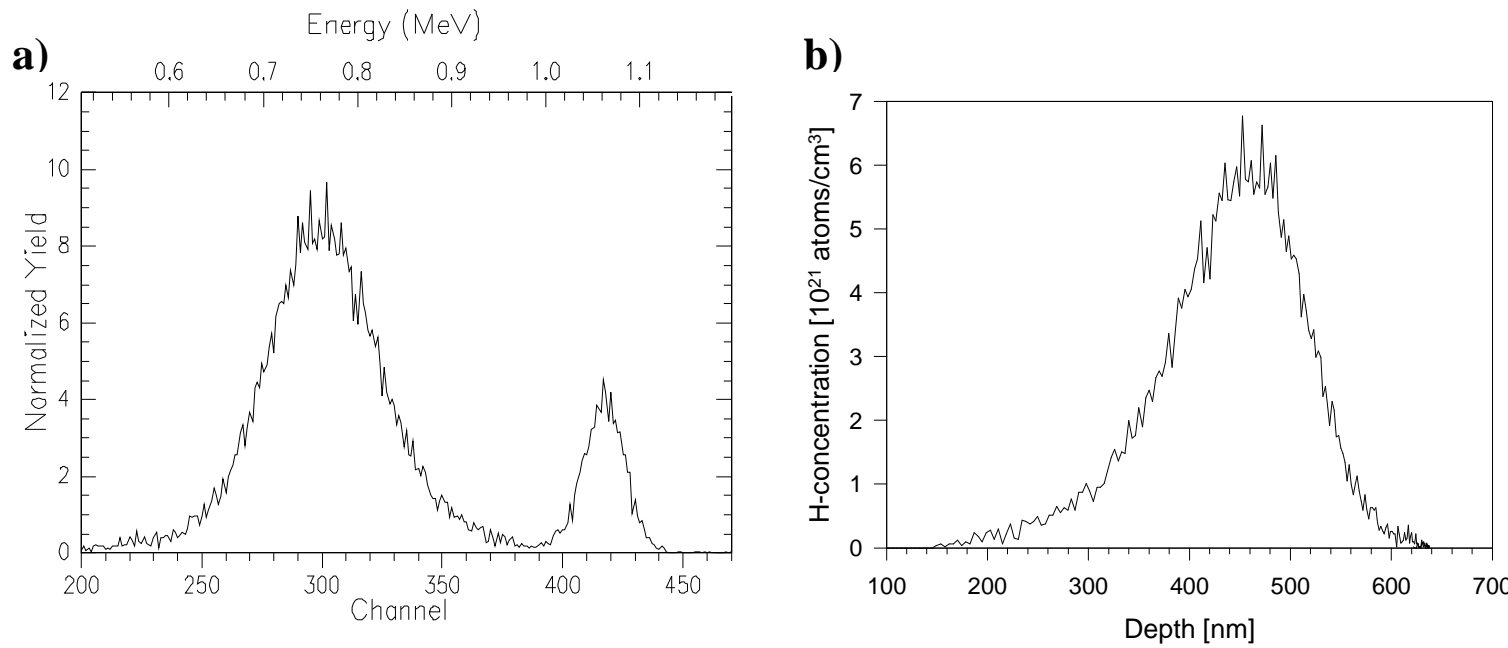

Figure 5-45: (a) $3.0 \mathrm{MeV} \mathrm{He} \mathrm{ERD}$ spectrum of the $\mathrm{H}$-implanted $<100\rangle \mathrm{Si}$ wafer, implanted with $100 \mathrm{keV}$ $\mathrm{H}_{2}$-ions to a dose of $5 \times 10^{16} \mathrm{~cm}^{-2}$ at room temperature. (b) In-depth distribution of the hydrogen concentration deduced from the ERD spectrum.

As mentioned above, after H-implantation and bonding, ion-cutting was induced by annealing at two different temperatures and mechanically by the insertion of a razorblade near the bond interface. After the Ion-Cut, the samples were analyzed by RBS in random and channeling mode, ERD analysis, XSEM, AFM, and XTEM.

RBS channeling spectra of the samples after ion-cutting are shown in figure 5-46. The RBS channeling spectra of the samples, which underwent different heat treatments, overlay perfectly. Therefore, only one set of spectra is shown. The data reveal a far larger amount of damage remaining in the exfoliated layer compared to the damage amount in the donor wafer. This observation was made for all samples, i.e. for mechanically and thermally cut samples. This indicates that the Ion-Cut took place at the end-of-range of the implantation zone, independent on the method the Ion-Cut was provoked. This hypothesis is strengthened by the fact that the surface peaks of the buried silicon dioxide layers in the RBS channeling spectra from the exfoliated layers appear at the same energy of $0.925 \mathrm{MeV}$ (channel 338). 


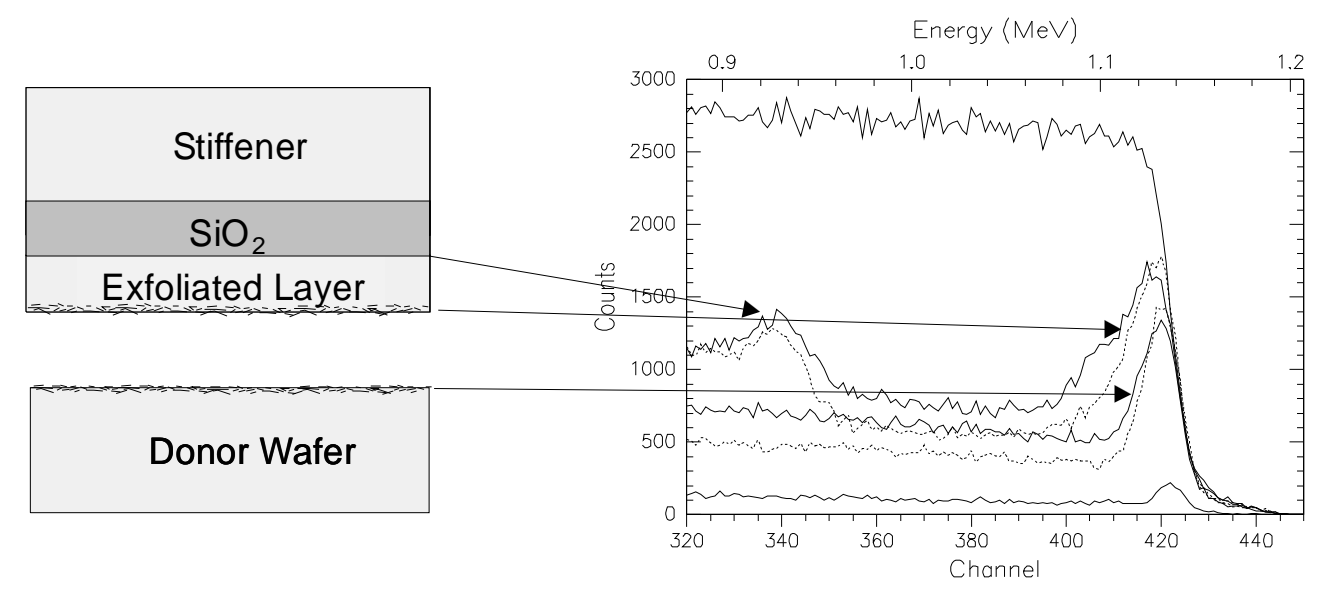

Figure 5-46: 2.0 MeV He RBS-channeling spectra of the donor wafer and the exfoliated layer, channelled along the $\langle 100\rangle$ direction. The Ion-Cut was induced either mechanically (-------) or by sample annealing at $350^{\circ} \mathrm{C}$ for 11 minutes (—). For comparison the spectrum of untreated silicon in random alignment and channeling condition are shown, too (thin solid lines).

Examining the RBS channeling spectra in more detail, the data indicate a higher amount of displaced silicon atoms in the donor wafer and the exfoliated layer of the sample, IonCut by annealing. Also, the dechanneling yield (channel $350-390$ ) is higher for these samples. This effect can be explained, considering the rearrangement of the H-implant during the heat treatment into (among other complexes) $\mathrm{H}_{2}$ molecules, residing in the silicon lattice. These hydrogen complexes have been shown to induce a considerable amount of the displaced silicon atoms in the implantation zone. [42] Therefore, the samples, cut by thermal annealing, contain more lattice damage than the mechanically cut samples due to the thermally activated $\mathrm{H}$-defect evolution in the implantation zone.

RBS measurements on the exfoliated layers were also performed in random alignment of the analyzing beam. Figure 5-47 plots as an example the RBS spectrum obtained from the exfoliated layer, cut by annealing at $350{ }^{\circ} \mathrm{C}$ for 11 minutes. The signal between channel 240 and 420 originates from the exfoliated silicon layer, whereas the lower signal at lower channel numbers is generated by $\alpha$-particles, backscattered from the silicon atoms in the $\mathrm{SiO}_{2}$ layer. The lower concentration of silicon atoms in the $\mathrm{SiO}_{2}$ layer consequently creates a lower backscattering yield. Since the interface between the exfoliated layer and the silicon dioxide layer can be detected easily by RBS, this method provides a tool for the thickness measurements of the exfoliated layer. The simulation of the spectrum measures the average thickness of the exfoliated layer to be $483 \mathrm{~nm}$. Due to the surface roughness of the exfoliated layer and the resulting deviation of the layer thickness, the Si signal does not drop sharply at channel 240. Energy straggling could not account for the energy spread at channel 240 . The backscattered ion energy at the 
point where the silicon signal starts to decrease corresponds to a layer thickness of $475 \mathrm{~nm}$. The energy at which the signal reaches the lower plateau of the $\mathrm{SiO}_{2}$ layer signal corresponds to a thickness of $500 \mathrm{~nm}$.

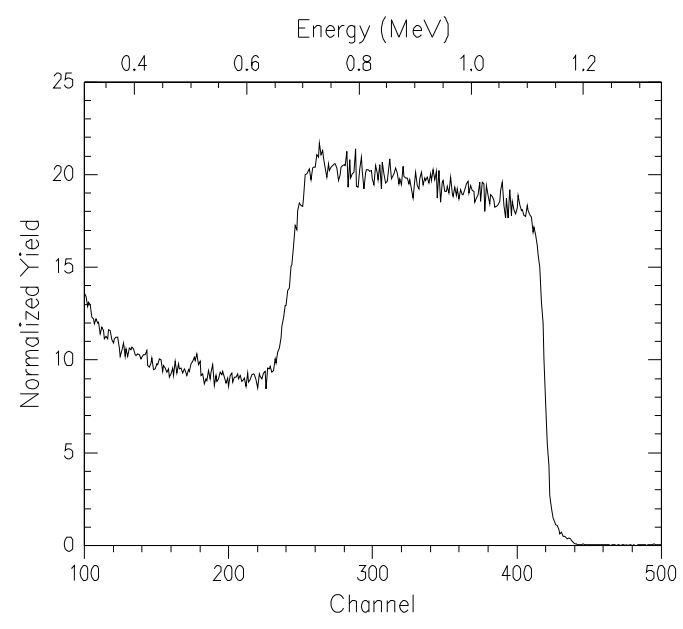

Figure 5-47: $2.0 \mathrm{MeV}$ He RBS spectrum of the exfoliated layer. The Ion-Cut was induced by annealing at $350{ }^{\circ} \mathrm{C}$ for 11 minutes. The sample was aligned 60 degrees to the analyzing beam in order to obtain a higher accuracy in the depth measurement.

The RBS spectra of the samples, which were cut mechanically or by annealing at $600{ }^{\circ} \mathrm{C}$ for 75 seconds overlay perfectly with the RBS spectrum shown in figure 5-47. This is a further indication that the depth location of cleavage is independent on the method of ion-cutting.

The thickness of the exfoliated layer was also measured by XSEM. Figure 5-48 shows as an example the XSEM image obtained from the sample, cut mechanically by the razorblade insertion method. The exfoliated layer was measured to be $478 \mathrm{~nm}$ thick for the mechanically and thermally ion-cut samples, in good agreement with the RBS measurements.

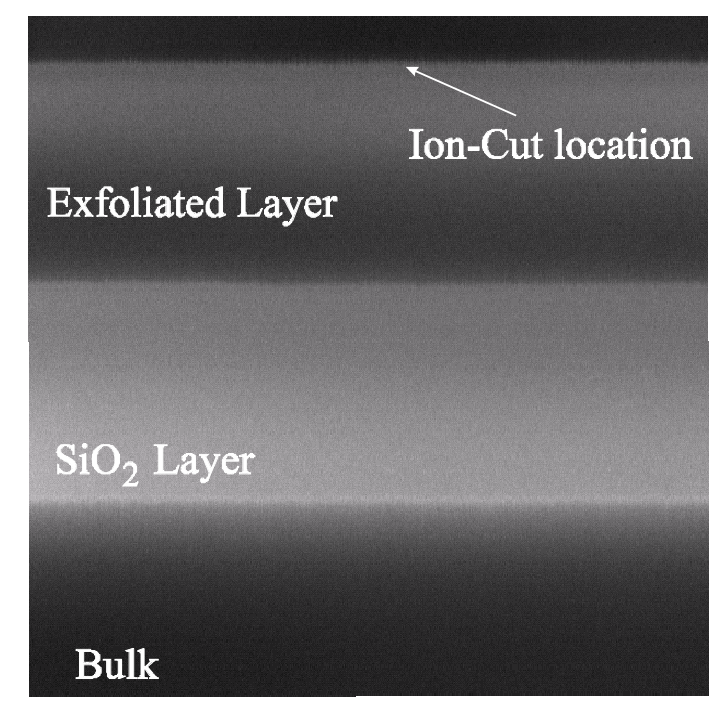

Figure 5-48: Cross-section SEM image of the exfoliated silicon surface layer after bonding and ion-cutting. The Ion-Cut was induced mechanically. 
The implanted hydrogen profile after the Ion-Cut was monitored by ERD, using a 3.0 $\mathrm{MeV} \mathrm{He}$ analyzing beam. The measurements were carried out to obtain quantitative information about the $\mathrm{H}$-content remaining in the donor wafers and the exfoliated layers. Figure 5-49 presents the ERD spectra taken from the exfoliated layer and the donor wafer.

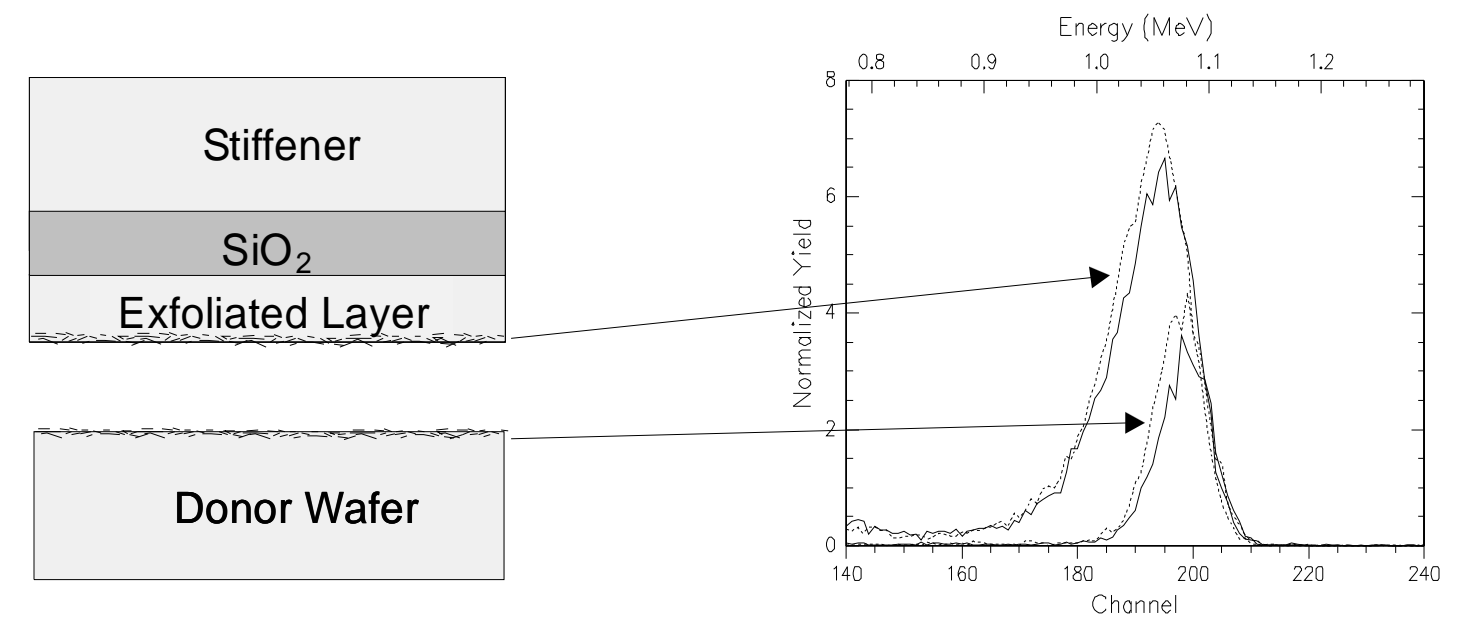

Figure 5-49: $3.0 \mathrm{MeV}$ He ERD spectra of the donor wafer and the exfoliated layer. The Ion-Cut was induced either mechanically (-------) or by sample annealing at $350{ }^{\circ} \mathrm{C}$ for 11 minutes (- - ).

As mentioned earlier, the energy straggling in the ERD spectra prevents a distinction between the hydrogen from the H-implant and the hydrogen representing surface contaminations. Thus, the information deduced from the ERD spectra provides only a rough estimate of the ratio between H-content remaining in the exfoliated layer and the donor wafer. As can be seen clearly, the amount of hydrogen in the exfoliated layer is substantially higher than the one remaining in the donor wafer. The detected $\mathrm{H}$-amount in the exfoliated layer is 3.1 times higher than in the donor wafer, independent on the method of ion-cutting. Considering the overlap of the H-implant signal with the $\mathrm{H}$ surface contamination peak, the ratio between the $\mathrm{H}$-content in the exfoliated and the $\mathrm{H}$ content in the donor wafer is apparently even higher. This result is consistent with the measured H-concentration depth profile (see figure 5-45 (b)) and the depth of the IonCut. Cleavage occurs in the end of tail region in the implantation zone, leaving significantly more hydrogen in the exfoliated layer than in the donor wafer.

The ERD spectra obtained from the sample, which was annealed at $600{ }^{\circ} \mathrm{C}$ for 75 seconds overlap perfectly with the ERD spectra of the sample annealed at $350{ }^{\circ} \mathrm{C}$ for 11 minutes and are therefore not shown. Examining the ERD spectra further, it seems as if more hydrogen remains in the mechanically cut samples compared to the samples, cleaved by annealing. This is consistent with the fact that in mechanically induced ioncutting the cut propagates along H-platelets and is caused by the low surface energy of 
the H-decorated platelets, whereas in the heat treatment induced Ion-Cut the high internal pressure in $\mathrm{H}_{2}$-gas bubbles initiate the fracture, which in turn leads to the release of a large amount of $\mathrm{H}_{2}$-gas at the time of fracture. However, the uncertainty of the surface peak height does not allow for the determination of the absolute amount of the remaining H-implant.

Finally, it should be noted, that the ERD spectra indicate, that no noticeable diffusion of the $\mathrm{H}$ into the bulk of the material or towards the bond interface occurred. The shapes of the $\mathrm{H}$ depth profiles are similar, independent on the Ion-Cut method for both the exfoliated layer and the donor wafer.

To obtain detailed information about the micro structure of the implantation zone, XTEM imaging of the bonded and the Ion-Cut samples was performed. The left part of figure 550 shows a low magnification image of the bonded samples. The image shows a heavily damaged layer, which extends from a depth of $335 \mathrm{~nm}$ to $535 \mathrm{~nm}$, in agreement with the RBS channeling analysis (see figure 5-44 (b)). Platelets aligned along $\{111\}$ planes appear in the non-damaged material beyond the damaged depth region up to

After bonding

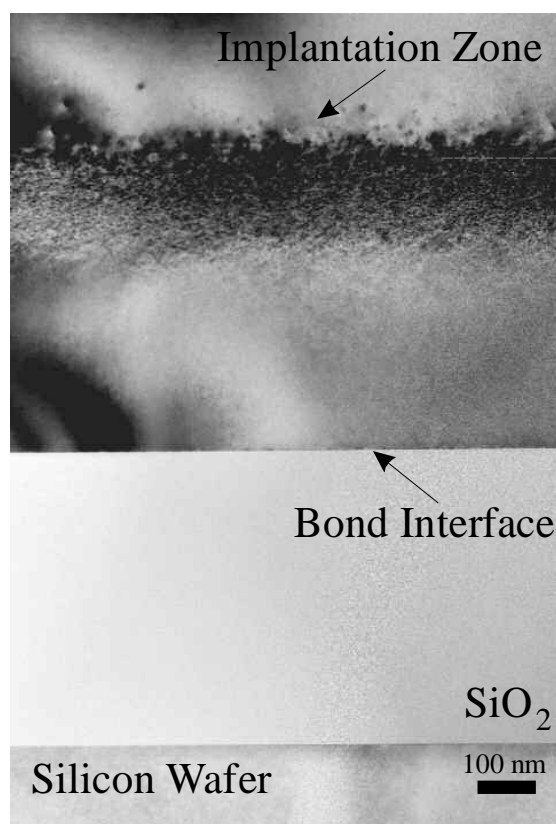

After mechanical ion-cutting

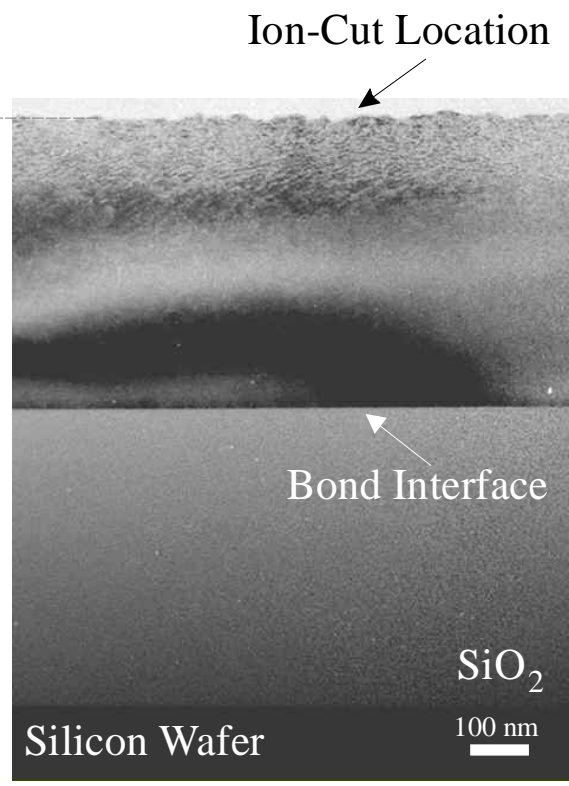

Figure 5-50: Low magnification bright field cross-section TEM images of the bonded wafers and after mechanical induced ion-cutting. The samples were viewed edge on in the [110] silicon projection.

a depth of $615 \mathrm{~nm}$. Since the bonded sample underwent a moderate thermal anneal at $250{ }^{\circ} \mathrm{C}$ for one hour, $\mathrm{H}$-platelet formation in the end-of-tail region in the $\mathrm{H}$-concentration 
depth profile is expected. Assuming these platelets to be hydrogen decorated or filled, this observation is in accordance with the ERD analysis of the as-implanted sample, which showed the H-concentration depth distribution to extend to a depth of $640 \mathrm{~nm}$.

The right part of figure 5-50 presents a low magnification image of the bonded wafers after cleavage by the insertion of a razorblade. The image shows clearly, that the cut takes place at the end of the implantation zone. The main part of the H-platelet filled damage region remains in the exfoliated layer. The location of the Ion-Cut ranges from a depth of $476 \mathrm{~nm}$ to $492 \mathrm{~nm}$. This measurement is in good agreement with the RBS analysis of the exfoliated layer, which showed the Ion-Cut to occur at a depth range extending from $475 \mathrm{~nm}$ to $500 \mathrm{~nm}$.

Higher magnification XTEM images of the implantation zone after the Ion-Cut are displayed in figure 5-51. Cleavage of the H-implanted wafer was either provoked mechanically or by annealing at $350{ }^{\circ} \mathrm{C}$ for 11 minutes.

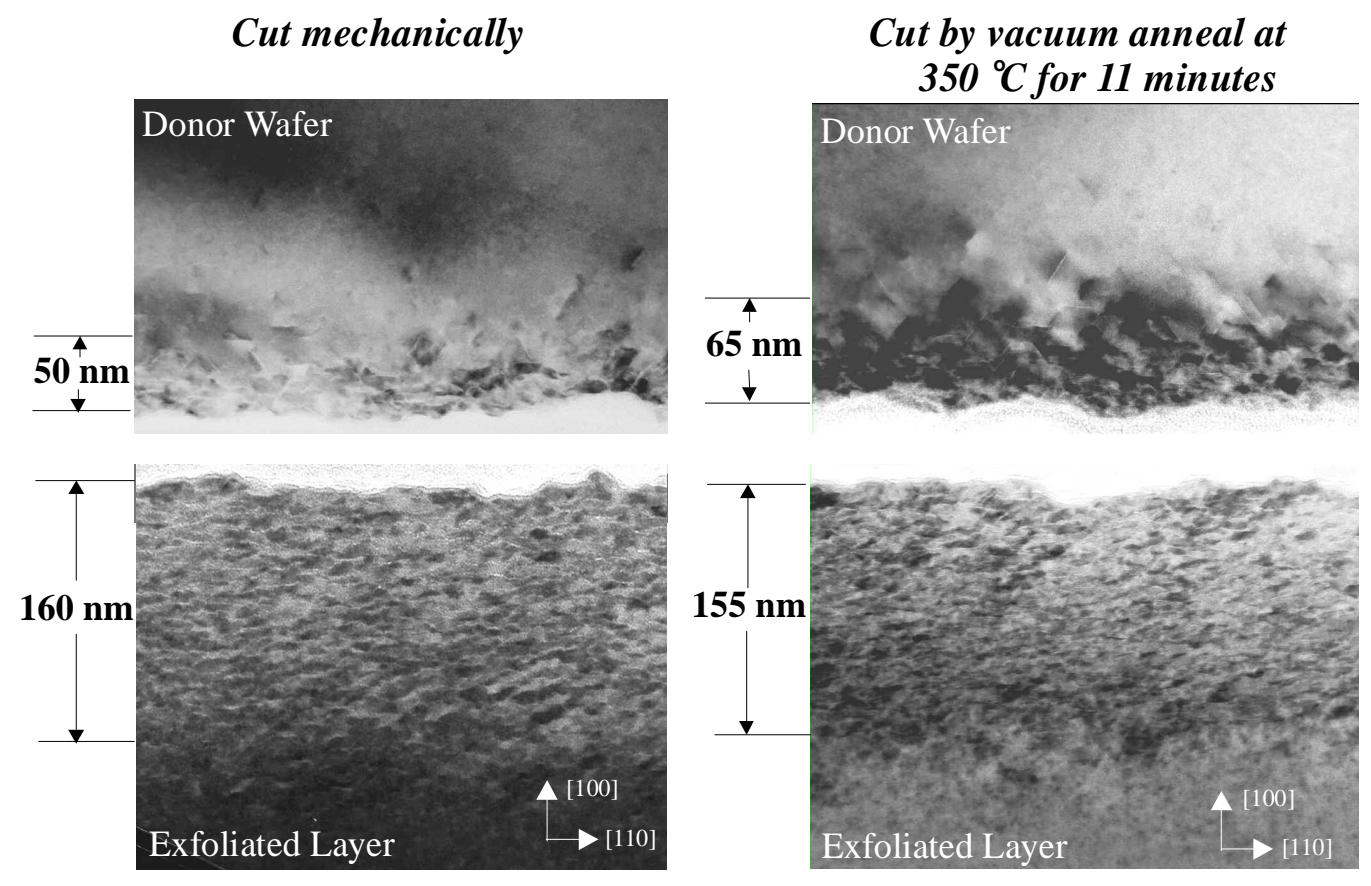

Figure 5-51: High magnification bright field cross-section TEM images of donor wafer and the exfoliated layer after mechanically and thermally induced cleavage, respectively. The samples were viewed edge on in the [110] silicon projection.

Unlike in the samples studied in chapter 5.2, which underwent an anneal at $600{ }^{\circ} \mathrm{C}$ for 30 minutes, H-platelets are still present after the heat treatment. This is due to the far lower annealing temperature and duration. The Ostwald ripening process, making larger $\mathrm{H}$ platelets $/ \mathrm{H}_{2}$-gas bubbles grow on the expense of smaller ones, is not as far developed as in the high temperature annealed samples studied in chapter 5.2. (100) H-platelets are 
still present in the exfoliated layer and the shallow part of the donor wafer. Also, Hplatelets aligned along $\{111\}$ planes are still present in the end-of-tail region of the $\mathrm{H}$ concentration depth distribution, i. e. the sub surface region in the donor wafer. Comparing the XTEM images of the donor wafers, more <111> platelets are observed in the sample cut by thermal heat treatment. This indicates that the heat treatment during the wafer bonding was not sufficient to reach the maximum in H-platelet density. Although a difference in the platelet density in the exfoliated layers is not visible, detailed analysis of higher magnification XTEM images (not shown) revealed, that the $\mathrm{H}$ platelet density in the exfoliated layer and the donor wafer is almost twice as high for the sample cut thermally by annealing at $350{ }^{\circ} \mathrm{C}$ for 11 minutes than in the mechanically cut sample.

The XTEM observation confirms above analysis of the split samples. The IonCut took place in the end-of-tail region of the H-platelet depth distribution. The depth region in which H-platelets appear is about three times as thick in the exfoliated layer as it is in the donor wafer, independent on the method the Ion-Cut was induced.

The mechanically induced Ion-Cut is expected to occur at the depth of maximum H-platelet density, since this depth is the weakest location in terms of fracture toughness. The inner walls of the H-platelets are decorated with H-atoms. Therefore, only the weak Van der Waals interactions between the $\mathrm{H}$ atoms keep the $\mathrm{H}$-platelets from cleavage. On the other hand, the thermal induced Ion-Cut is expected to take place in the depth region of maximum density of highly pressurized $\mathrm{H}_{2}$-gas bubbles, since the pressure in the bubbles supplies the force for the crack propagation throughout the $\mathrm{H}$-implanted silicon wafer. The above results showed, that when silicon is implanted to $1 \times 10^{17} \mathrm{H} / \mathrm{cm}^{2}$, the mechanically and thermally induced Ion-Cut occurs at the same depth.

The similar cut location for mechanically and thermally sliced samples suggests, that the depth of maximum platelet density after bonding will also be the depth of maximum $\mathrm{H}_{2}$-gas bubble density after further annealing. This is in agreement with the proposed influence of the out-of-plane tensile strain on the rearrangement of H-atoms. The out-of-plane tensile strain facilitates the nucleation of H-platelets. These platelets comprise H-bubble precursors. Therefore, as soon as out-of-plane tensile strain evolves, $\mathrm{H}$-platelets nucleate and grow into $\mathrm{H}_{2}$-gas bubbles via the Ostwald ripening mechanism. The region of highest H-platelet density will evolve initially during annealing into the region of largest $\mathrm{H}$-platelets and upon further heat treatment will be the sink for diffusing $\mathrm{H}$-atoms, becoming finally the region of highest $\mathrm{H}_{2}$-gas bubble concentration.

It should be noted, that $\mathrm{H}_{2}$-gas bubbles could be observed in Ion-Cut samples by XTEM imaging: The thickness of the XTEM samples near the sample surface, i. e. in this 
case the Ion-Cut location, is smaller than the $\mathrm{H}_{2}$-gas bubbles radii. Consequently, the $\mathrm{H}_{2}$ gas will no longer be trapped in the bubbles. The release of the $\mathrm{H}_{2}$-gas leads the relaxation of the deformed silicon around the bubbles, leaving behind H-decorated platelets in the XTEM sample.

XTEM imaging was also performed on the sample, Ion-Cut by thermal annealing at $600{ }^{\circ} \mathrm{C}$ for 75 seconds (not shown). The platelet depth distribution of the samples annealed at either $350{ }^{\circ} \mathrm{C}$ for 11 minutes or at $600{ }^{\circ} \mathrm{C}$ for 75 seconds did not show any noticeable differences.

AFM measurements of the sample surfaces after the Ion-Cut were performed on all samples. Figure 5-52 shows as an example the surface of the exfoliated layer after ion-cutting by annealing at $600{ }^{\circ} \mathrm{C}$ for 75 seconds. The rms roughness values of the ioncut surfaces were measured to be $4.9 \mathrm{~nm}$ for the sample cut mechanically, $4.8 \mathrm{~nm}$ for the sample cut by annealing at $600{ }^{\circ} \mathrm{C}$ for 75 seconds, and $4.5 \mathrm{~nm}$ for the sample cut by annealing at $350{ }^{\circ} \mathrm{C}$ for 11 minutes. These values are almost equal, indicating that the method of ion-cutting does not influence the surface roughness of the exfoliated layers.

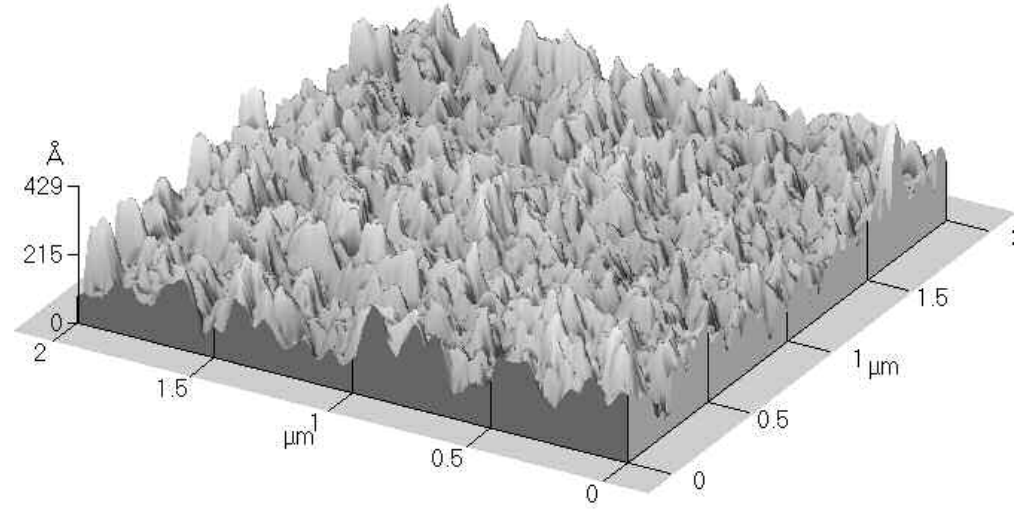

Figure 5-52: AFM image of the silicon surface of the exfoliated layer after cleavage. The Ion-Cut was induced thermally by annealing for 75 seconds at $600{ }^{\circ} \mathrm{C}$.

As mentioned above, the cleavage in the mechanically ion-cut sample is expected to take place in the depth region of maximum H-platelet density. To verify this assumption, a higher magnification XTEM image of the implantation zone was acquired after the sample was bonded to the stiffener. In this state, the sample represents the state it was in before the razorblade insertion, which provokes the crack propagation throughout the wafer. Figure 5-53 shows the obtained XTEM image. 


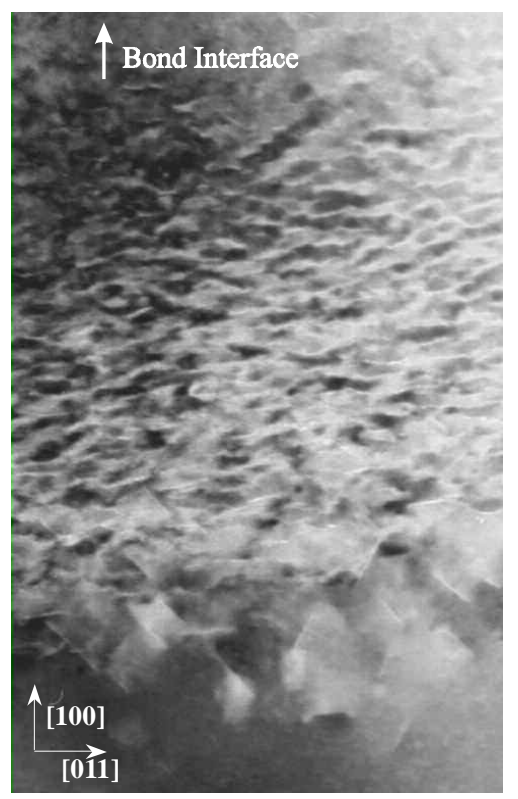

Figure 5-53: Higher resolution bright field XTEM image of the implantation zone of the $\mathrm{H}$ implanted and bonded wafer (see left part of figure 5-50)

The image shows a high density of (100) H-platelets throughout the implantation zone. At the end-of-tail region of the implantation zone, where the damage is low, but the $\mathrm{H}$ concentration still high enough for H-platelet formation, H-platelets are oriented predominantly along $\{111\}$ planes.

The depth distribution of the platelet density in the bonded sample was deduced from TEM images similar to that in figure 5-53. To obtain better statistics the regions in which the platelets where counted were expanded to be three times as big as the sample area shown in figure 5-53. The result of the analysis is presented in figure 5-54. The graph shows H-platelet formation over a wide depth interval, ranging from a depth of $330 \mathrm{~nm}$ to $615 \mathrm{~nm}$.

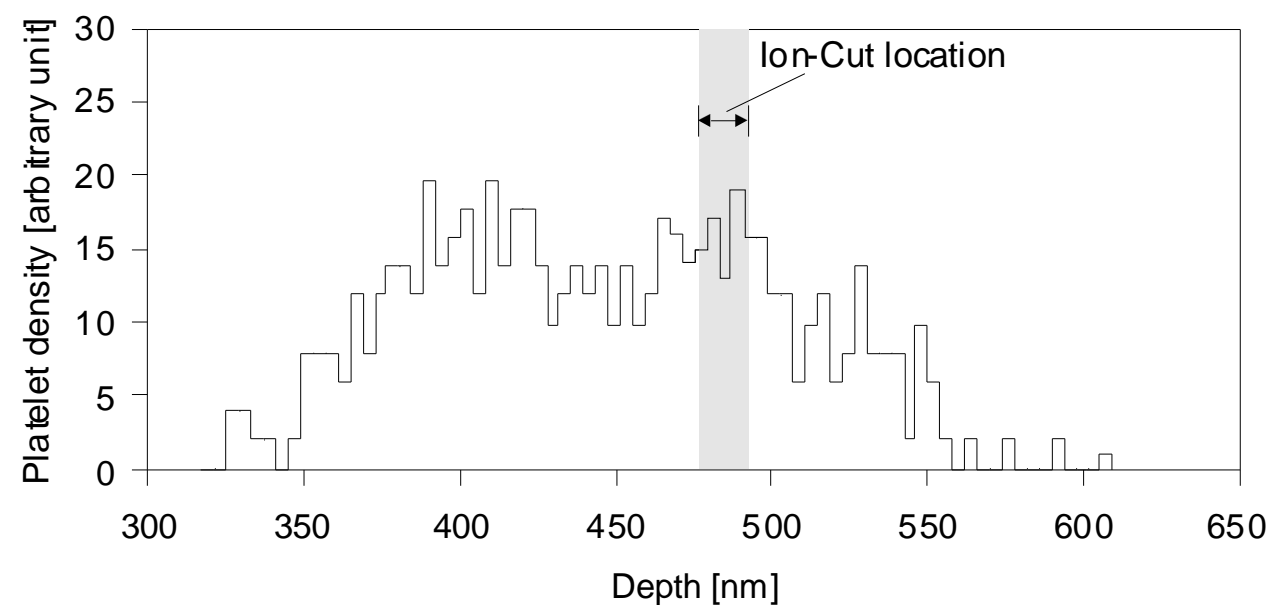

Figure 5-54: Depth distribution of the H-platelet density in the bonded sample. The gray area indicates the depth interval the Ion-Cut took place. 
Interestingly, the plot shows two depth regions of maximum H-platelet density. One has a width of $35 \mathrm{~nm}$ and is centered around a depth of $400 \mathrm{~nm}$. The other one is located in the depth region between $465 \mathrm{~nm}$ and $500 \mathrm{~nm}$ and overlaps with the depth region, where ion-cutting takes place. The plot confirms the hypothesis that the mechanically induced Ion-Cut takes place at the depth of high H-platelet density. To investigate the appearance of two depth regions with maximum H-platelet density, figure 5-55 provides a comparison of the H-platelet density depth distribution with the implantation damage and $\mathrm{H}$-concentration depth profile in the as-implanted sample, respectively.

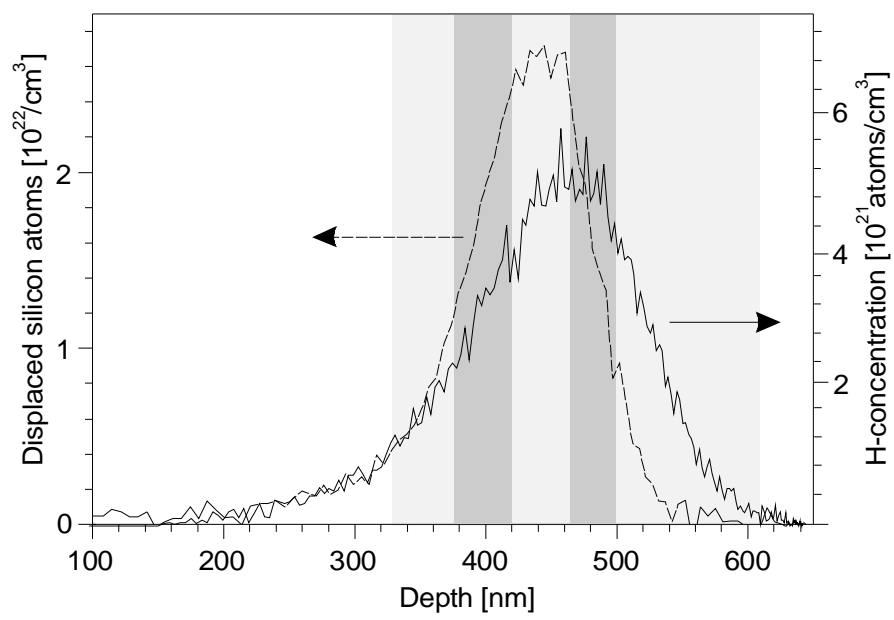

Figure 5-55: Depth distribution of the implantation damage (-- -) and the H-concentration (-) in the sample, implanted at room temperature with $100 \mathrm{keV} \mathrm{H}$-ions to a dose of $5 \times 10^{16} \mathrm{~cm}^{-2}$. The light gray area indicates the depth region where H-platelets appear after the bonding process. The dark gray areas indicate the regions of maximum H-platelet densities.

The graph shows that the highest H-platelet density is reached at the periphery of the depth region of highest damage, but not at the damage peak itself.

Recollecting the results gained on the sample implanted with $42 \mathrm{keV} \mathrm{H}$-ions at cryogenic temperatures, ion-cutting occurred at a depth slightly larger than the hydrogen concentration peak when implanted to $1 \times 10^{17} \mathrm{H} / \mathrm{cm}^{2}$. At an implantation dose of $7 \times 10^{16} \mathrm{H} / \mathrm{cm}^{2}$ cleavage took place in part at the same depth, but also short of the peak in the hydrogen concentration at a depth collocated with the maximum in the damage distribution (see figure 5-35, p. 105). In the sample studied here, the ion irradiation induced amount of damage measured by ion channeling is comparable to that produced in the sample implanted with $42 \mathrm{keV} \mathrm{H}$-ions at cryogenic temperatures to a dose of $7 \times 10^{16} \mathrm{H} / \mathrm{cm}^{2}$, where damage accumulation was high enough to cause an increase in the fracture toughness of the material. That suggests that the silicon crystal here is apparently damaged enough to increase the fracture toughness of the material in the region of maximum damage. However, the $\mathrm{H}$-ion implantation dose here is higher, thus more hydrogen is present at shallower depths compared to the $7 \times 10^{16} \mathrm{H} / \mathrm{cm}^{2}$ implanted sample (see figure 5-35, p. 105). Therefore, in the sample studied here, at shallower depths the fracture toughness is lower than at the damage peak, the elastic out-of-plane 
strain is high, and, contrary to the sample implanted to $7 \times 10^{16} \mathrm{H} / \mathrm{cm}^{2}$, the $\mathrm{H}$ concentration sufficient to promote (100) H-platelet formation. Consequently, the surface near region of maximum H-platelet density forms somewhat shallower than the region of maximum damage. The surface far region of maximum H-platelet density is located slightly deeper in the sample than the H-concentration peak. This is in accordance with the deeper micro crack location in the samples studied in chapter 5.2 (see figure 5-32, p. 103).

Summarizing above results, the cleavage of the silicon wafer takes place in the surface far region of the H-implantation zone. Consequently, when implanted to $1 \times 10^{17} \mathrm{H} / \mathrm{cm}^{2}$, the implantation temperature and the method of ion-cutting in $\mathrm{H}$-implanted silicon apparently does not influence the location of the Ion-Cut relative to the implantation damage and hydrogen depth distribution. This strengthens the hypotheses made in chapter 5.2 about the mechanisms of the Ion-Cut in high $\mathrm{H}$-dose implanted silicon. The increase of the silicon fracture toughness with increasing lattice damage in combination with crack tip shielding from growing $\mathrm{H}_{2}$-gas bubbles and an high hydrogen concentration at the periphery of the implantation zone makes the end of tail region in the implantation zone the most favorable location for the Ion-Cut. 


\section{Formation Kinetics of Hydrogen Complexes in Silicon and their Influence on the Crystal Damage}

After establishing the physical mechanisms behind the Ion-Cut process this chapter investigates the evolution of the H-concentration and the crystal damage depth profile of $\mathrm{H}$-implanted silicon samples upon thermal annealing at different temperatures. The information is obtained by ERD analysis, SIMS, and RBS channeling. The aim of this study is to elucidate the kinetics of damage accumulation caused by a rearrangement of the implanted hydrogen and to correlate the gained knowledge to the H-platelet depth distributions examined by XTEM analysis. Also explored is the influence, the exfoliation process during surface blistering has on the damage in the silicon crystal using CSTIM analysis.

The substrates in this work were $\langle 100\rangle$ oriented silicon wafers. To investigate the influence of the dopant type on the kinetics of H-rearrangement, the evolution of the crystal damage and the formation of $\mathrm{H}$-platelets upon annealing, the $\mathrm{H}$-ion implantation and the subsequent heat treatments were performed on n-type and p-type silicon, respectively. The resistivity in both substrates was $1-10 \Omega \mathrm{cm}$. The p-type silicon wafer was doped with boron and the resistivity corresponds to a dopant concentration of $1.4 \times 10^{15}-1.5 \times 10^{16} \mathrm{~cm}^{-3}$. The n-type silicon wafer was phosphorous doped and the resistivity corresponds to a dopant concentration of $4.5 \times 10^{14}-4.5 \times 10^{15} \mathrm{~cm}^{-3}$.

The silicon substrates were implanted with $42 \mathrm{keV} \mathrm{H}$-ions to a dose of $2 \times 10^{16} \mathrm{~cm}^{-2}$. To minimize channeling effects, the incident ion beam was oriented at 7 degrees from the surface normal. The ion-implantation was carried out at $77 \mathrm{~K}$ to lessen recovery of the ion irradiation induced damage. The samples were implanted simultaneously, so ion beam heating effects, sample cooling, and implantation energy where identical for all samples. After the ion-implantation one sample of each conductivity type was either left in its as-implanted state or underwent annealing in vacuum for 30 minutes at temperatures of $200{ }^{\circ} \mathrm{C}, 230{ }^{\circ} \mathrm{C}, 260{ }^{\circ} \mathrm{C}, 290{ }^{\circ} \mathrm{C}, 320^{\circ} \mathrm{C}$, $350{ }^{\circ} \mathrm{C}, 380{ }^{\circ} \mathrm{C}$, and $410^{\circ} \mathrm{C}$, respectively. 


\subsection{Evolution of the Hydrogen and Implantation Damage Depth Distribution upon Thermal Annealing}

To examine the evolution of the H-depth distribution upon annealing, ERD and SIMS measurements were performed on all samples. As an example for the ERD results, figure 6-1 plots the spectra obtained from the n-type silicon samples, which were either left in the as-implanted state or underwent annealing at $290{ }^{\circ} \mathrm{C}$ and $410{ }^{\circ} \mathrm{C}$ for 30 minutes, respectively.
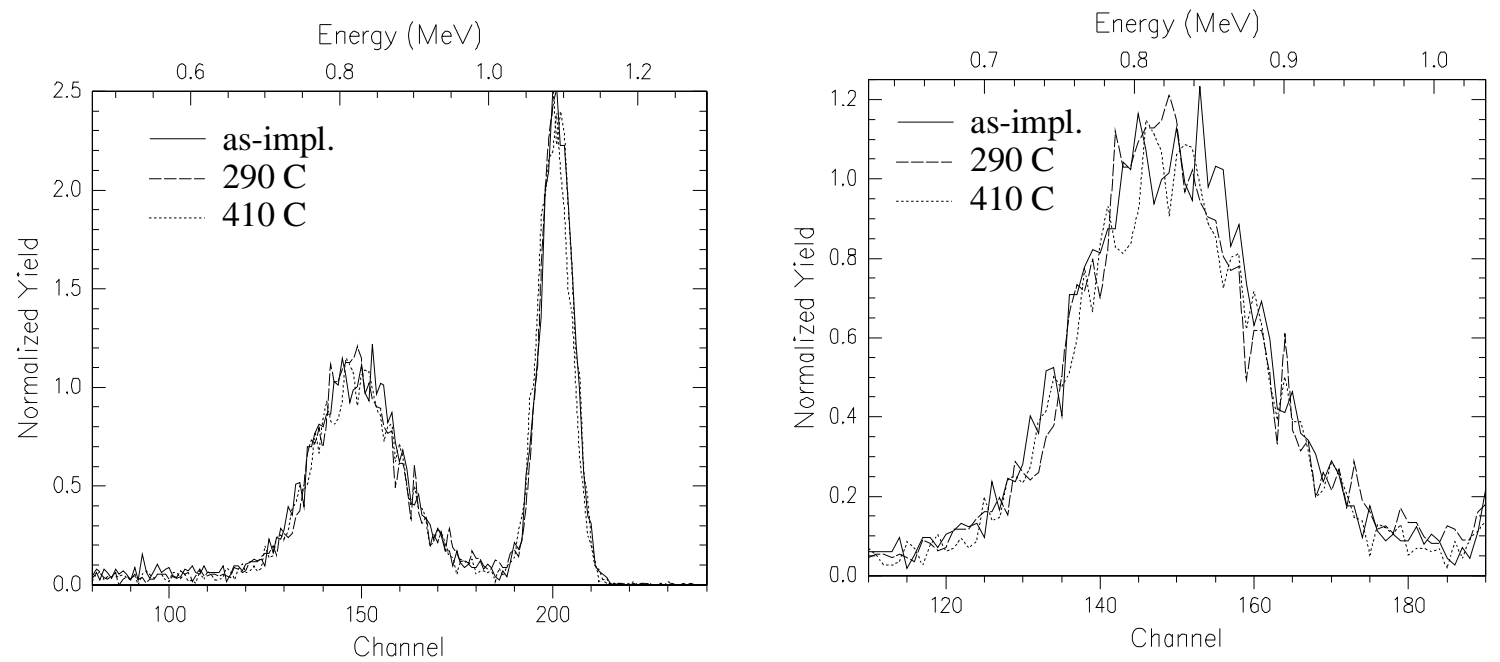

Figure 6-1: $3.0 \mathrm{MeV}$ ERD spectra of the n-type silicon samples, implanted with $42 \mathrm{keV} \mathrm{H}$-ions to a dose of $2 \times 10^{16} \mathrm{~cm}^{-2}$. The sample underwent thermal annealing in vacuum for 30 minutes at different temperatures. The right part of the figure shows only the energy range of the signal, originating from the H-implant.

The signal between channel 115 and 185 is from the hydrogen implant, whereas the right peak centered around channel 200 originates from surface contaminations. The ERD spectra do change only very slightly upon annealing. The enlarged energy slice in the right part of figure 6-1 shows the signal derived from the implanted hydrogen. The figure reveals, that the spectra of the two annealed samples are practically similar. Differences appear to be due to statistical effects. However, the spectrum from the sample in the asimplanted state shows a somewhat broader peak than the ones from the annealed samples.

To derive an accurate H-concentration depth distribution from the ERD spectra, the data were analyzed as described in chapter 4.1.2. The result of the analysis is presented in figure 6-2 for the ERD spectra obtained from the n-type sample in the 
as-implanted state of after annealing at $290^{\circ} \mathrm{C}$ for 30 minutes. The $\mathrm{H}$-concentration is measured to peak at a depth of $442 \mathrm{~nm}$. It appears that the depth region of maximum $\mathrm{H}-$ concentration became somewhat narrower during annealing.

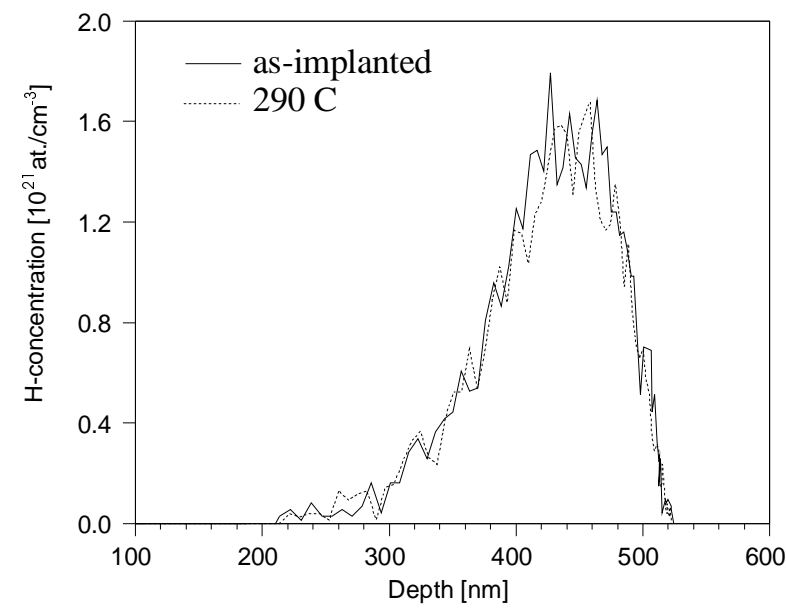

Figure 6-2: H-concentration depth profiles of the n-type silicon sample in the as-implanted sate and after annealing at $290{ }^{\circ} \mathrm{C}$ for minutes. The data were deduced from the ERD spectra from figure 6-1.

Integration of the H-concentration over the whole depth region where the H-implant resides showed that the $\mathrm{H}$-dose in the sample decreases upon annealing to $91.5 \%$ of the $\mathrm{H}$-dose in the as-implanted sample, thus revealing that annealing leads to diffusion of the implanted hydrogen towards the surface or the bulk.

The ERD measurements provide a very precise assessment of the amount of implanted hydrogen remaining in the sample and the depth of the H-concentration peak. However, the energy straggling of the analyzing beam and the forward scattered H-atoms prevent to determine accurately the shape of the H-depth profiles. It is unclear whether the narrowing of the $\mathrm{H}$-concentration peak, observed in figure 6-2, represents the real shape of the H-distribution or is due to low statistics in the measurements. Therefore, in addition to ERD analysis, SIMS was applied to determine the evolution of the H-depth profiles upon annealing. Although SIMS does not provide precise measurement of the depth of the H-concentration profiles and the amount of hydrogen remaining in the samples, it enables an assessment of the shape of the H-concentration depth profiles to a precision not achievable by ERD analysis. Best results are therefore achieved by a combination of both analysis methods: The signal heights in the SIMS spectra are adjusted so that they correspond to the total hydrogen amount in the sample determined by ERD. The time scales in the SIMS spectra were converted into depth scales in a way, that the depths of the H-concentration peaks equal the ones obtained by ERD. Figure 6-3 shows the adjusted SIMS spectra obtained from all the n-type silicon samples. 


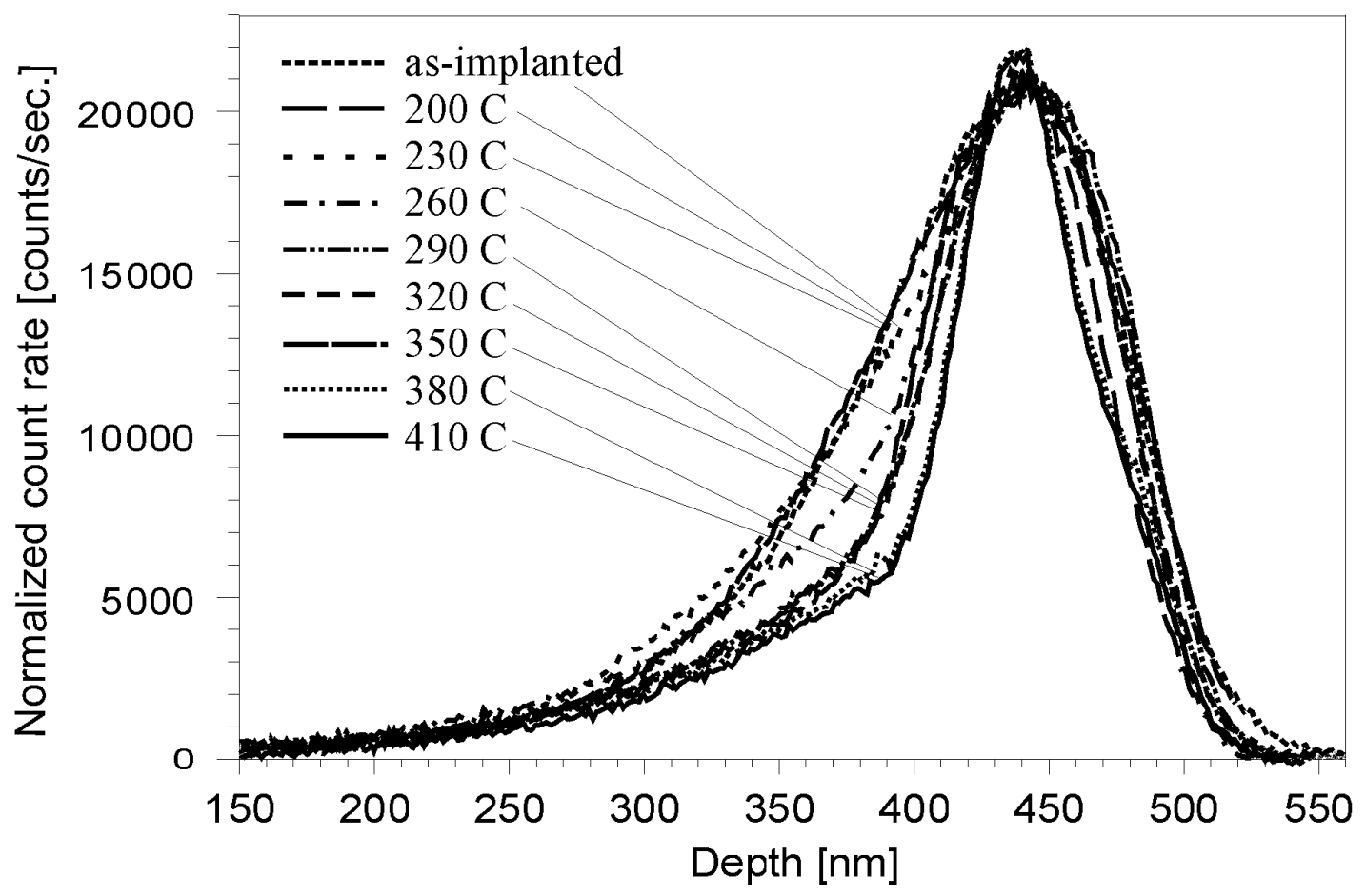

Figure 6-3: SIMS spectra of the n-type silicon samples, implanted with $42 \mathrm{keV} \mathrm{H}$-ions to a dose of $2 \times 10^{16} \mathrm{~cm}^{-2}$, in either the as-implanted state or after annealing for 30 minutes at different temperatures. The spectra were adjusted so that the relative amount of counts corresponds to the by ERD determined amount of hydrogen remaining in the samples and such that the H-concentration peak depth equals the one determined by ERD.

The figure shows a change in the shape of the H-depth profiles upon annealing. In the depth region of maximum hydrogen content, the H-concentration remains unchanged, whereas some out-diffusion occurs for hydrogen, which is located at shallower depths. The data show furthermore, that diffusion of the implanted hydrogen towards the bulk does not take place in the samples, annealed to temperature below $350{ }^{\circ} \mathrm{C}$. When annealed at $380{ }^{\circ} \mathrm{C}$ or $410{ }^{\circ} \mathrm{C}$, respectively, some narrowing of the H-concentration peak from the deep side is visible. It is not clear however, whether hydrogen diffused towards the bulk or accumulated near the H-concentration peak.

Examining the peak count rate, which corresponds to the peak H-concentration, it becomes evident, that the $\mathrm{H}$-concentration at the depth region of maximum hydrogen remains unchanged and even increases when annealed at $380{ }^{\circ} \mathrm{C}$ and $410{ }^{\circ} \mathrm{C}$, respectively.

A more detailed study of the SIMS spectra shows, that the out diffusion of the implanted hydrogen does not increase continuously with increasing annealing temperature. The data reveal that the H-concentration depth profile remains unchanged when annealed at a temperature of $230{ }^{\circ} \mathrm{C}$ or lower. At an anneal temperature of $260{ }^{\circ} \mathrm{C}$ 
out diffusion of hydrogen in the shallow part of the implantation zone starts and intensifies when the anneal temperature is increased to $290{ }^{\circ} \mathrm{C}$. However, the Hconcentration depth profile remains unchanged in the anneal temperature range of $290{ }^{\circ} \mathrm{C}$ to $350{ }^{\circ} \mathrm{C}$. When the annealing temperature is increased to $380{ }^{\circ} \mathrm{C}$, the $\mathrm{H}$-depth profiles narrows on both sides of the $\mathrm{H}$-concentration and remains unchanged when the anneal temperature is increased to $410{ }^{\circ} \mathrm{C}$.

To investigate how the silicon conductivity type influences the H-diffusion behavior, SIMS spectra were also obtained on all p-type silicon samples. The depth of maximum $\mathrm{H}$-concentration and the integrated counts were again adjusted with the values of the $\mathrm{H}$ concentration peak depth and the $\mathrm{H}$-amount determined from the corresponding ERD measurements. Figure 6-4 presents the results.

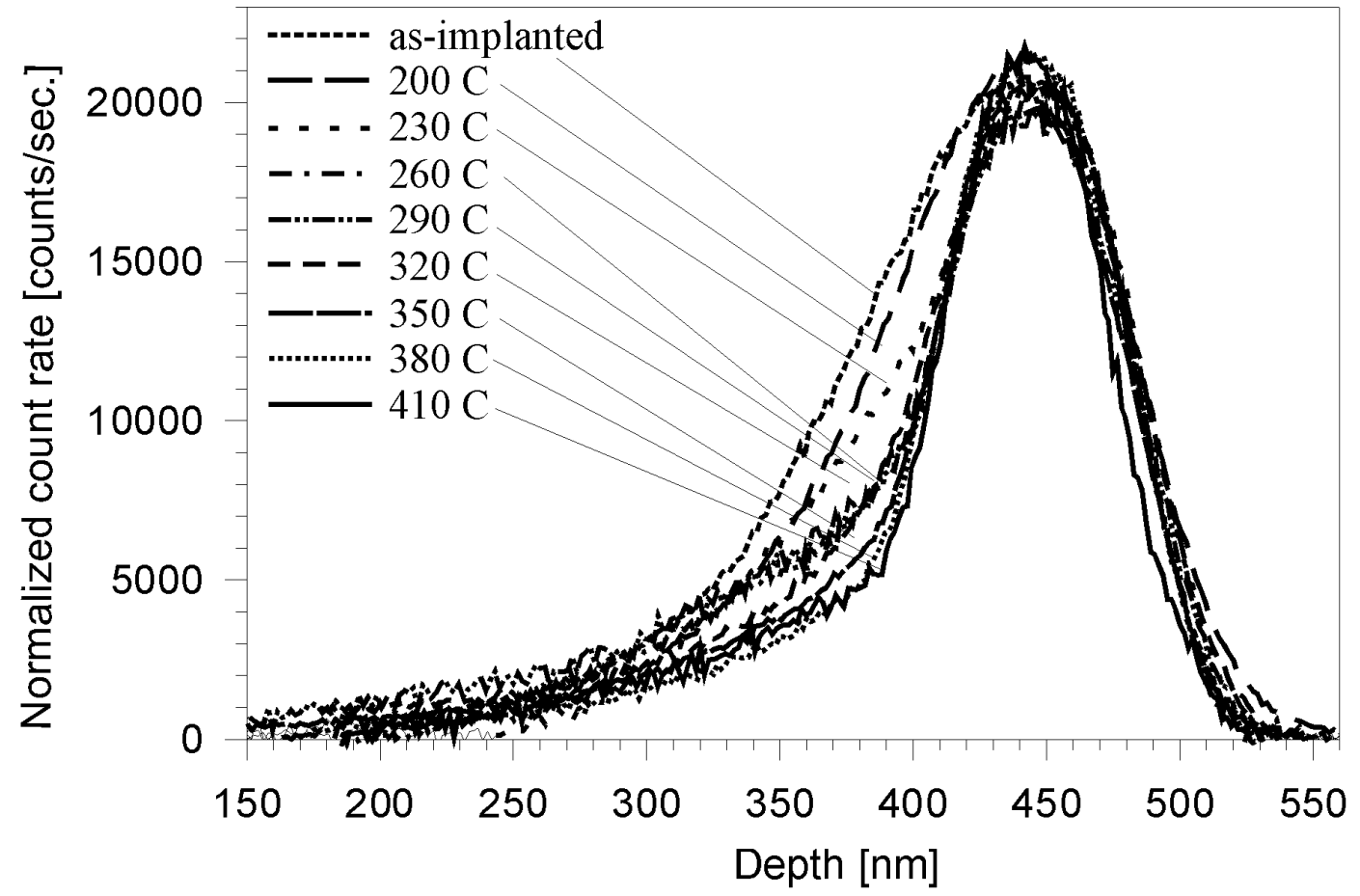

Figure 6-4: Adjusted SIMS spectra of the p-type silicon samples, implanted with $42 \mathrm{keV} \mathrm{H-ions} \mathrm{to} \mathrm{a} \mathrm{dose}$ of $2 \times 10^{16} \mathrm{~cm}^{-2}$, in either the as-implanted state or after annealing for 30 minutes at different temperatures.

The spectra show similar hydrogen diffusion behavior. Hydrogen appears to be trapped in the region of maximum $\mathrm{H}$-concentration and shows some out-diffusion from shallower depths. However, the background dopant type appears to have some influence on the $\mathrm{H}$ depth profile. Comparing these SIMS spectra with the ones obtained from n-type silicon samples (see figure 6-3), it becomes apparent that out diffusion of shallow hydrogen occurs already at an anneal temperature of $200{ }^{\circ} \mathrm{C}$ compared to $260{ }^{\circ} \mathrm{C}$ in the n-type 
silicon samples. Furthermore, the hydrogen out-diffusion seems to be more intense at anneal temperatures of $260{ }^{\circ} \mathrm{C}$ for the p-type sample compared to the n-type sample: The shape of the $\mathrm{H}$-depth profile in the p-type sample after annealing at $260{ }^{\circ} \mathrm{C}$ equals that of the n-type sample, annealed at $290{ }^{\circ} \mathrm{C}$. Finally, the data show that despite the more intense $\mathrm{H}$ out-diffusion in the p-type samples at lower anneal temperatures compared to the n-type samples, the depth region, in which the H-concentration has its maximum and remains almost unchanged upon annealing is broader in the p-type silicon samples. No H-diffusion towards the bulk is observed.

To interpret the changes in the shape of the H-depth profiles it is necessary to obtain the corresponding damage depth profiles. Therefore, RBS channeling measurements were obtained on all samples. Figure 6-5 shows the RBS channeling spectra of every n-type and p-type silicon sample.

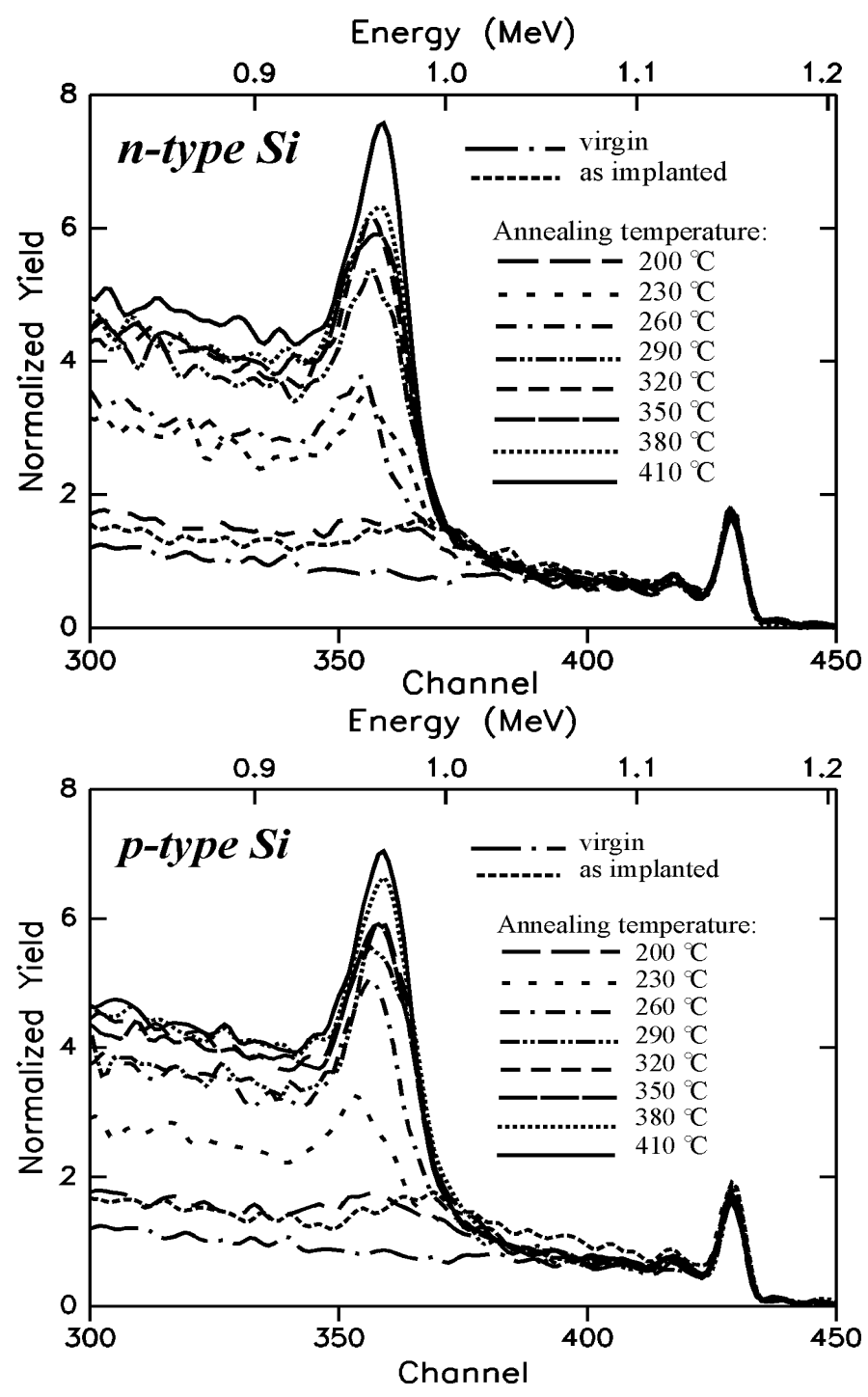

Figure 6-5: $2.0 \mathrm{MeV}$ He RBSchanneling spectra of the $42 \mathrm{keV}$ $\mathrm{H}$-ion implanted $\langle 100\rangle$ n-type and p-type silicon samples, respectively. The data were obtained from the samples in the as-implanted state and after annealing for 30 minutes at different temperatures. The implantation dose was $2 \times 10^{16} \mathrm{H} / \mathrm{cm}^{2}$, the implantation temperature $77 \mathrm{~K}$. The analyzing beam was aligned along the $<100>$ axial direction. 
The data show, that the direct backscattering yield increases considerably with increasing annealing temperature. The maximum channeling yield $\chi_{\max }$ of the as-implanted sample is with $10 \%$ fairly low due to the low $\mathrm{H}$-implantation dose. However, after annealing, $\chi_{\max }$ increases up to a value of about $60 \%$. The channeling data reveal furthermore an increase of the dechanneling yield (channel 300-340) upon annealing. The dechanneling yield does not grow continuously with raising annealing temperature. Its growth rather takes place in two steps, the first occurring at an anneal temperature of $230{ }^{\circ} \mathrm{C}$ for both conductivity types, the second at a anneal temperature of $290{ }^{\circ} \mathrm{C}$ for the n-type silicon samples and at $260{ }^{\circ} \mathrm{C}$ for the p-type silicon samples. This is unexpected behavior in ion-implanted silicon. In general, annealing causes recovery of ion irradiation induced lattice damage and consequently a decrease of the crystal damage.

To gain more detailed information about the damage depth distribution, the channeling data were analyzed as described in chapter 4.2.1. Figure 6-6 shows the obtained results. The figure plots the density of displaced silicon atoms as a function of depth for both, the n-type and the p-type silicon samples, after annealing at different temperatures.
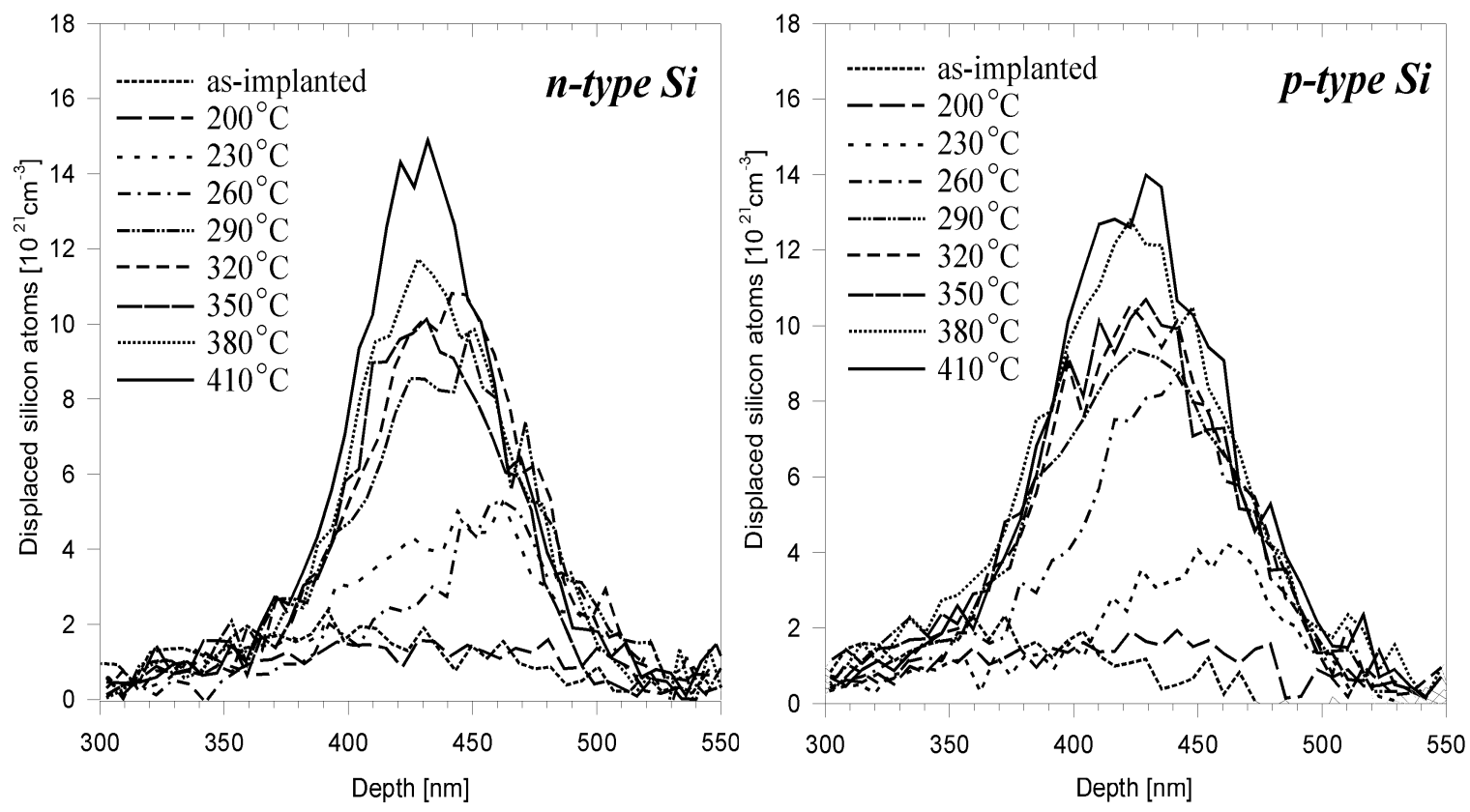

Figure 6-6: Density of displaced silicon atoms in the n-type and p-type silicon samples after $42 \mathrm{keV} \mathrm{H}$ implantation at $77 \mathrm{~K}$ to a dose of $2 \times 10^{16} \mathrm{H} / \mathrm{cm}^{2}$ and vacuum annealing for 30 minutes at different temperatures. The data were deduced from the RBS channeling spectra shown in figure 6-5. 
The damage peak in the as-implanted samples was measured to be $405 \mathrm{~nm}$ deep, independent on the conductivity type of the silicon substrate. The considerable increase of the crystal damage upon annealing at temperatures of $230{ }^{\circ} \mathrm{C}$ and $260{ }^{\circ} \mathrm{C}$ is more pronounced in the deeper part of the implantation zone $(440 \mathrm{~nm}-460 \mathrm{~nm})$, where the implantation damage was low prior to annealing. This depth region comprises the depth layer of maximum $\mathrm{H}$-concentration (see figure 6-3 and 6-4), indicating that the formation of hydrogen related complexes in the implantation zone cause the increase of the damage density. Annealing at temperatures of $290{ }^{\circ} \mathrm{C}$ or higher produces further crystal damage growth at a somewhat shallower depth region between $400 \mathrm{~nm}$ and $450 \mathrm{~nm}$. This depth interval comprises the shallower part of the region of high $\mathrm{H}$-concentration (see figures 6-3 and 6-4).

Comparing the damage growth above the anneal temperature of $290{ }^{\circ} \mathrm{C}$ in the n-type samples with the one in the p-type samples, it becomes evident that the damage depth distribution is narrower in the n-type silicon samples. SIMS observations showed earlier that the hydrogen depth distribution after annealing at these temperatures is also narrower in the n-type samples compared to the corresponding p-type samples. This similarity in the hydrogen and damage depth profiles also suggests that the damage growth is correlated with a restructuring of the implanted hydrogen within the depth region of high $\mathrm{H}$-concentration.

To illustrate relevant characteristics in the crystal damage growth kinetics upon annealing, certain features are deduced from figure 6-6. Figure 6-7 plots the amount of damage in each sample, obtained by integrating the damage density over the whole depth range where crystal damage appears.

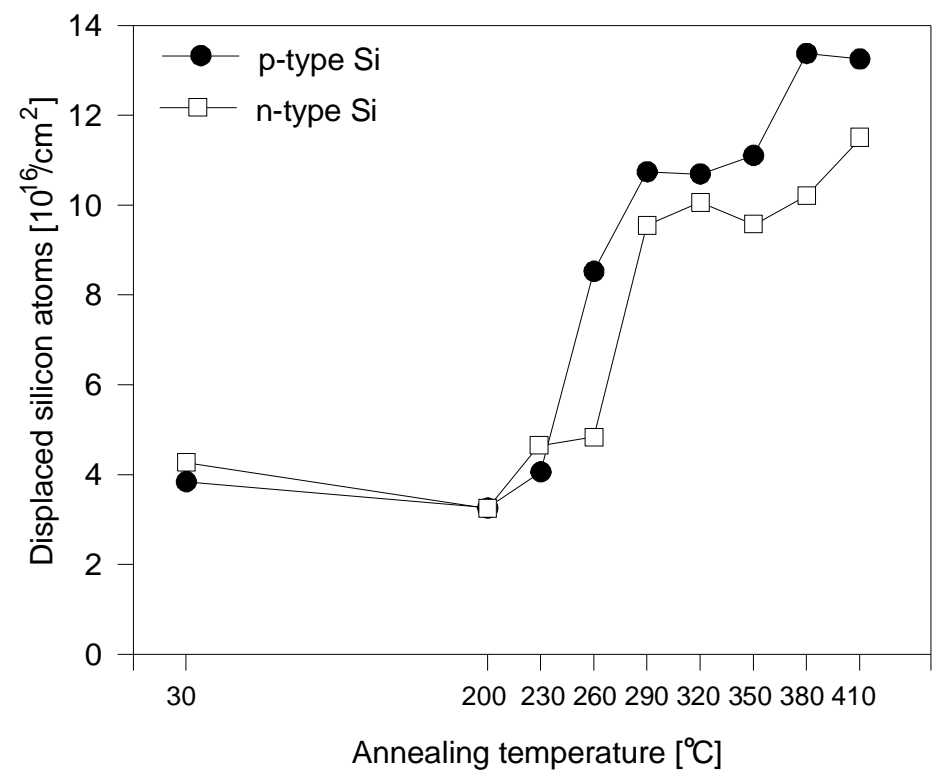

Figure 6-7: Amount of displaced $\mathrm{Si}$ atoms in the implantation zone as a function of annealing temperature. The values are plotted for both silicon conductivity types. The data are deduced from figure 6-6. 
The calculations reveal that the $\mathrm{H}$-ion irradiation caused only $4 \times 10^{16}$ displaced atoms $/ \mathrm{cm}^{2}$ in the as-implanted samples. This corresponds to two displaced silicon lattice atoms per incoming proton. However, TRIM calculations [75] predict 14 displaced silicon atoms per incoming proton. TRIM does not account for damage recovery effects during the ion irradiation process. Therefore, the discrepancy in the experimental and computer simulation result suggests that about $85 \%$ of the ion irradiation induced displacement damage recovers during the $\mathrm{H}$-ion implantation. This is surprising, considering the low implantation temperature of $77 \mathrm{~K}$. However, single vacancies and isolated silicon self-interstitials are known to be very mobile even at low temperatures. $[139,140]$ Their high mobility in combination with the relatively low amount of displaced silicon atoms in the implantation damage zone results in an intrinsic defect evolution, which is largely governed by the recombination of Frenkel pairs and only moderately by the aggregation of vacancies and self-interstitials inside the damaged region. In addition, beam-heating effects lead to a temperature increase in the implantation zone. It should be noted hereby, that beam heating occurs mainly in the depth region, where the kinetic energies of the incoming proton and displaced silicon atoms are too low to cause the further displacement of silicon atoms from their lattice sites. The ion energy is then transferred to the silicon crystal in form of lattice vibrations. This, in turn, leads to a local temperature increase in the depth region of maximum damage and $\mathrm{H}$-concentration and thus has an enhancing effect on the ability of lattice damage to recover. The little amount of crystal damage can be further explained by the low H-implantation dose: The interaction of the implanted $\mathrm{H}$-atoms with lattice defects prevents recombination of Frenkel pairs and consequently a lower H-content has a lower retarding impact on damage recovery.

Annealing at $200{ }^{\circ} \mathrm{C}$ leads to a slight reduction of the crystal damage. A reexamination of the RBS channeling spectra in figure 6-5 suggests that this is due to damage recovery at shallow depths and the very small damage increase in the larger depth region of high $\mathrm{H}$-concentration. Although out-diffusion of shallow hydrogen takes already place in the p-type samples, the hydrogen rearrangement apparently causes only minor silicon atom displacement.

Figure 6-7 shows that an increase of the annealing temperatures above $200{ }^{\circ} \mathrm{C}$ leads to a rapid increase of displaced silicon atoms. On the other hand, the SIMS measurements show only moderate changes in the H-depth profiles. Thus, the combination of crystal damage and $\mathrm{H}$-concentration measurements suggest that thermally induced rearrangement of the implanted hydrogen atoms on a microscopical level lead to the observed increase of the crystal damage. 
Infrared studies of implanted hydrogen at comparable implantation conditions revealed such changes in the hydrogen complex formation. [35,36] Implanted H-atoms form complexes of the form $V_{x} H_{y}$ or $I_{x} H_{y}$ where $V$ denotes a silicon vacancy and $I$ denotes a silicon interstitial, and the subscript have values of $x=1$ and 2 and $y=1-4$. Also observed was the so-called $\mathrm{H}_{2}{ }^{*}$ complex, a hydrogen molecule formation, where one $\mathrm{H}$ atom is located at the bond centered site and the other at the antibond site with a silicon lattice atom residing in between the $\mathrm{H}_{2}$ bond (see right image in figure 2-3, p. 14). This diversity of hydrogen complexes with defects is formed already in the as-implanted state. The stability of these hydrogen defect complexes varies. During annealing, some of them dissociate and convert into others. For example, upon annealing of $\mathrm{H}$-ion implanted samples, the IR studies uncover a net loss of bound hydrogen and agglomeration of hydrogen at existing vacancies.

With further annealing, a net conversion from $\mathrm{Si}-\mathrm{H}$ complexes into $\mathrm{H}_{2}$ at elevated temperatures takes place. $[35,36]$ This process has been found to considerably enlarge the lattice, thus introducing lattice strain into the implantation zone. [136] $\mathrm{The}_{2} \mathrm{H}_{2}$ molecule formation leads to an energy gain that counterbalances the strain build up around the $\mathrm{H}_{2}$ molecules. [42] The strain energy accumulates in the silicon lattice as elastic energy until the number of $\mathrm{H}_{2}$ molecules is high enough to produce a Frenkel pair. [152] This finally leads to the observed rise in the direct backscattering yield in the RBS channeling measurements.

It should be noted that $\mathrm{H}_{2}$ molecules, residing as $\mathrm{H}_{2}$-gas in small sub surface bubbles, were found not to be responsible for the high direct backscattering yields observed in figure 6-5. [42] They induce silicon lattice distortion rather than displacement of silicon lattice atoms from their lattice site, consequently causing mainly a high dechanneling yield.

Thus the observed increase of the lattice damage upon annealing at temperatures above $200{ }^{\circ} \mathrm{C}$ reflects the conversion of the $\mathrm{H}$-implant from various $\mathrm{H}$-complexes into molecular hydrogen residing in the silicon lattice. The higher the anneal temperature is the more hydrogen atoms form $\mathrm{H}_{2}$ molecules, which reside in the silicon lattice.

Figure 6-7 shows that the p-type silicon samples accumulated more crystal damage upon annealing than the n-type silicon samples. This implies, that in p-type silicon more $\mathrm{H}$ atoms formed $\mathrm{H}_{2}$ molecules. Since $\mathrm{H}_{2}$ molecules are the last product of a series of H-complex conversions, I suggest that the conductivity type of the silicon substrate influences the stability of various H-complexes with lattice defects. This would lead to different kinetics in the H-Si complex evolution upon annealing and in turn to a different concentration of $\mathrm{H}_{2}$ molecules in p-type and n-type silicon samples. 
Previous experiments have shown that the presence of boron reduces the damage accumulation caused by ion implantation in silicon. [153] This was confirmed by own experiments on silicon samples with different dopant types and dopant levels. The silicon substrates were implanted with $42 \mathrm{keV} \mathrm{H}$-ions to a higher dose of $5 \times 10^{16} \mathrm{~cm}^{-2}$ to produce more damage in the samples in the as-implanted state. RBS channeling measurements (not shown) revealed a decrease of the implantation damage in the asimplanted samples with increasing boron dopant level. The experiments were also carried out on the silicon substrates, used in this study. RBS channeling analysis indeed showed lower damage accumulation in the p-type silicon sample compared to the n-type silicon sample.

This difference in the damage accumulation during the $\mathrm{H}$-implantation process indeed leads to different H-complex formation kinetics in the n-type and p-type substrates: Since damage in the as-implanted state is lower in the p-type sample, it is expected that upon annealing, in the p-type samples more $\mathrm{H}$-atoms liberate from lattice defects and subsequently form $\mathrm{H}_{2}$ molecules. It should be noted, that the retarding effect, the boron dopants have on the damage accumulation in silicon was observed for implantation species, different from H-ions. [153] Therefore, the difference in the damage yields in the as-implanted n-type and p-type silicon sample are not expected to be caused by different $\mathrm{H}$-complex formations in the two samples.

The transformation of the $\mathrm{H}$-implant into $\mathrm{H}_{2}$ molecules explains also the shape of the hydrogen depth profiles, obtained by SIMS (figure 6-3 and 6-4). In regions of high $\mathrm{H}$-concentration, $\mathrm{H}$-atoms, when detrapped from lattice defects, will come in close proximity of other $\mathrm{H}$-atoms after traveling only short distances through the lattice and form $\mathrm{H}_{2}$ molecules. Therefore, in regions of high $\mathrm{H}$-concentration, the likelihood of molecule formation is very high. $\mathrm{H}_{2}$ molecules in silicon, however, are known to be very immobile. Thus, high H-concentrations lead to self-trapping of hydrogen in silicon.

So far, the combination of RBS channeling, ERD analysis and SIMS made it possible to establish the cause for the increase of the crystal damage with increasing annealing temperatures and the trapping of the implanted hydrogen residing in the region of maximum H-concentration. However, more information about the rearrangement of the implanted hydrogen can be deduced from the RBS channeling data by analyzing the shape and depth of the damage depth profiles. 
To investigate the shift of the damage in more detail, figure 6-8 plots the depth of maximum damage as a function of annealing temperature. The data were deduced from figure 6-6.

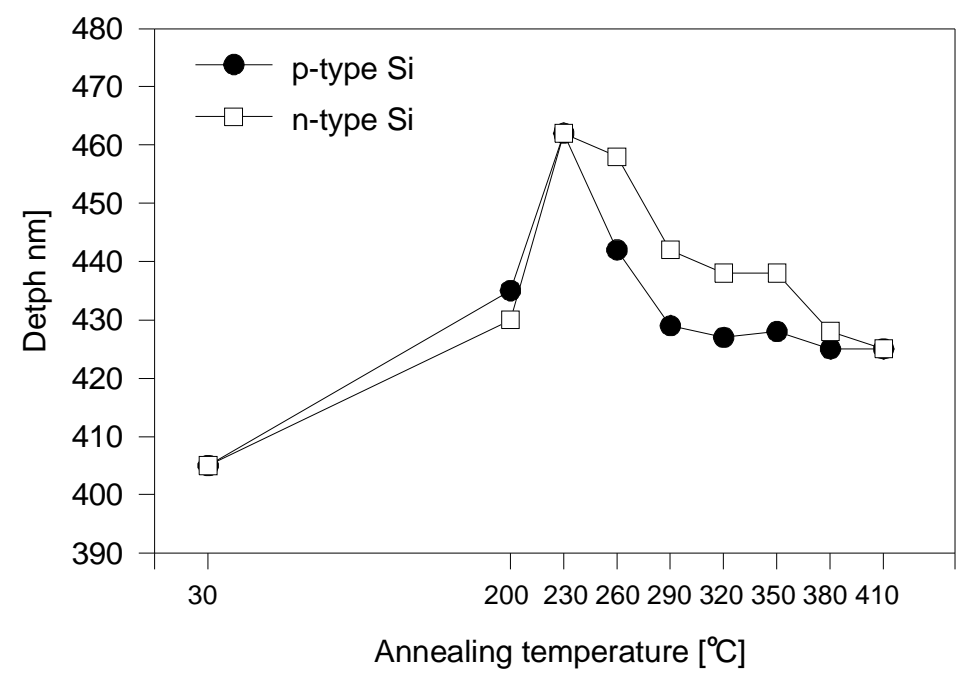

Figure 6-8: Depth of the damage peak as a function of annealing temperature for both silicon conductivity types. The data are deduced from figure 6-6

The data show an increase of the damage peak depth from $405 \mathrm{~nm}$ to $462 \mathrm{~nm}$ upon increasing annealing temperature up to $230{ }^{\circ} \mathrm{C}$. Further increase of the anneal temperature leads to a shift of the damage peak towards a shallower depth of $425 \mathrm{~nm}$. This shift is more rapid for the p-type samples compared to the n-type samples. In the p-type material, the shift of the damage peak is almost completed after annealing at $290{ }^{\circ} \mathrm{C}$, whereas n-type samples require an annealing temperature of $380^{\circ} \mathrm{C}$ to almost reach the final damage peak depth location.

The region of highest crystal damage in the as-implanted samples is centered around a depth shallower than the depth of maximum H-concentration. This depth layer of high damage acts as an effective getter for hydrogen atoms and thus delays the formation of $\mathrm{H}_{2}$ molecular species in the damage layer. On the other hand, the high concentration of atomic hydrogen in the deeper region centered around a depth of $442 \mathrm{~nm}$, leads to a shorter hydrogen capture radius for $\mathrm{H}_{2}$ molecule formation. This explains the shift of the damage peaks upon increasing annealing temperature: Initially, at annealing temperatures of $200{ }^{\circ} \mathrm{C}$ and $230{ }^{\circ} \mathrm{C}$, the temperature is not high enough to cause significant detrapping of hydrogen from lattice defects. $\mathrm{H}_{2}$ molecule formation takes place preferred in the deep part of the implantation zone, where the ion-irradiation induced lattice damage is low, such that $\mathrm{H}$-atoms are not trapped as intensely to various lattice defects as in the shallower depth region of high damage. Consequently, the crystal damage increases initially pronounced at larger depths. In the end of tail region of the $\mathrm{H}$ depth distribution, lattice damage is minimal, but $\mathrm{H}$-concentration high. This explains 
the shift of the damage peak beyond the depth of maximum H-concentration, which was measured to be $442 \mathrm{~nm}$ (see figure 6-2). Increasing the annealing temperature further, detrapping of $\mathrm{H}$-atoms from lattice defects becomes more intense, thus enabling the atomic hydrogen in the region of high as-implanted damage to form $\mathrm{H}_{2}$ molecules. $\mathrm{H}_{2}$ molecule formation in the deeper region with highest $\mathrm{H}$-concentration is already completed and therefore an increase of the annealing temperature does not lead to a further increase of displaced silicon atoms in that depth layer. Consequently, the damage peak shifts towards the shallower depth of high as-implanted damage.

Interestingly, this process takes place at lower temperatures for the p-type samples compared to the n-type samples. Comparing this observation with the out-diffusion behavior of hydrogen located in the shallower region, i.e. the region of highest damage in the as-implanted samples (see figure 6-3 and 6-4), it becomes evident that these two findings are correlated: In p-type samples, the lattice damage recovers faster upon annealing and hence atomic hydrogen is less strongly tapped at lattice defects compared to the n-type samples. Thus, annealing at temperatures of $260{ }^{\circ} \mathrm{C}$ or higher enables the liberation of more $\mathrm{H}$-atoms within the damage layer in the p-type silicon compared to n-type silicon. Consequently, $\mathrm{H}$ out-diffusion and $\mathrm{H}_{2}$ molecule formation in the damage layer are more pronounced in the p-type material. This is in accordance with the SIMS and RBS channeling observations. SIMS measurements revealed that out-diffusion of hydrogen located in the damage layer is more pronounced in p-type material at annealing temperatures between $200{ }^{\circ} \mathrm{C}$ and $290{ }^{\circ} \mathrm{C}$, indicating a larger amount of free hydrogen in the damage layer (see figure 6-3 and 6-4). RBS channeling results showed a more rapid shift of the damage peak towards shallower depths upon annealing at temperatures between $200{ }^{\circ} \mathrm{C}$ and $290{ }^{\circ} \mathrm{C}$, indicating a higher $\mathrm{H}_{2}$ molecule density in the damage layer compared to that in n-type silicon. (see figure 6-6)

Recent experimental research, using the combination of spreading resistance probe (SRP) analysis and SIMS, revealed the creation of molecular hydrogen species is silicon at a temperature of about $260^{\circ} \mathrm{C}$. [154] This is in agreement with the findings from the present study. However, the experimental research mentioned above [154] also showed, that these molecular hydrogen species are metastable and decay at $450{ }^{\circ} \mathrm{C}$. In the present study, the maximum annealing temperature was $410{ }^{\circ} \mathrm{C}$ and thus, the $\mathrm{H}_{2}$ molecules in the silicon lattice are expected to be stable in accordance to the obtained results.

To confirm the conclusions, drawn from the observed H-concentrations and damage depth profiles, the anneal temperature was further increased in $30 \mathrm{~K}$ steps up to 
$530^{\circ} \mathrm{C}$. These high temperature annealed samples were also analyzed by RBS channeling, ERD, and SIMS.

Figure 6-9 plots the SIMS spectra, obtained from the n-type samples. The SIMS data were adjusted with the results of the corresponding ERD measurements.

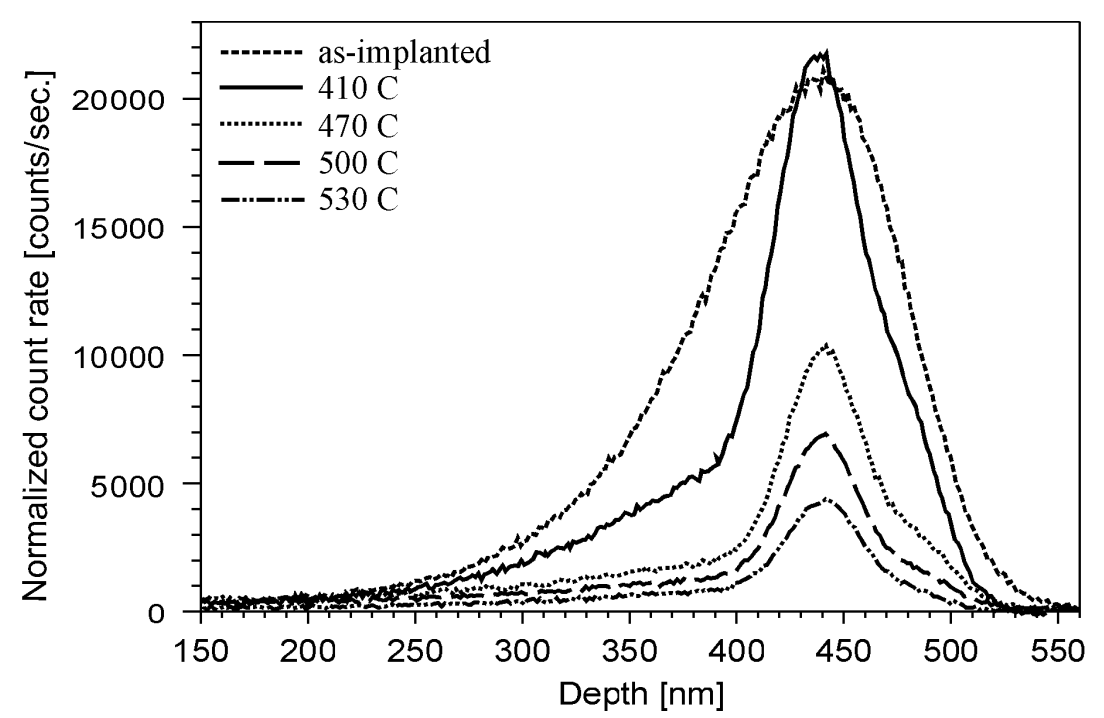

Figure 6-9: SIMS spectra of the n-type silicon samples, implanted with $42 \mathrm{keV} \mathrm{H}$-ions to a dose of $2 \times 10^{16} \mathrm{~cm}^{-2}$, in either the as-implanted state or after annealing for 30 minutes at different temperatures. The spectra were adjusted so that the relative amount of counts corresponds to the by ERD determined amount of hydrogen remaining in the samples and such that the $\mathrm{H}$-concentration peak depth equals the one determined by ERD analysis.

While heat treatment at annealing temperatures up to $410{ }^{\circ} \mathrm{C}$ did not produce significant hydrogen out-diffusion, annealing at higher annealing temperatures leads to a fast decrease of the implanted hydrogen. Hydrogen molecules dissociate [154], producing fast diffusion atomic hydrogen. At annealing temperatures above $450{ }^{\circ} \mathrm{C}$ the $\mathrm{H}$-atoms do not get re-trapped at lattice defects. This results in very mobile H-atoms, which diffuse towards the wafer surface, into the bulk material, or get trapped in H-platelets or bubbles.

Figure 6-10 presents the RBS channeling spectra obtained from the same n-type silicon samples. 


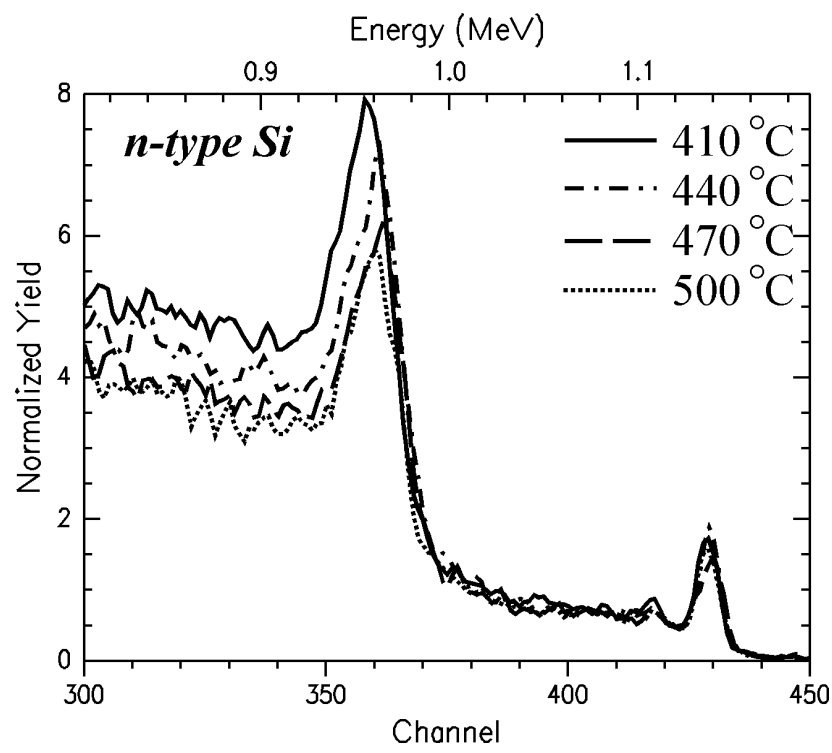

Figure 6-10: $2.0 \mathrm{MeV}$ He RBSchanneling spectra of the $42 \mathrm{keV} \mathrm{H}-$ ion implanted <100> n-type silicon samples after annealing for 30 minutes at different temperatures. The implantation dose was $2 \times 10^{16} \mathrm{H} / \mathrm{cm}^{2}$, the implantation temperature $77 \mathrm{~K}$. The analyzing beam was aligned along the $\langle 100\rangle$ axial direction.

The channeling data reveal that as the hydrogen content declines, the amount of displaced silicon atoms also declines, suggesting that they are strictly correlated. This is in agreement with the proposed influence of the hydrogen molecules on the silicon crystal damage. Unlike the direct backscattering yield, the dechanneling yield (channel 300 - 345) does not decline as fast. High dechanneling originates from a distortion of the silicon lattice. Therefore, I suggest that hydrogen filled platelets or bubbles are still present in the implantation zone and are the main cause for the high dechanneling yield. Indeed earlier infrared vibrational studies have shown, that $\mathrm{Si}-\mathrm{H}$ bonds associated with vacancy and interstitial-related displacement damage are less stable than surface traps of hydrogen at internal platelets [34], in accordance with the suggested presence of $\mathrm{H}$ platelets or bubbles. This hypothesis is confirmed by TEM studies, which showed that the platelet density declines only at anneal temperatures of $600{ }^{\circ} \mathrm{C}$ or above. [42] 


\subsection{Growth Kinetics of Hydrogen Platelets}

So far this chapter described the thermally activated rearrangement of the implanted hydrogen and its effect on the damage accumulation in the implantation zone. However, hydrogen filled platelets or bubbles constitute the final product of this process. Their formation and growth lead ultimately to the exfoliation of the silicon wafer. Thus, it is important to obtain and understand the kinetical evolution of these H-platelets. To examine the H-platelet formation kinetics, XTEM imaging was performed for the sample in the as-implanted state and also after annealing at different temperatures. Figure 6-11 presents, as an example, two low magnification XTEM images from the n-type silicon sample, either in the as-implanted state or after annealing at $260{ }^{\circ} \mathrm{C}$. The images were obtained in an off Bragg condition.
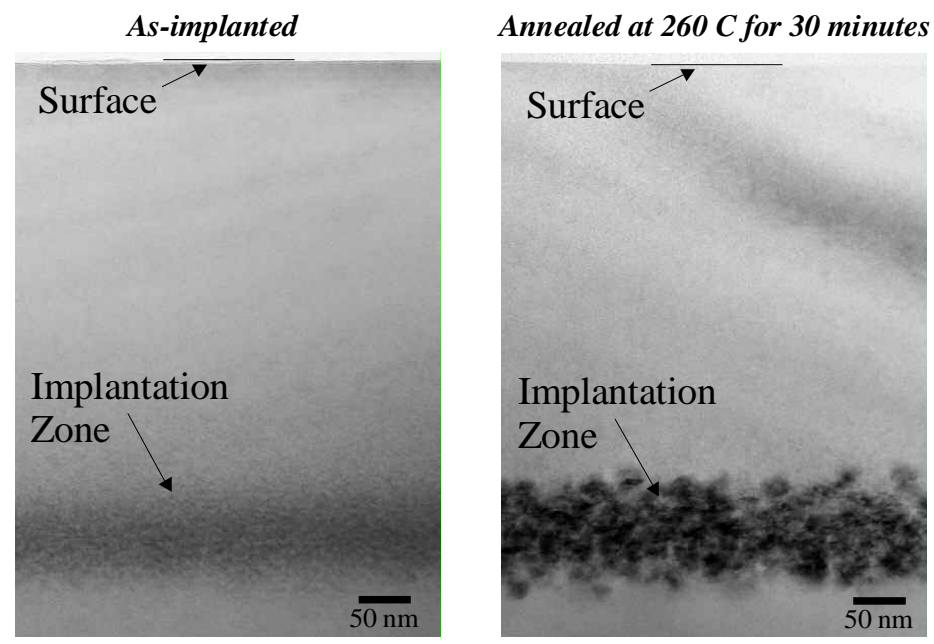

Figure 6-11: Bright field low magnification XTEM image of the n-type silicon sample, viewed edge-on in the [110] Si projection. The sample was implanted at 42 $\mathrm{keV} \mathrm{H}$-with $2 \times 10^{16} \mathrm{H} / \mathrm{cm}^{2}$. The images show the sample in the as-implanted state and after annealing at $260{ }^{\circ} \mathrm{C}$ for 30 minutes

Since the H-ion implantation dose was low, the ion irradiation process did not produce any extended defects or a densely damaged implantation zone. Also, unlike in the sample where $\mathrm{H}$-ions were implanted at room temperature to a dose of $5 \times 10^{16} \mathrm{H} / \mathrm{cm}^{2}$ (see figure $5-10$, p. 75), the formation of H-platelets is not observed here. The slightly damaged zone extends from a depth of about $395 \mathrm{~nm}$ to $490 \mathrm{~nm}$. However, upon annealing at $260{ }^{\circ} \mathrm{C}$ for 30 minutes the implantation zone undergoes a drastic change. The contrast in the implantation zone arises from lattice strain fields of various defects. In particular the XTEM image shows extended defects, which appear to be H-platelets. These platelets show Ashby-Brown contrast [117], corroborating that they act as strain centers. The damaged zone ranges from a depth of $375 \mathrm{~nm}$ to $500 \mathrm{~nm}$. This is somewhat broader than in the as-implanted sample, indicating that the implanted hydrogen rearranges upon annealing causing an increase of the crystal damage. This observation is consistent with 
above RBS analysis, which revealed a considerable increase of displaced silicon atoms during annealing.

To gain more information about the evolution of the implantation zone upon annealing, higher magnification XTEM images were obtained from the n-type samples annealed at different temperatures. Figure 6-12 shows the images of the implantation zones. All XTEM images were obtained also at lower magnification to measure the distance of the platelets to the wafer surface. This enables to deduce the depth distributions of the $\mathrm{H}$ platelet densities. Figure 6-12 also shows the obtained density depth distributions of H-platelets, aligned along the (100) plane parallel to the wafer surface and the H-platelets, lying in the $(1 \overline{1} 1)+(11 \overline{1})$ planes of the silicon crystal. The error in the magnification and consequently in the depth measurements is only about $1 \%$, as determined by TEM standards.

It should be mentioned, that the density of H-platelets aligned parallel to $\{111\}$ planes is higher than the density of $(1 \overline{1} 1)+(11 \overline{1}) \mathrm{H}$-platelets, deduced from our XTEM analysis: The XTEM samples were viewed edge-on in the [110] direction and therefore

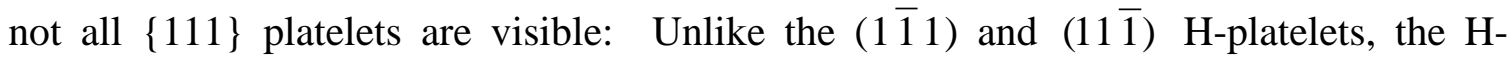
platelets lying in (111) and (111) planes are not viewed edge-on and thus do not appear in the XTEM images. Consequently, we assume the densities of $\{111\}$ H-platelets to be twice as large as the densities of $(1 \overline{1} 1)+(11 \overline{1})$ H-platelets, which are plotted in figure 6-12

The data show, that annealing at $200{ }^{\circ} \mathrm{C}$ leads already to the formation of $\mathrm{H}$-decorated or filled platelets. After annealing at $200{ }^{\circ} \mathrm{C}$ and $230{ }^{\circ} \mathrm{C}$, respectively, the platelets are lying dominantly in $\{111\}$ planes. Platelet formation is confined to the depth region of high hydrogen concentration, as seen by comparing the data with the corresponding ERD or SIMS spectra (figure 6-2 and 6-3). (100) H-platelets form only in the shallow part of the depth region of high $\mathrm{H}$-concentration, whereas $\{111\} \mathrm{H}$-platelets extend over the whole depth layer of high $\mathrm{H}$-concentration. When the annealing temperature is increased to $260{ }^{\circ} \mathrm{C}$, the density of (100) H-platelets increases strongly. They form mainly in a depth interval between $390 \mathrm{~nm}$ to $460 \mathrm{~nm}$. The $\{111\} \mathrm{H}$-platelet density, on the other hand, peaks at a larger depth of $465 \mathrm{~nm}$ and extends to the end of tail region of the $\mathrm{H}$ concentration depth distribution. Upon annealing at higher temperatures, it becomes apparent that the H-platelets in the shallow region, where the crystal damage is higher, are oriented predominately along (100) planes, parallel to the surface. $\{111\}$ H-platelets form mainly at the deep region, but extend nonetheless over the whole implantation zone. 


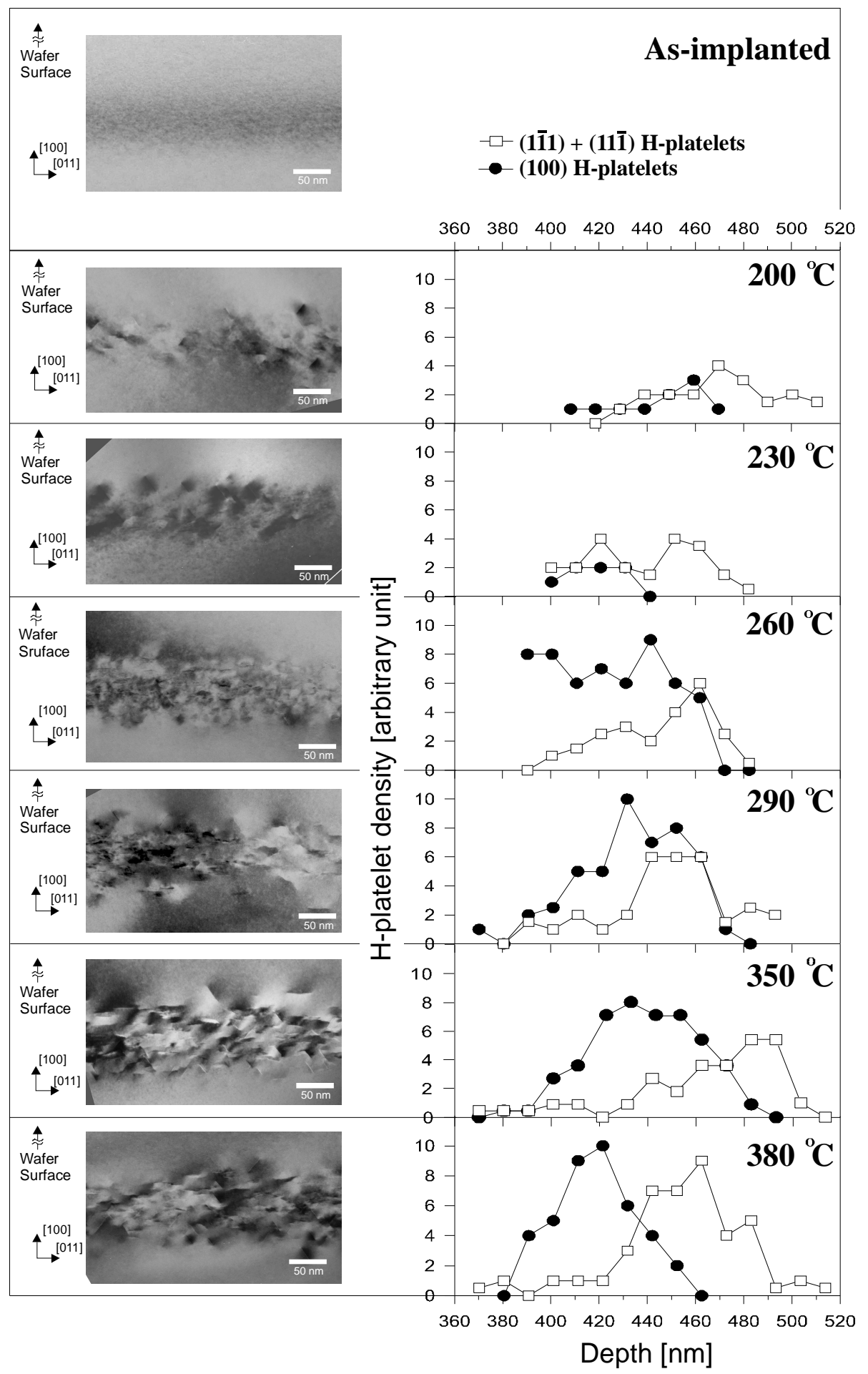

Figure 6-12:

Right part: High magnification bright field XTEM images of the implantation zone in the n-type silicon samples implanted at $42 \mathrm{keV}$ with $2 \times 10^{16} \mathrm{H}$-ions $/ \mathrm{cm}^{2}$. The images were obtained from the sample in the as-implanted state and also after annealing for 30 minutes at different temperatures.

Left part: Density of hydrogen platelets, aligned either parallel to the surface in the (100) plane or lying in the $(1 \overline{\mathbf{1}} 1)+(11 \overline{\mathbf{1}})$ planes, as a function of sample depth. The data were deduced from the XTEM images. 
Comparing the platelet depth distribution after annealing at $350{ }^{\circ} \mathrm{C}$ and $380{ }^{\circ} \mathrm{C}$, the graphs show a narrowing of the (100) H-platelet depth profiles. This indicates a competing behavior in the growth of (100) and $\{111\} \mathrm{H}$-platelets. In the depth region between $430 \mathrm{~nm}$ and $460 \mathrm{~nm}$, the \{111\} H-platelets appear to form and grow on the expense of (100) platelets. This confirms, that H-platelet grow via Ostwald ripening

In all samples, the height of the H-platelets is about $1 \mathrm{~nm}$. However, their diameters vary within one sample and show an increase with increasing temperature. The H-platelet diameters range from $14 \mathrm{~nm}$ to $18 \mathrm{~nm}$ after annealing at $200{ }^{\circ} \mathrm{C}$ and increase to $20 \mathrm{~nm}$ to $36 \mathrm{~nm}$ after annealing at $380{ }^{\circ} \mathrm{C}$. The H-platelets were found to be in average larger at the periphery of the platelet depth distribution than in the center.

The $\{111\}$ planes in the silicon crystal are known to be the energetically most favorable planes for cleavage. [118] A re-examination of the RBS channelling data in figure 6-5 (p. 137) shows that the fairly low annealing temperatures of $200{ }^{\circ} \mathrm{C}$ and $230{ }^{\circ} \mathrm{C}$ do not induce a considerable amount of displaced silicon atoms and strain in the implantation zone. Therefore, in these samples it is most energy efficient to form H-platelets along $\{111\}$ planes.

Unlike for the two lowest annealing temperatures, at $260{ }^{\circ} \mathrm{C}$ and $290{ }^{\circ} \mathrm{C}$, the heat treatment causes a significant rise of the crystal damage and compressive stresses in the implantation zone, which in turn lead to the development of tensile out-of-plane strain (see also chapter 5.1). As described in detail in chapter 5.1, the out-of-plane tensile strain facilitates the incorporation of $\mathrm{H}$-atoms into the bond-centered site between two silicon atoms, thus enhances the formation of (100) H-platelets (see figure 5-11, p. 75). This trend is reflected by the evolution of the H-platelet concentration depth profiles after annealing at these two temperatures: The concentration of $\mathrm{H}$-platelets, aligned along (100) planes, increases drastically and extends over the whole implantation zone. On the other hand, the $\{111\}$ H-platelet concentration increases only moderately. The depth of highest (100) H-platelet concentration equals the depth of highest crystal damage, as can be seen be re-examining figure 6-8. As previously stated, the H-platelets themselves do not cause the crystal damage growth, observed by RBS channeling. It is caused by $\mathrm{H}_{2}$ molecules forming in the silicon lattice. Thus, the data show that the H-platelet and $\mathrm{H}_{2}$-molecule formation coincide. This is expected considering that both the H-platelet formation and the $\mathrm{H}_{2}$ molecule formation require detrapping of the $\mathrm{H}$-implant from various $\mathrm{H}$-defect complexes. 
Annealing at higher temperatures of $350{ }^{\circ} \mathrm{C}$ and $380{ }^{\circ} \mathrm{C}$ results in two distinct depths regions, in which H-platelets, aligned either along (100) or $\{111\}$ planes, form. After annealing at $360{ }^{\circ} \mathrm{C}$, (100) H-platelets center at a depth of $440 \mathrm{~nm}$, whereas the $\{111\} \mathrm{H}$ platelet concentration peaks at a larger depth of $490 \mathrm{~nm}$. After annealing at $380{ }^{\circ} \mathrm{C}$, the (100) H-platelets reach their maximum concentration $420 \mathrm{~nm}$ deep and the $\{111\} \mathrm{H}$ platelets center at a depth of about $460 \mathrm{~nm}$. A comparison with figure 6-8 shows, that at both annealing temperatures, the (100) H-platelet concentration and the crystal damage peak at the same depth. This observation strengthens the proposed influence of crystal damage on the enhancement of (100) oriented H-platelet formation. $\{111\}$-oriented $\mathrm{H}$ platelets form mainly in the deeper region of the implantation zone. There the damage is lower and thus the out-of-plane strain less. Consequently, no facilitating effect on (100) $\mathrm{H}$-platelet nucleation is present and the platelets form along the planes, which are easiest to crack, i. e. the $\{111\}$ planes.

As mentioned above, the $\mathrm{H}$-platelets are larger in diameter at the periphery of the implantation zone. There, the crystal damage is low and therefore the crystals structure not interrupted as frequently as in regions of higher damage. Consequently, the growth of H-platelets along the silicon crystal planes is less disturbed, leading to larger platelet diameters.

To investigate the influence of the silicon conductivity type on the H-platelet depth distributions, XTEM imaging and platelet density analysis was also performed for the ptype silicon samples. Figure 6-13 compares the obtained $(100)$ and $(1 \overline{1} 1)+(11 \overline{1}) \mathrm{H}$ platelet density depth profiles in the p-type silicon samples with the ones in n-type silicon samples after annealing at different temperatures.

Although the silicon substrates are only moderately doped, the conductivity type appears to have some influence on the formation of $\mathrm{H}$-platelets. Comparing the $\mathrm{H}$ platelet distributions after annealing at $230{ }^{\circ} \mathrm{C}$, it becomes apparent, that $\mathrm{H}$-platelet formation is much more pronounced in the p-type sample than in the n-type sample. This again can be correlated with the results of the SIMS and RBS channeling analysis. Unlike in n-type material, the SIMS data showed out-diffusion of hydrogen located in the shallow part of the implantation zone in the p-type material. This indicates, that the heat treatment leads to de-trapping of hydrogen in the shallower part of the implantation zone, which in turn produces mobile hydrogen. Mobile hydrogen atoms are required for the formation of $\mathrm{H}$-platelets. Hence $\mathrm{H}$-platelet formation is more pronounced in the p-type 
silicon sample. The data show that the H-platelet orientation along (100) planes is more pronounced in the p-type silicon sample compared to the corresponding n-type silicon sample.
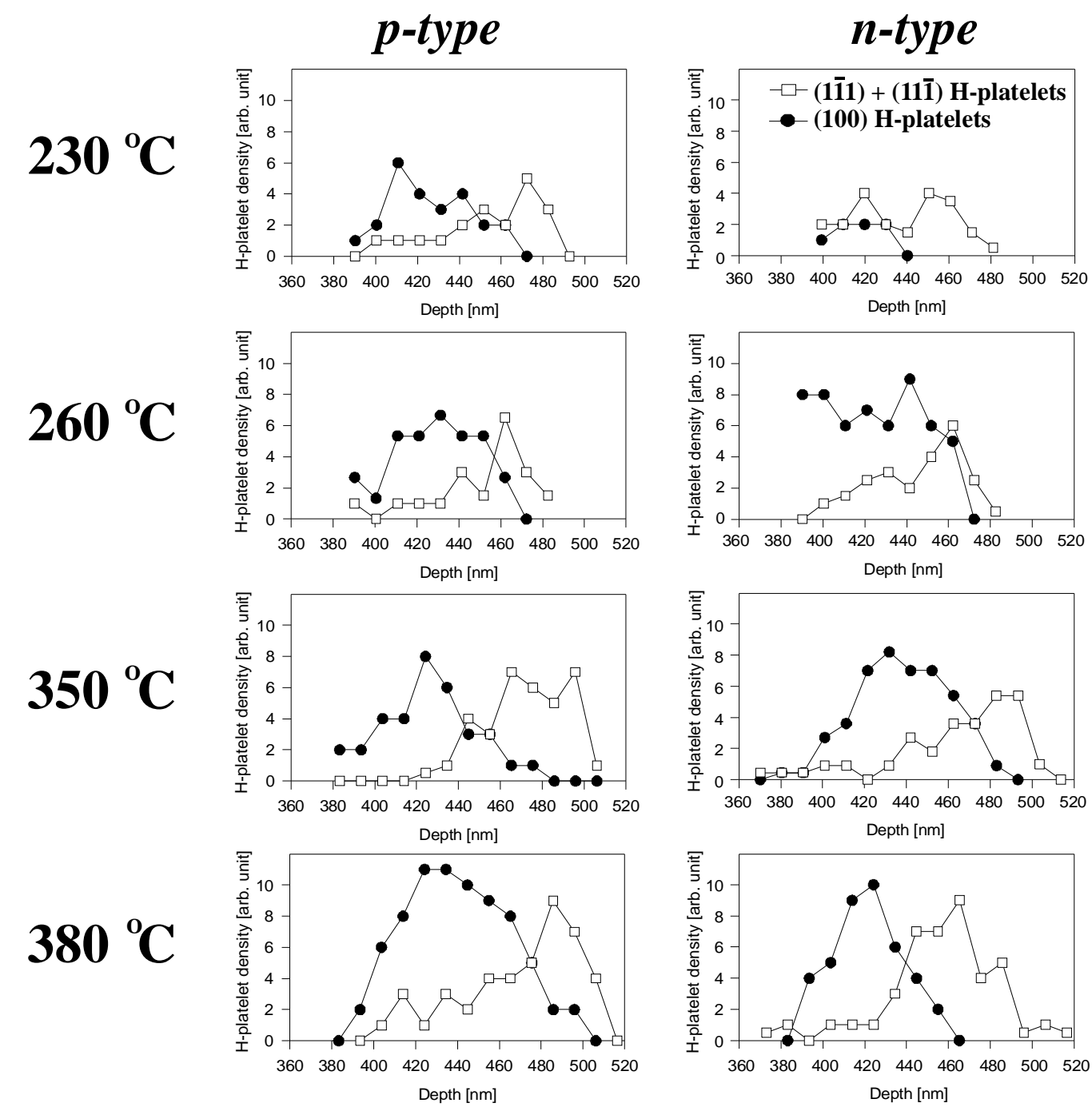

Figure 6-13: Comparison between the H-platelet formation in p-type silicon and the H-platelet formation in n-type silicon for different annealing temperatures. The anneal duration was 30 minutes.

The samples, annealed at $380^{\circ} \mathrm{C}$ show the amount of (100) H-platelets to be roughly twice as high in p-type silicon samples, compared to the corresponding n-type sample. The maximum (100) H-platelet density is higher and the region of high (100) H-platelet concentration extends over a large depth range from about $400 \mathrm{~nm}$ to $480 \mathrm{~nm}$. Due to the enhancing effect of damage on the (100) H-platelet nucleation, the broader damage layer in the p-type sample compared to the n-type sample (see figure 6-6) results in a broader distribution of H-platelets, oriented along (100) planes. 
More information about the evolution of the H-platelet can be deduced from the appearance of Fresnel contrast in the XTEM imaging of H-platelets. As mentioned previously, this phase contrast is due to a change of the electronic potential at the platelets. Such a change in the electronic potential occurs at planar voids or cavities, filled with a very low atomic number element, i. e. hydrogen. Thus, the appearance of Fresnel contrast and its intensity reflects the amount of $\mathrm{H}_{2}$-gas, agglomerated in the $\mathrm{H}$ platelets. The H-platelets in the p-type silicon samples show Fresnel contrast in through focus imaging when annealed at a temperatures of $320^{\circ} \mathrm{C}$ or higher. However, the H-platelets in the n-type samples did show Fresnel contrast only when annealed at $380{ }^{\circ} \mathrm{C}$. Furthermore, after annealing at $380{ }^{\circ} \mathrm{C}$ the observed Fresnel contrast is more intense in the p-type sample than in the n-type sample. These differences in the phase contrast reveal that in p-type samples the transformation of the $\mathrm{H}$-atom decorated platelets into $\mathrm{H}_{2}$-gas filled bubbles is more advanced.

Optical microscopy revealed that upon annealing at $410{ }^{\circ} \mathrm{C}$ the p-type sample formed popped off surface blisters of low density. Such a surface blister formation was not achieved for the n-type samples. However, the final products of the H-complex transformations are $\mathrm{H}_{2}$-gas filled platelets or bubbles, independent of the conductivity type of the silicon substrate. Consequently, upon annealing eventually both substrates reach the same amount of hydrogen in form the $\mathrm{H}_{2}$-gas in the platelets or bubbles. Therefore, I suggest that surface blistering occurs only in p-type material because the different conductivity types show different characteristics in the H-platelet alignments. After annealing at $380{ }^{\circ} \mathrm{C}$ the p-type sample differs from the n-type samples in a way that the p-type sample shows more H-platelets aligned parallel to the wafer surface. Thus, in p-type material, crack propagation through the whole wafer involves a lower number of $\mathrm{Si}-\mathrm{Si}$ bond breaks and consequently requires less energy than in n-type samples, where the amount of H-platelets aligned along (100) equals the amount of H-platelets aligned along $(1 \overline{1} 1)+(11 \overline{1})$ planes. This hypothesis is strengthened by previous studies on the effect the silicon wafer orientation has on surface blister formation kinetics. [155] The study showed, that in $\mathrm{H}$-implanted silicon wafers of different crystal orientation, the surface blister formation kinetics are controlled by the number of Si-Si bonds, that need to be broken to form popped-off surface blisters. 


\subsection{Mechanically Induced Damage during the Exfoliation of Individual Surface Blisters}

So far this study explored the behavior of ion-implanted hydrogen in silicon and its effect on the evolution of the buried damage layer and H-platelet formation. To gain further knowledge about the Ion-Cut process, it is of high interest to examine the effect the surface blister formation, i. e. the crack propagation through the $\mathrm{H}$-implantation zone, has on the crystalline quality of the cleaved sample.

Above RBS channeling measurements allowed to examine the evolution of the buried implantation damage layer upon thermal annealing. However, as soon as surface blisters form, i. e. the silicon layers located above $\mathrm{H}_{2}$-gas filled subsurface bubbles pop off, RBS channeling cannot provide accurate analysis of the damage in the sample any longer. The channeling spectrum is then composed of two signals, one coming from nonblistered surface area and one from the blistered surface area, where the region of high crystal damage, buried before blistering, is exposed to the surface. It is not possible to single out one from the other. Figure 6-14 shows as an example the plane view SEM image and the RBS channeling spectrum of a p-type silicon sample, which showed surface blistering after annealing. The H-implantation parameters were identical to the samples studied above. However, to intensify surface blister formation upon annealing, the implantation dose was increased to $5 \times 10^{16} \mathrm{H} / \mathrm{cm}^{2}$. The sample was thermally annealed at $390{ }^{\circ} \mathrm{C}$ for 10 minutes in vacuum.
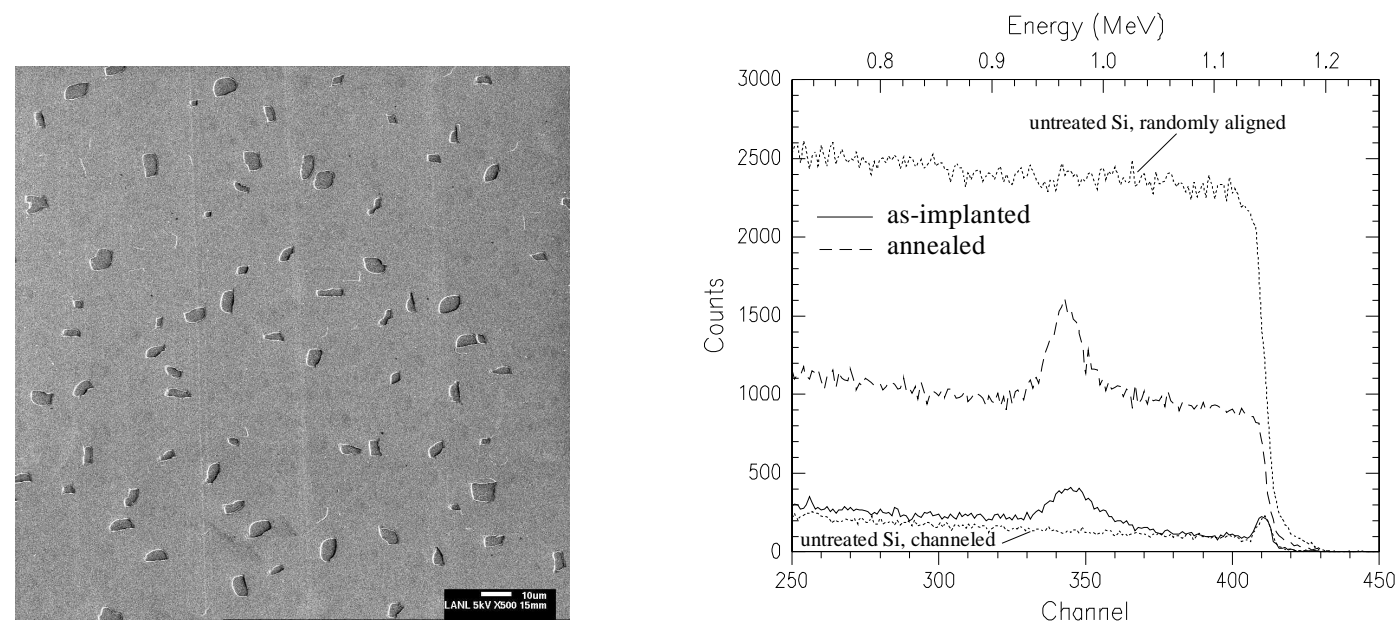

Figure 6-14: Plane view SEM image and 2.0 MeV He RBS-channeling spectrum of the $42 \mathrm{keV} \mathrm{H-ion}$ implanted <100> p-type $\mathrm{Si}$ wafer with a resistivity of $0.005-0.009 \Omega \mathrm{cm}$ after annealing at $390{ }^{\circ} \mathrm{C}$ for 10 minutes in vacuum. The H-implantation dose was $5 \times 10^{16} \mathrm{~cm}^{-2}$. For comparison the RBS channeling spectrum is overlaid with the channeling spectrum of the as-implanted sample and the RBS spectra of untreated silicon at random alignment and in channeling condition. 
The peak in the RBS channeling spectrum from the annealed sample between channel 330 and 360 is due to the buried implantation damage in the non-blistered sample surface area. The spectrum shows a large dechanneling yield at high energies (channel numbers 360-415). The intense dechanneling of the incoming He-ions is due to two characteristics of the sample surface: First, the implantation damage in the blistered sample area is exposed to the surface. Here, the blister bottoms become the depth, where the analyzing beam starts to enter the material and thus induces dechanneling at high energies. Secondly, the deformed silicon layers above $\mathrm{H}_{2}$-gas bubbles, whose pressure was not sufficient to cause the "popping off" of the silicon over layers, lead to additional dechanneling. The SEM image of the blistered sample surface reveals, that only about $6 \%$ of the sample surface is blistered or shows bending due to subsurface hydrogen bubbles. Considering this, it becomes apparent, that the relatively small percentage of blistered and deformed surface area induces a very large dechanneling yield.

The RBS channeling analysis does not allow to separate the dechanneling yield into the fraction caused by the blistered surface areas and the fraction due to the nonblistered but deformed silicon surface areas. Therefore, it is unclear what amount of dechanneling is due to the damage in blistered surface regions. This information would make it possible to examine the effect the exfoliation process has on the crystal damage in the subsurface layer of blister bottoms.

Thus, to gain knowledge about the crystal damage in the blistered sample areas, spatially resolution of the implantation damage measurement is a necessity. The use of unfocused helium beams for channeling analysis does not enable spatially resolved measurements to be made. Therefore, Channeling Scanning Transmission Ion Microscopy (CSTIM (see chapter 4.2.2)) was applied to further examine the effect the exfoliation of individual blisters has on the damage in the sample.

In transmission channeling, channeled ions, which dechannel within the H-ion implanted and annealed sample due to lattice defects or lattice distortion, and ions which are transmitted through the sample in non-channeled alignment lose energy at a high rate. On the other hand, protons, which are transmitted through the sample in channeled trajectories, have a significantly lower electronic stopping power, as described in chapter 4.2. Measurements of the transmitted proton energies at different regions thus give information on variations in crystalline quality within the scanned area.

To facilitate the examination of the damage produced mechanically during the exfoliation process of individual blisters, it is desirable to perform the CSTIM analysis on a sample, which shows relative low damage caused by the ion irradiation process, but nonetheless produces surface blisters upon annealing. Therefore, the analysis was 
performed on the p-type sample with an $\mathrm{H}$-implantation dose of only $2 \times 10^{16} \mathrm{~cm}^{-2}$ which underwent annealing at $410{ }^{\circ} \mathrm{C}$ for 30 minutes. Although the hydrogen implantation dose is very low, this sample produced very view surface blisters during the heat treatment.

Prior to the CSTIM measurements the sample was mechanically thinned and polished to a thickness of $40 \mu \mathrm{m}$. A $2.3 \mathrm{MeV}$ proton beam current of $1 \mathrm{pA}$, focused to an area of $1 \mu \mathrm{m}^{2}$ was then scanned over the sample surface. With transmission channeling, measurements were made of the energies of ions, which are transmitted through the thinned wafers as a function of beam position within the scanned area. Only protons transmitted with an angle of about 0.5 degree with respect to the beam axis were detected. This increases the transmission channeling contrast, since many non-channeled protons are not detected and thus do not contribute to the obtained signal. Images are displayed showing variations in transmitted energy in a position array of $256 \times 256$ pixels.

Figure 6-15 (a) and (b) shows two $200 \times 200 \mu \mathrm{m}^{2}$ transmission channeling images of the same sample area. The sample was tilted in respect to the analyzing beam direction so that channeling in the vertically running [110] planes was obtained (channeling critical angle $\Psi_{p}=0.15^{\circ}$ ). The images show the spatial distribution of counts from different energy slices of the transmitted energy spectra. Figure 6-15 (a) displays counts detected within a lower energy slice of the transmitted energy spectrum than figure 6-15 (b). In all the images, dark areas correspond to a low number of counts, light areas to a higher number of counts.
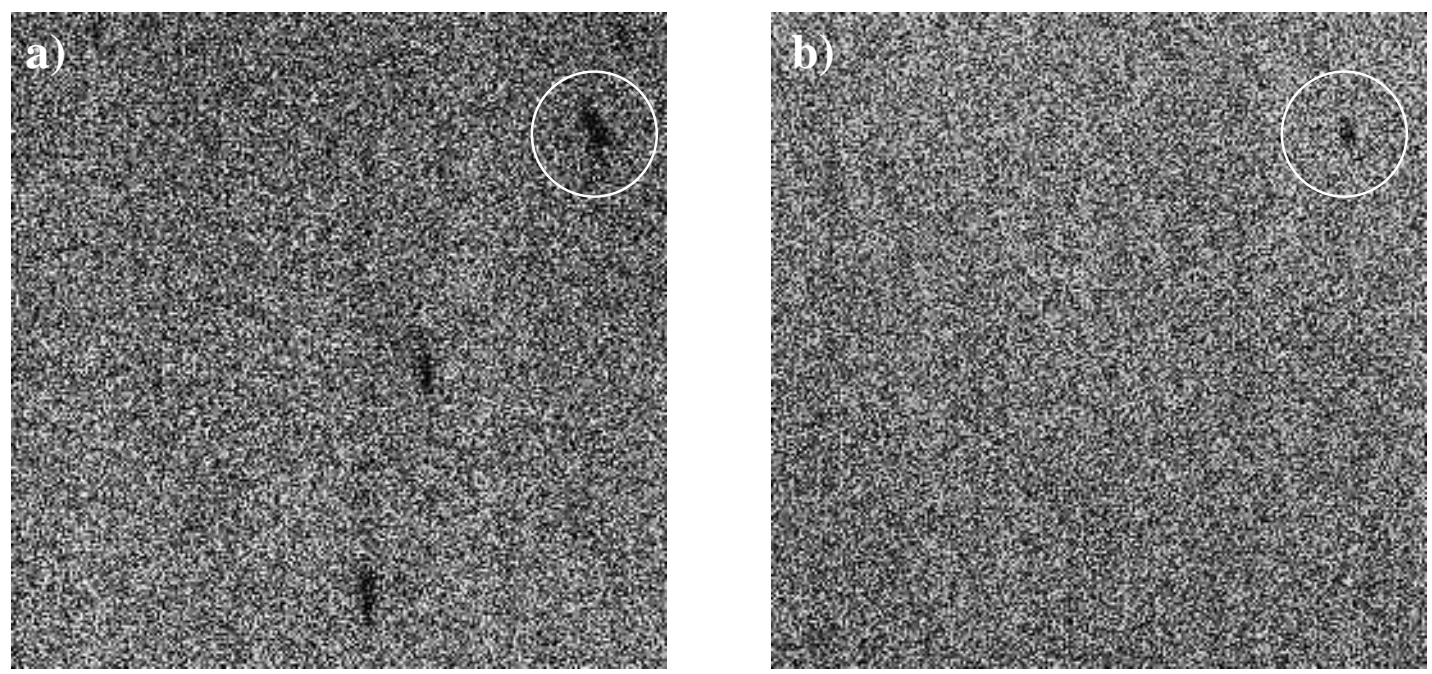

Figure 6-15: $200 \times 200 \mu \mathrm{m}^{2}$ transmission channeling images from the identical sample area of a $\langle 100\rangle \mathrm{p}$ type silicon wafer, implanted with $42 \mathrm{keV}$ H-ions to a dose of $2 \times 10^{16} \mathrm{~cm}^{-2}$ and subsequently annealed at $410{ }^{\circ} \mathrm{C}$ for 30 minutes. The left image (a) was obtained from a lower energy slice of the transmitted energy spectrum than the right image (b). 
Figure 6-15 (a) shows three dark features. These are regions of higher transmitted energy than the surrounding regions. The interpretation of the dark regions is that they are small hydrogen bubbles, which have exfoliated, leaving these regions thinner than the surrounding non-exfoliated material. Interestingly, in figure 6-15 (b), only one such area is still visible (top right, encircled), implying that not all the bubbles have produced the same thickness of peeled off silicon.

To confirm the hypothesis, that these dark areas are surface blisters, higher magnification images were obtained from the dark area, identified by the white circle. Figure 6-16 presents the obtained $25 \times 25 \mu^{2}$ transmission images. The images were obtained either in channeling mode (a) or in random sample alignment, i.e. under nonchanneling conditions (b).
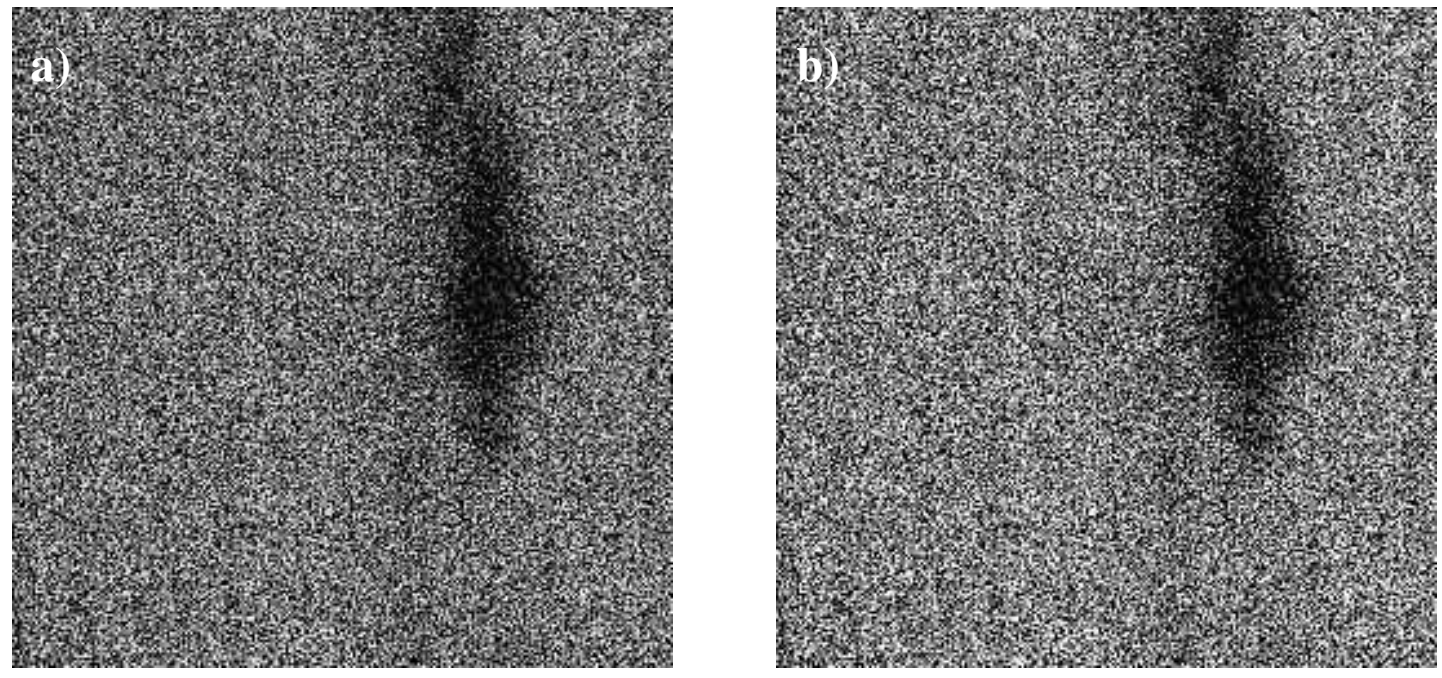

Figure 6-16: $25 \times 25 \mu \mathrm{m}^{2}$ transmission channeling images from the identical sample area of a $\langle 100\rangle \mathrm{p}$ type silicon wafer, implanted with $42 \mathrm{keV}$ H-ions to a dose of $2 \times 10^{16} \mathrm{~cm}^{-2}$ and subsequently annealed at $410^{\circ} \mathrm{C}$ for 30 minutes. The left image (a) was obtained in channeling conditions along the (110) planes, the right image (b) was attained in non-channeling conditions.

The contrast in the channeled and non-channeled transmission images is practically identical, showing that the dark areas indeed represent thinner regions than the surrounding material. Furthermore, the images show more clearly the shape and size of the dark areas. They are asymmetric and their extent is in the order of a few micrometers. Comparing figure 6-16 with surface blisters observed by SEM imaging (see figure 6-14) the shape and size of the dark regions are in accordance with the shape of the surface blisters in the SEM image.

After establishing that the dark features correspond to surface blisters, the transmitted energy spectra associated with the image in figure 6-16 (a) allows the examination of the mechanically induced damage during the exfoliation process of 
individual surface blisters. Figure 6-17 presents the transmission channeling spectra measured at the middle of the exfoliated area (dark region in figure 6-16 (a)) and the surrounding material.

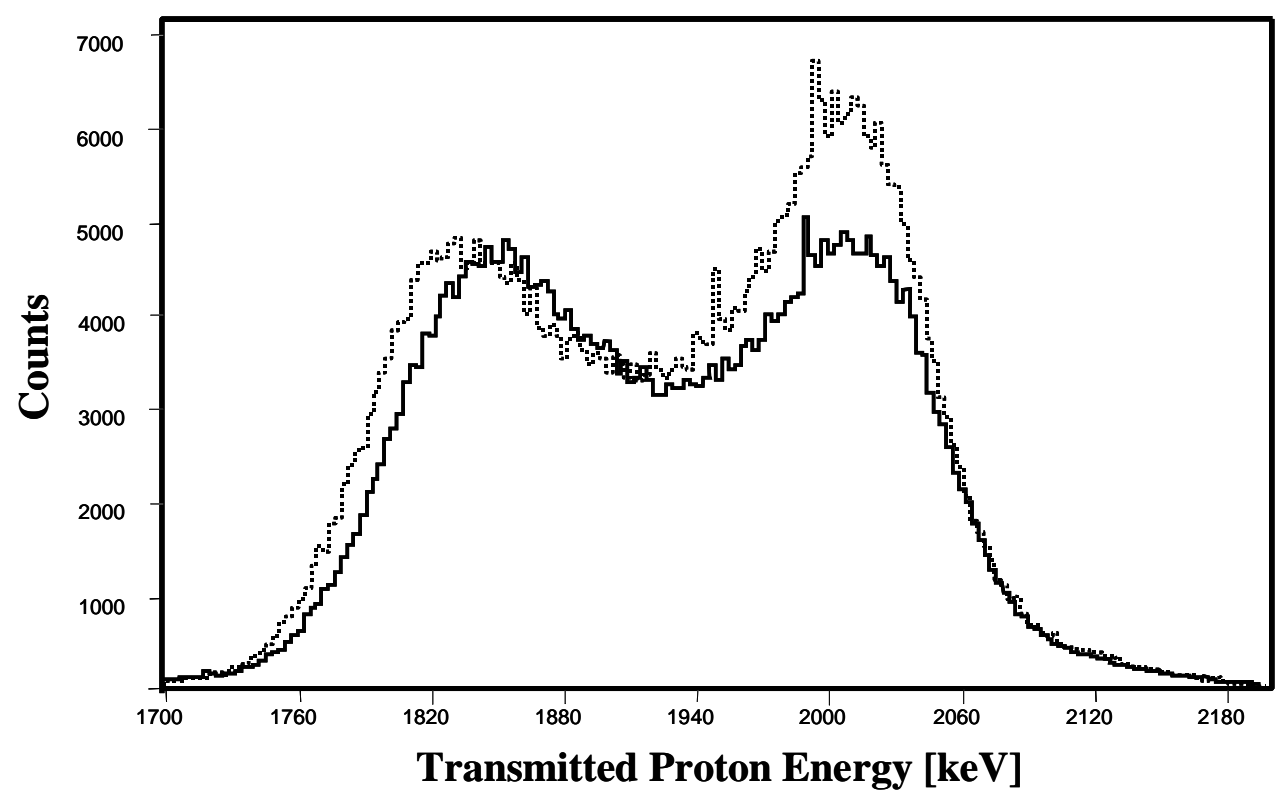

Figure 6-17: Transmission channeling spectra measured from the middle of the exfoliated region (dark area in figure 6-16 (a)) (solid line) and from the surrounding, non-blistered material (dotted line).

The spectra both exhibit two peaks. The lower energy peak is from those protons, which have a high rate of energy loss, corresponding to non-channeled trajectories. The higher energy peak corresponds to those protons, which have remained channeled through the $40 \mu \mathrm{m}$ thick sample. The spectrum from the exfoliated area has a non-channeled peak, which is $14 \mathrm{keV}$ higher in transmitted energy than that of the spectrum, obtained from a non-blistered sample area. This shift is due to the thinner sample thickness at the exfoliated region compared to the sample thickness at non-blistered sample areas.

The thickness difference equals the depth where surface blistering occurred. When non-channeled protons traverse the sample at non-blistered regions, they penetrate additional material compared to ions traversing blistered areas. They do so at an energy of approximately $1.84 \mathrm{MeV}$, the energy with which they leave the sample (see energy peak of non-channeled ions). Thus, the stopping power the non-channeled ions have during the pass through of the additional material is $5.67 \mathrm{eV} \mathrm{cm}^{2} / 10^{15}$ atoms (deduced from see figure 4-8, p. 38). This corresponds to a stopping power of $\mathrm{dE} / \mathrm{dx}=30.1 \mathrm{eV} / \mathrm{nm}$. Hence, the sample thickness difference, which causes the $14 \mathrm{keV}$ energy shift of the nonchanneled ion peak, is calculated to be $465 \mathrm{~nm}$. 
Assuming that the Ion-Cut takes place in the region of maximum damage and (100) $\mathrm{H}-$ platelet density, the thickness difference is expected to be about $420 \mathrm{~nm}$ (see figure 6-6 and 6-13), $45 \mathrm{~nm}$ shallower than predicted by the calculation based on the energy shift of the non-channeled ion peak. Hence, this comparison revealed that the shift in the positions of this left-hand peak in figure 6-17 does not exactly match the proton energy loss through the thickness of the exfoliated layer. This is owed to the contributing effect the larger channeled peak and the detector collimation have on the position of the nonchanneled peak.

The second important aspect is seen by comparing the relative heights of the two peaks. In the absence of any other mechanism occurring during exfoliation, the release of the highly compressed hydrogen gas, the absence of a secondary surface at the bubble base, and a slightly thinner sample would be expected to produce a more pronounced channeling peak in the transmission spectrum obtained from the blistered surface area compared to the spectrum taken from the surrounding material, since these dechanneling mechanisms have been removed. However, the channeling peak in the spectrum from the blistered region has a channeled peak, which is $26 \%$ smaller than that of the spectrum from the surrounding material. This implies that the crystalline quality at the base of the exfoliated bubble is considerably poorer than that of the surrounding, non-exfoliated silicon, although the material in blister regions does not contain the implantation damage, which is left in the exfoliated layer. Similar observations were made on the other exfoliated blisters shown in figure 6-15 (a).

An additional mechanism must therefore be occurring during exfoliation to produce the extra dechanneling. The data suggest, that the violent exfoliation process of the surface blister formation produces mechanically induced lattice damage on the lower surface, i. e. the subsurface region at the surface blister bottoms, and by extension, also on the upper separated surface. That means, when implanted to low H-doses, exfoliation increases the silicon crystal damage beyond the damage amount caused by the ionirradiation process and $\mathrm{H}$-complex formation.

Finally, it should be noted that since the blister density in the analyzed sample is very low, channeling measurements in backscattering geometry and with the use of an unfocused He-ion beam did not show a high dechanneling yield at higher channel numbers (see figure 6-5, p. 137). 


\section{Boron + Hydrogen Co-Implantation}

The study in chapter 6 showed, that the presence of acceptor or donor dopants in the silicon substrate have a considerable influence on the kinetics in the formation of $\mathrm{H}$-platelets and $\mathrm{H}_{2}$-gas bubbles, which induce the Ion-Cut. Recent research has shown that B-ion implantation into silicon prior to the H-ion implantation leads to a significant reduction in the annealing temperature (and/or annealing time) required for the exfoliation of the silicon layer in the Ion-Cut process. [156] Although a tremendous amount of research on the boron-hydrogen interaction in silicon has been conducted, to date the underlying mechanisms behind the enhancement of the Ion-Cut due to the presence of boron are still unknown.

Earlier studies have revealed that boron impurities in silicon have a large affinity for interstitial hydrogen. [48,50] It is unclear whether this affinity requires the boron atoms to reside at interstitial sites (as is the case in as-implanted boron) or at a substitutional lattice sites (as is the case after "activation" by rapid-thermal-annealing). It is known, however, that the boron atoms act as multiple traps for hydrogen. [49]

The aim of the present study is to explore the influence of boron in n-type $\langle 100\rangle$ silicon on the coalescence of hydrogen, formation of $\mathrm{H}_{2}$-gas bubbles, and finally surface blistering. Hence, silicon wafers were co-implanted with boron and hydrogen. In this study the boron and hydrogen implantation profiles overlap. To investigate the relevance of the boron lattice location (substitutional versus interstitial) on the H-bubble nucleation process, both as-implanted and activated samples are studied. To ensure the overlap between the implanted hydrogen and boron distributions, the boron was implanted deeper and over a broader distribution. The fact that the peaks in the implanted densities were separated made it also possible to investigate the range of the boron-hydrogen interaction.

The substrates used in this study were $<100\rangle$ oriented n-type silicon wafers with four different dopant levels, ranging from $10^{14} \mathrm{~cm}^{-3}$ to $10^{19} \mathrm{~cm}^{-3}$. The resistivities of the wafers and the corresponding donor dopant concentrations are listed in Table 7-1. 


\begin{tabular}{|ll|l|l|}
\hline \multicolumn{2}{|c|}{ Resistivity } & Dopant Concentration & Dopant Species \\
\hline \hline $0.050-0.020$ & $\Omega \mathrm{cm}$ & $1.3 \times 10^{18}-9.0 \times 10^{18} \mathrm{~cm}^{-3}$ & Phosphorous \\
\hline $0.03-1.0$ & $\Omega \mathrm{cm}$ & $4.5 \times 10^{15}-5.5 \times 10^{17} \mathrm{~cm}^{-3}$ & Phosphorous \\
\hline $1-10$ & $\Omega \mathrm{cm}$ & $4.5 \times 10^{14}-4.5 \times 10^{15} \mathrm{~cm}^{-3}$ & Phosphorous \\
\hline $30-50$ & $\Omega \mathrm{cm}$ & $8.0 \times 10^{13}-1.5 \times 10^{14} \mathrm{~cm}^{-3}$ & Phosphorous \\
\hline
\end{tabular}

Table 7-1: N-type <100> silicon substrates used in this study

Four implanted specimens were prepared for each of the four n-type dopant levels. The silicon substrates were first implanted with $240 \mathrm{keV} \mathrm{B}^{2+}$ ions to a dose of $1 \times 10^{15} \mathrm{~cm}^{-2}$. After the B-ion implantation the samples were either left in the as-implanted state or underwent a rapid thermal anneal (RTA) at $900^{\circ} \mathrm{C}$ for 30 and 60 seconds, respectively, to force the boron atoms into substitutional lattice positions, thereby electrically activating the boron atoms. The samples were then implanted with $40 \mathrm{keV} \mathrm{H} \mathrm{H}^{+}$ions to a dose of $5.0 \times 10^{16} \mathrm{~cm}^{-2}$. To evaluate the role of the $\mathrm{B}^{+}$implantation, control samples were prepared by implanting with $\mathrm{H}^{+}$ions only. Following the $\mathrm{H}^{+}$implantation, all the samples underwent a vacuum anneal at $390{ }^{\circ} \mathrm{C}$ for 10 minutes. The base vacuum in both annealing treatments was $5 \times 10^{-8}$ Torr. All ion implants were performed at the liquid nitrogen temperature of $77 \mathrm{~K}$. To minimize channeling effects during implantation, the incident ion beam was oriented at 7 degree from the surface normal. The sample preparations by the described sequence of ion implant and anneals are summarized in Table 7-2.

\begin{tabular}{|c|c|c|c|c|}
\hline $\begin{array}{c}\text { Sample } \\
\text { Identifier }\end{array}$ & $\begin{array}{c}\text { Boron Implantation } \\
\text { Dose and Energy }\end{array}$ & $\begin{array}{c}\text { First Anneal: Rapid } \\
\text { Thermal Anneal } \\
\text { (in Vacuum) }\end{array}$ & $\begin{array}{c}\text { Hydrogen } \\
\text { Implantation Dose } \\
\text { and Energy }\end{array}$ & $\begin{array}{c}\text { Second Anneal } \\
\text { (in Vacuum) }\end{array}$ \\
\hline \hline A & $\begin{array}{c}1 \times 10^{15} \mathrm{~cm}^{-2} \\
240 \mathrm{keV}\end{array}$ & --- & $\begin{array}{r}5 \times 10^{16} \mathrm{~cm}^{-2} \\
40 \mathrm{keV}\end{array}$ & $10 \min @ 390^{\circ} \mathrm{C}$ \\
\hline B & $\begin{array}{c}1 \times 10^{15} \mathrm{~cm}^{-2} \\
240 \mathrm{keV}\end{array}$ & $30 \mathrm{sec} @ 900^{\circ} \mathrm{C}$ & $\begin{array}{r}5 \times 10^{16} \mathrm{~cm}^{-2} \\
40 \mathrm{keV}^{-2}\end{array}$ & $10 \min @ 390^{\circ} \mathrm{C}$ \\
\hline $\mathrm{C}$ & $\begin{array}{c}1 \times 10^{15} \mathrm{~cm}^{-2} \\
240 \mathrm{keV}\end{array}$ & $60 \mathrm{sec} @ 900^{\circ} \mathrm{C}$ & $\begin{array}{c}5 \times 10^{16} \mathrm{~cm}^{-2} \\
40 \mathrm{keV}^{-2}\end{array}$ & $10 \min @ 390^{\circ} \mathrm{C}$ \\
\hline $\mathrm{D}$ & --- & -- & $\begin{array}{c}5 \times 10^{16} \mathrm{~cm}^{-2} \\
40 \mathrm{keV}^{--}\end{array}$ & $10 \min @ 390^{\circ} \mathrm{C}$ \\
\hline
\end{tabular}

Table 7-2: Processing used to prepare four samples from each of the n-type dopant level silicon wafers. The process for each sample is read from left to right. 
The B-ion implantation dose was chosen so that, for low n-type doped silicon substrates, the boron concentration induces a transition of the conductivity type from n- to p-type in the depth region where the hydrogen ions come to rest, but leaves the conductivity type unchanged in the highest n-type doped silicon substrate. This made it possible to investigate the influence of the conductivity type on surface blister formation.

The choice of the B-ion-implantation dose was based on TRIM simulations [75]. Figure 7-1 shows the predicted depth profiles of the $\mathrm{H}$ and $\mathrm{B}$-concentrations in the material after the ion-implantations. Overlaid are also the calculated damage profiles caused by the two implantation processes.

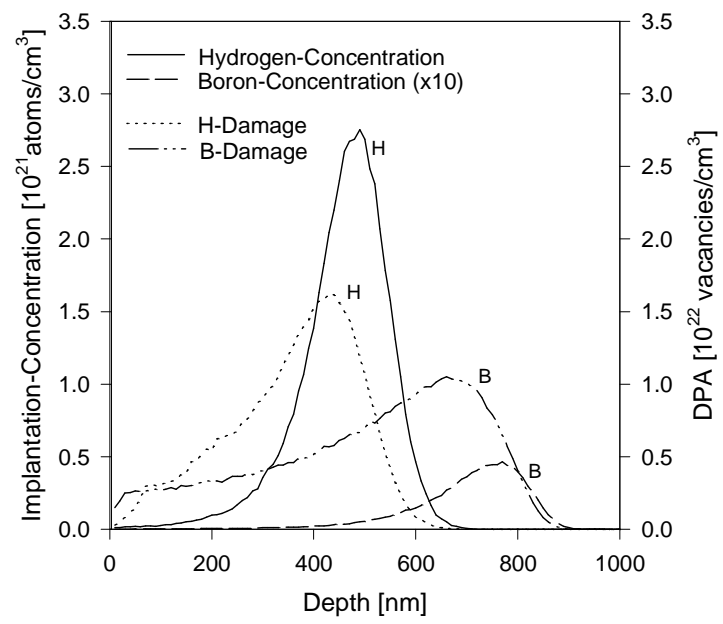

Figure 7-1: TRIM calculation [75] of the ion-distribution and the implantation induced damage distribution in silicon for $40 \mathrm{keV} \mathrm{H}$-ions at a dose of $5 \times 10^{16} \mathrm{~cm}^{-2}$, and $240 \mathrm{keV} \mathrm{B}$ ions at a dose of $1 \times 10^{15} \mathrm{~cm}^{-2}$, respectively.

The simulations show that, in the depth region of maximum hydrogen concentration, the boron concentration has a value of about $2 \times 10^{18} \mathrm{~cm}^{-3}$. A comparison of this value with the n-type background dopant level of the sample with the lowest resistivity (Table 7-1) shows, that the conductivity type in the depth region of the H-distribution is expected to remain unchanged for this silicon substrate.

The temperature and duration for the second anneal were selected such that the various samples show large differences in the surface blister densities. This makes it possible to elucidate the enhancing or suppressing effects of the various sample preparation parameters like boron pre-implantation or electrical boron activation. Would the anneal temperature and/or duration be significant lower, no sample would form surface blisters, whereas a significant increase in annealing temperature and/or duration would result in intense surface blistering for all samples. 


\subsection{Implantation Damage Depth Profiles}

To examine the influence the B-ion pre-implantation has on the formation of surface blisters, it is essential to gain knowledge about the damage accumulation, caused by the ion implantation processes. Rutherford Backscattering Spectroscopy (RBS) in the channeling mode was used to analyze the radiation damage caused by both the boron and hydrogen doping. All RBS channeling measurements were carried out with $2.0 \mathrm{MeV}$ helium $(\alpha)$ analyzing beam.

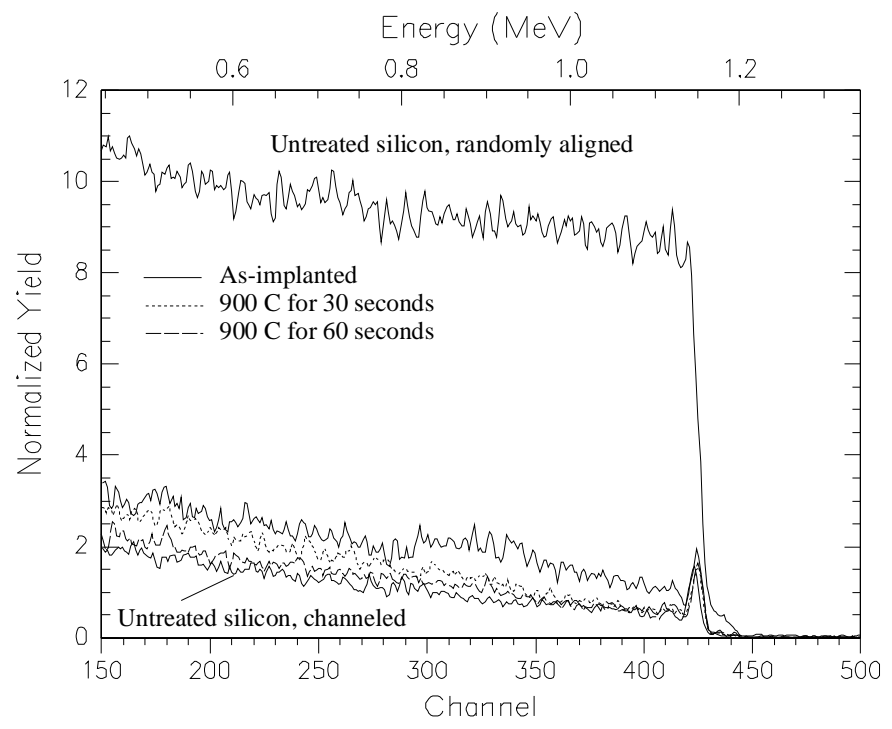

Figure 7-2: $2.0 \mathrm{MeV}$ He RBSchanneling spectra of the <100> n-type $\mathrm{Si}$ sample with a resistivity of $0.005-0.020 \Omega \mathrm{cm}$ after the B-ion implantation, but before the subsequent $\mathrm{H}$-ion implantation. The graph shows the spectra from the asimplanted sample (solid line), after activation at $900{ }^{\circ} \mathrm{C}$ for 30 seconds (dotted line) and 60 seconds (dashed line). For comparison a random spectrum and the channeling spectrum of the untreated crystal are shown, too.

Figure 7-2 presents channeling RBS spectra obtained from the samples with a resistivity of $0.005-0.20 \Omega \mathrm{cm}$ after the B-ion implantation either in the as-implanted state of after electrical activation by the two different thermal treatments. The data show, that the Bion implantation causes only little damage accumulation in the sample: The direct backscattering yield in the as-implanted sample, extending from channel 280 to 420 , shows a maximum backscattering yield $\chi_{\max }$ of $13 \%$. After annealing at $900{ }^{\circ} \mathrm{C}$ for 30 seconds the direct backscattering peak is not visible any more, indicating that a high percentage of the implanted B-atoms occupied a substitutional lattice site and thus is electrical active. After annealing at $900{ }^{\circ} \mathrm{C}$ for 60 seconds the dechanneling yield also decreases and the RBS channeling yield equals almost the RBS channeling yield from the untreated silicon substrate.

The RBS channeling analysis shows a $40 \%$ lower damage accumulation in the silicon sample by the B-ion implantation than predicted by the simulation program TRIM 
(see figure 7-1). The B-ion implantation was performed at room temperature, allowing for damage recovery during the ion-implantation process. This effect is not accounted for in the TRIM simulation. The difference between the simulated and the measured implantation damage reveals that about $40 \%$ of the ion-irradiation induced damage recovers during the ion-implantation process.

RBS channeling measurements were also carried out on all samples after the $\mathrm{H}$ ion implantation. The channeling data were obtained prior to the second thermal annealing step. Figure 7-3 shows, as an example, channeling RBS spectra obtained on all samples with a resistivity of $1-10 \Omega \mathrm{cm}$ and $30-50 \Omega \mathrm{cm}$. The peaks located between channel 340 and channel 390 indicates lattice damage caused by the H-ion implantation. This damage is quantified by backscattering factors. The direct backscattering yield $\mathrm{D}$ is obtained by subtracting the baseline from the channeling yield caused by the $\mathrm{H}$ implantation damage. The baseline within the $\mathrm{H}$-implantation damage region is simply chosen as a straight line joining the yield immediately outside this region (linear approximation) as shown in the right part of figure 7-3 for the sample labeled [B (60sec RTA) + H]. (see also chapter 4.2.1, figure 4-16, p. 48).
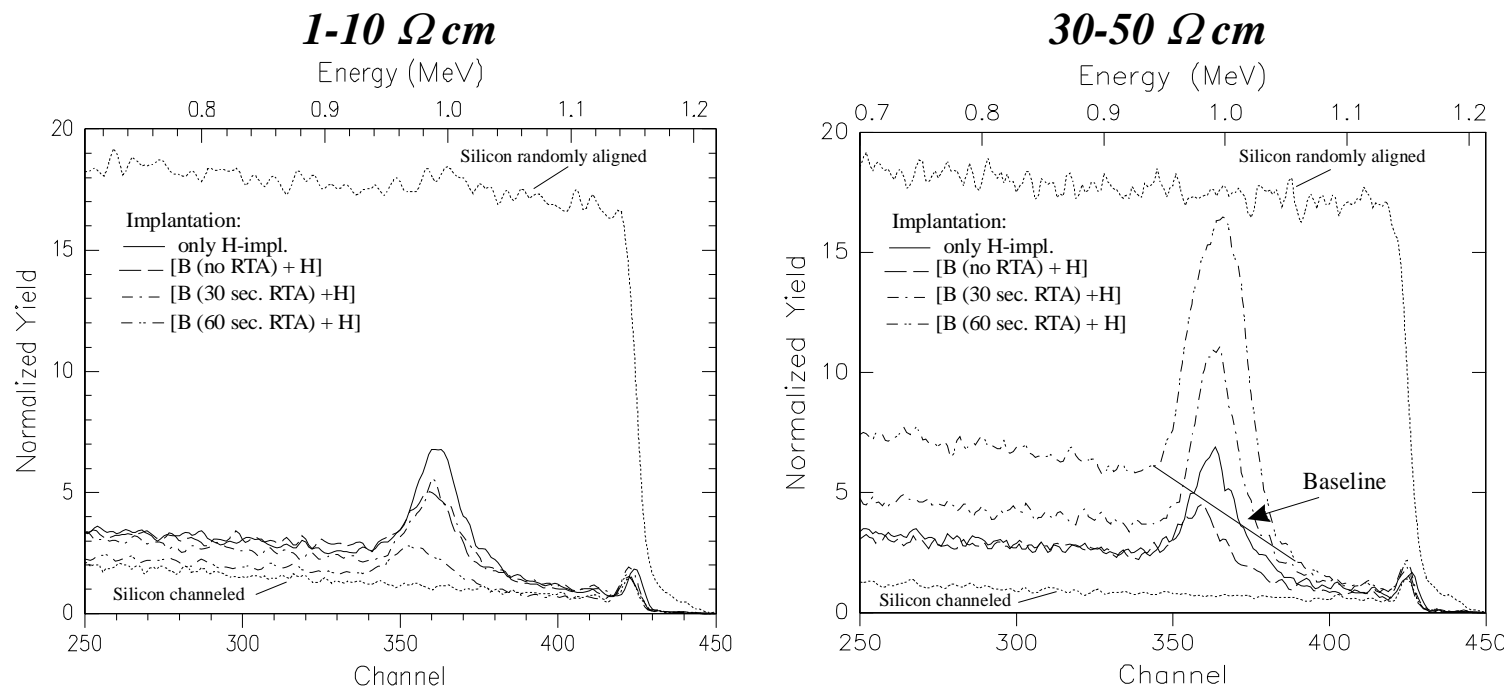

Figure 7-3: $2.0 \mathrm{MeV}$ He RBS-channeling spectra of the <100> n-type silicon samples with a resistivity of 1-10 $\Omega \mathrm{cm}$ and 30-50 $\Omega \mathrm{cm}$, respectively, and different treatments. For comparison a random spectrum and the channeling spectrum of the untreated crystal are shown, too. As an example, the linear approximation of the baseline for the [B $(60 \mathrm{sec}$. RTA $)+\mathrm{H}]$ - implanted sample is also shown.

The RBS channeling spectra show clearly, that the damage accumulation in the material is significantly dependent on the n-type background dopant level and the degree of electrical activation of the boron implant. 
I further discuss the normalized direct backscattering yield $D_{n}=D /\left(Y_{\text {random}}-Y_{\text {channeled }}\right)$, integrated over the depth region of the $\mathrm{H}$-implantation damage (channel region 340 - 390). Notice that samples with a low concentration of defects in this depth region result in samples with low $D_{n}$ values. Figure 7-4 shows $D_{n}$ values for all the samples used in this study as a function of the n-type dopant level. Each datum in this figure is the average of at least two independent RBS measurements carried out on different surface areas of each sample. Error bars are not shown because they are smaller than the symbol size in this figure.

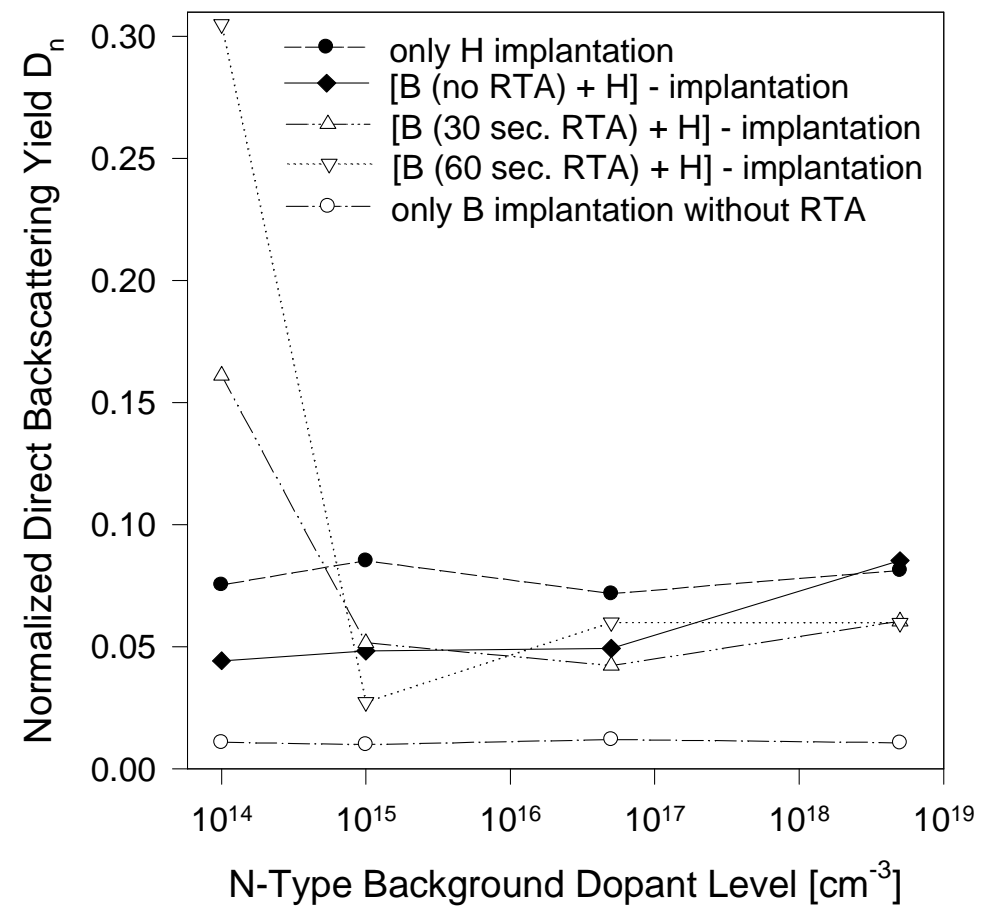

Figure 7-4: Normalized direct backscattering yield $D_{n}$ versus background dopant level for all four sample treatments. The data were acquired prior to second annealing. The lines serve only as a guide to the eye.

Samples implanted with hydrogen only show no dependence of $D_{n}$ on the n-type dopant level. Samples that were implanted with both boron and hydrogen, but for which boron was not activated prior to the hydrogen-implantation ( $\$$-symbol data), have $D_{n}$ values that are lower than the sum of the $D_{n}$ values for the boron-implanted and the hydrogenimplanted samples (sum of the $\mathbf{O}+\mathrm{O}$ data). Furthermore, the data indicate a decrease in $\mathrm{D}_{\mathrm{n}}$ of up to $50 \%$ at low background dopant level compared to $\mathrm{D}_{\mathrm{n}}$ of the samples implanted with $\mathrm{H}$ only; $\mathrm{D}_{\mathrm{n}}$ increases with increasing n-type background dopant level, until reaching the $D_{n}$ value of the corresponding sample implanted with hydrogen only. The rapid thermal annealing following the boron-implantation seems to have a drastic effect on $D_{n}$, but only for the samples with the lowest n-type dopant level of about 
$10^{14} \mathrm{~cm}^{-3}$. For higher n-type dopant levels, the damage of the boron-implanted and activated samples is lower than in similar samples implanted with hydrogen only.

Previous experiments have shown that the presence of boron reduces the damage accumulation caused by ion implantation in silicon. [153] This explains why $D_{n}$ for the $[B($ no RTA $)+H]$ - implanted samples is lower than $D_{n}$ in the H-only implanted samples (figure 7-4). For high implantation doses, radiation damage leads to the formation of amorphous domains or islands. Boron has been shown to significantly reduce the growth rate of the amorphous islands but not to have an influence on the nucleation rate of these islands. [153] N-type dopants in $\mathrm{Si}$ on the other hand have been shown to enhance the growth of small pre-existing amorphous clusters. [157] The competition between these effects explains the trends observed in figure 7-4. Indeed, for all samples, the boron concentration in the region of the hydrogen distribution (400-500 $\mathrm{nm}$ depth) has the same value of about $2 \times 10^{18} \mathrm{~cm}^{-3}$ (see figure 7-1). Accordingly, the reduction in the growth rate of the amorphous phase is the same for all samples. However, for a constant boron radiation dose, the growth rate of the amorphous phase increases with increasing n-type dopant level. [157] At the n-type background dopant level of about $5 \times 10^{18} \mathrm{~cm}^{-3}(0.005$ $0.020 \Omega \mathrm{cm}$ ) the n-type doping exceeds the p-type doping induced by the boron implantation, and the retained implantation damage (mainly induced by the hydrogenimplantation) reaches the same value as in the sample which was implanted with hydrogen only. At high n-dopant levels the implanted boron has little or no effect on reducing the damage accumulation and amorphous phase development.

RBS channeling calculations revealed furthermore, that, for all sample treatments, the region of maximum damage is located at the depth of $405 \mathrm{~nm}+/-5 \mathrm{~nm}$. Thus, the n-type background dopant level or the pre-implanted boron and its electrical state do not influence the depth of the implantation damage peak.

Upon the second thermal anneal surface blister form in most of the sample, making an accurate RBS channeling analysis of the remaining implantation damage impossible: Unlike in non-blistered surface regions, in areas, where the wafer surface blisters, the blister bottom is exposed and consequently becomes the depth, where the analyzing beam starts to penetrate the material. Thus, the implantation damage in blistered areas produces a large backscattering and dechanneling yield over the whole energy range of the backscattered ions. As an example, figure 7-5 shows such a RBS channeling spectrum. It is not possible to determine the baseline and consequently the direct backscattering yield, which corresponds to the buried damage layer. 


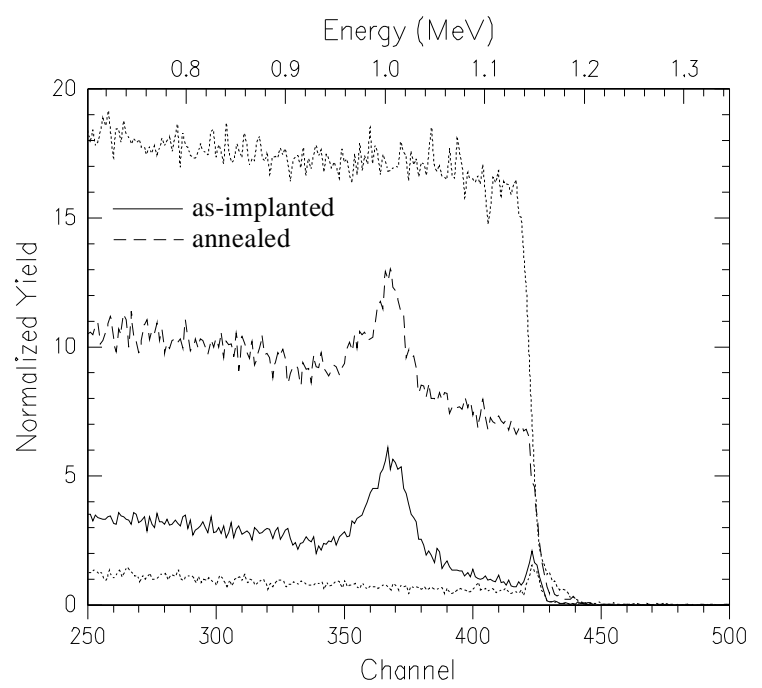

Figure 7-5: $2.0 \mathrm{MeV}$ He RBSchanneling spectra of the $\langle 100\rangle \mathrm{Si}$ sample with a resistivity of $0.03-1 \Omega \mathrm{cm}$. The sample was [B (60sec. RTA) + H] - implanted. The spectrum was obtained before and after the second annealing at $390{ }^{\circ} \mathrm{C}$ for 10 minutes. For comparison a random spectrum and the channeling spectrum of the virgin crystal are shown, too (dotted lines).

\subsection{Hydrogen and Boron Implantation Depth Profiles}

To investigate the influence the B-ion pre-implantation on the formation of surface blisters in the $\mathrm{H}$-ion implanted samples, it is crucial to obtain accurate information about the depth profiles of the implanted boron and hydrogen.

To obtain high accuracy in the assessment of the hydrogen and boron depth distribution, a combination of ERD analysis and SIMS was employed. ERD analysis allows a precise measurement of the amount of implanted hydrogen, remaining in the silicon samples, and the depth of the H-concentration peak. SIMS on the other hand enables an evaluation of the shape of the hydrogen depth profiles to a precision not achievable by ERD analysis due to the energy straggling of the ERD analyzing beam. Furthermore, SIMS allows for the determination of the B-concentration depth profiles.

The ERD measurements were performed using a $2.2 \mathrm{MeV}$ He beam. A $10 \mu \mathrm{m}$ thick Mylar foil was positioned between the sample and the hydrogen detector to avoid the detection of backscattered He-ions. ERD measurements of the H-depth distribution were carried out on all samples. Figure 7-6 shows, as an example, the ERD spectra 
obtained for the sample with a n-type dopant level of about $10^{14} \mathrm{~cm}^{-3}$ and sample treatment B (see Table 7-2) before and after the second anneal treatment.

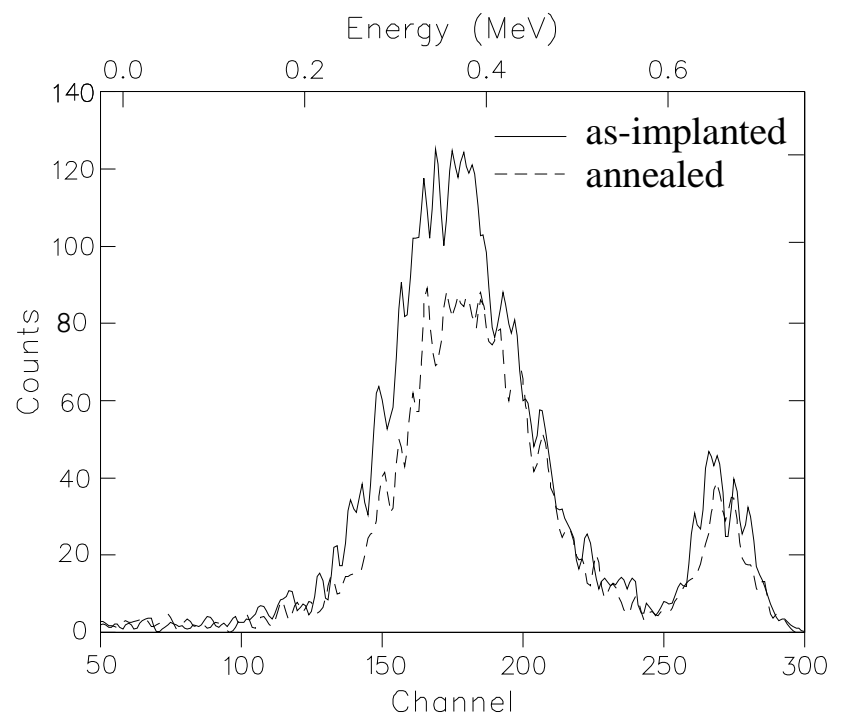

Figure 7-6: ERD-spectra of a $<100>\mathrm{n}$ type Si sample with a resistivity of $30-50$ $\Omega \mathrm{cm}$ implanted with $40 \mathrm{keV} \mathrm{H}$ ions to a dose of $5 \times 10^{16} \mathrm{~cm}^{-2}$. Prior the hydrogenimplantation $240 \mathrm{keV}$ B ions were implanted at a dose of $1 \times 10^{15} \mathrm{~cm}^{-2}$ and activated by a rapid thermal anneal at $900^{\circ} \mathrm{C}$ for 30 seconds. The spectrum was obtained from the as-implanted sample (solid line) and after the sample underwent the final anneal at $390{ }^{\circ} \mathrm{C}$ for ten minutes (dashed line).

The small peak near channel 270 originates from hydrogen absorbed on the sample surface while the peak near channel 170 originates from the implanted hydrogen. The depth of the H-concentration peak in the as-implanted sample was calculated to be $435 \mathrm{~nm}$. Figure 7-6 clearly shows that, within the resolution of the method, there is no detectable movement of hydrogen towards the deeper boron peak (towards lower channel number) during the second anneal. However, the ERD signal between channels 140 and 180 after the second anneal shows a reduction in total integrated counts of the forward scattered signal at lower energies, reflecting a decrease of the hydrogen content in the surface far side of the H-depth distribution. This is in part a result of the loss of hydrogen due to the surface blistering that takes place during the second anneal. A small amount of hydrogen remains in the "popped-off" blister covers and consequently the hydrogen content remaining in the sample is slightly reduced. In addition, hydrogen in form of $\mathrm{H}_{2}$ gas in the sub-surface bubbles, which lead to ion-cutting during annealing, are liberated in the moment surface blistering occurs.

It is unclear however, if diffusion of hydrogen either into the bulk material or towards the surface contributes to the hydrogen loss. Eliminating the effect of surface blistering on the ERD spectra would enable the examination of the hydrogen diffusion behavior. Therefore, additional samples with a lower $\mathrm{H}$-ion implantation dose were prepared such that no surface blistering occurs upon annealing at the present anneal temperature and duration. These samples were prepared for all four n-type silicon 
substrates in the same manner as sample treatment A and D ([B (no RTA) $+\mathrm{H}]$ and $\mathrm{H}-$ only implantation, see Table 7-2), but with a lower $\mathrm{H}$-implantation dose of $2.5 \times 10^{16} \mathrm{~cm}^{-2}$.

Figure 7-7 shows the ERD spectra of the sample implanted with $\mathrm{H}$-ions only to a dose of $2.5 \times 10^{16} \mathrm{H} / \mathrm{cm}^{2}$, either in the as-implanted state or after annealing. The n-type silicon substrate has a resistivity of $0.05-0.20 \Omega \mathrm{cm}$.

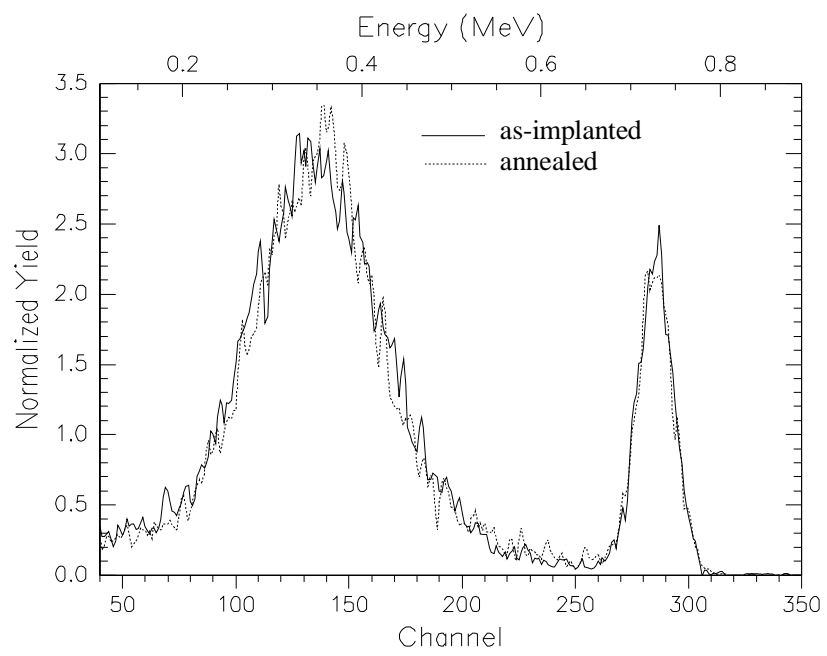

Figure 7-7: ERD-spectra of a <100> ntype $\mathrm{Si}$ sample with a resistivity of 0.05-0.20 $\Omega \mathrm{cm}$ implanted with $40 \mathrm{keV} \mathrm{H}$ ions to a dose of only $2.5 \times 10^{16} \mathrm{~cm}^{-2}$. The spectrum was obtained from the asimplanted sample (solid line) and after the sample underwent the final anneal at $390{ }^{\circ} \mathrm{C}$ for ten minutes (dotted line).

Annealing appears to have no ERD detectable influence on the H-depth distribution. This is in accordance with the detailed study of the H-diffusion behavior in chapter 6-1, where the $\mathrm{H}$-implantation dose was comparable to that one used here.

Figure 7-8 presents the ERD spectra of the as-implanted and annealed sample, which underwent pre-implantation of B-ions. A comparison of figure 7-8 with figure 7-7 reveals, that the B-ion pre-implantation has a significant effect on the diffusion behavior of the H-implant upon annealing. Since no surface blisters formed, the decrease of the hydrogen signal is due to diffusion of the H-implant. Examining figure 7-8 in more detail, it becomes apparent that the implanted hydrogen diffuses towards the bulk material: At low energies (channel 50 -100) the ERD signal of the annealed sample is higher than that from the as-implanted sample. 


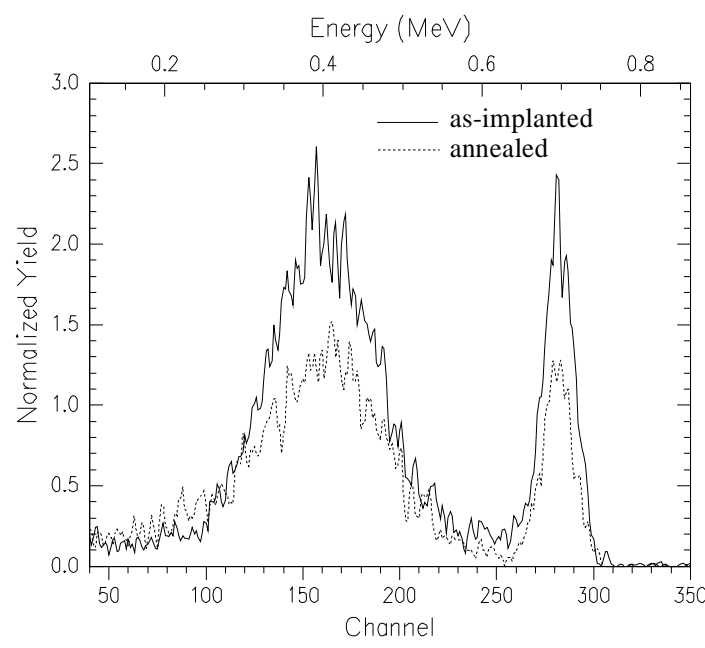

Figure 7-8: ERD-spectra of a $<100>$ n-type $\mathrm{Si}$ sample with a resistivity of $0.005-0.020 \Omega \mathrm{cm}$ implanted with $240 \mathrm{keV} \mathrm{B}$ ions to a dose of $1 \times 10^{15} \mathrm{~cm}^{-2}$ and subsequently with $40 \mathrm{keV} \mathrm{H}$ ions to a dose of $2.5 \times 10^{16} \mathrm{~cm}^{-2}$. The spectrum was obtained from the as-implanted sample (solid line) and after the sample underwent the final anneal at $390^{\circ} \mathrm{C}$ for ten minutes (dotted line).

The ERD spectra of the $\mathrm{H}$-only implanted or the $\mathrm{B}+\mathrm{H}$ implanted samples looked identical for all four different n-type dopant levels. This indicates that the n-type dopant level does not have a significant influence on the long-range diffusion of the H-implant.

To acquire more detailed information about the shape of the H-depth distribution, SIMS measurements were carried out on all samples implanted with low dose hydrogen. The SIMS spectra were calibrated using the ERD analysis results: The time axes in the SIMS spectra were transformed into depth scales, such that the depths of maximum $\mathrm{H}$ concentration equal the H-concentration peaks depths, determined by ERD. The magnet, diverting the $\mathrm{H}$-ions into the mass spectrometer during the SIMS measurements, had to be readjusted after each measurement. Consequently, the amount of detected H-counts did not represent the absolute value of the actual $\mathrm{H}$-concentration. Therefore, the $\mathrm{y}$-axes in the SIMS spectra, i. e. the detected H-counts, were adjusted so that the amount of the $\mathrm{H}$-counts, integrated over the whole depth distribution is normalized to the H-amount in the samples, determined by ERD analysis. The implanted boron on the other hand showed no long range diffusion and therefore, the height of the boron signal was adjusted to fit the known implantation dose of $1 \times 10^{15} \mathrm{~cm}^{-2}$. The samples were characterized in detail before and after the final annealing.

Figure 7-9 shows the adjusted SIMS spectra of the as-implanted and annealed sample, implanted with hydrogen only to the low dose of $2.5 \times 10^{16} \mathrm{~cm}^{-2}$. The n-type silicon substrate resistivity was $0.005-0.020 \Omega \mathrm{cm}$. 


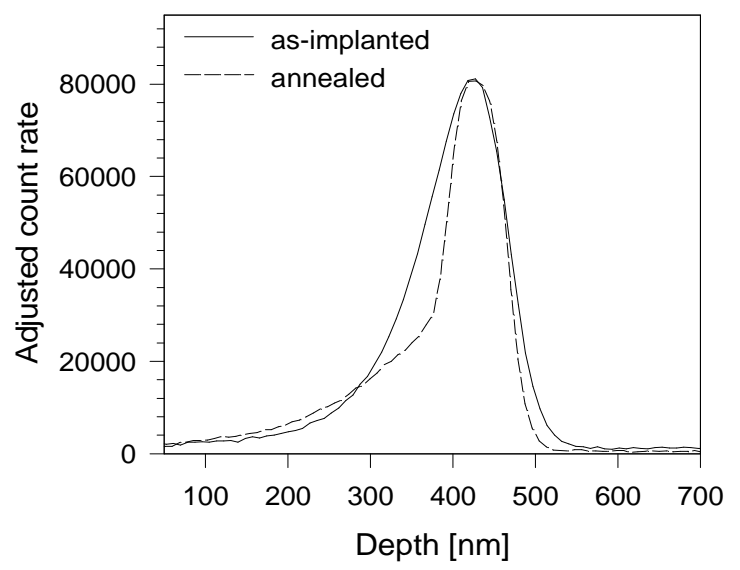

Figure 7-9: Adjusted SIMS spectra of the hydrogen depth distributions after $\mathrm{H}$-ion implantation at $40 \mathrm{keV}$ to a dose of $2.5 \times 10^{16} \mathrm{~cm}^{-2}$. The n-type silicon substrate had a resistivity of $0.005-0.020 \Omega \mathrm{cm}$. The $\mathrm{H}-$ depth distribution was monitored in the asimplanted state and also after annealing at $390{ }^{\circ} \mathrm{C}$ for 10 minutes.

As in chapter 6-1, the SIMS data show self-trapping of hydrogen in the region of maximum H-concentration and some out-diffusion of the hydrogen located at shallower depths. This phenomenon is due to the formation of immobile $\mathrm{H}_{2}$ molecules formation in regions of high $\mathrm{H}$-concentration and is discussed in detail in chapter 6-1.

The adjusted SIMS spectra from samples, undergoing sample treatment A (B (no RTA) + H implantation), but with a lowered $\mathrm{H}$-ion implantation dose of $2.5 \times 10^{16} \mathrm{~cm}^{-2}$ are presented in figure 7-10. As in the figure 7-9, the n-type silicon substrate resistivity was $0.005-0.020 \Omega \mathrm{cm}$.
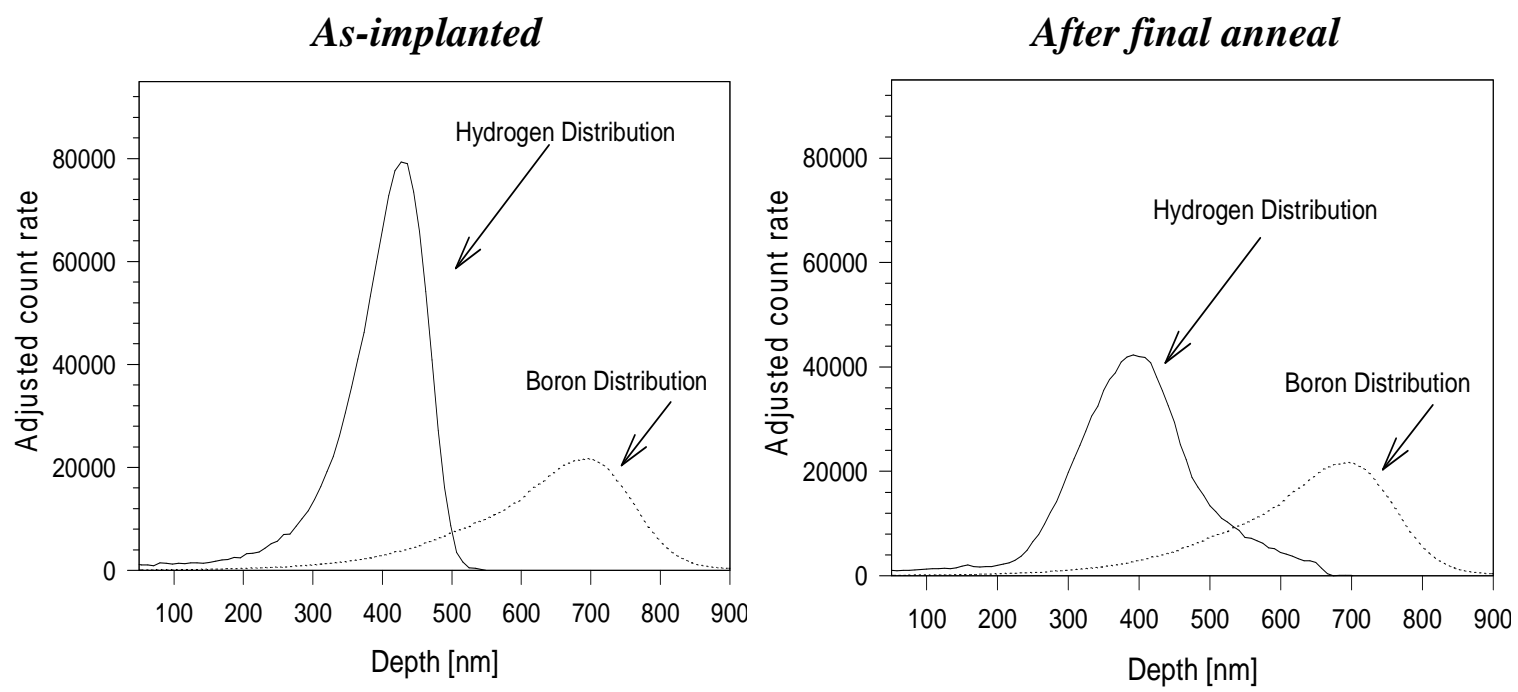

Figure 7-10: Adjusted SIMS spectra of the boron and hydrogen depth distributions in the [B (no RTA) $+\mathrm{H}$ ] implanted sample. The n-type silicon substrate had a resistivity of $0.005-0.020 \Omega \mathrm{cm}$. The implanted $\mathrm{H}$-dose was reduced to $2.5 \times 10^{16} \mathrm{~cm}^{-2}$ to avoid surface blistering upon annealing at $390{ }^{\circ} \mathrm{C}$ for 10 minutes. To make the boron depth distribution more visible, the adjusted heights of the boron signals were multiplied by a factor of 10 .

Comparing the SIMS spectrum from the as-implanted sample with the one in figure 7-9, the plot reveals that the boron pre-implant does not have an effect on the H-concentration 
depth distribution. The shape and depth of the H-concentration profile is equal to that shown in figure 7-9, where no boron is present in the sample. This observation is in accordance with above ERD analysis on as-implanted samples. However, after the second anneal, the H-depth distribution differs significantly from that in the H-only implanted sample. Whereas $\mathrm{H}$-self trapping in the region of maximum $\mathrm{H}$-concentration was observed, when no boron is present, the SIMS measurements on the $\mathrm{B}+\mathrm{H}$ coimplanted and annealed sample (right part of figure 7-10) reveals that considerable diffusion of the H-implant towards the bulk, i.e. towards higher boron concentrations, occurs.

The reduction of the $\mathrm{H}$ self-trapping in the $\mathrm{H}$-concentration peak can be caused by a long range interaction between the $\mathrm{H}$-implant and the deeper $\mathrm{B}$-concentration peak, a short range interaction of the boron located in the depth region of highest hydrogen concentration, or both. To investigate the observed phenomenon more, $[$ B (no RTA $)+$ H] - implanted silicon samples were prepared similar to the samples studied above with a lower H-dose of $2.5 \times 10^{16} \mathrm{~cm}^{-2}$, but with a B implantation energy, such that the B-concentration peak is located in the depth region of maximum $\mathrm{H}$-concentration. The B-ion implantation energy was chosen based on TRIM [75] calculations, which predicted overlap of the $\mathrm{H}$ and $\mathrm{B}$ concentration peaks at a $\mathrm{B}$-ion energy of $147 \mathrm{keV}$. The samples were analyzed in the same manner as the samples studied above. Figure 7-11 presents the adjusted SIMS spectra from the sample in the asimplanted state and after annealing at $390^{\circ} \mathrm{C}$ for 10 minutes, respectively.
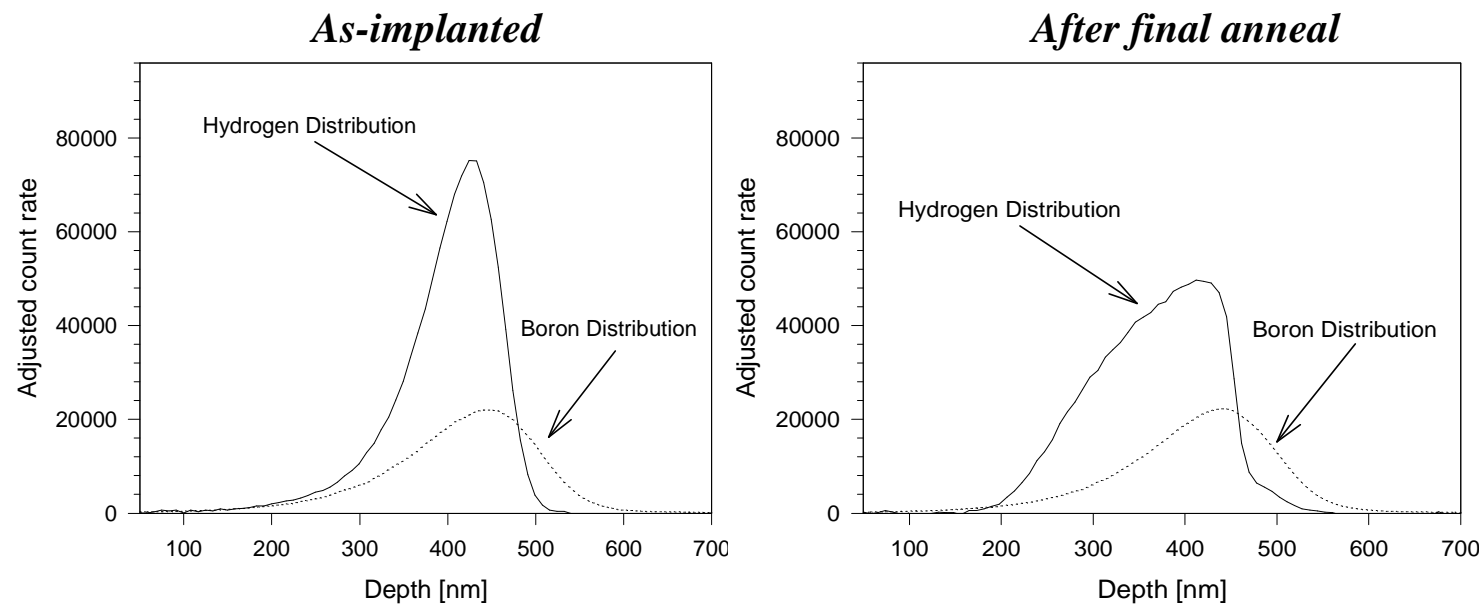

Figure 7-11: Adjusted SIMS spectra of the boron and hydrogen depth distributions in a silicon sample implanted with $147 \mathrm{keV}$ B-ions to a dose of $1 \times 10^{15} \mathrm{~cm}^{-2}$ followed by a $40 \mathrm{keV} \mathrm{H}$-ion implantation to a dose of $2.5 \times 10^{16} \mathrm{~cm}^{-2}$. The n-type silicon substrate had a resistivity of $0.005-0.020 \Omega \mathrm{cm}$. The SIMS spectra were obtained from the as-implanted sample and also after sample annealing at $390{ }^{\circ} \mathrm{C}$ for 10 minutes. To make the boron depth distribution more visible, the adjusted heights of the boron signals were multiplied by a factor of 10 . 
As in the high energy B-ion implanted sample, the boron implant does not have an influence on the H-depth distribution in the as-implanted state. However, when annealed the H-depth profile changed significantly. The data reveal, that the high boron concentration in the region of maximum $\mathrm{H}$-concentration leads to the annihilation of the self trapping of hydrogen at the region of maximum H-concentration. Nonetheless, the data also show, that some other trap mechanism suppresses hydrogen out-diffusion and causes the hydrogen to remain in the depth region of the B-implant. Thus, the SIMS measurements suggest, that the effect of boron in high energy B-ion pre-implantation sample (see figure 7-10) is caused by both, long-range interaction of the deep boron concentration peak with the $\mathrm{H}$-implant and short range interaction between the H-implant and the $\mathrm{B}$-atoms residing in the region of high $\mathrm{H}$-concentration. As mentioned earlier, the nature of these boron-hydrogen interactions are still unknown.

\subsection{SEM Analysis of the Surface Blistering}

To correlate the damage profiles of the as-implanted samples and the boron and hydrogen depth distributions in the as-implanted and annealed samples with the surface blister formation during the second anneal (see Table 7-2) SEM imaging was performed on all samples. The SEM study was carried out, looking both at the sample surface and in cross section. From these observations the average blister density and the average blister depth was deduced for each sample.

Figure 7-12 shows, as an example, a SEM image of the sample with a n-type dopant level of about $10^{14} \mathrm{~cm}^{-3}$ and sample treatment B (see Table 7-2) after the second annealing. Clearly evident are "popped-off" surface blisters (regions with white boundaries) as well as hydrogen-filled bubbles beneath the surface (darker spots). These bubbles have not yet evolved into the "popped-off" state. The average diameter of the surface blisters is about $5 \mu \mathrm{m}$. As mentioned in chapter 5.2 , the presence of surface blisters indicates the tendency of the implanted hydrogen to form microcracks parallel to the surface. Had the implanted wafer been bonded to another substrate prior to final annealing, the formation of individual popped off blisters would have been suppressed and the hydrogen induced microcracks would lead to the complete exfoliation of the 
surface layer instead (see also figure 5-21, p. 93). Although researchers have found that the annealing time required for such a delamination is much longer than that needed to form surface blisters, the activation energy is the same. [1]

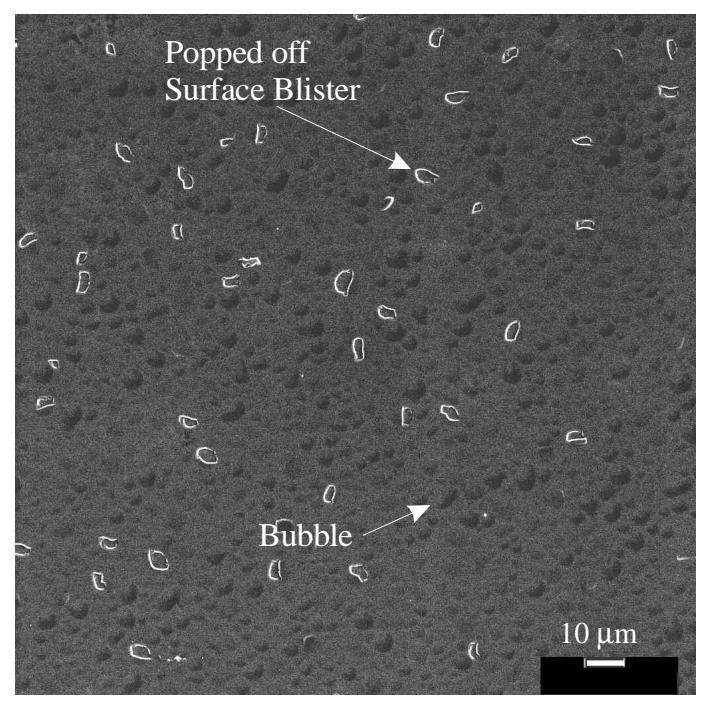

Figure 7-12: SEM image of the wafer surface of the $\langle 100\rangle$ n-type silicon sample with a resistivity of $30-50 \Omega \mathrm{cm}$ after the $[\mathrm{B}$ (RTA for 30 seconds at $\left.\left.900^{\circ} \mathrm{C}\right)+\mathrm{H}\right]$ implantation treatment and second annealing.

Figure 7-13 shows the result of a blister density analysis performed after the second anneal as a function of the substrate n-type background doping. There was no blister formation in the samples implanted with hydrogen only. The blister density for the $[\mathrm{B}+\mathrm{H}]$ co-implanted samples, where $\mathrm{B}$ was not activated prior to the hydrogenimplantation ( data), increases rapidly with increasing n-type background dopant level. However, samples where the boron was activated prior to the hydrogen-implantation $(\nabla$ and $\Delta$ data) show the opposite trend.

Figure 7-13: $\quad$ Surface blister density as a function of the background dopant level after second annealing at $390{ }^{\circ} \mathrm{C}$ for 10 minutes. The data were obtained by SEM. The lines are intended to highlight the trends.

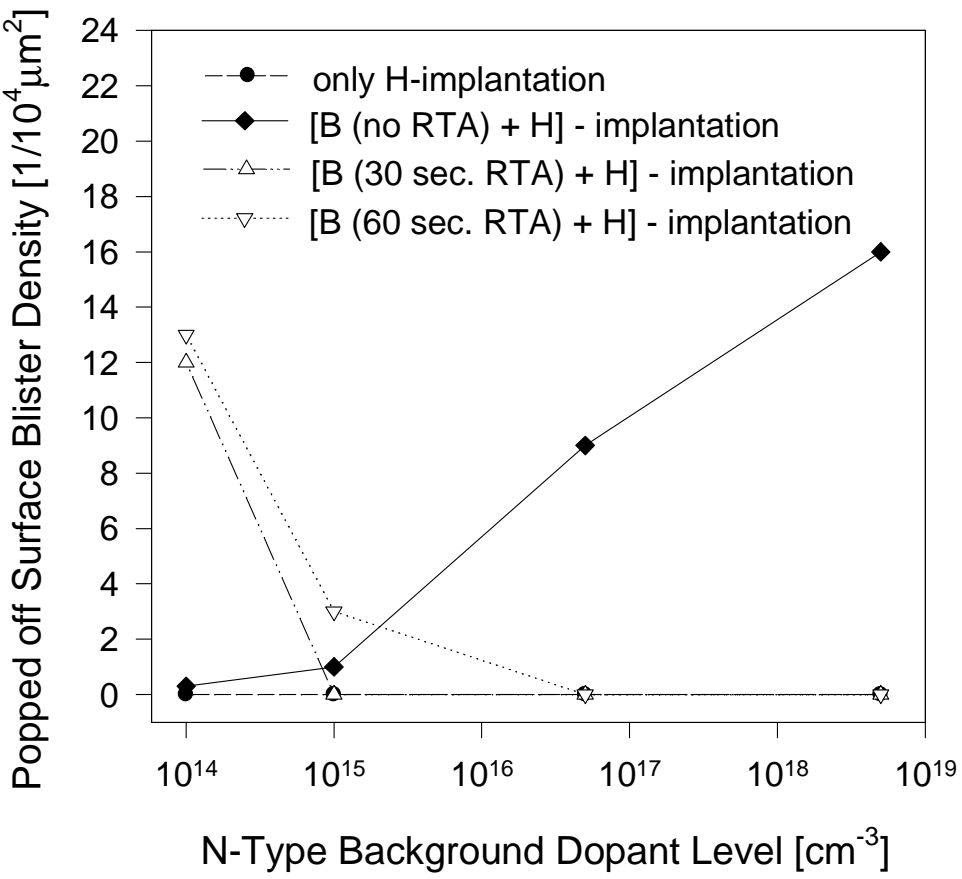


For the boron-activated samples, surface blistering occurs only in the samples with low n-type background doping, and decreases rapidly with increasing n-type dopant level. It is worth noting that the two samples with the lowest n-type dopant of about $10^{14} \mathrm{~cm}^{-3}$ and sample treatment B and C ([B (with RTA) + H]-implantation) have a considerably higher $D_{n}$ compared to all other samples (see figure 7-4). Since these two samples show intense surface blistering after the second anneal, it is reasonable to assume that the conversion of $\mathrm{H}$-complexes into $\mathrm{H}_{2}$ molecules in the as-implanted state is more advanced in these two samples than in all other samples. Sub-surface H-bubbles cause strong lattice distortion within the implantation zone. Therefore, I suggest that the high $\mathrm{D}_{\mathrm{n}}$ values of the two activated boron samples (measured prior to the second anneal) are due to the $\mathrm{H}_{2}$ molecule formations whereas the high dechanneling yield observed only for these two samples prior to the second annealing (see figure 7-3, channel region 250-340) is caused by lattice distortion arising from the bubble nuclei.

Figure 7-14 presents as an example the cross section SEM image of a surface blister in the sample with a n-type dopant level of about $10^{14} \mathrm{~cm}^{-3}$ and sample treatment $\mathrm{C}$ (see Table 7-2). Similar observations on various blisters indicate that the roughness of the blister depth amounts to between 5 and $10 \mathrm{~nm}$.

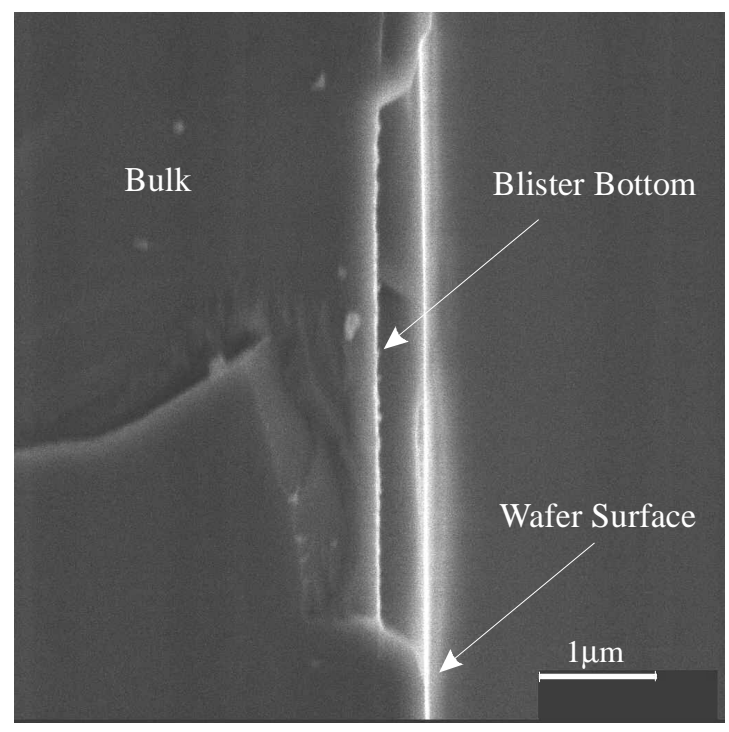

Figure 7-14: Cross section image of a "popped off" surface blister obtained by SEM. The Si sample had a resistivity of $30-50 \Omega \mathrm{cm}$ and was [B (RTA for $60 \mathrm{sec}$. at $\left.900^{\circ} \mathrm{C}\right)+\mathrm{H}$ ] implanted and subsequently annealed at $390^{\circ} \mathrm{C}$ for 10 minutes.

Figure 7-15 shows the average depth of the surface blisters as a function of the n-type background dopant level. Also shown in this figure are the depths for the hydrogen implantation peak $(435 \mathrm{~nm})$, the boron implantation peak $(690 \mathrm{~nm})$ and the hydrogen implantation damage peak $(405 \mathrm{~nm})$. Although the roughness of the depth of the individual blisters is very small, for each sample the depths of various surface blisters 
differ more, as indicated by the error bars. The data indicate that whenever blisters form, they do so in average at the depth region of maximum damage centered at a depth of about $405 \mathrm{~nm}$, or slightly shallower rather than the peak in the H-distribution, centered at a depth of about $435 \mathrm{~nm}$. (TRIM calculations (see figure 7-1) predicted a somewhat deeper H-distribution. But since the stopping powers used for the simulations are inaccurate, the combination of ERD and SIMS measurements provides a more precise assessment of the H-depth distribution.)

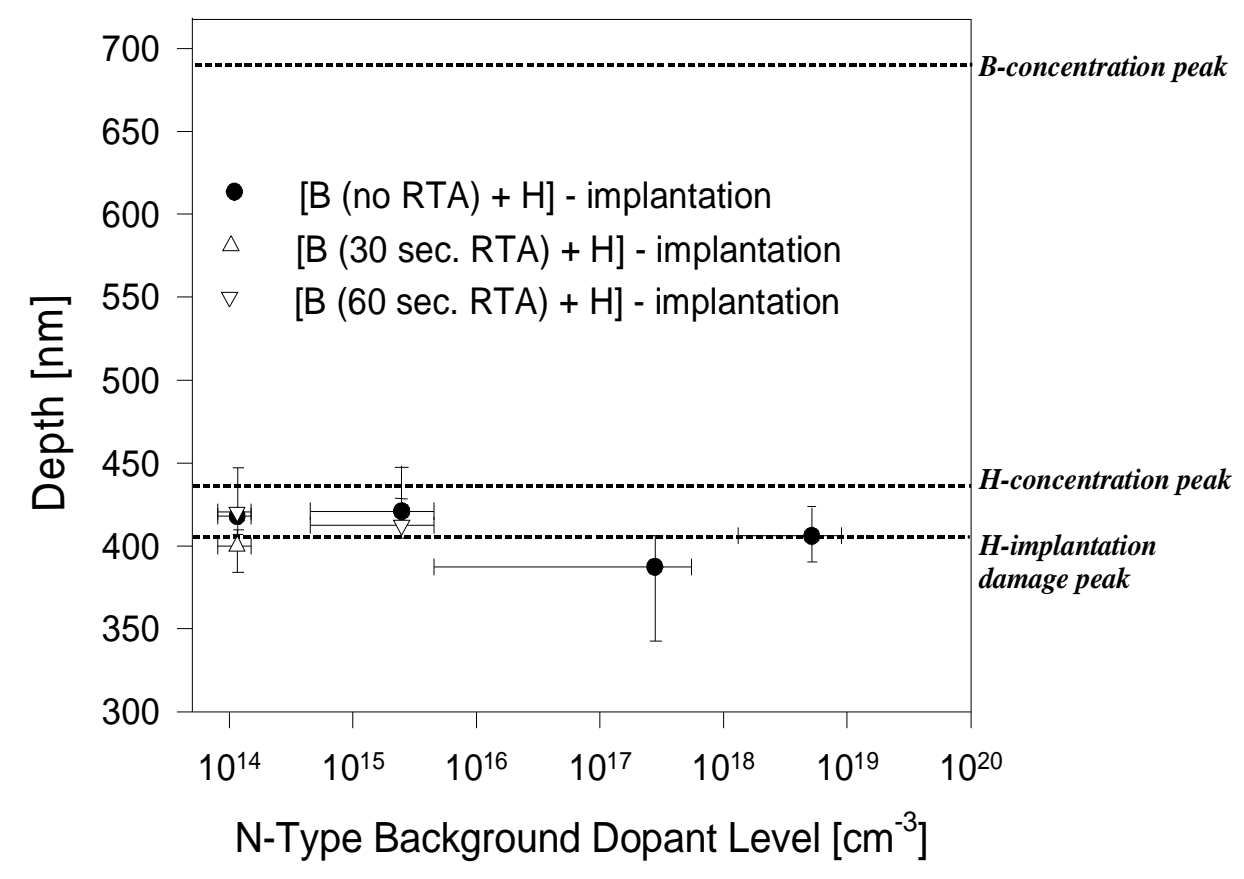

Figure 7-15: Average depth of surface blisters after second annealing versus background dopant level. The depth of seven blisters were measured by SEM for each sample treatment. The bars indicate the deepest and shallowest depth of the blisters for each sample treatment.

The results of the blister depth measurements are consistent with the observations made in chapter 5. Boron activation prior to the hydrogen-implantation does not seem to influence the location of surface blister formation. 


\subsection{The Influence of the Pre-Implanted Boron on the Surface Blister Formation}

The observed blister depths, confined to a narrow range of depth, suggest that the variation in surface blister densities shown in figure 7-13 is not the result of differences in the energy required to induce deformation and fracture of the silicon surface layer located above a H-bubble. In all cases the thickness of the Si over-layer is nearly the same.

Therefore, above results show that the presence of the pre-implanted boron and its electrical state plays an important role in the H-platelet formation and their growth into $\mathrm{H}_{2}$-gas bubbles. This section discusses the results obtained on sample treatments $\mathrm{A}, \mathrm{B}$ and $\mathrm{C}$ (co-implantation without and with boron-activation) (see Table 7-2).

\section{Blister formation in the $B+H$ implanted samples when the boron is not activated}

A comparison between $D_{n}$ (figure 7-4) and the surface blister density (figure 7-13) for the [B (no RTA) + H] - implanted samples ( $\diamond$-data) shows some correlation between these two data. Similar to $D_{n}$, the blister density increases with the n-type background dopant level. This suggests a correlation between blister formation and implantation damage.

Comparing the defect data in figure 7-4, it becomes evident that all samples implanted with hydrogen only (-data) have higher or at least as high values of $D_{n}$ than the $[\mathrm{B}($ no RTA $)+\mathrm{H}]$ - implanted samples $(\diamond$-data). However, this damage increase is not manifested in the surface blistering data shown in figure 7-13. No surface blistering is observed in the hydrogen-only implanted samples despite their larger value of $D_{n}$. This observation indicates that the presence of boron is critical for blister formation and that damage and its enhancing effect on H-platelet formation (see chapter 5-1) alone cannot explain the blistering trends observed.

To assess the role of damage alone, without possible complications from chemical effects, I also studied Si samples self-implanted with $\mathrm{Si}$ ions $(380 \mathrm{keV}$ at a dose of $5 \times 10^{13} \mathrm{~cm}^{-2}$ ). The Si-implantation was done prior to the $\mathrm{H}$-implantation and was not followed by a RTA. Tong et al. [156] have reported that in B-implanted samples the stress induced by the B-implantation is not the cause for the observed enhancement in 
surface blistering. For the present samples, which were implanted with a low Si dose, the anticipated strain is low. [158] Furthermore, the damages caused by $\mathrm{Si}$ and Bimplantation are comparable, as determined by RBS channeling measurements: Figure 7-16 presents the RBS channeling spectra, obtained from the boron implanted and the silicon self implanted samples, respectively. The plot shows almost identical backscattering yields in the two channeling spectra.

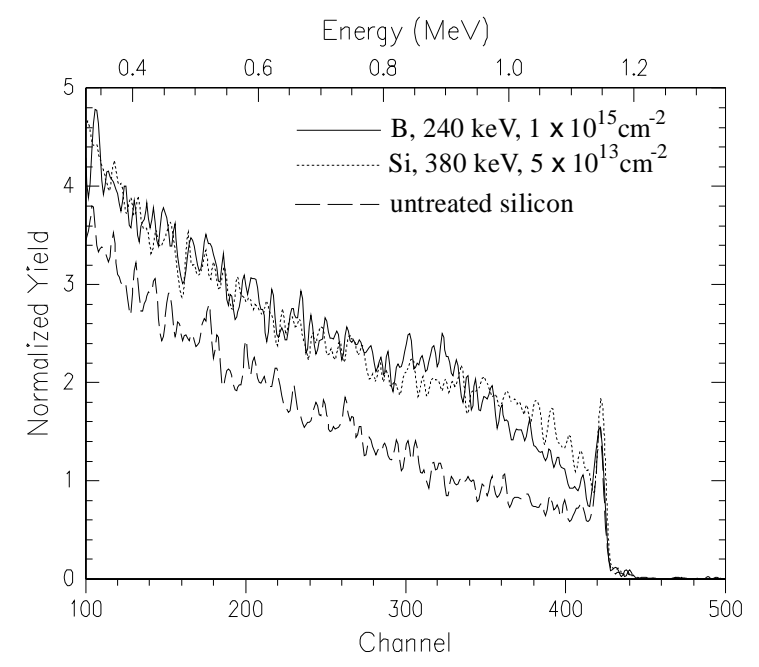

Figure 7-16: $2.0 \mathrm{MeV}$ He RBS-channeling spectra of the $<100>$ n-type silicon sample with a resistivity of $0.005-0.020 \Omega \mathrm{cm}$ after room temperature implantation of $240 \mathrm{keV}$ B-ions to a dose of $1 \times 10^{15} \mathrm{~cm}^{-2}$ and $380 \mathrm{keV}$ Si-ions to a dose of $3 \times 10^{13}$, respectively. For comparison the channeling spectrum of untreated silicon is shown, too.

Because the Si samples self-implanted with $\mathrm{Si}$ and then followed by H-implantation showed no surface blistering after annealing, this result confirms that implantation damage alone is not responsible for surface blistering, as concluded earlier. This experiment underlines the chemical importance of boron in hydrogen blister formation. Previous studies have shown that boron in silicon acts as a multiple trap for hydrogen. [48-50] It is my belief that the boron atoms located within the implanted hydrogen region behave as local traps for atomic hydrogen which then promote the nucleation of hydrogen platelets. Additional atomic hydrogen diffuses to these nuclei during the second anneal. This causes the platelets to grow and evolve into micro cracks, leading finally to surface blistering.

The increase in the surface blister density with increasing n-type background dopant level in the [B (no RTA) + H] - implanted samples (figure 7-13) may be due to a change in the charge state of the implanted hydrogen. Figure 7-17 shows a schematic diagram of the electronic levels for the different states of atomic hydrogen in $\mathrm{Si}$. The donor level of hydrogen atoms located at the bond-centered sites is $E_{D}{ }^{B C}=E_{C}-0.16 \mathrm{eV}$ [159], where $E_{C}$ is the energy at the bottom of the conduction band. In intrinsic $\mathrm{Si}$, the Fermi energy is located at the middle of the energy band gap. Therefore, in intrinsic Si, implanted hydrogen atoms residing at the bond-centered sites are in the $\mathrm{H}^{+}$state. As the n-type dopant level increases and the Fermi level crosses $\mathrm{E}_{\mathrm{D}}{ }^{\mathrm{BC}}$, 
the bond-centered hydrogen atoms are expected to change from $\mathrm{H}^{+}$to $\mathrm{H}^{0}$ states. At the second anneal temperature of $390{ }^{\circ} \mathrm{C}$ this crossover occurs when the n-type dopant level exceeds about $5 \times 10^{18} \mathrm{~cm}^{-3}$. [160]

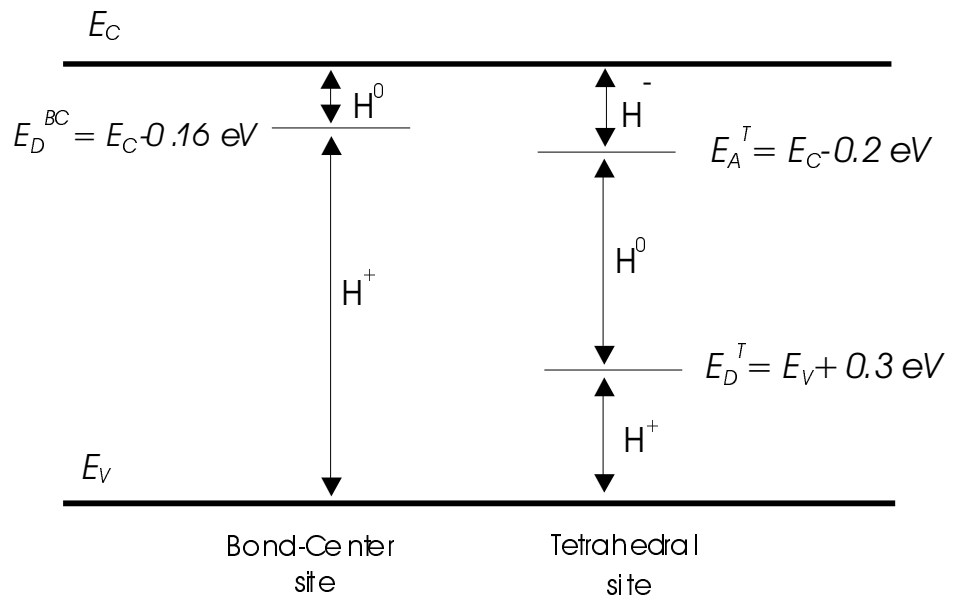

Figure 7-17: Schematic diagram of the electronic levels of a single hydrogen atom in silicon located at the bond-centered and tetrahedral site, respectively (data from Ref. [159]). The arrows indicate the charge state of the hydrogen atoms when the Fermi energy is within that region.

The acceptor level $\mathrm{E}_{\mathrm{A}}{ }^{\mathrm{T}}$ of atomic hydrogen residing at tetrahedral lattice sites is located at $\mathrm{E}_{\mathrm{A}}^{\mathrm{T}}=\mathrm{E}_{\mathrm{C}}-0.2 \mathrm{eV}$. [159] Thus, in intrinsic $\mathrm{Si}$ the implanted hydrogen located at the tetrahedral site is predominantly in the neutral charge state. However, these states are unstable and the hydrogen atoms move to the bond-centered position, where they become positively charged at temperatures $>150 \mathrm{~K}$. [161] With increasing n-type dopant level the Fermi level moves up towards the bottom of the conduction band. When the n-type dopant level reaches about $10^{16} \mathrm{~cm}^{-3}$, the Fermi level crosses the acceptor level $\mathrm{E}_{\mathrm{A}}^{\mathrm{T}}$. Therefore, when the n-type dopant level exceeds about $10^{16} \mathrm{~cm}^{-3}$ (resistivity $<0.5 \Omega \mathrm{cm}$ ) the atomic hydrogen sitting on tetrahedral sites will be predominantly in the negative charge state.

The donor level $\mathrm{E}_{\mathrm{D}}^{\mathrm{T}}$ of atomic hydrogen residing at tetrahedral lattice sites is located at $E_{D}{ }^{T}=E_{V}+0.3 \mathrm{eV}$. [159] For p-type $\mathrm{Si}$, the Fermi energy is low, near the valence band, and the hydrogen atoms in tetrahedral sites are in a $\mathrm{H}^{+}$state.

It is not known how the change in the charge state of the implanted hydrogen influences the hydrogen blister formation. It is known, however, that the diffusion coefficient of hydrogen is larger when the impurity is in the positive charge state than in the negative or neutral charge states. [162] However, hydrogen platelet formation has 
been shown to be more prevalent if the diffusing hydrogen is in the neutral or negative charge state. [162]

Therefore, I assume the large amount of $\mathrm{H}^{-}$to be critical for the surface blister formation observed for the $[\mathrm{B}($ no RTA $)+\mathrm{H}]-$ implanted samples. The increasing amount of hydrogen expected in the neutral charge state (located at the bond centered site) and negative charge state (located at the tetrahedral site) with increasing n-type dopant level is assumed to lead to an intensification of hydrogen platelet formation and finally to the larger surface blister density with increasing n-type background dopant level indicated in figure 7-13.

\section{Blister formation in the $B+H$ implanted samples when the boron is activated}

The mechanism for surface blister formation in $[B+H]$ - implanted samples that were given a RTA treatment prior to the hydrogen-implantation is somewhat different from that responsible for hydrogen blister formation in similar samples with no RTA. For the samples with the two lower n-type dopant levels (ca. $10^{14} \mathrm{~cm}^{-3}$ and $10^{15} \mathrm{~cm}^{-3}$ ) surface blistering was mainly observed when the implanted boron was activated (figure 7-13).

SIMS and ERD measurements showed that the as-implanted hydrogen depth distribution changes with thermal annealing. The H-implant diffuses towards the deeper boron depth distribution. However, the majority of the hydrogen located in the region of high $\mathrm{H}$-implantation damage does not diffuse towards the bulk (see figure 7-10). In addition, surface blistering has been found to occur in the region of maximum hydrogen implantation damage. Thus, the data obtained in this series of experiments suggest that implantation damage acts as local trap sites for the implanted hydrogen and therefore suppresses more intense long-range diffusion of the implanted hydrogen towards the boron. This hypothesis is consistent with previous experiments. [124] Hence, the differences in surface blister formation are assumed to be caused by, among other things, the amount of short range diffusion which is required for the hydrogen atoms located near the blister depth to reach the closest H-bubble precursor.

The as-implanted boron is in a neutral electrical state. The rapid thermal anneal of the B-implanted samples at the temperature of $900{ }^{\circ} \mathrm{C}$ leads to an electrical activation of the boron. The electrical activation yield or the ratio between activated p-type carrier concentration and implanted boron concentration reaches a value of about $25-30 \%$ after 
the RTA. [163] Previous studies have also shown that a significant fraction of the implanted boron is activated during the first few seconds of the RTA and continues to increase with increasing annealing time at the chosen RTA temperature. [163] Therefore, a slightly higher electrical activation yield is expected in the samples rapid thermal annealed for 60 seconds than in those rapid thermal annealed for only 30 seconds.

Because the boron concentration at the peak of the hydrogen distribution is about $2 \times 10^{18} \mathrm{~cm}^{-3}$ (see figure 7-1), the concentration of the electrical activated boron is expected to be about $5 \times 10^{17} \mathrm{~cm}^{-3}$. This is considerable larger than the n-type background dopant levels of the two lower n-type doped samples. Hence, following the RTA, the conductivity of the [B (with RTA) + H] - implanted samples with the two lower n-type background dopant levels (ca. $10^{14} \mathrm{~cm}^{-3}$ and $10^{15} \mathrm{~cm}^{-3}$ ) is p-type within the depth where the hydrogen is located. With increasing n-type background doping the boron concentration cannot cause a change in the conductivity type and the material remains n-type. With less n-type background doping, the concentration of electrically active boron increases which results in a corresponding shift of the Fermi level towards the valence band $\mathrm{E}_{\mathrm{V}}$. For the two lower n-type doped samples, after the boron implantation and activation, the Fermi level crosses the donor level for $\mathrm{H}$ in the bond-centered site $\mathrm{E}_{\mathrm{D}}^{\mathrm{BC}}$ and for $\mathrm{H}$ at the tetrahedral site $\mathrm{E}_{\mathrm{D}}^{\mathrm{T}}$ (see figure 7-17). Therefore, at the assumed p-type dopant level, the implanted hydrogen is expected to be mainly in the positive charge state. $[159,164]$ Hydrogen in the positive charge state has a very high diffusion coefficient [165] and therefore the kinetics of H-bubble nucleation and growth should be enhanced which, in turn, should enhance surface blister formation. In addition the electrons, donated by the $\mathrm{H}$, and the $\mathrm{H}^{+}$, set up an internal electric field. This electric field leads to a further enhancement of the hydrogen diffusion due to the electrical field drift effect on the protons.

The high hydrogen diffusion coefficient in combination with the large amount of boron in the depth range of the $\mathrm{H}$-depth distribution, acting as a multiple trap of hydrogen, is considered to lead to the surface blistering for the [B $(30 \mathrm{sec}$. RTA $)+\mathrm{H}]-$ implanted sample with the lowest n-type background dopant level and for the $[\mathrm{B}(60 \mathrm{sec}$. RTA $)+\mathrm{H}]$ - implanted sample with the two lower n-type background dopant levels. This mechanism of surface blister formation is consistent with the RBS channeling results, showing very large backscattering and dechanneling yields in the [B (with 30/60sec. RTA) + H] - implanted samples with the lowest n-type background dopant level prior to the second anneal (see right part of figure 7-3). The high channeling yields indicate the presence of $\mathrm{H}$-filled platelet or bubble formation directly after hydrogen-implantation. 
The amount of $\mathrm{H}^{+}$and its high diffusion coefficient also explains the observed dependence of the surface blister density as a function of RTA duration. By increasing the RTA duration from 30 to 60 seconds a corresponding increase in the amount of activated boron results, which consequently increases the concentration of $\mathrm{H}^{+}$. The data in figure 7-13 show that the net result is an increase in the surface blister density in the lowest n-type doped sample and the observation of blister formation in the sample with the second lowest n-type dopant level.

To verify the above interpretation, hydrogen was implanted into a $<100>$ p-type silicon wafer (boron doped) with a resistivity of $0.005-0.009 \Omega \mathrm{cm}$. The corresponding activated boron doping level of this wafer is $1.3 \times 10^{18}-9.0 \times 10^{18} \mathrm{~cm}^{-3}$, which is comparable to that obtained in the [B (with RTA) $+\mathrm{H}]$-implanted samples at the depth of the hydrogen distribution. The hydrogen-implantation and second annealing was carried out with the same parameters as above. Plane view SEM imaging of this sample reveals a density of 18 surface blisters $/ 10^{4} \mu \mathrm{m}^{2}$ after the second anneal (see figure 7-18). This value is similar in magnitude to the density obtained in the $[\mathrm{B}(60 \mathrm{sec}$. RTA $)+\mathrm{H}]-$ implanted sample with the lowest n-type background dopant level.

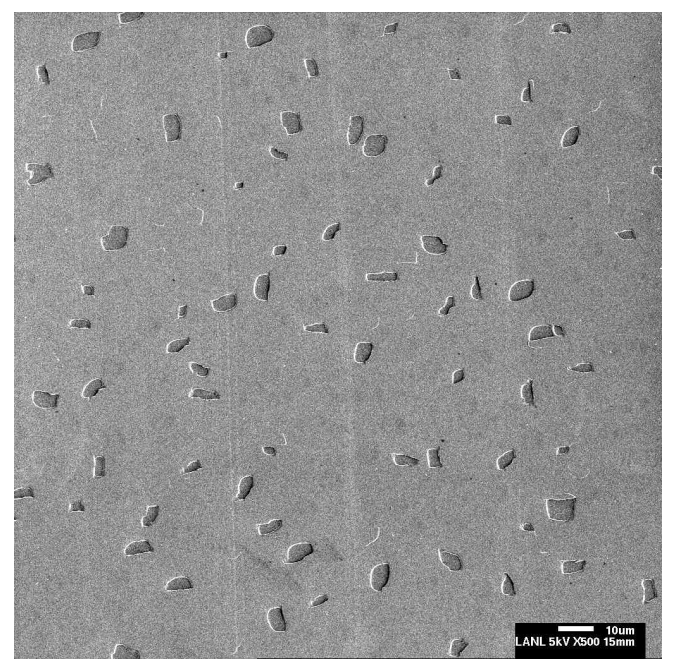

Figure 7-18: Plane view SEM image spectrum of the $40 \mathrm{keV} \mathrm{H}$-ion implanted $<100>$ p-type Si wafer with a electrically active boron dopant concentration of $9.0 \times 10^{18}-1.5 \times 10^{19} \mathrm{~cm}^{-3}$ after annealing at $390{ }^{\circ} \mathrm{C}$ for 10 minutes in vacuum. The $\mathrm{H}$-implantation dose was $5 \times 10^{16} \mathrm{~cm}^{-2}$. The image shows intense surface blistering, although no additional boron was implanted prior to the $\mathrm{H}$-ion implantation.

For the samples with the two higher n-type background dopant levels the amount of incorporated electrical activated boron after the [B (with 30/60sec. RTA) $+\mathrm{H}]$ implantation treatment is not enough to induce a shift of the Fermi level towards the donor levels of the hydrogen, in contrast to the samples with the two lower n-type background dopant levels. Therefore, for the two higher n-doped samples, the [B (with 30/60sec. RTA) + H] - implantation treatments do not result in a high concentration of fast diffusing hydrogen in the positive charge state and as a result surface blisters were not obtained after second annealing. 


\section{The influence of boron at interstitial lattice sites on hydrogen blister formation}

For the two higher n-type doped samples, surface blistering is only observed after the second anneal, when the implanted boron was not activated. The charge state of the implanted hydrogen for these two n-type background dopant levels is expected to be the same for activated and non-activated boron. Hence, at these n-type dopant levels, the main difference between the $[\mathrm{B}($ no RTA $)+\mathrm{H}]$ - implanted samples and the $[\mathrm{B}($ with RTA $)+\mathrm{H}]$ - implanted samples is the recovery of the boron implantation damage and a higher amount of substitutional boron when RTA was carried out. This suggests that boron located at interstitial sites has an enormous influence on hydrogen bubble formation. This hypothesis is confirmed by recent experimental studies on $\mathrm{B}+\mathrm{H}$-implanted p-type silicon samples. At a given annealing duration, a greater reduction is achieved in the annealing temperature required to form surface blisters, when pre-implanted boron is present in the as-implanted state compared to pre-implanted boron electrically activated before the H-ion implantation. [156]

In summary, the study has shown that long-range diffusion of hydrogen-implanted into n-type silicon takes place for an annealing temperature of $390{ }^{\circ} \mathrm{C}$. The presence of a preimplanted boron profile, at depths greater than the implanted hydrogen profile, and the long-range hydrogen diffusion it caused did however not induce an Ion-Cut shift towards larger depths. The long-range diffusion of hydrogen at these temperatures appears to be constrained by the presence of the lattice damage produced during the implantation process. The presence of the pre-implanted boron, either in the as-implanted state or electrically activated by RTA, in n-type doped silicon is necessary for surface blister formation at the given implantation and anneal parameters. Samples that were only implanted with hydrogen did not produce surface blisters. The formation and frequency of surface blisters was found to be a function of the n-type background dopant level at the hydrogen-implantation depth and the damage distribution resulting from the implantation of hydrogen. The data suggest that the local conductivity type of boron implanted n-type silicon influences the Fermi level and the ultimate charge state of free hydrogen, which in turn alters the local hydrogen kinetics, short-range diffusion, H-bubble nucleation, and $\mathrm{H}$-bubble growth. The presence of activated boron renders silicon samples with low ntype doping concentration to be effectively p-type material in the region around the 
hydrogen-implantation profile. Under such conditions free hydrogen will be mainly in the positive charge state, will possess high short-range mobility, and will more easily contribute to the growth of hydrogen bubbles. The most probable charge-state of free hydrogen in highly doped n-type silicon is negative, with the concentration of $\mathrm{H}^{-}$ increasing with increasing n-type doping level. Free boron in n-type silicon effectively traps $\mathrm{H}^{-}$which in turn intensifies blister formation. 


\section{Summary}

The "Ion-Cut", a layer splitting process by $\mathrm{H}$-ion implantation and subsequent annealing is an effective, versatile, and economical method of transferring silicon surface layers, that could be as thin as a few tens of nanometers, from bulk substrates onto a host of other substrates, thus enabling the production of silicon-on-insulator (SOI) materials. Cleavage is induced by the coalescence of the highly pressurized sub-surface $\mathrm{H}_{2}$-gas bubbles, which form upon thermal annealing. While the Ion-Cut phenomenon is widely applied, a fundamental understanding of the basic mechanisms on how the cutting process occurs was still unclear, impeding further optimization of the Ion-Cut process.

This work elucidated the physical mechanisms behind the Ion-Cut process in hydrogen implanted silicon by examining the defect evolution that occurs during hydrogen ion implantation and correlation of the findings with the H-depth concentration profile, the microstructure of the implanted sample, and the position of the Ion-Cut within the implantation zone. The investigation of the cleavage process reveals the cut to be largely controlled by the lattice damage, generated by the hydrogen ion irradiation

process, and its effects on the local stress field and the fracture toughness within the implantation zone rather than by the depth of maximum H-concentration.

Detailed studies on samples, implanted with a moderate H-implantation dose showed the Ion-Cut to occur at the range of maximum ion irradiation induced crystal damage, contrary to the general belief that cleavage occurs at the depth of maximum hydrogen concentration. The results were achieved by a combination of ion beam analyses, secondary ion mass spectroscopy, ex-citu measurements of the stress accumulation in the sample, and electron microcopy. The findings were proposed to be caused by the evolution of the silicon lattice damage, generated by the hydrogen ion irradiation process, its effects on the local stress field which in turn leads to elastic tensile out-of plane strains. The elastic tensile out-of-plane strains were found to have an enhancing effect on the incorporation of hydrogen atoms into bond-centered sites between two silicon lattice atoms, thus forming (100) oriented hydrogen platelets parallel to the surface. These hydrogen platelets constitute the nuclei for subsurface $\mathrm{H}_{2}$-gas bubbles, which are responsible for cleavage to occur.

Above hypotheses were confirmed by studying the changes in the Ion-Cut process with increasing hydrogen implantation dose. The investigation of the cleavage process for different proton implantation doses revealed the cut to take place at two distinct 
depths within the implantation zone, either at the depth of maximum damage or in the end-of-tail region of the somewhat deeper H-concentration depth distribution. This work showed for the first time the role of the hydrogen ion irradiation induced defects on the fracture toughness of $\mathrm{H}$-implanted silicon and how the evolving fracture toughness influences the cleavage location. The correlation of the obtained data led to the conclusion that the shift of the Ion-Cut with increasing $\mathrm{H}$ implantation dose towards larger depths is caused by the changing fracture mechanics within the implantation dose. This fundamental understanding of how the cleavage mechanism evolves in silicon as a function of $\mathrm{H}$-ion dose demonstrated for the first time the critical inter-relationship between fracture, stress, and point and extended defects that is responsible for the Ion-Cut process in silicon.

Detailed information about the formation kinetics of hydrogen related complexes in silicon and their influence on the crystal damage and in turn the hydrogen platelet formation and hydrogen platelet orientation were gained from low dose proton implanted samples, annealed at temperatures ranging form $200{ }^{\circ} \mathrm{C}$ to $530{ }^{\circ} \mathrm{C}$. The analyses showed that upon annealing $\mathrm{H}_{2}$ molecule formation within the silicon lattice (not the $\mathrm{H}_{2}$-gas bubbles) leads to a considerable increase of displaced silicon atoms. These $\mathrm{H}_{2}$ molecules are not residing in H-platelets or bubbles, but their influence on the crystal damage and the stress and the strain field in the sample influences the H-platelet formation, their density, and their depth location. These results were gained by examining the changes in the shape of the damage and hydrogen depth profiles in silicon, caused by annealing at different temperatures. The experiments were performed on two silicon substrates with different conductivity types. P-type silicon samples showed somewhat different kinetics in the hydrogen complex formations than n-type silicon samples. These observed dissimilarities result in different hydrogen platelet density depth profiles at different silicon conductivity types.

CSTIM analyses investigated the damage accumulation caused by the crack propagation through the silicon samples in the splitting step of the Ion-Cut process. Thereby, the splitting process was examined in form of "popped off" discrete silicon areas on non-bonded silicon samples. The exfoliation process was found to induce mechanically damage formation in the silicon sample.

Finally, the influence of boron pre-implantation on the Ion-Cut in hydrogen implanted silicon was investigated. The experiments were performed in n-type silicon with different n-type dopant levels such that the influence, the change of the conductivity type and the variation of the Fermi level have on the Ion-Cut process, could be investigated. The experiments revealed, that both, the atomic interaction between the 
boron implant and the hydrogen implant and the shift of the Fermi level due to the electrical activation of the implanted boron have a tremendous enhancing effect on the agglomeration of hydrogen in $\mathrm{H}_{2}$-gas bubbles which in turn induce ion-cutting.

This research contributes to a better understanding of the Ion-Cut phenomenon and therefore facilitates further optimization of this layer splitting process. 


\section{Bibliography:}

[1] Q.Y. Tong, K. Gutjahr, S. Hopfe, U. Gösele, and T.H. Lee, Appl. Phys. Lett. 70, 1390 (1997)

[2] L.Di Cioccio, Y. Le Tiec, F. Letertre, C. Jaussaud, and M. Bruel, Electron. Lett. 32, 1144 (1996)

[3] Q.Y. Tong, T.H. Lee, W.J. Kim, T.Y. Tan, U. Gösele, H.M. You, W. Yun, and J.K.O. Sin, Feasibility study of VLSI device layer transfer by CMP PETEOS direct bonding, IEEE International SOI Conference, FL, USA, (Piscataway, NJ, USA,1996) pp. 36-37

[4] J.P. Collinge, Silicon-on-Insulator Technology: Materials to VLSI, (Kluwer Academic, Boston, 1991)

[5] A. Antilla, J. Hirvonen and M. Hautala, Radiation Effects Lett. 57, 41 (1980)

[6] M. Bister, J. Hirvonen, J. Räisänen and A. Antilla, Radiation Effects 59, 199 (1981)

[7] M. Bruel, B. Aspar, B. Charlet, C. Maleville, T. Poumeyrol, A. Soubie, A.J. Auberton-Herve, J.M. Lamure, T. Barge, F. Metral, and S. Trucchi, 1995, Proc. IEEE Tucson, Arizona, (Piscataway, NJ, USA, 1995) 178

[8] M. Bruel, Nucl. Instr. Meth., B 108, 313 (1996)

[9] M. Bruel, MRS Bulletin 12, No. 23, December, 35 (1998)

[10] L.B. Freund, Appl. Phys. Lett. 70, 3519 (1997)

[11] C.M. Varma, Appl. Phys. Lett. 71, 3519 (1997)

[12] M.K. Weldon, V.E. Marsico, Y.J. Chabal, A. Agarwal, D.J. Eaglesham, J. Sapjenta, W.L. Brown, D.C. Jacobson, Y. Caudano, S.B. Christman, and E.E. Chaban, Electrochem. Soc. Proc. 97, No.36,229 (1998)

[13] J.I. Pankove and N.M. Johnson, eds., Hydrogen in Semiconductors, (Academic Press, Boston, 1991)

[14] C. Maleville, B. Aspar, T. Poumeyrol, H .Moriceau, M. Bruel, A.J. AubertonHerve, and T. Barge, Mat. Sci. and Eng. B 46,14 (1997)

[15] R. Stengl, T. Tan, and U. Gösele, Jpn. J. Appl. Phys. 28, 1735 (1989)

[16] Y. Kim, H.Z. Massoud, U.M. Gösele, and R.B. Fair, Electrochem. Soc. Proc. 91-94, $304(1991)$

[17] A. Van Wieringen, and N. Warmoltz, Physica 22, 849 (1956)

[18] T. Ichimiya, and A. Furuichi, Int. J. Appl Rad. Isot. 19,573 (1968) 
[19] Y.A. Ossipyan, A.M. Rtischer, E.A. Shteinman, E.B. Yakinov, and N.A. Yarykin, Sov. Phsy. JETP 55, 294 (1982)

[20] N.M Johnson, D.K. Biegelsen, and M.D. Moyer, Appl. Phys. Lett. 40, 882 (1982)

[21] S.J. Pearton and A.J. Tavendale, Phys. Rev. B 26, 7105 (1982)

[22] M.W. Horn, J.M. Heddelson, and S.J. Fonash, Appl. Phys. Lett. 51, 490 (1987)

[23] J.T. Borenstein, D. Angell, and J.W. Corbett, Mat. Res. Symp. Proc. 138, 209 (1989)

[24] A.J. Tavendale, A.A. Williams, and S.J. Pearton, Mat. Res. Soc. Symp. Proc. 104, 285 (1988)

[25] T Zundel, A. Mesli, J.C. Muller, and P. Stiffert, Appl. Phys. A 48, 31 (1989)

[26] C.P. Herrero, M. Stutzmann, A. Breitschwerdt, and P.V. Santos, Phys. Rev. B 41, 1054 (1990)

[27] P.E. Blöchl, C.G. Van de Walle, and S.T. Pantelides, Phys. Rev. Lett. 64, 1401 (1990)

[28] W.B. Jackson, Phil. Trans. Roy. Soc. Lond. A 350, 237 (1995)

[29] B.L. Sopori, X. Deng, J.P. Benner, A. Rohatgi, P. Sana, S.K. Estreicher, Y.K. Park, and M.A. Roberson, Solar Energy Mat. and Solar Cells 41/42. 159 (1996),

[30] K.M. Forsythe and N. Makri, J. Chem. Phys. 108, 6819 (1998)

[31] C.G. Van de Walle, P.J.H. Denteneer, Y. Bar-Yam, and S.T. Pantelides, Phys. Rev. B 39, 10791 (1989)

[32] C.G. Van de Walle, Phys. Rev. B 49, 49 (1994)

[33] S.K. Estreicher, J.L. Hastings, and P.A. Fedders, Mat. Sci. and Eng. B 58, 31 (1999)

[34] H.J. Stein, S.M. Myers, and D.M. Follstaedt, J. Appl. Phys. 73, 2755 (1993)

[35] M.K. Weldon, V.E. Marsico, Y.J. Chabal, A. Agarwal, D.J. Eaglesham, J. Sapjeta, W.L. Brown, D.C. Jacobson, Y. Caudano, S.B. Christman, and E.E. Chaban, J. Vac. Sci. Technol. B 15, 1065 (1997)

[36] Y.L. Chabal, M.K. Weldon, Y. Caudano, B.B. Stefanov, and K. Raghavachari, Physica B 273-274, 152 (1999)

[37] B. Nielsen, in: Defects in Semiconductors 17, Materials Science Forum, H Heinrich and W. Jantsch, eds., (Trans Tech, Switzerland, 1993), pp. 143-147

[38] J.D. Holbech, B. Nielsen, R. Jones, P. Sitch, and S. Öberg, Phys. Rev. Lett. 71, p. 875 (1993) 
[39] B.B. Nielsen, J.D. Holbech, R. Jones, P. Sitch, and S. Öberg, Mat. Sci. Forum 143147, 845 (1994)

[40] N.M Johnson, F.A. Ponce, R.A. Street, and R.J. Nemanich, Phys. Rev. B 35, 4166 (1987)

[41] N.H. Nickel, G.B. Anderson, N.M. Johnson, and J. Walker, Phys. Rev. B 62 , 8012 (2000)

[42] G.F. Cerofolini, L. Meda, R. Balboni, F. Corni, S. Frabboni, G. Ottaviani, R. Tonini, M. Anderle, and R. Canteri, Phys. Rev. B 46, 2061 (1992)

[43] A.W.R. Leitch, J. Weber, and V. Alex, Mat. Sci. and Eng. B 58,6 (1999)

[44] C.T. Sah, J.Y.C. Sun, and J.J. Tzou, Appl. Phys. Lett 43,204 (1983)

[45] J.I. Pankove, D.E. Carlson, J.E. Berkeyheiser, and R.O. Wance, Phys. Rev. Lett. 51, 2224 (1983)

[46] C.T. Sah, J.Y.C. Sun, and J.J. Tzou, J.Appl. Phys. 55,1525 (1984)

[47] J.I. Pankove, R.O. Wance, and J.E. Berkeyheiser, Appl. Phys. Lett 45,1100 (1984)

[48] A.D. Marwick, G.S. Oehrlein, and M. Wittmer, Appl. Phys. Lett. 59, 198 (1991)

[49] J.T. Borenstein, J.W. Corbett, and S.J. Pearton, J. Appl. Phys 73, 2751 (1993)

[50] L. Korpas, J.W. Corbett, and S.K. Estreicher, Phys. Rev. B 46, 12365 (1992)

[51] N.M Johnson, C. Herring, and D.J. Chadi, Phys. Rev. Lett. 56, 769 (1986)

[52] K. Bergman, M. Stavola, S.J. Pearton, and J. Lopata, Phys. Rev. B 37, 2770 (1987)

[53] J. Zhu, N.M. Johnson, and C. Herring, Phys. Rev. B 41, 12354 (1990)

[54] N. Bohr, Phil. Mag. 25,10 (1913)

[55] N. Bohr, Phil. Mag. 30,581 (1915)

[56] N. Bohr, Mat. Fys. Medd. Dan. Vid. Selsk. 18, No. 8 (1948)

[57] H.A. Bethe, Ann. Physik 5, 325 (1930)

[58] H.A. Bethe, Z. f. Physik 76, 293 (1932)

[59] H.A. Bethe and W. Heitler, Proc. Roy. Soc. A 146, 83 (1934)

[60] F. Bloch, Ann. Physik 16, 287 (1933)

[61] F. Bloch, Z. f. Physik 81, 363 (1933)

[62] O.B. Firsov, Zh. Eksp. Teor. Fiz. 33,696 (1957) 
[63] O.B. Firsov, JETP 7, 308(1958)

[64] J. Lindhard, M. Scharff, and H.E. Schiott, Mat. Fys. Medd. Dan. Vid. Selsk. 33, No. $14(1963)$

[65] J. Lindhard and A. Winter, Mat. Fys. Medd. Dan. Vid. Selsk. 34, No. 4 (1964)

[66] J .Lindhard, V. Nielsen, and M Scharff, Mat. Fys. Medd. Dan. Vid. Selsk. 36, No. $10(1968)$

[67] J .Lindhard, V. Nielsen, M Scharff, and P.V. Thomsen, Mat. Fys. Medd. Dan. Vid. Selsk. 33, No. 10 (1968)

[68] J.W. Mayer, L. Errikson, and J.A. Davies, Ion Implantation in Semiconductors, (Academic Press, New York, 1970)

[69] J.F. Ziegler, ed., Ion Implantation Science and Technology, (Academic Press, New York 1988)

[70] M. Nastasi, J.W. Mayer, and J.K. Hirvonen, Ion-Solid Interaction, Fundamentals and applications, (Cambridge University Press, Cambridge, Great Britain, 1996)

[71] J.P. Biersack, in: Ion Beam Modification in Insulators, P. Mazzoldi and G.W. Arnold, eds., (Elsevier, Amsterdam, 1987), chapt. 1

[72] J.F. Ziegler, Stopping Power and Ranges of Ions In Matter, Vols. 2-6, (Pergamon Press, New York, 1977-1980)

[73] P.D. Townsend, J.C. Kelly, and N.E.W. Hartley, Ion Implantation, Sputtering, and their Application, ( Academic Press, London, 1976)

[74] J. Lindhard and M. Scharff, Phys. Rev. 124, 128 (1961)

[75] J.F. Ziegler, J.B. Biersack and U. Littmark, The Stopping and Range of Ions in Solids, Vol. 1, (Pergamon, New York, 1985)

[76] Model 200-DF4/DF5 Production Ion implanter Operator's Manual, M-F4784001, Varian/Extrion Division, Gloucester, Massachusetts, 1980

[77] J.A. Leavitt, L.C. McIntyre, Jr. and M.R. Weller, in: Handbook of Modern Ion Beam Analysis, J.R. Tesmer, M. Nastasi, eds., (Materials Research Society, Pittsburgh, PA, 1995), p. 42

[78] H.H. Andersen and J.F. Ziegler, Hydrogen Stopping Powers and Ranges in All Elements, (Pergamon Press, New York, 1977)

[79] J.F. Janni, Atomic Data and Nuclear Data Tables 27, 147 (1982)

[80] F. Hubert, R. Bimbot, and H Gauvin, Atomic Data and Nuclear Data Tables 46, 1 (1990) 
[81] G. Konac, S. Kalbitzer, Ch. Klatt, D. Niemann, and R. Stoll, Nucl. Instr. and Meth. B 136-138, 159 (1998)

[82] D. Niemann, G. Konac, S. Kalbitzer, Nucl. Instr. and Meth. B 118,11 (1996),

[83] D.C. Santry and R.D. Werner, Nucl Instr. Meth. B 178, 149 (1991)

[84] L.R. Doolittle, Nucl. Instr. Meth. B 9, 344 (1985)

[85] R.D. Verda, J.R. Tesmer, C.J. Maggiore, and M. Nastasi, Nucl Instr. and Meth. B 183, 391 (2001)

[86] R.D. Verda, C.J. Maggiore, J.R. Tesmer, A. Misra, T. Höchbauer, M. Nastasi, and R.W. Bower, Nucl Instr. and Meth. B 183,401 (2001)

[87] J. R. Tesmer, in: Handbook of Modern Ion Beam Materials Analysis, J.R. Tesmer, M. Nastasi, eds., (Materials Research Society, Pittsburgh, PA, 1995), p. 687.

[88] W.N. Lennard, G.R. Massoumi, T.W. Gibson, and I.V. Mitchell, Nucl. Instr. and Meth. B 152, 370 (1999)

[89] J. Tirira, Y. Serruys and P. Trocellier, Forward Recoil Spectrometry, Application to Hydrogen Determination in Solids, (Plenum Press, New York, 1996), p. 57

[90] P. Bauer, Nucl. Instr. and Meth. B, 45, 673 (1990)

[91] T. Kaneko, Atomic Data and Nuclear Data Table 53, 271 (1993)

[92] J. Tirira, Y. Serruys, and P. Trocellier, Forward Recoil Spectrometry, (Plenum, New York, NY, 1996), pp. 265-271.

[93] E. Szilagyi, Nucl. Instr. and Meth. B, 161-163, 37 (2000)

[94] R.D. Verda, J.R. Tesmer, and M. Natasi, Nucl. Instr. and Meth. B, to be published

[95] E. Rauhala, in: Handbook of Modern Ion Beam Materials Analysis, J.R. Tesmer, M. Nastasi, eds., (Materials Research Society, Pittsburgh, PA, 1995), p.15

[96] A. Benninghoven, F.G. Rüdenauer, and H.W. Werner, Secondary Ion Mass Spectrometry. Basic Concepts, Instrumentational Aspects and Trends, (John Wiley and Sons, 1987)

[97] R.G. Wilson, F.A. Stevie, and C.W. Magee, Secondary Ion Mass Spectrometry: A Practical Handbook for Depth Profiling and Bulk Impurity Analysis, (John Wiley and Sons, 1989)

[98] J.C. Vickerman, A. Brown, and N.M. Reed, Secondary Ion Mass Spectrometry: Principles and Applications, (Oxford University Press, New York, 1989)

[99] W.K. Chu, J.W. Mayer, and M.-A. Nicolet, Backscattering Spectrometry, (Academic Press, New York, 1978), p. 224 
[100] W.K. Chu, J.W. Mayer, and M.-A. Nicolet, Backscattering Spectrometry, (Academic Press, New York, 1978), p. 231

[101] L.C. Feldman, J.W. Mayer, and S.T. Picraux, Materials Analysis by Ion Channeling, (Academic Press, New York, 1982), General Review

[102] G.F. Cerofolini, F. Corni, G. Ottaviani, and R. Tonini, Nucl. Instr. and Meth. B 71, 441 (1992)

[103] H.S. Jin and W.M. Gibson, Nucl. Instr. and Meth. B 13, 76 (1986)

[104] G. de M. Azevedo, M. Behar, J.F. Dias, P.L. Grande, J.H.R. dos Santos, R. Stoll, Chr. Klatt, and S. Kalbitzer, Nucl. Instr. and Meth. B 136-138, 132 (1998)

[105] M.B.H. Breese, D.N. Jamieson, and P.J.C. King, Materials Analysis using a Nuclear Microprobe, (Wiley, New York, 1996)

[106] P.C.J. King, M.B.H. Breese, P.J.M Smulders, P.R. Wilshaw, and G.W. Grime, Nucl. Instr. and Meth. B 118, 426 (1996)

[107] M.H. Loretto, Electron beam analysis of Materials, (Chapman and Hall, London, New York, 1984), chapt. 3

[108] J.I. Goldstein, D.E. Newbury, P. Echlin, D.C. Joy, C. Fiori and E. Lifshin, Scanning Electron Microscopy and X-Ray Microanalysis, (Plenum Press, New York, London, 1981), chapt. 4.2.5

[109] J. Fu, T.V. Vorburger and D. Ballard, Proc. SPIE 2725, 608 (1996)

[110] P.A. Flinn, D.S. Gardner, and W.D. Nix, IEEE Transaction on Electron Devices, ED-34, No. 3,689 (1987)

[111] G.G. Stoney, Proc. Roy. Soc. (London) A82, 172 (1909)

[112] C.A Volkert, 'Wafer Curvature Apparatus', unpublished

[113] M.J. Lobolda and G.A. Toskey, Solid State Technology 41, no. 5, 99 (1998)

[114] T. Höchbauer, M. Nastasi, and J. W. Mayer, Appl. Phys. Lett. 75, 3938 (1999)

[115] S. Roorda, J.M. Poate, D.C. Jacobson, D.J. Eaglesham, B.S. Dennis, S. Dierker, W.C. Sinke, and F. Saepen, Solid State Commun. 75, 197 (1990)

[116] K.B. Winterbon, P. Sigmund, and J.B. Sanders, Mat. Fys. Medd. Dan. Vid. Selsk. 37, no. 14 (1970)

[117] M.F. Ashby and L.M. Brown, Phil. Mag. 8, 1083 (1963)

[118] M. Nastasi, P. Kodali, K.C. Walter, J.D. Embury, R. Ray, Y. Nakamura, J. Mater Res. 4, No. 5, 2173 (1999)

[119] C. S. Nichols, D. R. Clarke, C. G. Van de Walle, Phys. Rev. Lett. 63, 1090 (1989) 
[120] C. G. Van de Walle, in Hydrogen in Semiconductors, J.I. Pankove and N. M. Johnson, eds. (Boston: Academic Press, 1991), chapter 16, pp. 585-622

[121] J. Grisola, G. Ben Assayag, A. Claverie, B. Aspar, C. Lagahe, and L. Laanab, Appl. Phys. Lett. 76, 852 (2000)

[122] Q. Y. Tong, T. H. Lee, L .J. Juang, Y. L. Chao, and U. Gösele, Electronic Letters 34, no. 4, 407 (1998)

[123] A.D. Marwick, in: Hydrogen in Semiconductors, J.I. Pankove and N. M. Johnson, eds., (Boston: Academic Press, 1991), chapter 9, pp. 200-225

[124] D. Fink, J. Krauser, D. Nagengast, T. Almeida Murphy, J. Erxmeier, L. Palmetshofer, D. Bräunig, and A. Weidinger, Appl. Phys. A 61, 381 (1995)

[125] P. Deak, L.C. Snyder, and J.W. Corbett, Phys. Rev. B 37, 6887 (1988)

[126] K.J. Chang and D.J. Chadi, Phys. Rev. Lett. 60, 1422 (1988)

[127] H. Heinrich and W. Jantsch (eds.), Defects in Semiconductors 17, Materials Science Forum, (Trans. Tech. Switzerland,1993), p. 143

[128] C.A. Volkert, J. Appl. Phys. 70, 3521 (1991)

[129] A. Misra, S. Fayeulle, H. Kung, T.E. Mitchell, and M. Nastasi, Nucl. Instr. Meth. B 148, 211 (1999)

[130] H. Zillgen and P. Ehrhard, Nucl. Instr. Meth. B 127/128, 27 (1997)

[131] R.S. Averback and M. Ghaly, Nucl. Instr. Meth. B 127/128, 1 (1997)

[132] K.-N. Tu, J.W. Mayer, and L.C. Feldman, Electronic Thin Film Science, (MacMillan Publishing Company, New York, 1992), chapter 4

[133] C.G. Van de Walle, and N.H. Nickel, Phys. Rev. B 51, 2636 (1995)

[134] V. D. Tkachev, G. Holzer, A. R. Chelyadinskii, Phys. Stat. Sol. (a) 85, k43 (1984)

[135] B. M. Paine, N.N. Hurvitz, and V. S. Sperious, J. Appl. Phys. 61, 1335 (1986)

[136] G.F. Cerofolini, R. Balboni, D. Bisero, F. Corni, S. Frabboni, G. Ottaviani, R. Tonini, R.S. Brusa, A. Zecca, M. Ceschini, G. Giebel, and L. Pavesi, Phys. Status Solidi A 150, 539 (1995)

[137] H. Zillgen and P. Ehrhart, Materials Science Forum 258-263, 503 (1997)

[138] M .Tang, L. Colombo, J. Zhu and T. Diaz de la Rubia, Phys. Rev. B 55, 14279 (1997)

[139] S. Mottet and A. Roizés, in Defects and Radiation Effects in Semiconductors 1978, IOP Conference Proceedings 46, ed. J. H. Albany, (Institute of Physics, Bristol, 1979) 
[140] G. D. Watkins, Materials Science in Semiconductors Proc. 3, 227 (2000)

[141] S. K. Estreicher, Phys. Stat. Sol B 217, 513 (2000)

[142] R.L. Meek, W.M. Gibson, and J.P.F. Scellschop, Radiat. Eff. 11, 139 (1971)

[143] E.P. Eernisse, Appl. Phys. Lett. 18, 581 (1971)

[144] A. B. Lidiard and R. Perrin, Phil. Mag. 14, 49 (1973)

[145] B.M. Paine and V. S. Sperious, J. Appl. Phys. 62, 1704 (1987)

[146] A. L. Bement, Jr., R. G. Hoagland, F. A. Smidt, Jr., in Fracture: An Advance Treatise, Volume III, H. Liebowitz, ed. (Academic Press, New York, 1971) p 536

[147] G. Swadener, unpublished results

[148] D. Broek, Elementary Engineering Fracture Mechanics, (Martinus Nijhoff Publishers, Dordrecht, 1987), chapt. 1

[149] J.W. Mayer, L. Eriksson, S.T. Picraux, and J.A. Davis, Can. J. Phys. 46, 663 (1968)

[150] S.S. Lau, S. Matteson, J.W. Mayer, P. Revesz, J. Gyulai, J. Roth, T.W. Sigmon, and T. Cass, Appl. Phys. Lett. 34, 76 (1979)

[151] K. Henttinen, I. Suni, and S. S. Lau, Appl. Phys. Lett. 76, 2370 (2000)

[152] D. Bisero, F. Corni, S. Frabboni, R. Tonini, G. Ottaviani, and R. Balboni, J. Appl. Phys. 83, 4106 (1998)

[153] S.U. Campisano, S. Coffa, V. Raineri, F. Priolo, and E. Rimini, Nucl. Instr. Meth. $\mathrm{B}, \mathbf{8 0 / 8 1}, 514$ (1993)

[154] A.G. Ulyashin, A.I. Ivanov, I.A. Khorunzhii, R. Job, W.R. Fahrner, F.F. Komarov, and A.C. Kamyshan, Mat. Sci. Eng. B 58, 91 (1999)

[155] Y. Zheng, S.S. Lau, T. Höchbauer, A. Misra, R. Verda, X,-M He, M. Nastasi, and J.W. Mayer, J. Appl. Phys. 88, 2972 (2001)

[156] Q.-Y. Tong, R. Scholz, U. Gösele, T.-H. Lee, L.-J. Huang, Y.-L. Chao, and T.Y. Tan, Appl. Phys. Lett. 72, 49 (1998)

[157] A. Battaglia, F. Priolo, and E. Rimini, Appl. Surf. Sci 56, 577 (1992)

[158] G. Bai and M.A. Nicolet, J. Appl. Phys. 70, 649 (1991)

[159] S. Z. Tokmoldin and B.N. Mukashev, J. Phys. Condens. Matter 3, 9363, (1991)

[160] J.W. Mayer and S.S. Lau, Electronic Material Science: For Integrated Circuits in Si and GaAs, (Macmillan, New York, 1990) chapt.3 
[161] B. Holm, K.B. Nielsen, and B.B. Nielsen, Phys. Rev. Lett. 66, 2360 (1991)

[162] S.J. Pearton, Mater. Sci. Eng. B 23, 130 (1994)

[163] J.P. Souza and H. Boudinov, Appl. Phys. Lett. 66, 3173 (1995)

[164] S.J. Pearton, W. Corbett, and M. Stavola, Hydrogen in Crystalline Semiconductors, Springer series in Materials Science Vol. 16 (Springer, New York, 1992), chapt. 9

[165] C. Herring and N.M. Johnson, in: Hydrogen in Semicondutors, J.I. Pankove and N.M. Johnson, eds. (Academic Press, Boston, 1991), chapt. 10 



\section{Publications:}

\section{List of published journal articles on results from this work:}

[1] The use of ion channeling and elastic recoil detection in determining the mechanism of cleavage in the ion-cut process

Höchbauer T., Nastasi M., Verda R. D., Misra A., Henttinen K., Suni I., Lau S. S., Mayer J. W.,

Nuclear Instruments and Methods in Physics Research B, accepted

[2] Geometric Considerations Relevant to Hydrogen Depth Profiling by Reflection Elastic Recoil Detection Analysis

Verda R.D., Maggiore C.J., Tesmer J.R., Misra A., Höchbauer T., Nastasi M., Bower R.W.,

Nuclear Instruments and Methods in Physics Research B, October 2001, vol. 183, no. 3-4, p. 401-412

[3] Investigation of the cut location in hydrogen implantation induced silicon surface layer exfoliation

Höchbauer T., Misra A., Nastasi M., Mayer J. W., Journal of Applied Physics, June 2001, vol. 89,no. 11, p. 5980-5990

[4] The Influence of Ion Implantation Damage on Hydrogen induced Ion-Cut Höchbauer T., Misra A., Verda R., Zheng Y., Lau S. S., Mayer J. W., Nastasi M. Nuclear Instruments and Methods in Physics Research B, April 2001, vol. 175-177, p.169-175

[5] Hydrogen-implantation induced silicon surface layer exfoliation Höchbauer T., Misra A., Verda R., Nastasi M., Mayer J. W., Zheng Y., Lau S. S. Philosophical Magazine B, November 2000, vol. 80, no. 11, p. 1921-1931

[6] Study of the crystalline quality of exfoliated surfaces in hydrogen-implanted silicon. Breese, M.B.H.; Alvesn, L.C.; Hoechbauer, T.; Nastasi, M.

Applied Physics Letters; 10 July 2000; vol.77, no.2, p.2682-70

[7] Hydrogen blister depth in boron and hydrogen co-implanted n-type silicon.

Höchbauer T.; Nastasi M.; Mayer J.W.

Applied Physics Letters; 20 Dec. 1999; vol.75, no.25, p.3938-3940

[8] The influence of boron ion implantation on hydrogen blister formation in n-type silicon.

Höchbauer T., Walter K. C., Schwarz R.B., Nastasi M., Bower, R.W., Ensinger W.

Journal of Applied Physics; 15 Oct. 1999; vol.86, no.8, p.4176-4183 


\section{Conference Proceedings:}

[9] Influence of damage and dopant on the blister formation in hydrogen implanted silicon Höchbauer T.; Nastasi M., Lau S. S., Zheng Y., Mayer J. W. Materials Research Society Symposium - Proceedings; 1999; v.568, p.109-114

\section{Oral Presentations Given at International Conferences}

[10] 15th International Conference on Ion Beam Analysis, Past, Present and Future, Cairns, Australia, July 15-20, 2001, Oral Presentation: The use of ion channeling and elastic recoil detection in determining the mechanism of cleavage in the ion-cut process.

[11] 16th International conference on the Application of Accelerators in Research and Industry, Denton, Texas, USA, 1-5 November 2000: Invited Talk: The Influence of Damage on Ion-Cut Si

[12] 12th International Conference on Ion Beam Modification of Materials, Canela Rio Grande do Sul, Brazil, 3-8 September, 2000: Oral Presentation: The Influence of Ion Implantation Damage on Hydrogen induced Ion-Cut

[13] 1999 MRS Spring Meeting, San Francisco, USA, April 5-9, 1999. Oral Presentation: The influence of damage and dopant on the blister formation in hydrogen implanted silicon. 


\section{Acknowledgements:}

This work was primarily conducted at Los Alamos National Laboratory (LANL) and supported by the US Department of Energy, Office of Basic Energy Sciences, Division of Material Sciences. I would especially like to thank my research advisor, Mike Nastasi, of LANL. Thank-you for the great interactions regarding the materials science, and for sharing your wealth of knowledge with me. Thank-you also for being understanding during times of personal strife, and at other times by being demanding of me when I needed to be challenged. Without your help this project would not have been possible.

I would also like to thank Wolfgang Ensinger, my PhD advisor at the PhilippsUniversity of Marburg for giving me the opportunity to obtain my doctorate degree. Thank-you for all of your help in taking care of countless things in Germany while I was so far from home.

Thanks to S.S. Lau from the University of California San Diego and Jim Mayer from the Arizona State University, who were collaborators on this project. I benefited immensely from all of our interactions, both personal and those related to the science. Special thanks to Jim for making it possible for me to do the SIMS measurements at the Arizona State University. Your help is greatly appreciated.

Thanks also to my colleagues Ray Verda and Amit Misra, who I shared many fruitful discussions with that no doubt added to the integrity of my work. Ray did a lot of great work on the IBA analysis, and Amit performed the TEM imaging for me. Thanks to both of you. My gratitude goes also to my colleague Ricardo Schwarz for his input concerning the work on $\mathrm{B}+\mathrm{H}$ co-implantation.

My thanks goes also to Yan Zheng from the University of California, San Diego, and to the collaborators on this project, Kimmo Henttinen and Ilkka Suni, for performing wafer bonding and also for many profitable discussions.

Special thanks to the crew at the Ion Beam Materials Lab: Joe Tesmer, Chris Wetteland, Carl Maggiore, Caleb Evans, and Mark Hollander. Thank-you for your enormous support throughout the whole duration of this work. You were always willing to help me in any way you could regardless of your full schedules or the short notice that I gave you when I needed your help. Your technical knowledge of the Implanter ensured that any problems with the equipment would be fixed within minutes instead of weeks, saving me much time and energy. 
Thanks to all those you provided technical support to me throughout the course of my work. Jim Smith and Rob Dickerson of LANL both provided great technical support regarding the use of the SEM and TEM equipment. Rick Hervig from the Arizona State University provided support with the SIMS measurements. Your assistance was extremely helpful to me. Thanks also to Randy Groves for the assistance regarding the AFM measurements.

Thanks to Mark Breese from the University from Surrey in the UK and L. C. Alves from ITN in Sacavĕm, Protugal for performing the CSTIM measurements and for the fruitful discussion providing insight into this analysis method.

I greatly appreciate the support of many people in my Materials Science \& Technology -8 group. I would like to thank the MST-8 group leaders, Mike Stevens and Kathi Alexander, for being so supportive of my work. In particular I would like to thank the administrative assistants in our group, Dorothy Lucero, Lindy Salazar, Dusty Deaguero, and Geraldine Vigil for being so kind and helpful to me, no matter what I needed, from getting paperwork done to helping me interact with the INS when necessary. You have all been a great help to me.

I greatly appreciated the support I received from all my friends throughout this work. Special thanks to Zoran, Susanne, Wendy, Kim, and Jeff. Thank-you Kim that I always could count on you when things got crazy. Thanks to Cindy and Laura for your help during the last phase of this work. A very special thanks goes to Dee Dee! Thanks you so much for your patience, the emotional support, and the organizational help in finishing this work.

Last but not least, I want to thank my parents and siblings for their emotional support throughout these years. They kept me grounded. 